University of Redlands

\title{
Integrating an Agent-based Model into a Web-Enabled Annual Brome Land Management System
}

A Major Individual Project submitted in partial satisfaction of the requirements for the degree of Master of Science in Geographic Information Systems

by

Sean Phillip Murphy

Mark Kumler, Ph.D., Chair

R. Maxwell Baber, Ph.D.

Philip Munger, Ph.D.

July 2008 
Integrating an Agent-based Model into a Web-Enabled Annual Brome Land Management System

Copyright @ $@ 2008$ by

Sean Phillip Murphy 
The report of Sean Phillip Murphy is approved.
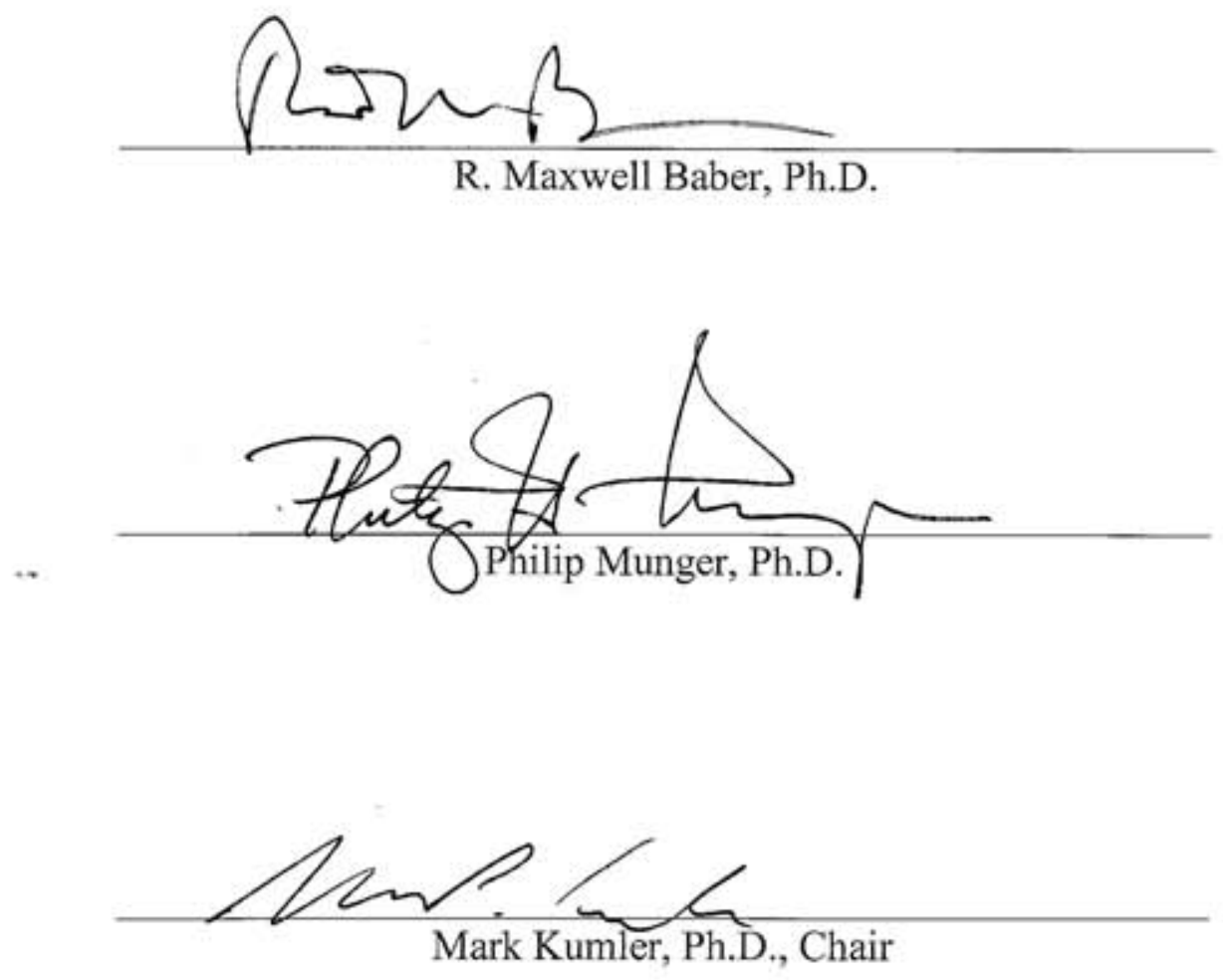

July 2008 



\section{ACKNOWLEDGEMENTS}

Mom and Dad, thank you for your support, motivation, and constant interest. You understood when I cut many phone conversations short because I had a model to build, run, or document. You taught me to set and strive for goals in what I do, become, and practice. My undergraduate advisor, Dr. Paula Sanchini, Coe College, thank you for the introducing me to the GIS realm. My graduate advisor, Dr. Mark Kumler, thank you for your support and advice. You were there from start to finish. Dr. Philip Munger, Dr. Jennifer Vollmer, and the other key-players at BASF, thank you for the opportunity to compile, execute, and document a project that will improve the world and will not sit on a bookshelf collecting dust. Lynn Flewelling, thank you for editing and streamlining my mammoth document. Brandon Davis, thank you for all the technical support. Whether it was software bugs or hardware failures, you were ready to help, troubleshoot, and reformat. Theresa Ellis, thank you for making sure the program's logistics, no matter how large or small, were planned, scheduled, and carried through. I would have been lost if it wasn't for your help. Heidi Esh and Charles Armstrong, thank you for the guidance your previous work provided. Thank you committee members: Dr. Mark Kumler, Dr. R. Maxwell Baber, and Dr. Philip Munger. Most importantly, thank you to my beautiful and lovely wife, Britta. You accompanied me to California, supported me in difficult times, and were always sympathetic to my odd hours, crankiness, and stress. You are truly my soul-mate. 



\begin{abstract}
Integrating an Agent-based Model into a Web-Enabled Annual Brome Land Management System
\end{abstract}

\author{
by \\ Sean Phillip Murphy
}

The natural fire cycle in the Great Basin area of Nevada has shortened from every 50 to 60 years to 3 to 5 years, putting many natural ecosystems and occupied lands in danger. The spreading phenomenon of the invasive annual brome will be investigated to quantify this fire risk. It is renowned for its invasive nature, flammability, and the detrimental effects it has on native annual and perennial grasses. Based on vegetation classifications and dispersal characteristics, the rules for an agent-based model will be used to simulate the future extents. Agent Analyst software in conjunction with ArcGIS will integrate simulation results into a web-enabled decision support system for land managers. 



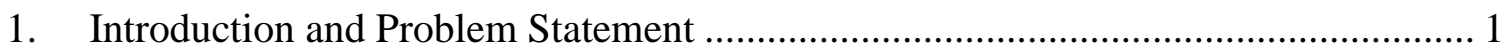

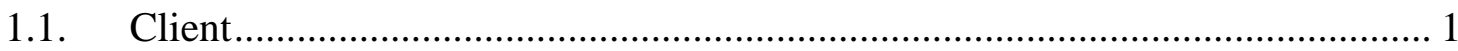

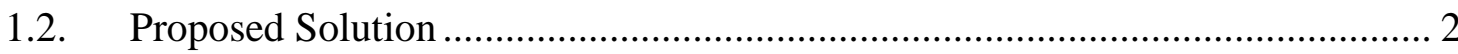

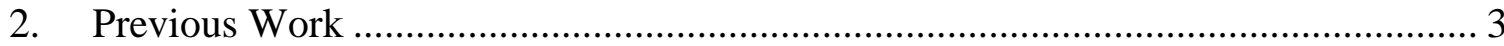

2.1. The Work of Heidi Esh .......................................................................... 3

2.2. The Work of Charles Armstrong …………................................................... 3

3. Background and Literature Review ……………............................................ 5

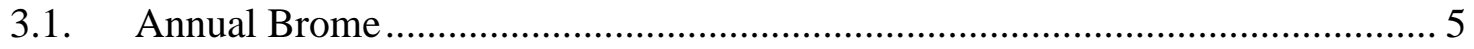

3.2. Modeling Dynamic Processes .................................................................... 5

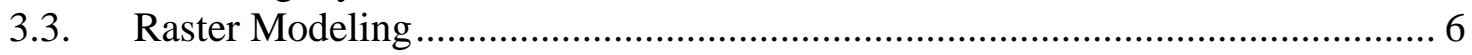

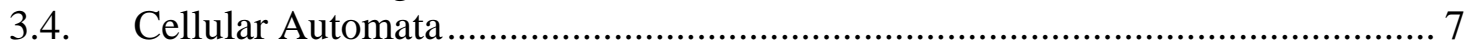

3.5. Agent-based Modeling and Simulation ........................................................ 8

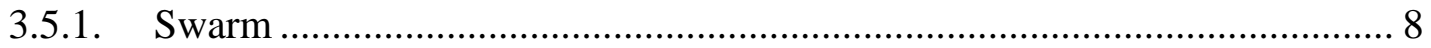

3.5.2. Starlogo TNG: The Next Generation........................................................ 9

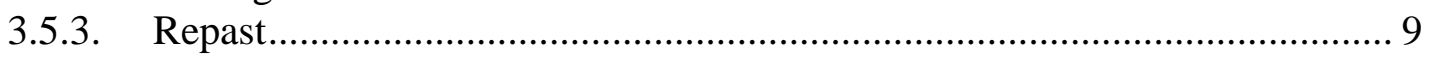

3.5.4. Agent Analyst .................................................................................... 10

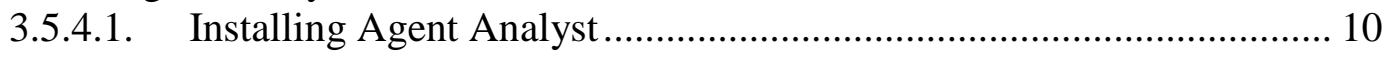

3.5.4.2. Agent Analyst Graphical User Interface .......................................... 11

3.5.4.3. Configuring Agent Analyst ................................................................ 11

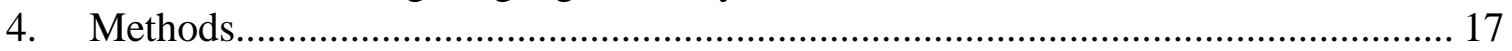

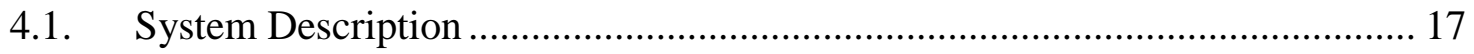

4.2. Prototype Area Description..................................................................... 17

4.3. System Architecture ............................................................................... 19

4.3.1. Software and Hardware Requirements …………................................... 20

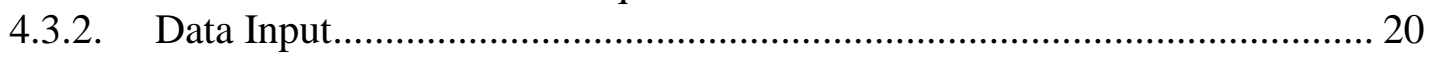

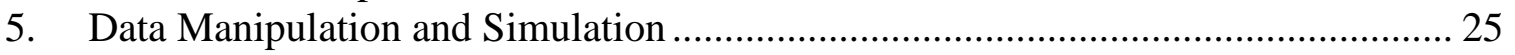

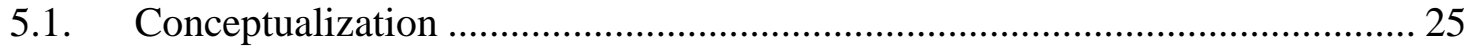

5.1.1. Rule One: The Influence of Wind.......................................................... 25

5.1.2. Rule Two: The Influence of Vegetation Density ……………………........ 25

5.1.3. Rule Three: The Influence of Automobiles ................................................ 27

5.1.4. Rule Four: Germination Probability ……………….................................. 27

5.1.5. Properties of an Agent-based Simulation ................................................. 27

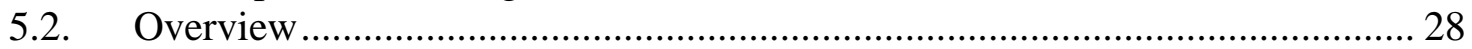

5.2.1. Customizing Data to the User Defined Extents ........................................ 29

5.2.2. Customizing Data for the Study Area ....................................................... 29

5.2.3. Attribute Calculations ........................................................................ 30

5.2.3.1. Habitat Suitability Model ............................................................... 31

5.2.3.2. Seed Density Calculation...................................................................... 36

5.2.3.3. Plant Density Calculation ................................................................ 44

5.2.4. Customizing Data for Subset Area ……………....................................... 46

5.3. Preparing Data for Agent Analyst............................................................... 46

5.3.1. Calculating Number of Agents ............................................................. 47

5.3.2. Creating Agents ..................................................................................... 49

5.3.3. Clean Up Dataset/Make Computationally More Efficient........................... 50 


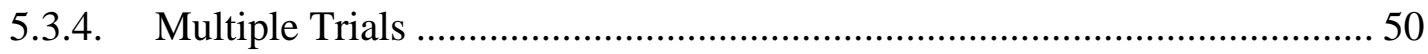

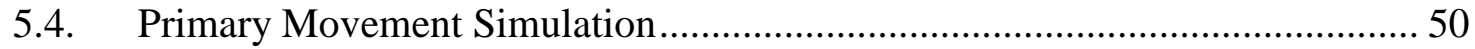

5.4.1. Configuring Agent Analyst.................................................................. 50

5.4.2. Primary Movement Simulation: Code for Agent Actions ........................... 51

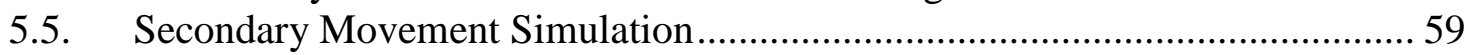

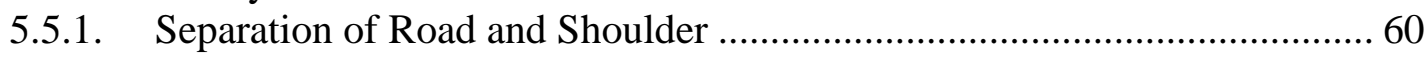

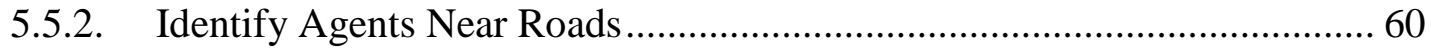

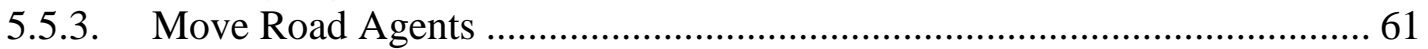

5.5.4. Combine Road Agents With Non-Road Agents ......................................... 62

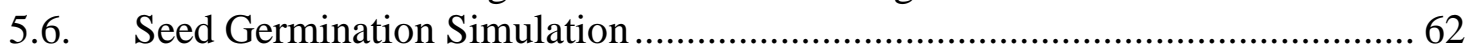

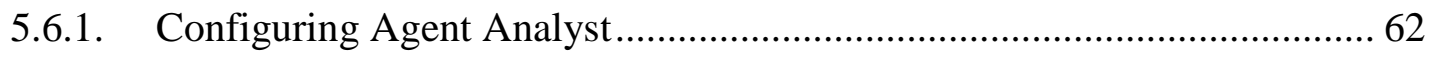

5.6.2. Seed Germination Simulation: Code for Agent Actions............................... 64

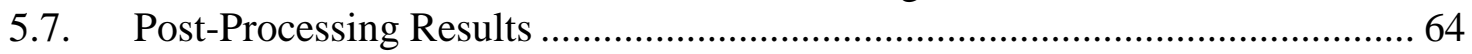

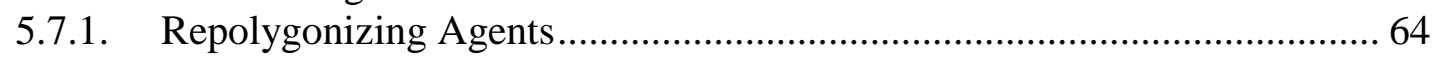

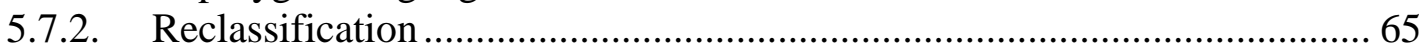

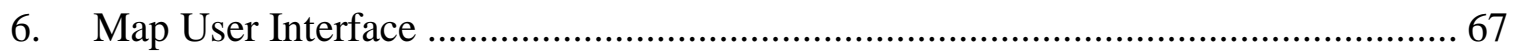

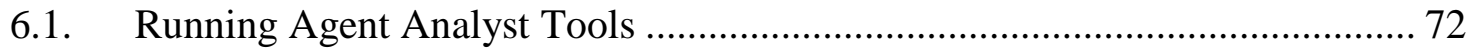

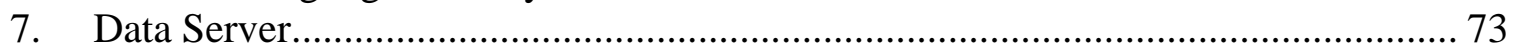

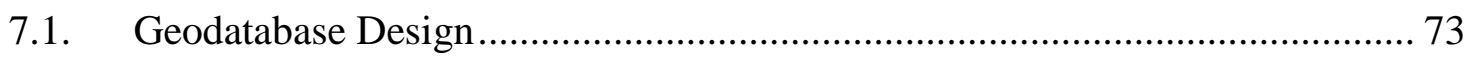

7.2. Data Layer vs. Real-time Geoprocessing......................................................... 74

7.3. Modifications to Armstrong's Base Map....................................................... 74

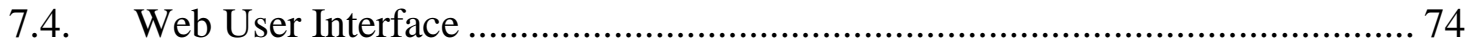

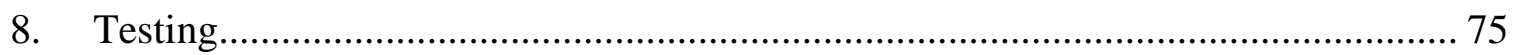

8.1. Unit Testing - Troubles with ArcGIS Desktop ............................................. 75

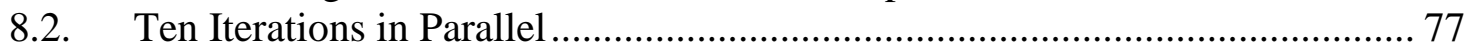

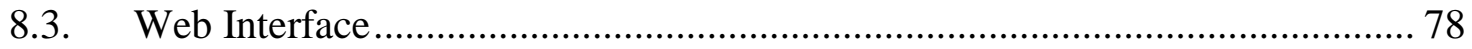

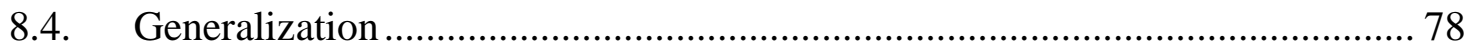

8.4.1. Ensuring No Agent Overload.............................................................. 78

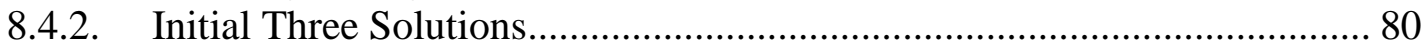

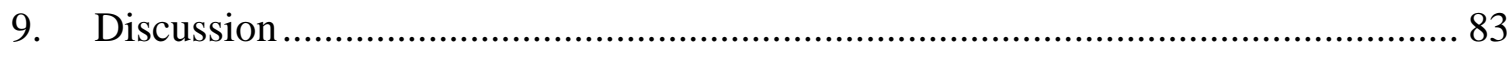

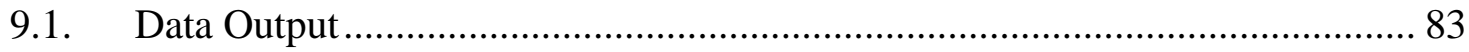

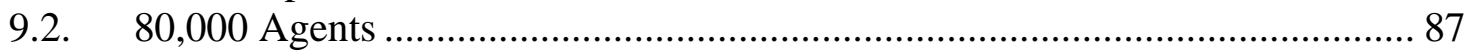

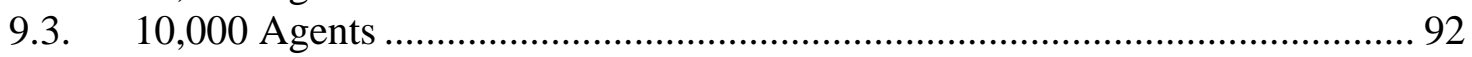

9.4. Early Development: 250,000 Agent Maximum.............................................. 95

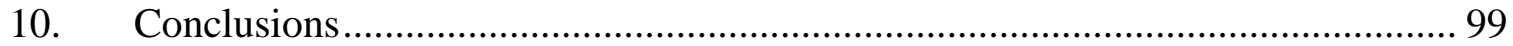

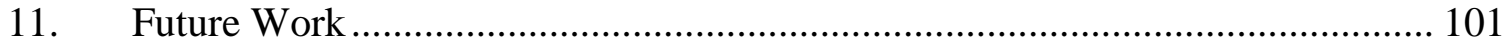

11.1. Is it Possible Using Only ArcGIS Desktop? ................................................ 101

11.2. Real-Time Running Model ..................................................................... 101

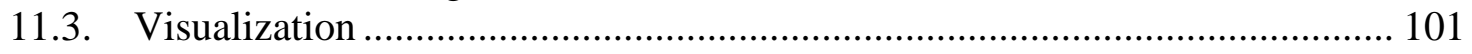

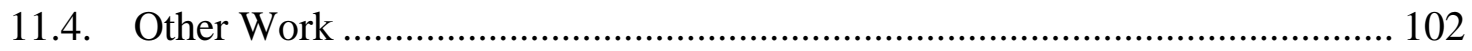

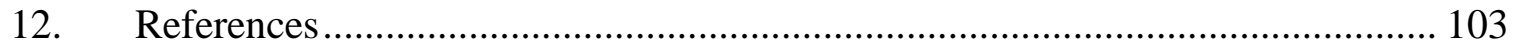

Appendix A - Agent-based Modeling Supplement Materials ........................................ 107

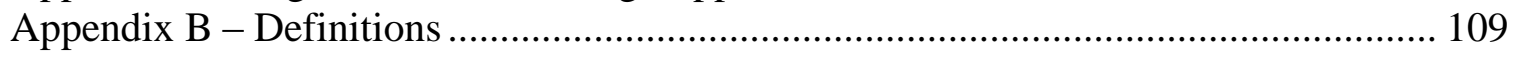

Appendix C - Seeds per Meter Calculation Model ........................................................ 111

Appendix D - Plants per Meter Calculation Model........................................................ 113

Appendix E - Calculating Number of Agents .......................................................... 115 


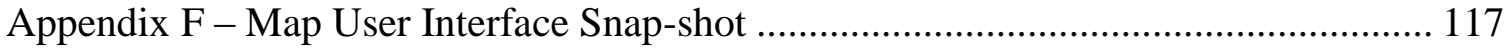

Appendix G - Calculate a Field Based on a Field's Sum Code .................................... 119

Appendix H - Code Variable Definitions................................................................. 121

Appendix I - Primary Movement Code...................................................................... 123

Appendix J - Concept for Programming Change of X and Y ................................... 125

Appendix K - Separation of Road and Shoulder Model ........................................... 127

Appendix L - Germination Simulation Code ......................................................... 129

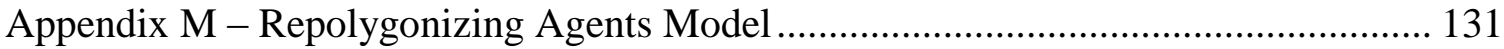

Appendix N - Reclassification Model .................................................................... 133

Appendix O - Web Application Information and Snap-shots .................................... 135 


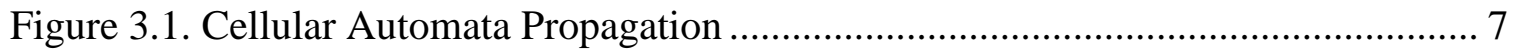

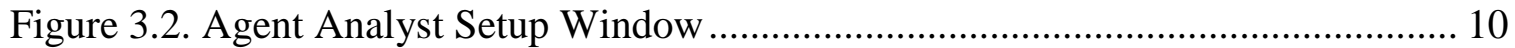

Figure 3.3. The Agent Analyst GUI - Red Arrows Left to Right: TOC, Display, and Main

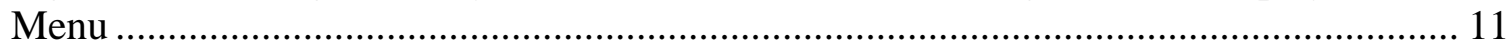

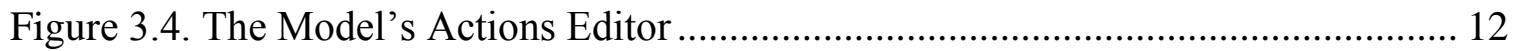

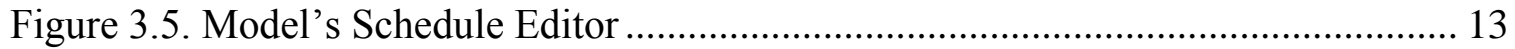

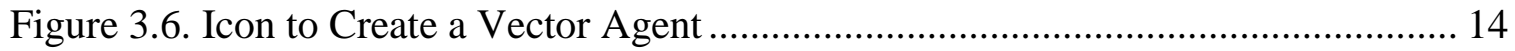

Figure 3.7. A Vector Agent's Data Source Editor .................................................. 14

Figure 3.8. A Vector Agent's Actions Editor ........................................................... 15

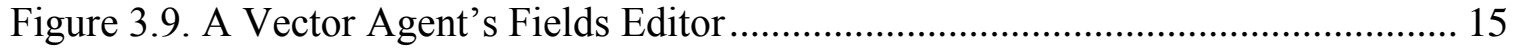

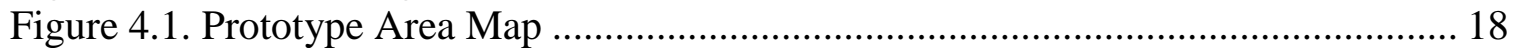

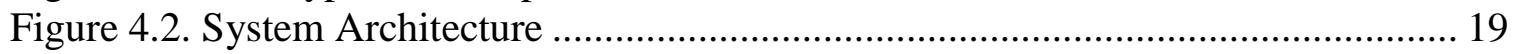

Figure 4.3. Calculating Midpoints and Attaching Line Length Attribute....................... 23

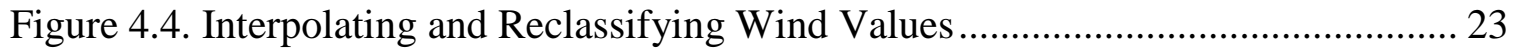

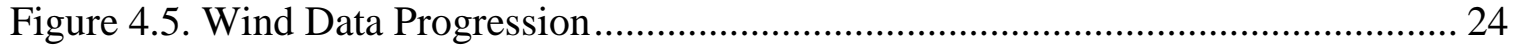

Figure 5.1. Sagebrush Community in Washoe, Nevada (Sagebrush Bird Conservation

Network, N.D.) ............................................................................................... 26

Figure 5.2. Annual Brome Grassland Community (Sagebrush Bird Conservation

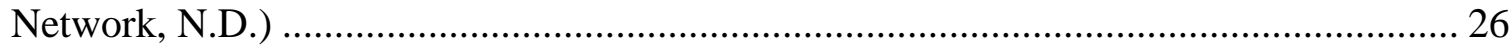

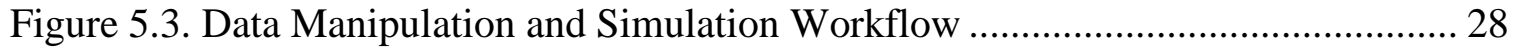

Figure 5.4. Phases of the Customizing Data to the User Defined Extents....................... 29

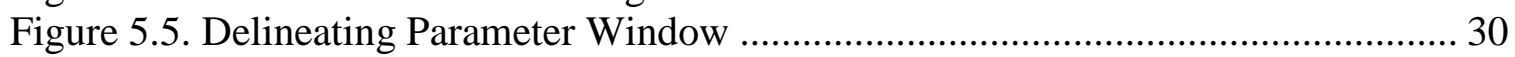

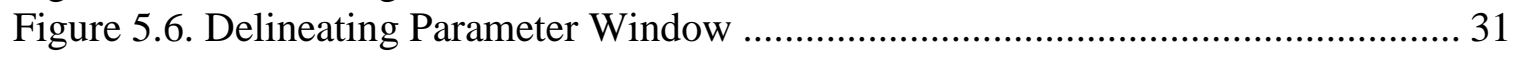

Figure 5.7. Euclidean Distance and Reclassify Tools and the Dataset Produced............. 32

Figure 5.8. Weighted Overlay Tool and its Parameters ............................................... 36

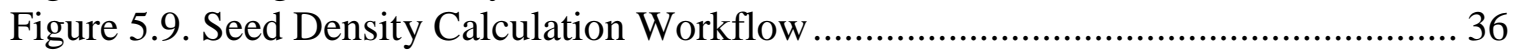

Figure 5.10. The Correlation between Plant and Seed Density .................................... 37

Figure 5.11. The Correlation between Percent Cover and Plant Density ........................ 38

Figure 5.12. The Correlation between Percent Cover and Seed Density for Values Less

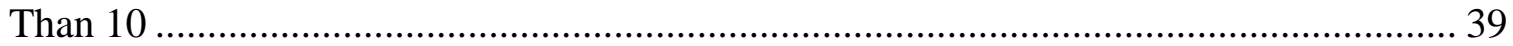

Figure 5.13. Single Output Map Algebra Tools and their Parameters............................ 40

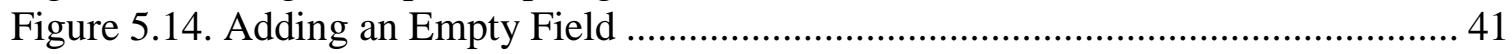

Figure 5.15. Excluding Outliers Geoprocessing String ......................................... 42

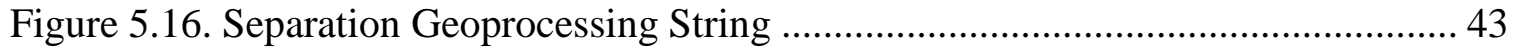

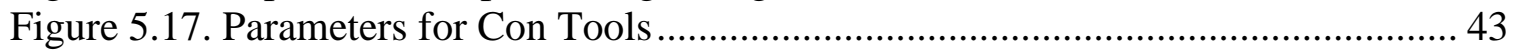

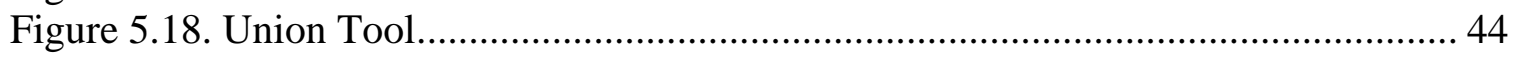

Figure 5.19. Plant Density Calculation Workflow....................................................... 44

Figure 5.20. Single Output Map Algebra Tool and Its Parameters ............................... 45

Figure 5.21. Subset Area Parameter Form for Delineation ........................................ 46

Figure 5.22. Preparing Data for Agent Analyst Workflow............................................ 47

Figure 5.23. The Role of the Calculate Areas Script ............................................... 48

Figure 5.24. Create Random Points Tool............................................................ 49

Figure 5.25. Primary Movement Simulation Workflow ............................................. 50

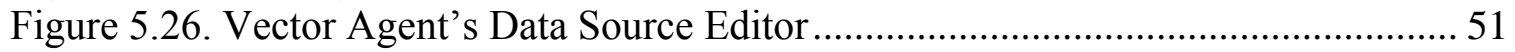




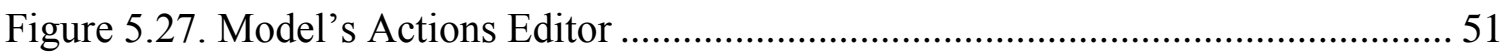

Figure 5.28. Vector Agent's Actions Editor .............................................................. 52

Figure 5.29. Correlation between Distance and Seeds Collected .................................... 54

Figure 5.30. Correlation between Distance and Standard Value ...................................... 55

Figure 5.31. Calculating Seed Distance Implemented in the Action Editor..................... 56

Figure 5.32. Calculating Seed Travel Direction Implemented in the Action Editor ........ 57

Figure 5.33. Calculating Change in X and $\mathrm{Y}$ Implemented in Actions Editor ................. 58

Figure 5.34. Calculating Final X and Y Coordinate in Actions Editor............................ 59

Figure 5.35. Secondary Movement Simulation Work Flow ……………………….......... 59

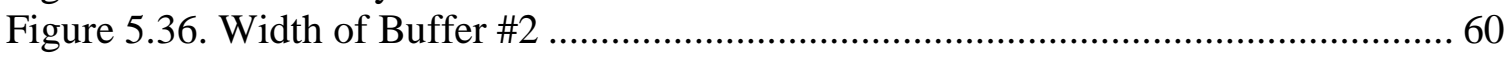

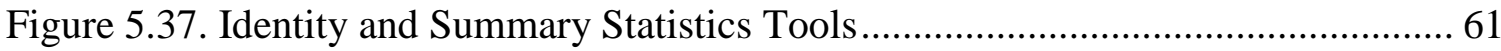

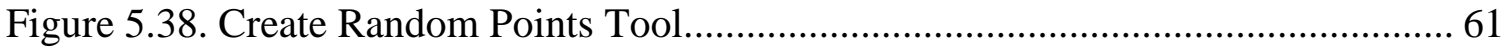

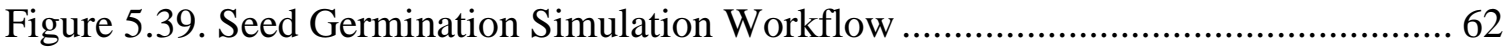

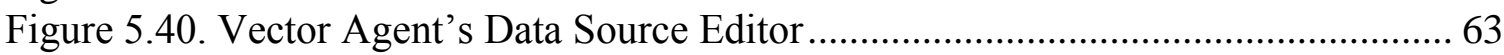

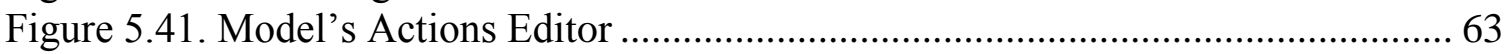

Figure 5.42. Post-Processing Results Workflow ............................................................. 64

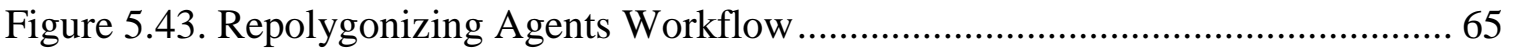

Figure 5.44. Expression the Calculates Percent Cover from Summary............................ 65

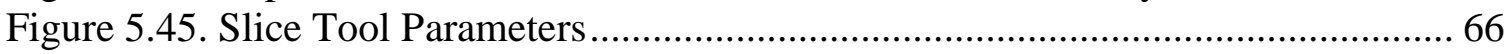

Figure 6.1. ArcMap's Table of Contents and ArcToolbox .............................................. 67

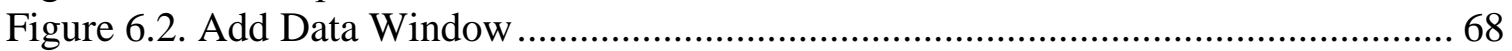

Figure 6.3. Symbolized Annual Brome Cover for 2001 ................................................. 68

Figure 6.4. Symbolized Annual Brome Cover for 2001 - Presence/Absence................... 69

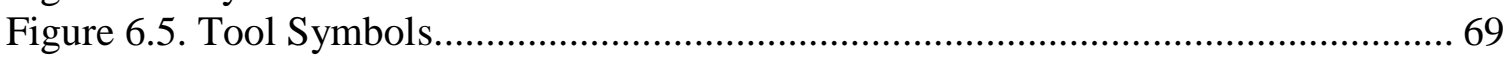

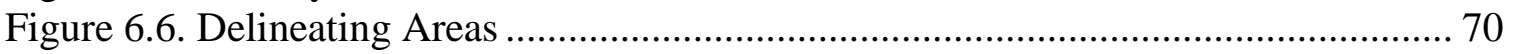

Figure 6.7. Importing Symbology for Tool Output .................................................... 70

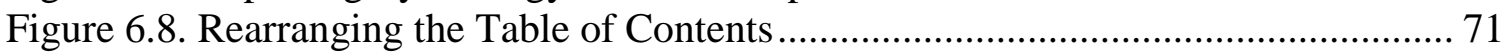

Figure 6.9. Initiate Agent Analyst Simulation Icon .................................................... 72

Figure 7.1. Data Server Geodatabase Design ............................................................... 73

Figure 8.1. Setting Geoprocess to Overwrite Output..................................................... 75

Figure 8.2. Correlation between Averaged Simulations in Parallel and Raw Maximum

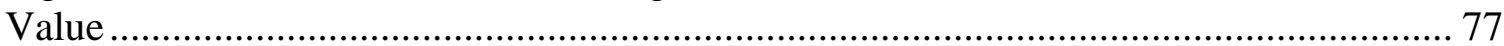

Figure 8.3. Attribute Table with Values That Result in Agents Highlighted ................... 80

Figure 9.1. Annual Brome Presence/Absence for 80 Thousand Agents........................... 88

Figure 9.2. Annual Brome Percent Cover for 80 Thousand Agents ................................ 89

Figure 9.3. Percent Cover Values Plotted Against the Number of Cells Representing

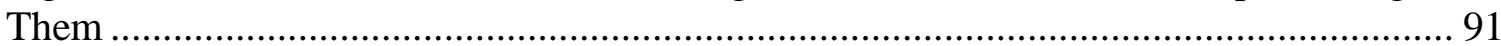

Figure 9.4. Percent Cover Values Plotted Against the Number of Cells Representing

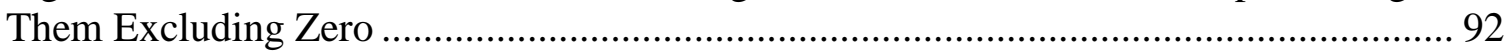

Figure 9.5. Annual Brome Presence/Absence for 10 Thousand Agents........................... 93

Figure 9.6. Annual Brome Percent Cover for 10 Thousand Agents ................................ 94

Figure 9.7. Annual Brome Presence/Absence for 250 Thousand Agents......................... 96

Figure 9.8. Annual Brome Percent Cover for 250 Thousand Agents .............................. 97 
Table 2.1. Annual Brome Habitat Suitability Parameters (Esh, 2006) ............................ 3 Table 2.2. Annual Brome Habitat Suitability Parameters - A Comparison between Esh (2006) and Bradley and Mustard (2006).................................................................... 4

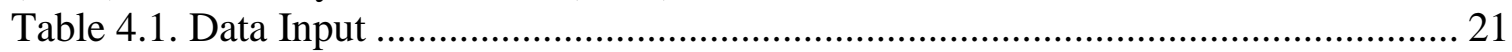

Table 5.1. Power Line Distance Reclassification Schema........................................... 32

Table 5.2. 1973 Annual Brome Cover Distance Reclassification Schema...................... 33

Table 5.3. Cultivation Distance Reclassification Schema ............................................ 33

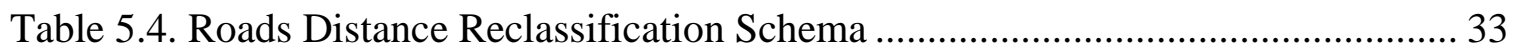

Table 5.5. Hydrology Distance Reclassification Schema ........................................... 34

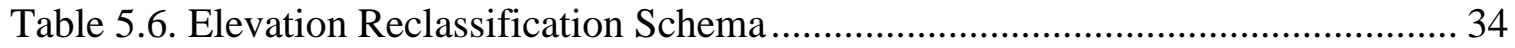

Table 5.7. Surface Aspect Reclassification Schema ..................................................... 35

Table 5.8. Habitat Suitability Weighted Overlay Parameters ......................................... 35

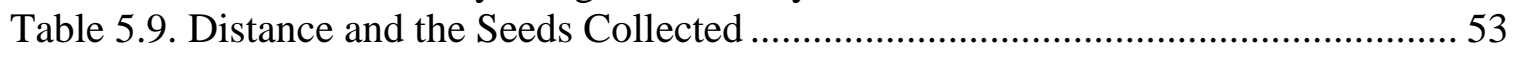

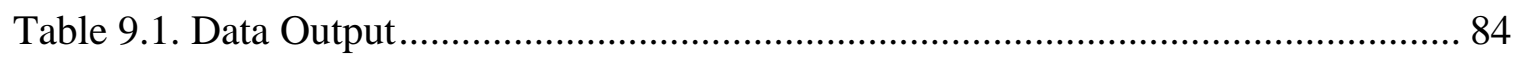

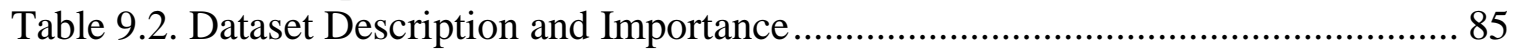

Table 9.3. Presence Statistics for 80 Thousand Agents ............................................ 90

Table 9.4. Presence Statistics for 10 Thousand Agents ................................................ 95

Table 9.5. Presence Statistics for 250 Thousand Agents ............................................ 98 


$\begin{array}{ll}\text { AA } & \text { Agent Analyst } \\ \text { ABM } & \text { Agent-based Modeling } \\ \text { ABMS } & \text { Agent-based Modeling and Simulation } \\ \text { AOI } & \text { Area of Interest } \\ \text { CA } & \text { Cellular Automata } \\ \text { CAS } & \text { Complex Adaptive System } \\ \text { DEM } & \text { Digital Elevation Model } \\ \text { GHz } & \text { Gigahertz } \\ \text { GIS } & \text { Geographic Information Systems } \\ \text { GUI } & \text { Graphical User Interface } \\ \text { ICBEMP } & \text { Interior Columbia Basin Ecosystem Management Project } \\ \text { IIS } & \text { Internet Information Server } \\ \text { IPM } & \text { Integrated Pest Management } \\ \text { MIP } & \text { Major Individual Projects } \\ \text { NDVI } & \text { Normalized Difference Vegetation Index } \\ \text { NvNHP } & \text { Nevada Natural Heritage Program } \\ \text { RAM } & \text { Random Access Memory } \\ \text { Repast } & \text { Recursive Porous Agent Simulation Toolkit } \\ \text { RI } & \text { Redlands Institute } \\ \text { ROAD } & \text { Repast Organization for Architecture and Development } \\ \text { TNG } & \text { The Next Generation } \\ \text { USDA } & \text { United States Department of Agriculture } \\ \text { WISP } & \text { Weed Invasion Susceptibility Prediction } \\ \text { WUI } & \text { Wildland Urban Interface }\end{array}$





\section{Introduction and Problem Statement}

A drought in the winter and spring of 2007 created dry vegetation during the summer months throughout the Western US. This led to 888 fires that burned more than 890,000 acres of forest and rangeland (Klug, 2007). The Nevada Division of Forestry states that, before 1999, a significant fire season would occur on average every twenty years, burning 300,000 acres. Since 1999, there has been an increase in magnitude and frequency of bad fire seasons; the last six fire seasons have averaged over one million acres of burned land (Klug, 2007).

Land managers commonly focus on protecting and managing forests, ignoring other flammable vegetation types. However, only nine percent of large fires in the 2006 fiscal year burned timber, whereas 67 percent burned non-timber vegetation, such as grass and brush (The Wilderness Society, 2006). During a single event in 2007, six fires burned 653,000 acres of grass and sagebrush on the Nevada-Idaho border (Frederick, 2007).

Annual brome contributes a large portion of the fine fuel accumulation in grassland and sagebrush communities it has invaded. Its monocultures mature and become dry in the summer, accumulating $2.9 \mathrm{~g}$ of dry matter per sq $\mathrm{mm}$ a day (Neese, 2000). This high quotient of fuel accumulation makes it ideal for wildfires, often increasing both the intensity and rate-of-spread (Zouhar, 2003). The annual brome complex, common name cheatgrass, includes Bromus diandrus (ripgut brome), B. rubens (red brome), B. secalinus (rye brome), and B. tectorum (downy brome). In this document, "annual brome" will refer to this group of annual grasses.

Geographic Information Systems (GIS) can conduct spatial analyses in plant ecology. With the advances in modern computer hardware and software, the increase in geographic data accuracy, and the progression of scientific literature, more sophisticated habitat prediction methods are becoming available. This means land managers can now have highly accurate and comprehendible models on which to base their fire prevention strategies.

\subsection{Client}

The client for this project is the BASF Corporation via Dr. Phil Munger, a Senior Biology Area Manager. BASF is the world's largest chemical company. It is based in Europe with sites in the USA, Asia, and South America. The corporation creates a wide array of organic chemicals, which includes the herbicide Plateau (BASF, 2008).

The client helped develop a realistic scope achievable for this project, and in doing so, outlined their long term expectations. BASF's goal is to create a marketing tool for Plateau by providing potential customers with a land management tool that is geared towards protecting their land.

The client expects this web-enabled decision support system to incorporate components of agent-based modeling (ABM). By adding ABM to this management tool, land managers, owners, and insurers can more accurately decide proper precautions. BASF's marketing department will be available to all parties, answering all questions about how Plateau can help reduce the severity of annual brome presence and growth. To fulfill 
these expectations, a prototype model was created, which extends data coverage through northwest Nevada.

\subsection{Proposed Solution}

Because an array of environmental and anthropogenic factors have led to the erratic and worsening issue of annual brome spread, a dynamic spatial model was created to predict the future range. The information that these simulations provide can then be acquired by an end-user through a published thin-client web service. Since the groundwork for the web-enabled land management system was set by Charles Armstrong (2007), this project will focus on the integration of Agent-based Modeling and Simulation (ABMS).

Simulating the future range of an annual plant requires knowledge of its reproduction dynamics, accounting for seed and environment interactions. How does a seed move from the parent plant to a new location? Once a seed has reached a new location, what is the probability of that seed germinating in that microenvironment? To answer both of these questions, scientific literature about seed migrations and habitat suitability parameters formed the basis for the simulation.

Users who wish to utilize ABMS must be knowledgeable in advanced object-oriented programming. Well thought-out tutorials for the novice user are hard to find; thus the field of ABMS has remained largely unexplored.

To reduce the learning curve, a goal of this project is to implement an interface, called Agent Analyst, between an ABMS platform called Repast and ArcGIS Desktop. While ArcGIS Desktop has gained a reputation for lacking simulation functionalities, it has proven to be very useful as a data manager, data transformer, data analyzer, and creator of symbology. Using Agent Analyst, simple agent rules can be applied to a geographic dataset and create simulated changes within the data.

This project sets out to not only to improve on a decision tool for annual brome management, but also as an exploratory study to exhibit the power of an ArcGIS Desktop and Agent Analyst partnership. The proposed solution incorporates a series of geoprocessing models composed in ModelBuilder, simple code written in the Agent Analyst, and files and folders organized in ArcCatalog.

In order to dovetail with the previous work of Esh (2006), Armstrong (2007), Peterson (2003), and Bradley and Mustard (2006), this project's prototype area was set to central and northern Nevada. The habitat suitability geoprocessing model developed by Armstrong was incorporated into this project's agent-based model. End-users can view habitat suitability alongside the agent-based results allowing them to compare and contrast current and future annual brome presence. 


\section{Previous Work}

As stated in the previous sections, this study will improve on two Major Individual Projects. To understand this project's context, both previous projects will be summarized and related to this project.

\subsection{The Work of Heidi Esh}

Heidi Esh was a pioneer in creating a habitat suitability model and high-fire danger model for BASF (Esh, 2006). She chose to focus her prototype on the Wildland Urban Interface (WUI) surrounding the community of Big Bear, California. The prototype's primary objectives were to identify locations of high-fire hazard, predict fire behavior, and locate potential areas for fuel break installation. She separated her model into two different parts: the first used environmental parameters to define areas of estimated cheatgrass domination; the second referenced the United States Department of Agriculture (USDA) Forest Service and Mike Price (2005) at ESRI to quantify vegetation combustibility.

The habitat suitability model was of particular interest. The parameters Esh incorporated into her model supported the work of experts at ESRI, the USDA Forest Service, and a thesis by Gillham (2001). Charles Armstrong (2007) criticized the parameters used by Esh (see Table 2.1), stating that the model focuses largely on invasive plants and does not apply directly to annual brome, referring to Gillham (2001) WISP (Weed Invasion Susceptibility Prediction) as an example.

Table 2.1. Annual Brome Habitat Suitability Parameters (Esh, 2006)

\begin{tabular}{|l|l|}
\hline Aspect & S \& W \\
\hline Elevation & $\begin{array}{l}\text { More than } 762 \\
\text { meters }\end{array}$ \\
\hline Fire History \\
\hline Fuel Reduction Zones \\
\hline Roads & Within 180 meters \\
\hline Urban Areas & Within 180 meters \\
\hline
\end{tabular}

\subsection{The Work of Charles Armstrong}

Charles Armstrong incorporated a habitat suitability model with a web accessible geoprocessing task (Armstrong, 2007) by developing a thin-client web-enabled GIS decision support system, using the ArcGIS Server template. Each end-user can open the web application, the home of the map service, where an Area of Interest (AOI) can then be defined in the graphical user interface (GUI). This AOI is then sent to the internet server that hosts the web application, where the habitat suitability geoprocessing model runs for the user-defined extent. The server returns the results to the client's internet browser. Armstrong includes most of central Nevada as his prototype area (the maximum area AOI can be defined), reasoning that expanding the model to intermountain areas could easily be done if the correct datasets were available.

Armstrong also took the first steps in incorporating the data from other web applications called web portals. First, he connected with wildland urban interface (WUI) data for 
1990-2000, served by the SILVIS Lab Forest Ecology and Management University of Wisconsin, Madison web portal. He then connected to data on fire behavior with various fuels and fire regimes, served by the USDA Forest Service Landfire project. Both web portals prove to be useful in land management decisions, but Armstrong notes that for the user to utilize the full potential of web GIS, the data portals need to not only be viewed in a web application but used as inputs in real-time geoprocessing. He goes on to speculate that this is a fault of ArcGIS Server 9.2 and may be corrected with the release of 9.3.

As stated in Chapter 2, Armstrong diverged from Esh in deciding annual brome habitat parameters used in his geoprocessing model. Armstrong chose to follow the work of Bradley and Mustard (2006), who determined relationships between annual brome and certain landscape features by using the Normalized Difference Vegetation Index (NDVI) to classify Landsat data (see Table 2.2). Bradley and Mustard calibrated their classifications with in-field verification.

Table 2.2. Annual Brome Habitat Suitability Parameters - A Comparison between Esh (2006) and Bradley and Mustard (2006)

\begin{tabular}{|l|l|l|l|}
\hline \multicolumn{2}{|c|}{ Esh (2006) } & \multicolumn{2}{c|}{ Bradley and Mustard (2006) } \\
\hline Aspect & S \& W & Aspect & W \& NW \\
\hline Elevation & $\begin{array}{l}\text { More than 762 } \\
\text { meters }\end{array}$ & Elevation & $\begin{array}{l}\text { Between 1400 \& } \\
1700 \text { meters }\end{array}$ \\
\hline Fire History & N/A & \\
\hline Fuel Reduction Zones & W/A & Within 700 meters \\
\hline Roads & Within 180 meters & Roads & (Part of Cultivation) \\
\hline Urban Areas & Urban Areas & Hydrologic Channels \\
\hline N/A & N/A & Cultivation & Within 3000 meters \\
\hline Cultivation & N/A & Power Lines & Within 1000 meters \\
\hline $\begin{array}{l}\text { Power Lines } \\
\text { Cheatgrass } \\
\text { History }\end{array}$ & N/A & $\begin{array}{l}\text { 1973 Annual } \\
\text { Brome Cover }\end{array}$ & Within 150 meters \\
\hline
\end{tabular}




\section{Background and Literature Review}

\subsection{Annual Brome}

The annual brome complex consists of invasive monocot grasses that infiltrate and dominate nonnative landscapes. Its origin spans Europe, Africa, and Asia and it has been introduced to North and South America (Novak \& Mack, 2001; Updadhyaya, Turkington, \& McIlvride, 1986; Young, 2000; Zouhar, 2003). The early invasions originated from crate packing material, and first became established near rail lines (BASF, 2006).

The successful nature of an annual brome community can be attributed to its ability to produce 20 to 30 thousand seeds per square meter. The barbed seeds gain access to miles of virgin territory by wind, or by adhering to car tire treads, clothing, and domestic and wild animals (Hulbert, 1955; Neese, 2000). With these means of spread, it has expanded to cover nearly 25 million acres in the Great Basin, 100 million acres nationwide, and is further spreading at a rate of 2,000 acres a day (BASF, 2006).

Annual brome typically grows in monocultures - pure stands - often suffocating native grasses and other vegetation types (Young, 1978; Zouhar, 2003). Its roots have the advantage of growing throughout the winter season, out-competing native perennial species in early spring (BASF, 2006). In competition experiments, annual brome has shown high rates of nutrient uptake and growth (Booth, Caldwell, \& Stark, 2003) and exhausts soil moisture rapidly (Harris, 1967; Zouhar, 2003), explaining why many slower-growing shrub, brush, and bunchgrass communities have been replaced. Overgrazed, overcultivated, or burned grass, sage brush, bitterbrush, ponderosa pine and Douglas-fir ecosystems are also susceptible to invasion (Zouhar, 2003).

\subsection{Modeling Dynamic Processes}

Longley and others (2005) assert that all entities of the world are complex.

Representations create a simplified manner in which our world and geographic

knowledge can be assembled. Models of these representations usually aid research, are a tool for clarification, simulation, and prediction, and are an integrator between disciplines (Mulligan \& Wainwright, 2004).

Ecological models contain two properties that must be established: the study area size to work within and the complexity (Perry \& Bond, 2004). Ecological complexity must be analyzed and constantly updated as science progresses, especially the dynamic cycles within nature (Mulligan \& Wainwright, 2004). Representations of the infinitely complex natural life cycles often appear simpler than reality. For example, in ecology, not only is there biological predation and competition, but the physical environment influences biotic and abiotic interactions (Perry \& Bond, 2004).

Current technology is limited to algorithms and statistical formulas, influencing the deviations between reality and its representation (Heuvelink, 2000). Therefore a model that has the greatest explanation and predictive power yet has the least parameters and complexity, known as the parsimonious model (Mulligan \& Wainwright, 2004), is ideal.

GIS allows analysts to create both simple and complex models without a complete knowledge about the applied algorithms. For example, Beckler, French, \& Chandler 
(2005) created an integrated pest management model for corn rootworm beetles and soil types by interpolating field data, building a contingency model, and analyzing spatial autocorrelation with Moran's I coefficient. In their analysis, they input the data required and exclude extraneous field data. By doing this, they created a parsimonious model that directly pointed out relationships between beetle emergence and soil types.

GIS works with data containing information about a specific place at a specific time, creating a static representation for data analysis. However, time can be broken into steps called iterations. Each iteration can have its own set of operations that are carried out on the dataset (Longley et al., 2005). This manner in which the computer and geography interact, often called dynamic geocomputational models (Longley et al., 2005), has evolved over the last few decades.

\subsection{Raster Modeling}

The advantages of breaking down geographic features into a lattice have been researched since Tobler (1979) introduced the idea of cellular geography. He described how certain cells can have a relationship with spatial and temporal neighbors or have no relationship. For example, a land use attribute in the current year may have been influenced by the previous year's use or a spatially close or inherent variable.

The paper ,"Raster Modeling in GIS", by Bitterlich, Alsawydami, and Douglas (1993) shows how "raster models can be used to simulate dynamic phenomena where an activity starts at some points (locations), at a given time, and continues to spread via interaction with its neighbors". They go on to classify raster model applications in GIS into two categories: diffusion models and cellular automata.

Diffusion models are used in dynamic processes that involve flow configurations, such as fire spread and water movement. The raster is viewed as "a sparse network with bidirectional arcs linking neighborhood pixels" (Bitterlich et al., 1993). Mathematical rules decide the change of a pixel's attribute based on the value of a mutually exclusive attribute. The first attribute changes when the value of the mutually exclusive attribute is below a certain numerical threshold, and this creates a fastest path or path of least resistance.

Dijkstra's algorithm is an example of a diffusion model. Imagine multiple vertices on a graph, having one vertex as the source. The paths between the source vertex and every other vertex are calculated, distinguishing which vertex has the least distance between itself and the source. As stated in the previous paragraph, often costs (resistances) are assigned to paths, changing the distinguished vertex. For example, if the distance between the source vertex and the secondary vertex is short but the cost is greater than a tertiary vertex with a longer distance from the source, then the path of least cost (resistance) is from source vertex to that tertiary vertex despite the larger distance. This process of calculating the path of least cost is done iteration by iteration to find the final path to a predefined vertex. This may involve multiple intermediate vertices. For an excellent web-based learning tool of Dijkstra's algorithm, refer to Laffra (N.D.). 


\subsection{Cellular Automata}

A cellular automaton is another form of raster-based dynamic modeling. John von Neumann, a biologist, originally introduced cellular automata for describing biological self-reproduction $(1963,1966)$. Since then, John Conway introduced the Game of Life (Gardner, 1970) and Wolfram (1983) explored the modeling capacities in various disciplines. Toffoli \& Margolus (1988) described cellular automaton as a "stylized universe", where its cells contain data, it is dynamic, and there are incremental laws through which new cell states are determined by the closest neighbors.

$\mathrm{CA}$ is distinguished from diffusion models by five underlying characteristics (Bitterlich et al., 1993). (1) A grid (raster) composes the structure where (2) each cell takes a finite set of possible values. (3) Each cell evolves in discrete time steps and (4) to the same rules as every other cell. (5) Which evolutionary rules a cell follows are established by its neighborhood.

In CA, a changing cell state is dependent on neighbors (Bitterlich et al., 1993). Conway's Game of Life exemplified the "von Neumann neighborhood", defined as adjacent cells. Another popular configuration is the "Moore neighborhood", defined as the eight surrounding cells (a checkerboard configuration). With a potentially infinite number of definitions for a neighborhood, a priori knowledge of the process being modeled should weigh heavily in deciding the configuration to produce the most accurate prediction.

A weakness of CA is the propagation direction at coarse simple grid resolutions. Movement distortion results from certain cell sizes and how much area those cells represent. For example, in a three-by-three grid, movements from the center cell are limited to eight directions at 45 degree increments (see Figure 3.1).

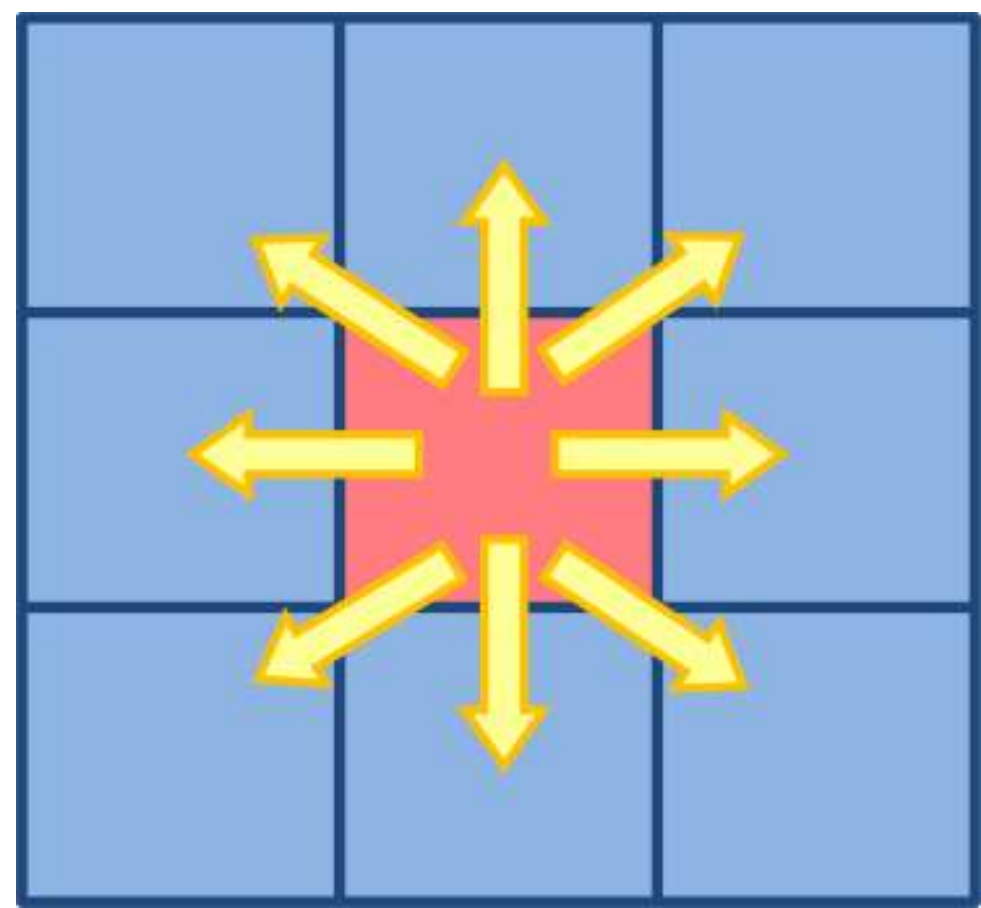

Figure 3.1. Cellular Automata Propagation 


\subsection{Agent-based Modeling and Simulation}

A system is comprised of a collection of interacting components that form what North and Macal (2007) call a complex adaptive system (CAS). To understand a system as a whole, each component's contributions, influences, and interactions must be understood. Agent-based modeling and simulation (ABMS) capture these emergent behaviors and problems presented by CAS.

The decision-making and behavioral components of CAS are called agents (North \& Macal, 2007). Each agent is characterized by attributes - indicators of what it is - and behaviors - instructions to what it does. Simple agents have four characteristics: they are adaptive; they can learn and adapt their behaviors; they are autonomous; and they produce heterogeneous results. Agents also "have sets of rules or behavior patterns that allow them to take in information, process the inputs, and then effect changes in the outside environment" (North \& Macal, 2007).

The agents are what make ABMS advantageous over cellular automata and other rasterbased complex adaptive systems. The lattice structure of these raster models represents a changing state within each cell and neglects to account for the underlying individual component (agent) that decides that state. ABMS accounts for the attributes and behaviors of each agent, and changes inter- and extra-attributes of systems that contain them.

Agent-based systems are constructed through object-oriented programming. However, because public and private researchers benefit from ABMS, open-source toolkits are also available. The difficulty in developing a simulation depends on a user's experience level. Three toolkits and one unique interface between ABMS and GIS will be discussed in the following subsections.

\subsubsection{Swarm}

Swarm was released in 1994 by Chris Langton of the Santa Fe Institute in Santa Fe, New Mexico. Swarm is an ABMS library that users and developers can incorporate into their own Java or Objective-C programs (North \& Macal, 2007). According to Gimblett (2002), the vital issue the Swarm libraries address when compared to other ABMS platforms is "the enforcement of an accepted concurrency model for the agents' actions," which allows implementing of independent behaviors from agents who are acting simultaneously from a single computer processor. The following paragraph is from the documentation released with the full beta version code in 1996:

Swarm is a multiagent software platform for the simulation of complex adaptive systems. In the Swarm system the basic unit of simulation is the swarm, a collection of agents executing a schedule of actions. Swarm supports hierarchical modeling approaches whereby agents can be composed of swarms of other agents in nested structures. Swarm provides object oriented libraries of reusable components for building models and analyzing, displaying, and controlling experiments on those models (Minar, Burkhart, Langton, \& Askenazi, 1996).

For more information and to view the free libraries, see Appendix A. 


\subsubsection{Starlogo TNG: The Next Generation}

The first full beta-edition of Starlogo TNG was released in October 2007 by the MIT Teacher Education Program in Cambridge, Massachusetts. The toolkit is based on Java and is compatible with Windows, Mac OS X, and Linux computer systems. Unlike the Swarm libraries, Starlogo TNG provides tutorials for the novice user, which include stepby-step documents and videos, and a revolutionary ABMS interface that requires minimal programming experience. According to the Starlogo TNG website (MIT Teacher Education Program, 2008), its goal is to (1) "lower the barrier of entry for programming by making programming easier," (2)“entice more young people into programming through tools that facilitate making games," and (3) "create compelling 3D worlds that encompass rich games and simulations."

Starlogo TNG is by far the most user-friendly platform for ABMS beginners. However, the simulation platform does sacrifice the flexibility seen with raw object-oriented programming and Swarm libraries. For more information and tutorials, see Appendix A.

\subsubsection{Repast}

Repast, the REcursive Porous Agent Simulation Toolkit, was originally developed at the University of Chicago and is now managed by the nonprofit volunteer Repast Organization for Architecture and Development (ROAD). The following paragraph explains the goals of ROAD and what Repast seeks to do:

Our goal with Repast is to move beyond the representation of agents as discrete, self-contained entities in favor of a view of social actors as permeable, interleaved, and mutually defining; with cascading and recombinant motives. We intend to support the modeling of belief systems, agents, organizations, and institutions as recursive social constructions (Repast Home Page, 2008).

Repast's flexibility makes it desirable among many novice and advanced users of ABMS. There are four members in the Repast collection: Repast Py (Python-based Repast), Repast J (Java-based Repast), Repast .NET (Microsoft .NET-based Repast), and Repast Simphony. Repast Py creates easy prototype agent models and can be exported to the Java language (North \& Macal, 2007). Repast J was designed to support developing large-scale agent models and includes various features unavailable in Repast Py. The Repast .NET C \# modeling environment includes all the features of Repast J but can be supported within the framework of C\#, C++, and Visual Basic. Repast Simphony encapsulates all the features of Repast $\mathrm{J}$ but extends the package by offering a point-andclick method of constructing complex agent simulations.

The use of Repast Simphony as an agent simulation constructor will no doubt increase in the future, but because it is currently in the alpha release cycle, the Repast 3 Suite (Repast Py, J, and .NET) is the most stable and was therefore preferred. The Repast 3 Suite also offers more documentation and tutorials. For more information and tutorials, see Appendix A. 


\subsubsection{Agent Analyst}

Because of the extensive documentation and flexibility of the Repast 3 Suite, extensions have been developed that create an interface between the agent-based simulation realm and GIS. Of particular interest is Agent Analyst.

The Agent Analyst toolkit was developed by Argonne National Laboratory's Center for Complex Adaptive Agent Systems Simulation Decision and Information Science Division in collaboration with ESRI. The Redlands Institute (RI) at the University of Redlands serves a web portal that conglomerates the downloadable extension, instructions on installation, tutorials, and basic information on Agent Analyst, Repast, and ABMS. On February 27, 2008, the RI uploaded Agent Analyst 1.0 beta, which is the latest version and is compatible with ArcGIS 9.2 (with Service Pack 4).

Agent Analyst integrates the functionalities of Repast with ArcGIS and ArcObjects. The interface between Repast and ArcGIS allows GIS analysts and experts to model behaviors and processes in the leading GIS software package. With Agent Analyst's graphical user interface (GUI) and ability to run in parallel with ArcGIS, users can create agents, schedule simulations, edit datasets and, most importantly, specify the behavior and interactions of agents. For more information and tutorials, see Appendix A.

\subsubsection{Installing Agent Analyst}

The latest version of Agent Analyst (AA) is a $55 \mathrm{MB}$ download. For the program to install correctly, the latest version of Java is necessary, but otherwise, the install is straightforward and simple. It requires approximately 123.4 MB of hard disk and a defined path in which to place the program files. For the annual brome agent-based model, it is recommended that the default be used (see Figure 3.2).

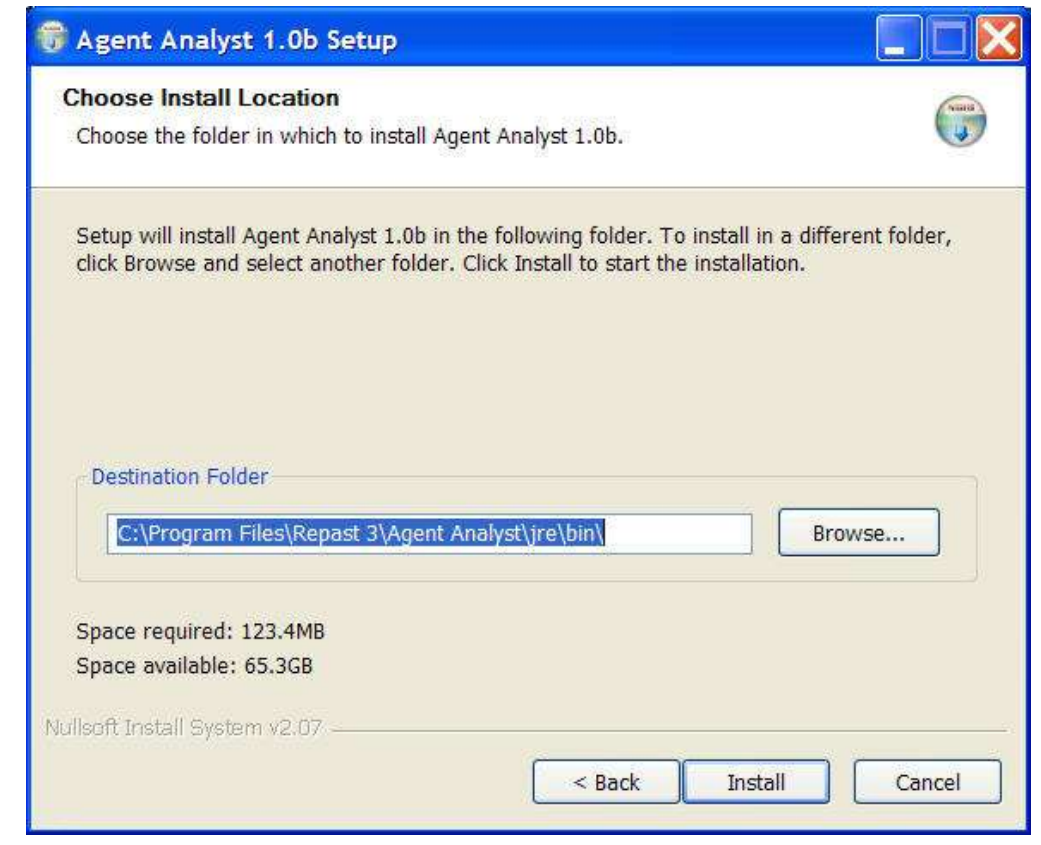

Figure 3.2. Agent Analyst Setup Window 


\subsubsection{Agent Analyst Graphical User Interface}

To start a new project, the user simply right-clicks within a custom ArcGIS toolbox, scrolls down to New, and selects Agent Analyst Tool; this starts a new edit session. The main window opens, displaying the agent-based model hierarchy - the environment, model, and agent levels - within in a simple GUI (see Figure 3.3).

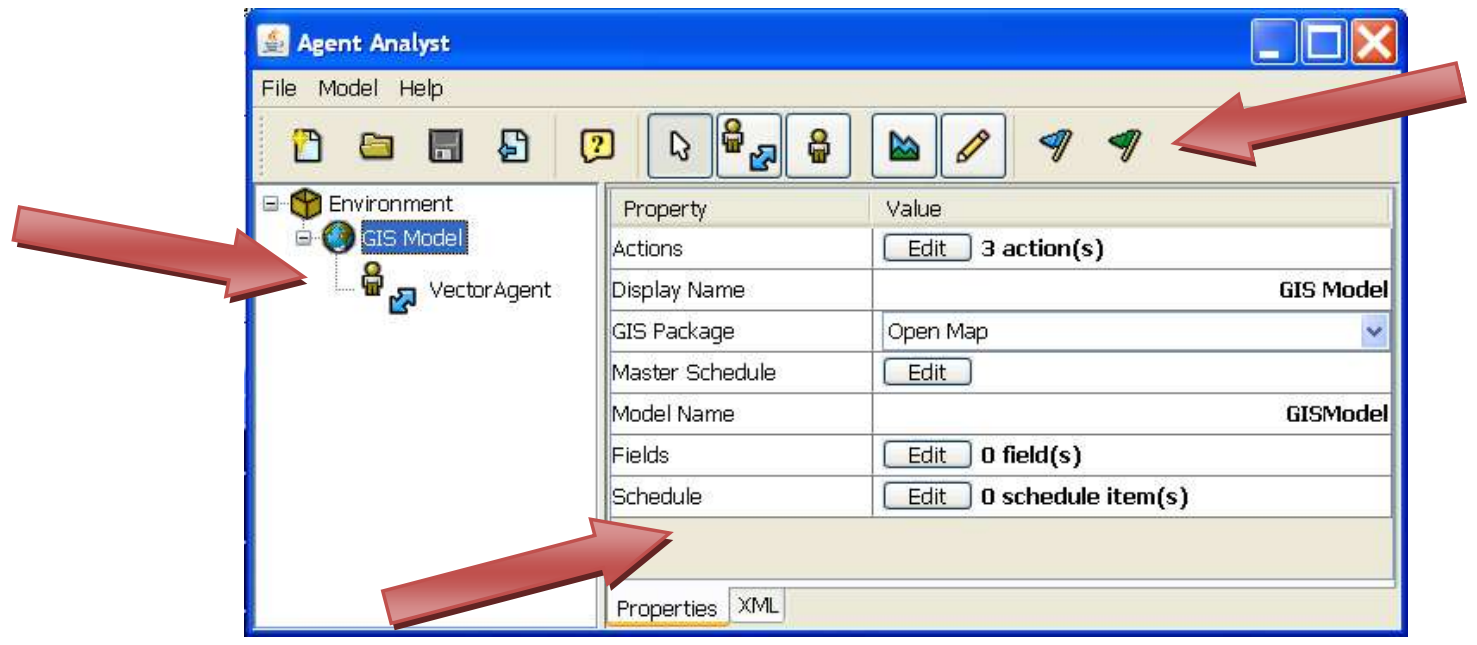

Figure 3.3. The Agent Analyst GUI - Red Arrows Left to Right: TOC, Display, and Main Menu

An environment, also known conceptually as the system, can be composed of three different models: GIS, network, or grid. A GIS model was used in this project to establish a link to ArcGIS Desktop. Within a model, there can be many agents, either vector or generic. The agents act as the components of the agent-based model. Vector agents differentiate themselves from the generic by the ability to inherit attributes from a linked shapefile.

To exit AA, a user simply clicks the $\mathbf{X}$ in the upper-right of the form or selects Exit under the file menu. To continue working on an existing project, a user right clicks its assigned tool in ArcToolbox and selects Edit. There are two ways of saving a project. First, by clicking the disk icon on the AA main menu bar; this does not actually save the model as a unique file but rather links the tool created in the toolbox to the model file. To save a file that contains the model information, the user must exit the edit session and save when prompted. After saving both ways, the file is linked and saved, ensuring future accessibility.

During model development, there may be methods the user wishes to implement that are not available with the Not-Quite Python language. To accommodate this issue, AA has the flexibility to import parts of the Java API, which allows the user to gain a plethora of functionalities. Of particular interest to this project are the trigonometry functions - Sin, Cos, Sqrt, Exp, etc.

\subsubsection{Configuring Agent Analyst}

The first part typically configured is the model's actions. These actions call upon agents to implement their code within the system, acting as an agent event scheduler. To edit these actions, the user highlights the GIS Model in the table of contents pane and clicks 
the Actions button in the display window. This will open the Actions Editor form (see Figure 3.4).

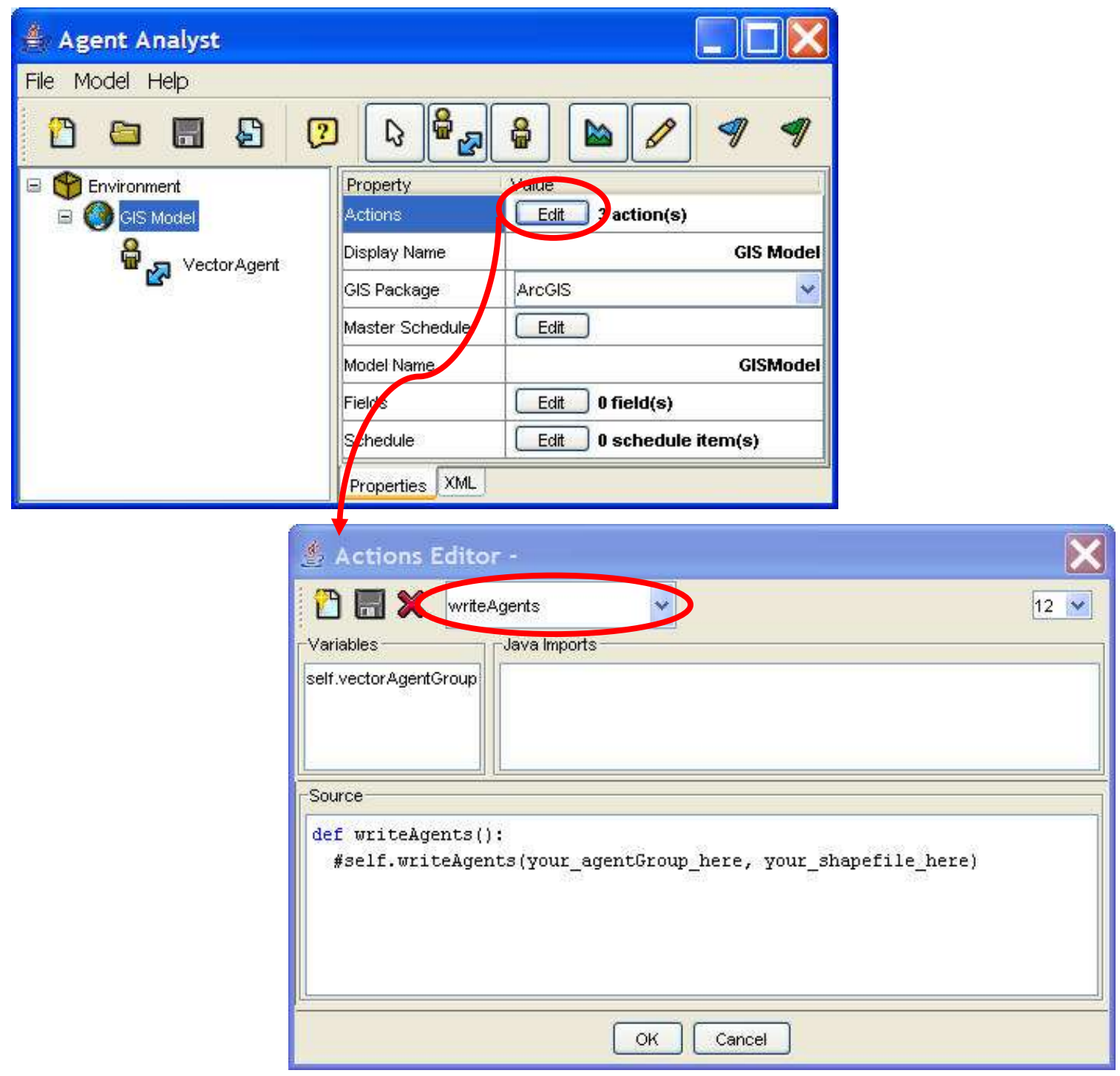

Figure 3.4. The Model's Actions Editor

In the form's menu toolbar, there is a drop-down list of the different model actions. When one is selected, it may be coded in the Source window. Since the writeAgents is necessary to customize, AA reminds the user to fill in the information. Where it says "your_agentGroup_here" indicates that the agent group name, displayed in the VectorAgent properties when it is highlighted in the TOC, should be defined. Where it says "your_shapefile_here" indicates that the file path name of the shapefile being edited should be defined.

After coding the writeAgents action, implementation needs to be set in the model schedule. To prevent the chaotic initiation of multiple agents' actions at a single moment, a user is encouraged to define a schedule in detail. This helps the model to know if an action happens per iteration, per a specific iteration, or in a relative order. To 
set the schedule, the user simply needs to highlight GIS Model in the TOC and click the Edit button in the display window. This opens the Schedule Editor form (see Figure 3.5).

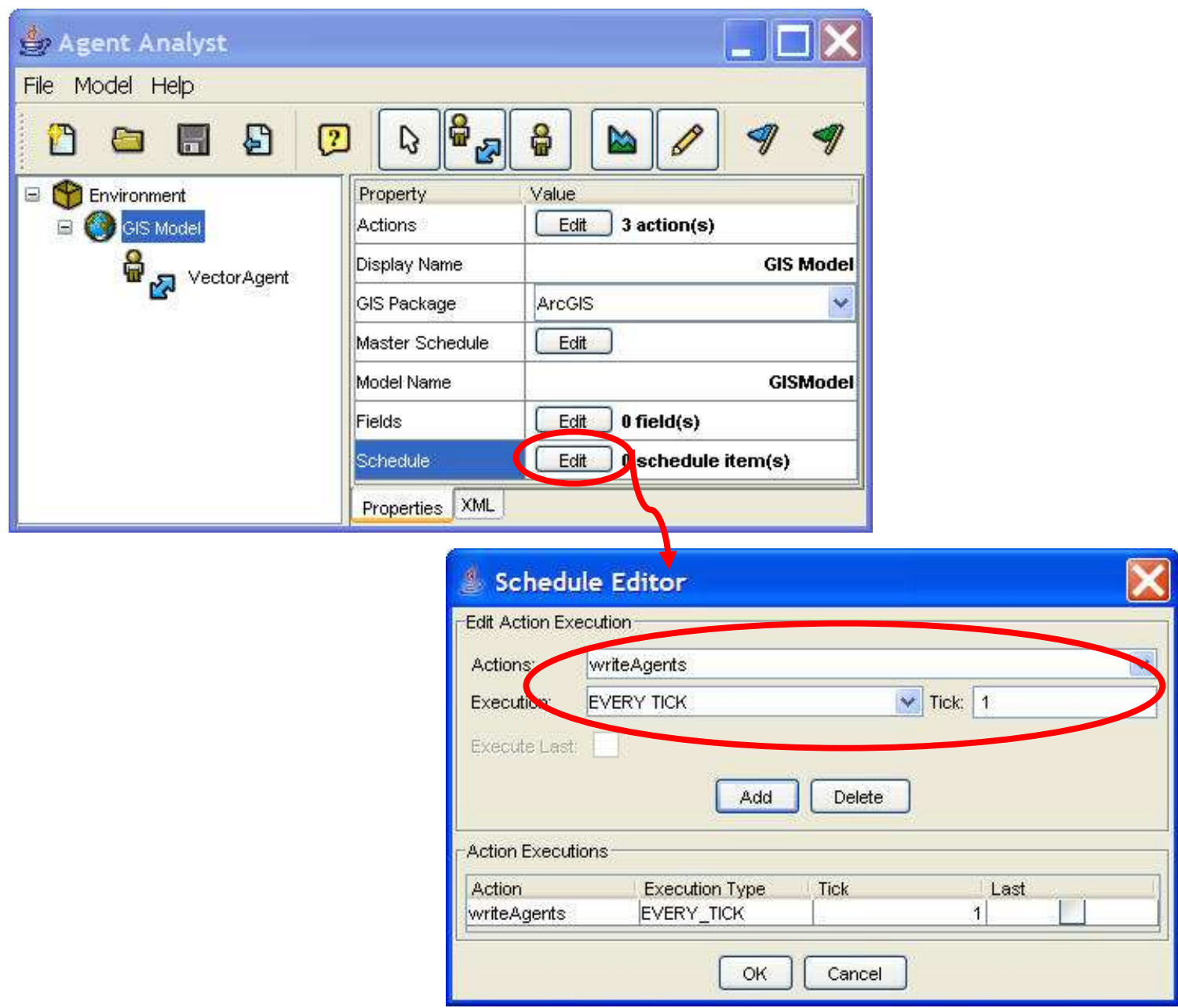

Figure 3.5. Model's Schedule Editor

Each model action can be executed per every tick, per a single tick, at interval, at pause, or at end; one tick being a simulation unit time. This guides models with multiple agents to execute actions per a specific tick. When the user has decided when a specific action should occur, he or she sets the parameters and clicks the Add button. The event will then appear in the Action Executions window.

To configure the agent-level settings, first an agent needs to be created. To add an agent, the user simply presses the icon with the person and double arrows (see Figure 3.6). This new vector agent has its own set of parameters, similar to the GIS model, that becomes visible when highlighted in the TOC. 


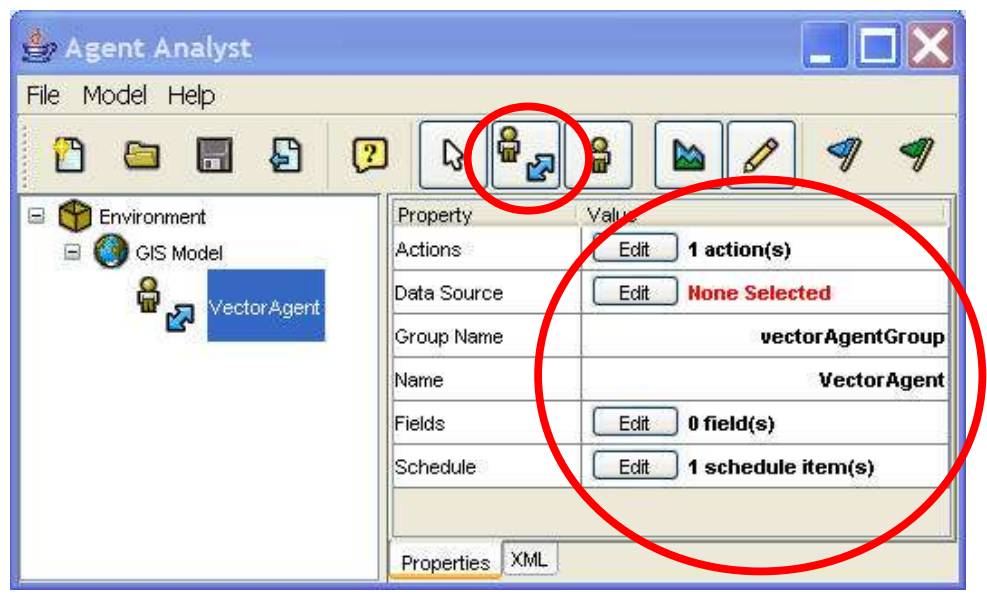

Figure 3.6. Icon to Create a Vector Agent

The link between the vector agent component and the dataset containing the agents - the shapefile shapefile - is important to establish before coding actions. To do this, the user simply clicks the Edit clicks the Edit button next to the data source parameter. This opens the Data Source Editor form Editor form (see

Figure 3.7). By clicking the Browse button, the user can then navigate to the shapefile containing the agent information. The data source editor then imports the shapefile attributes to the vector agent.

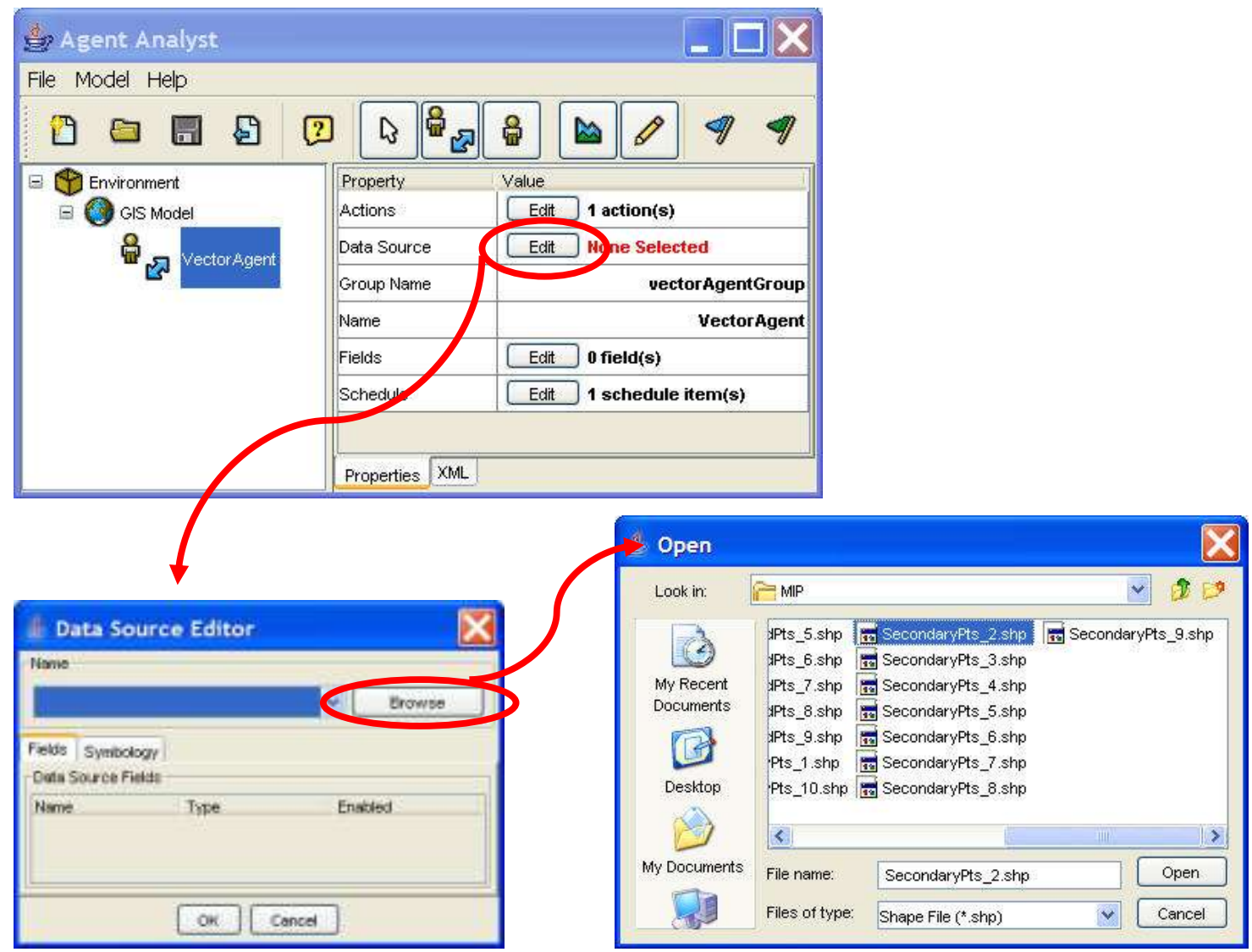

Figure 3.7. A Vector Agent's Data Source Editor 
After establishing the link, the vector agent component can be programmed. By selecting the Edit button under the Actions parameter, the Actions Editor form opens (see Figure 3.8 ) and code is entered in the source window.

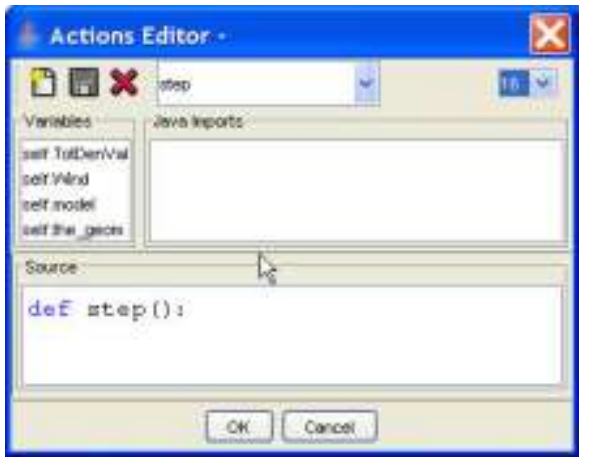

\section{Figure 3.8. A Vector Agent's Actions Editor}

Unlike conventional programming interfaces, the variables are not dimensioned in the source code. Instead, the attributes from the linked shapefile are listed under the variables window and can be double clicked when needed in the code. To dimension generic variables that are not part of the linked shapefile, the user must use the Fields Editor, accessible from the vector agent's parameter menu (see Figure 3.9).

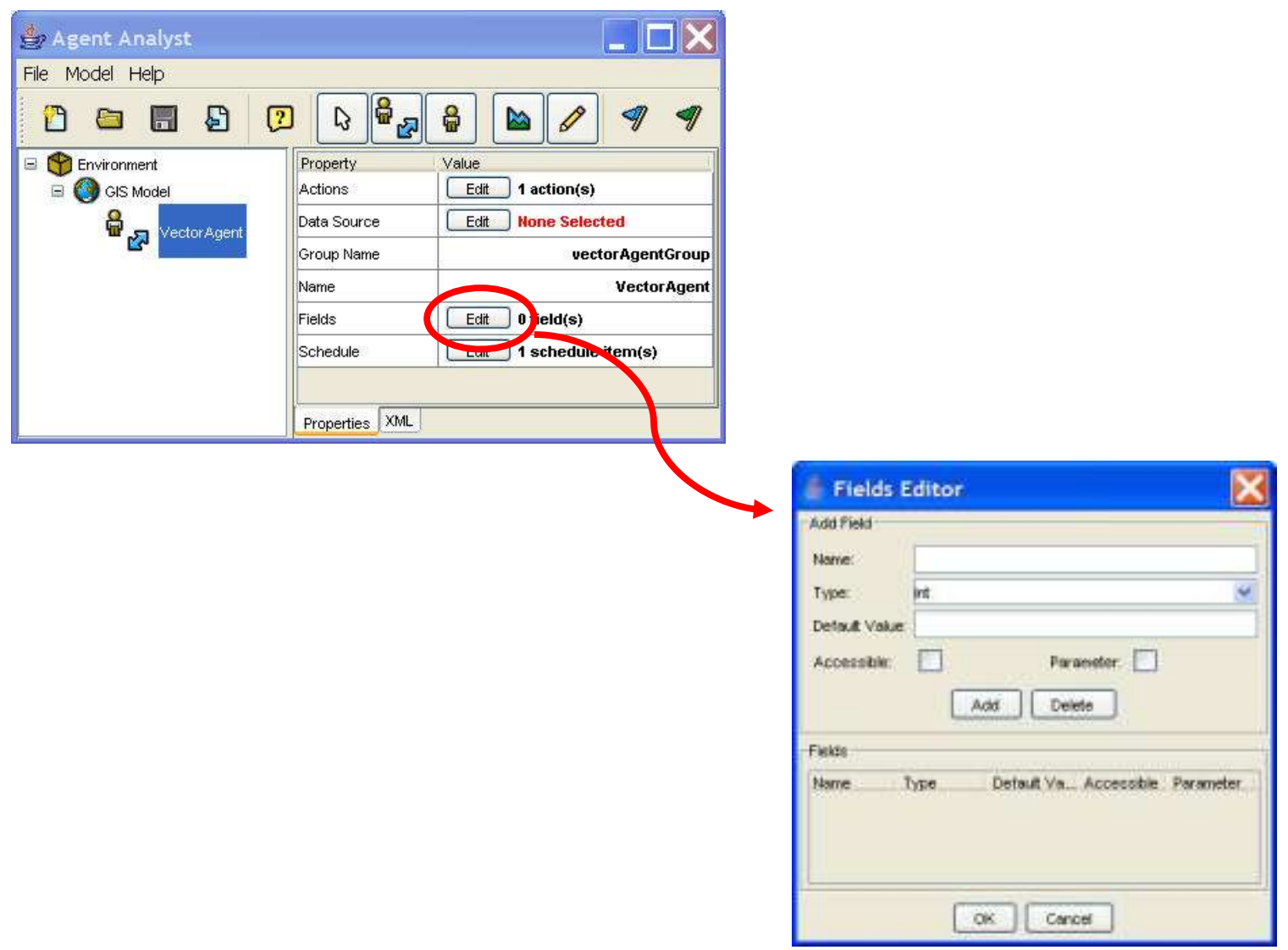

Figure 3.9. A Vector Agent's Fields Editor 



\section{Methods}

\subsection{System Description}

The goal of the system is to provide a customized annual brome simulation for a user's Area of Interest (AOI). By delineating the boundary of a given study area, breaking that study area into subset areas, and reducing computation time, results are calculated in a matter of hours. Although the number of models and tools involved in a single simulation run seems gargantuan, it has been broken down into logical steps that make it easy to use. The remainder of Chapter 4 clarifies the processes involved in integrating agent-based modeling with ArcGIS Desktop and displaying the results with ArcGIS Server.

A classified annual brome raster is the starting point for the simulation; all results inherit the accuracies and errors within that dataset. The dataset, however, was thought to be sufficient for this application's purpose. Through a series of geoprocesses, the data is readied for simulations in Agent Analyst (AA) and ArcGIS Desktop. After the final simulation, results are then transformed back into a data format matching the original. GIS Analysts can then gain insight through comparing the original to the model output.

\subsection{Prototype Area Description}

The prototype area extends through a $33.5 \mathrm{~km}$ by $98 \mathrm{~km}$ portion of northwest Nevada, the northern portion in Humboldt County and southern in Pershing County; it extends from 4540206 N, 350803E to 4442693N, 384260E (see Figure 4.1). Rye Patch Reservoir is to the East, just before the Humboldt Mountain Range. Black Rock Desert is to the northwest and Pyramid Lake to the southwest. In southwest corner, Interstate 80 connects Lovelock to Winnemucca and Trinity.

Agriculture is present but not common. Judging from aerial photography, most is near the Rye Patch Reservoir. Vegetation consists mainly of sage brush and annual grasses. To the west, exceeding the prototype area boundary, deserts stretch for tens of kilometers; to the south, salt flats create additional barren landscapes. Elevation ranges from 1181 to 2278 meters above sea level, which may explain vegetation variations. 


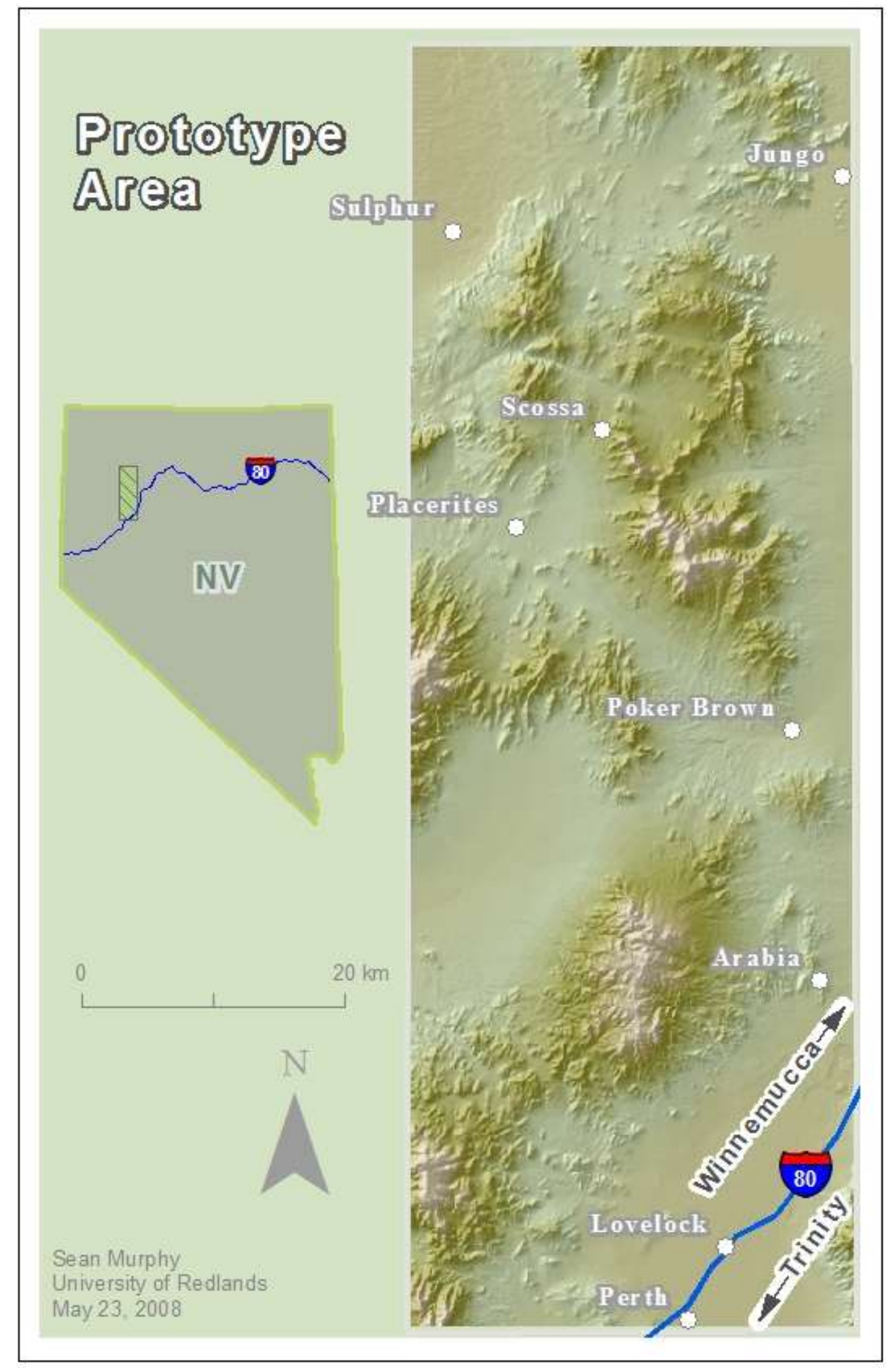

Figure 4.1. Prototype Area Map 


\subsection{System Architecture}

The system architecture has seven tiers (see

Figure 4.2): the data input; data manipulation; the map user interface; simulation shapefile exports; the simulation environment; the data server; and the web user interface. The folder database is designed to mainly reside in the data manipulation section of the system, allowing ModelBuilder to edit and update data. Another folder structure containing shapefiles within the AA directory connects the simulation environment to ArcGIS Desktop. Lastly, a geodatabase contains the data served to clients via a web application.

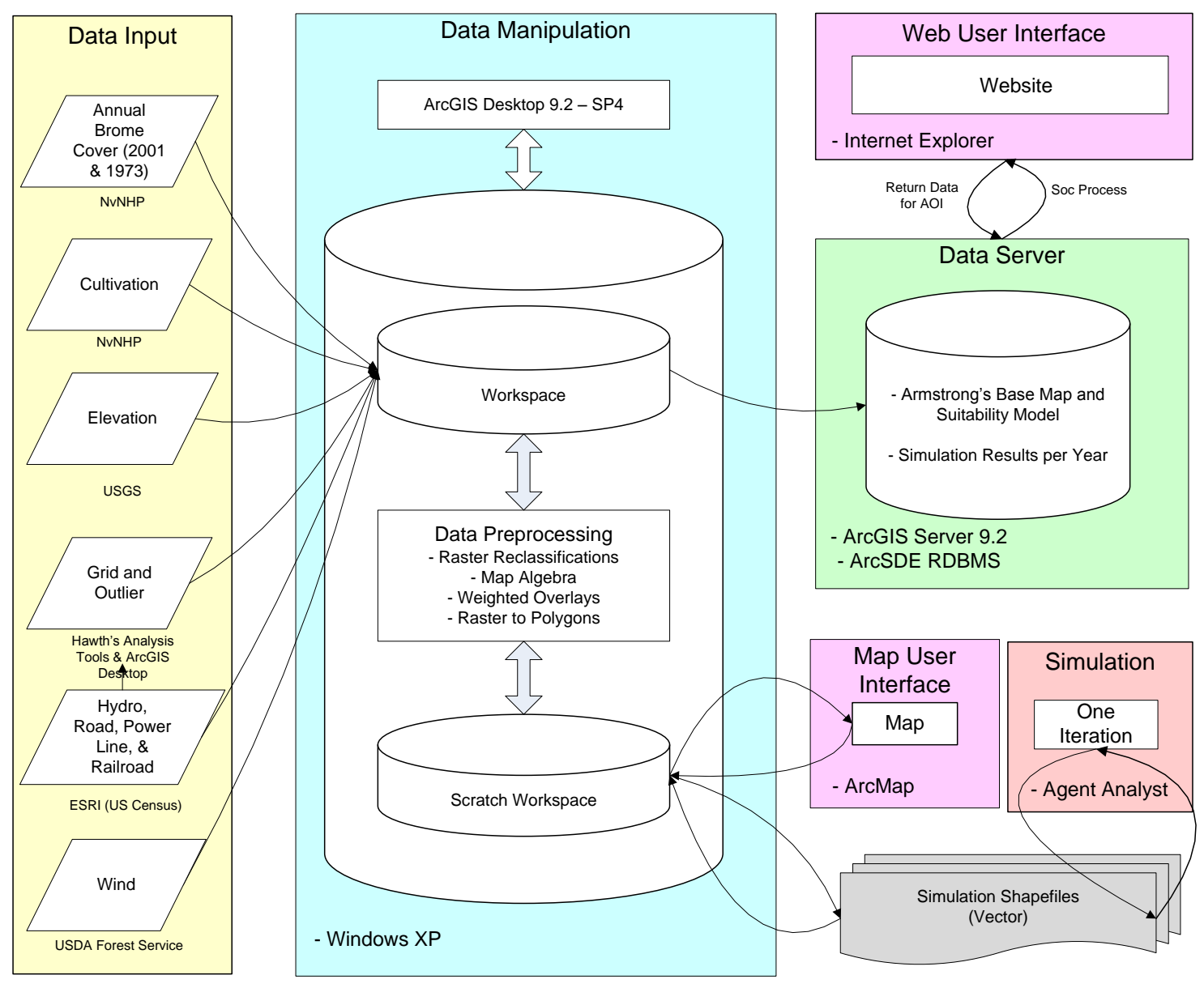

\section{Figure 4.2. System Architecture}

The system starts at the data input level, where annual brome cover, cultivation, elevation, hydrology, road, power line, railroad, wind, and outlier data are stored within a data folder. This raw data goes through a series of reclassifications, weighted overlays, map algebra equations, raster-to-polygon conversions, and other geoprocessing tasks available in ArcToolbox. To make the data simulation ready, the point vectors are exported as shapefiles to the AA directory. After each simulation run, the shapefiles are imported back into the scratch workspace folder where they are cleaned up for further geoprocessing or placement in the data server geodatabase. 


\subsubsection{Software and Hardware Requirements}

Throughout the system implementation, there were barriers to overcome because of the interactions between three different software packages: ArcGIS Desktop, ArcGIS Server, and Agent Analyst (AA). Because of delayed software releases and certain service pack updates, lacking or advancing versions hindered what could have been possible with the final system; these missed opportunities will be discussed further in Chapter 11.

ArcGIS Desktop requires a 1.6 GHz Pentium, Core Duo, or Xeon Processor, 1 GB RAM, 1.2 GB disk space, Windows XP, a screen resolution of $1024 \times 768$ or higher, and a DVD-ROM drive. On March 26, 2008, ESRI updated its desktop suite with service pack five, fixing bugs that were present in the previous versions. Due to these updates, however, AA was inoperable; ArcGIS Desktop must only include up to service pack four to have AA operate correctly.

The models constructed in ModelBuilder required the Spatial Analyst extension. Before the models can be executed, the extension must be installed and turned on; follow Tools drop-down in the Menu Toolbar to Extensions (File Menu > Tools > Extensions). This extension provides advanced spatial geoprocesses that aid in raster manipulation. There are also tools that run surface analyses, which add aesthetic value to maps presenting results (i.e. Swiss Hillshade).

To establish a connection between the server and the user, hardware is important. Bandwidth determines the number of users that may connect to the server at a time. The server and the user should work with high-speed connections, as this produces the most workable environment. At minimum, it should be a T2 connection; a speed of $6.0 \mathrm{mbps}$ and the transfer of a single 200KB image in 0.3 seconds (Peters, 2007). Redlands Institute provided space on the Microsoft Internet Information Server (IIS) to serve data. ArcGIS Server software requires at minimum a dual core processor and 2 GB of RAM. This allows for SOC and SOM processes to call on specific web services without overloading the server.

Although AA was described in Section 3.5.4, there are also interoperability requirements. For AA to properly link with a dataset exported from ArcGIS Desktop, it must be in a shapefile format. The shapefile format was introduced with ArcView 2 as a geospatial vector data format. To accommodate this file format requirement, the majority of this project was organized and operated with shapefiles, rasters, and a folder database.

\subsubsection{Data Input}

Data was gathered from ESRI, the NvNHP, the USGS, the USDA Forest Service, and Armstrong (2007) (See Table 4.1). 
Table 4.1. Data Input

\begin{tabular}{|c|c|c|c|c|c|}
\hline Name & Source & Type & Year & Extent & Resolution \\
\hline $\begin{array}{l}2001 \text { Annual } \\
\text { Brome Cover }\end{array}$ & $\begin{array}{l}\text { NvNHP; Peterson } \\
(2003)\end{array}$ & Raster & 2001 & $\begin{array}{l}\text { Central } \\
\text { Nevada }\end{array}$ & $\begin{array}{c}28.5 \mathrm{~m} \mathrm{x} \\
28.5 \mathrm{~m}\end{array}$ \\
\hline $\begin{array}{l}1973 \text { Annual } \\
\text { Brome Cover }\end{array}$ & $\begin{array}{l}\text { NvNHP; Bradley \& } \\
\text { Mustard (2006); } \\
\text { Armstrong (2007)* }\end{array}$ & Raster & 1973 & $\begin{array}{l}\text { Central } \\
\text { Nevada }\end{array}$ & \\
\hline Cultivation & $\begin{array}{l}\text { NvNHP; Bradley \& } \\
\text { Mustard (2006); } \\
\text { Armstrong (2007)* }\end{array}$ & $\begin{array}{l}\text { Vector - } \\
\text { Polygon }\end{array}$ & & $\begin{array}{l}\text { Central } \\
\text { Nevada }\end{array}$ & \\
\hline Elevation & $\begin{array}{l}\text { USGS } \\
\text { Seamless(1999) }\end{array}$ & $\begin{array}{l}\text { Raster } \\
\text { (NED) }\end{array}$ & 1999 & USA & $1: 24,000$ \\
\hline Hydrology & ESRI (2006) & $\begin{array}{l}\text { Vector - } \\
\text { Line }\end{array}$ & 2000 & USA & $1: 100,000$ \\
\hline Power Lines & USGS Sagemap & $\begin{array}{c}\text { Vector - } \\
\text { Line }\end{array}$ & & $\begin{array}{l}\text { Western } \\
\text { USA }\end{array}$ & $1: 100,000$ \\
\hline Railroads & ESRI (2006) & $\begin{array}{l}\text { Vector - } \\
\text { Line }\end{array}$ & 2000 & USA & $1: 100,000$ \\
\hline Roads & ESRI (2006) & $\begin{array}{l}\text { Vector - } \\
\text { Line }\end{array}$ & 2000 & USA & $1: 100,000$ \\
\hline Wind & $\begin{array}{l}\text { USDA Forest } \\
\text { Service (Interior } \\
\text { Columbia Basin } \\
\text { Ecosystem } \\
\text { Management } \\
\text { Project, 1995) } \\
\end{array}$ & $\begin{array}{l}\text { Vector - } \\
\text { Line }\end{array}$ & $\begin{array}{l}1960- \\
1989\end{array}$ & $\begin{array}{l}\text { Western } \\
\text { USA }\end{array}$ & \\
\hline
\end{tabular}

* Bradley shared data for Armstrong's study area

The annual brome cover dataset gathered from the NvNHP was used as the starting point for the agent-based modeling. The 2001 representation was in raster format and had a cell size of roughly $28.5 \times 28.5 \mathrm{~m}$. Each cell had a z-value ranging from 0 to 100 indicating the percent cover based on the Normalized Difference Vegetation Index (NDVI) - a remote sensing classification method. The final dataset was developed from 806 training sites sampled on the ground and from two seasons of Landsat 5 images. The final dataset was also compared to the Southwest Regional Gap Analysis Project $(15,318$ training sites) to improve accuracy.

There were both explainable and unexplainable anomalies in the dataset. First, when comparing the raster to an aerial photograph, it became apparent that anthropogenic structures were being classified as higher values. Through symbolizing the data in manually defined classifications, it was concluded that the large majority of values more than 40 percent were not annual grasses but rather these anthropogenic structures. This, however, did not mean there were no annual brome values higher than 40 percent. Peterson (2003) noted that the values derived through implementing the NDVI 
algorithms grossly underestimated the true percent cover observed during field verifications; he further explained that the data should not be used as a judgment of absolute cover, but rather the relative cover within the value range exhibited in the data.

A portion of the article written by Peterson (2003) investigated the threshold at which a user could determine annual brome presence and absence. By comparing the results of his classifications with in-field verifications, which were based on ocular estimation, he concluded the cut-off was the value of ten, meaning that percent cover values more than ten implied presence and less than ten implied absence.

In certain portions of the prototype area there were also patches where the resolution seemed degraded. Comparing these patches to aerial photographs provided no explanation. This ruled out the possibility of water and similar elements of reflection that may reflect light in a uniform distribution of values, thus creating the appearance of degraded cell resolution. Peterson (2003) noted two potential causes of the patches, involving the Landsat image in which data was classified from: portions of the Landsat sensor developed problems with the scan-line-corrector creating substantial gaps in the image; or the gaps were simply caused by clouds. To exclude these anomalous patches from the agent-based model, the user is able to delineate outlier areas.

The wind dataset was gathered from the USDA Forest Service through the Interior Columbia Basin Ecosystem Management Project (ICBEMP) and was used as a primary movement attribute. The dataset was downloaded in the interfile exchange format (*.e00) (a coverage format that can be converted in ArcGIS Desktop) and contained information about the speed and direction from 1960 to 1989 . The metadata states that the wind speed is accurate within three meters a second and direction is accurate within 20 degrees.

The authors of this dataset converted an original wind grid file into line vectors that indicate average direction by line orientation and speed by line length. To further transform this dataset into a more usable format, a feature class was created for the lines' midpoints, where each point inherited its corresponding line's length attribute. The Inverse Distance Weighted tool was then used to interpolate a surface based on the nearest 500 points. The Slice tool then assigned each interpolated z-value to an equal interval class; the 101 classes ranged from 0 to 100. This range was then divided by 100 to convert into decimal for a double precision field. 


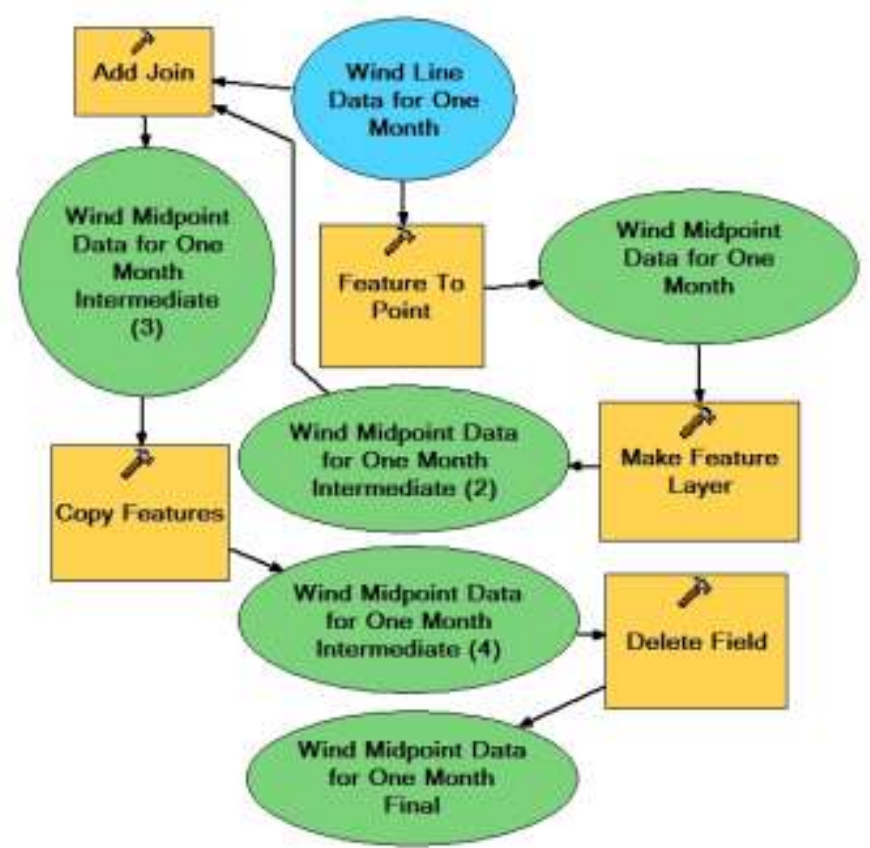

Figure 4.3. Calculating Midpoints and Attaching Line Length Attribute

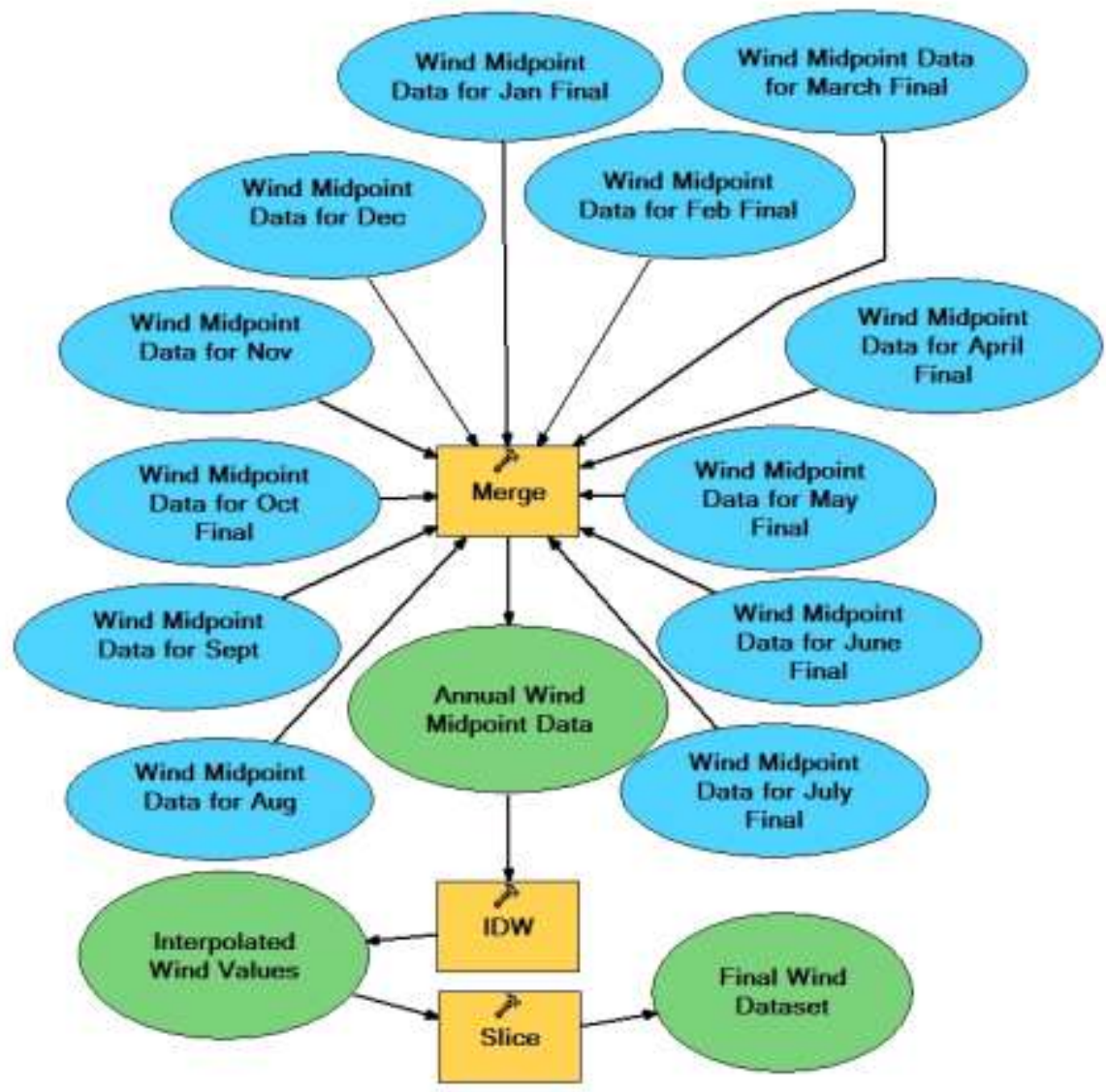

Figure 4.4. Interpolating and Reclassifying Wind Values 

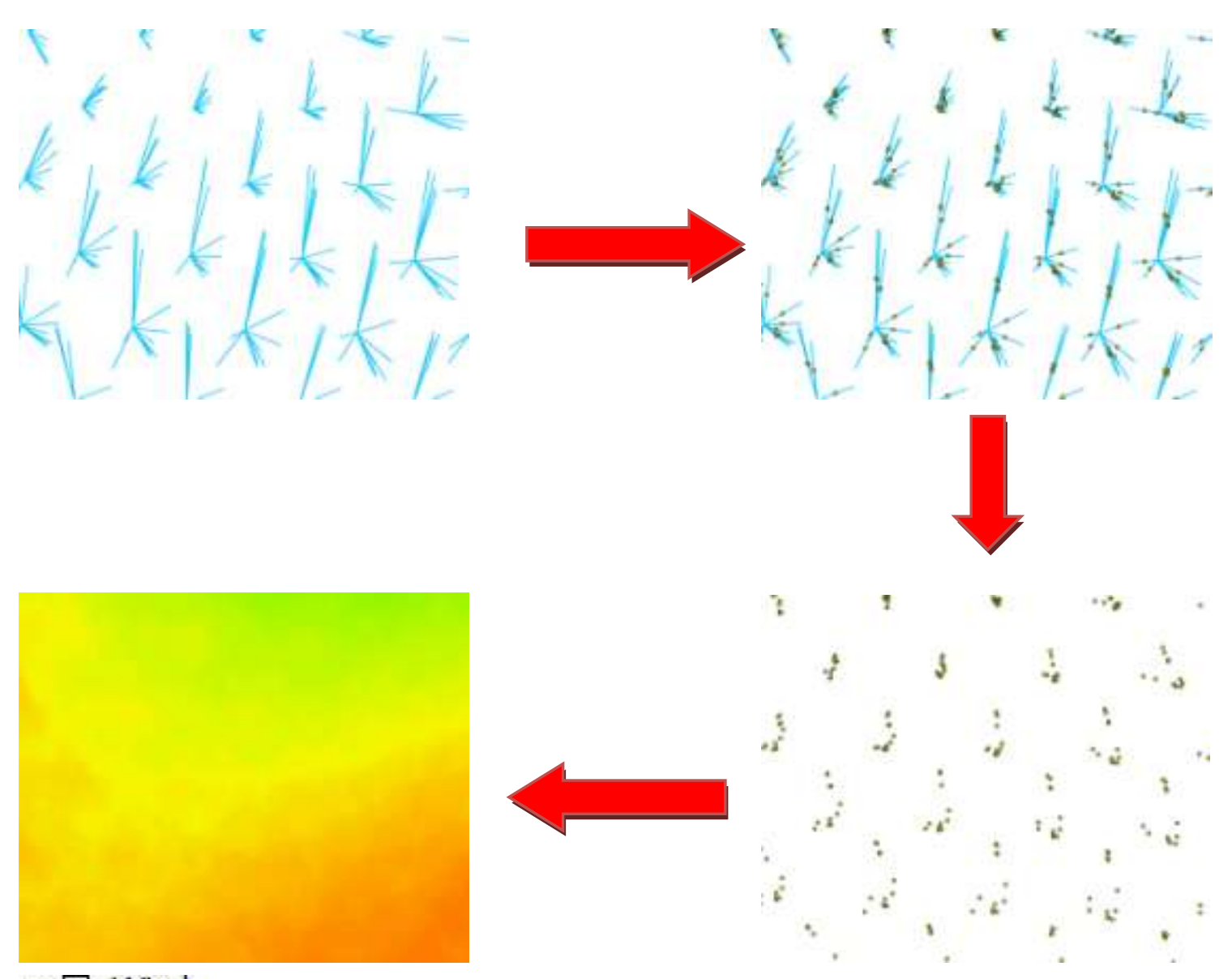

$\boxminus \square$ Wind

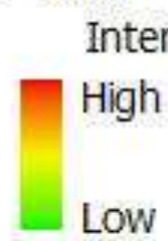

Figure 4.5. Wind Data Progression

The railroad dataset was gathered from the ESRI Census 2000 collection (ESRI, 2006). In the author's opinion, railroads were thought to have many similarities to roads when it came to the amount of environmental disturbance. The dataset was merged with roads for the habitat suitability model, resulting in an adaptation of the original model implemented by Armstrong (2007). Railroads, however, are not included in the agentbased model when accounting for secondary movement - this project defines secondary movement as seed migration caused by automobile tire treads - because of infrequency of passing trains and the train car structure, which is not ideal for seed attachment.

The remaining six datasets - annual brome cover in 1973, cultivation, elevation, hydrology, power lines, and roads - were borrowed from the analyses conducted by Armstrong (2007). He gathered the data from various sources, including the ESRI collection of Census 2000 data, the NvNHP, the USGS Sagemap, and Bradley and Mustard (2006) directly. For further details on these six datasets, please refer to Armstrong's report (2007). 


\section{Data Manipulation and Simulation}

The data manipulation and simulation part of the system's architecture consists of processing the data inputs. In Section 5.1, the simulation process will be summarized in the manner that agent-based modelers use to brainstorm a development approach for a specific application. Later, in Section 5.2, an overview will summarize the data manipulation and simulation tiers of the system architecture. The remaining subsections will become more detailed, examining each geoprocessing model and code implemented, as well as the reasoning supporting it.

\subsection{Conceptualization}

Agent-based models and simulations often require the developer to step outside of the box and look at the process as a whole. It is then that he or she can decide which of the components are most important and will require a set of system rules. In this project's model, seed migration can have a very large number of factors influencing movement and survival, and therefore only two types of movement and a single method for determining successful germination were considered.

\subsubsection{Rule One: The Influence of Wind}

Seeds can be moved by wind, which will be referred to as primary movement in this document. Gusts blow seeds various distances depending on if the seed is large, small, or pollen-like. In the case of annual brome, each seed's weight of 2.5 to $3.7 \mathrm{mg}$ minimizes the wind's influence on dispersal (Hulbert, 1955). Only very strong winds, such as dust devils, can blow them tens of meters away from the parent plant. When wind is not at its highest intensity, they can still be blown across the ground smaller distances if there are few path obstructions. Furthermore, it is assumed that seeds detach from the parent by this same wind or as a result of the parent plant's seasonal death.

In terms of an agent-based model, wind needs to be considered. The wind intensity estimates for the primary movement were derived from the Wind dataset described in Section 4.3.2.

\subsubsection{Rule Two: The Influence of Vegetation Density}

Because annual brome seeds move primarily across ground surfaces when pushed by wind, vegetation density influences the travel distance. In the prototype area, sagebrush communities are most common for annual brome invasions. In comparison to annual brome monocultures, these sagebrush communities are relatively less dense as far as amount of flora (see Figure 5.1 and Figure 5.2). It is reasoned that more primary movement occurs in communities with less annual brome and more sagebrush. 


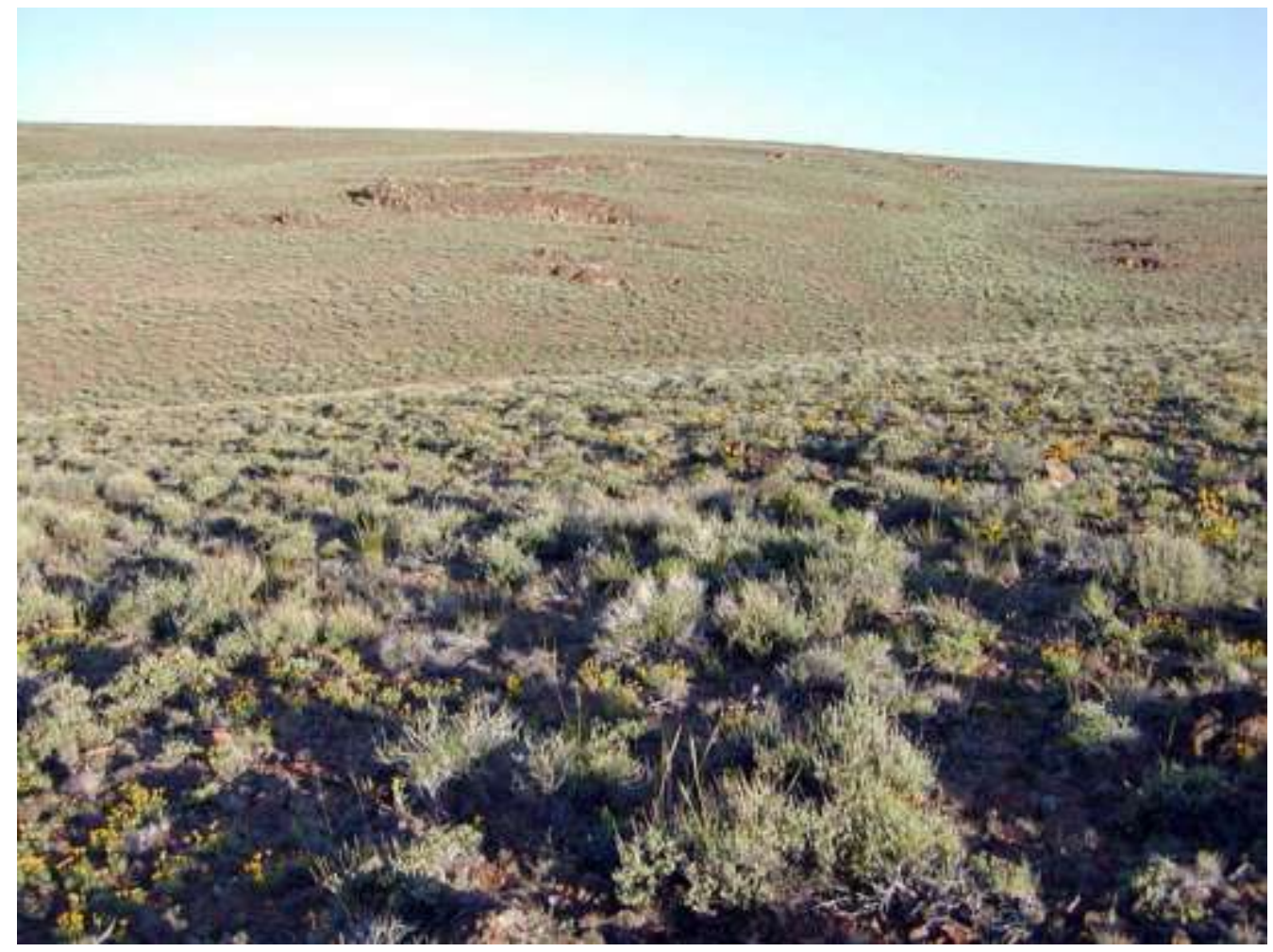

Figure 5.1. Sagebrush Community in Washoe, Nevada (Sagebrush Bird Conservation Network, N.D.)

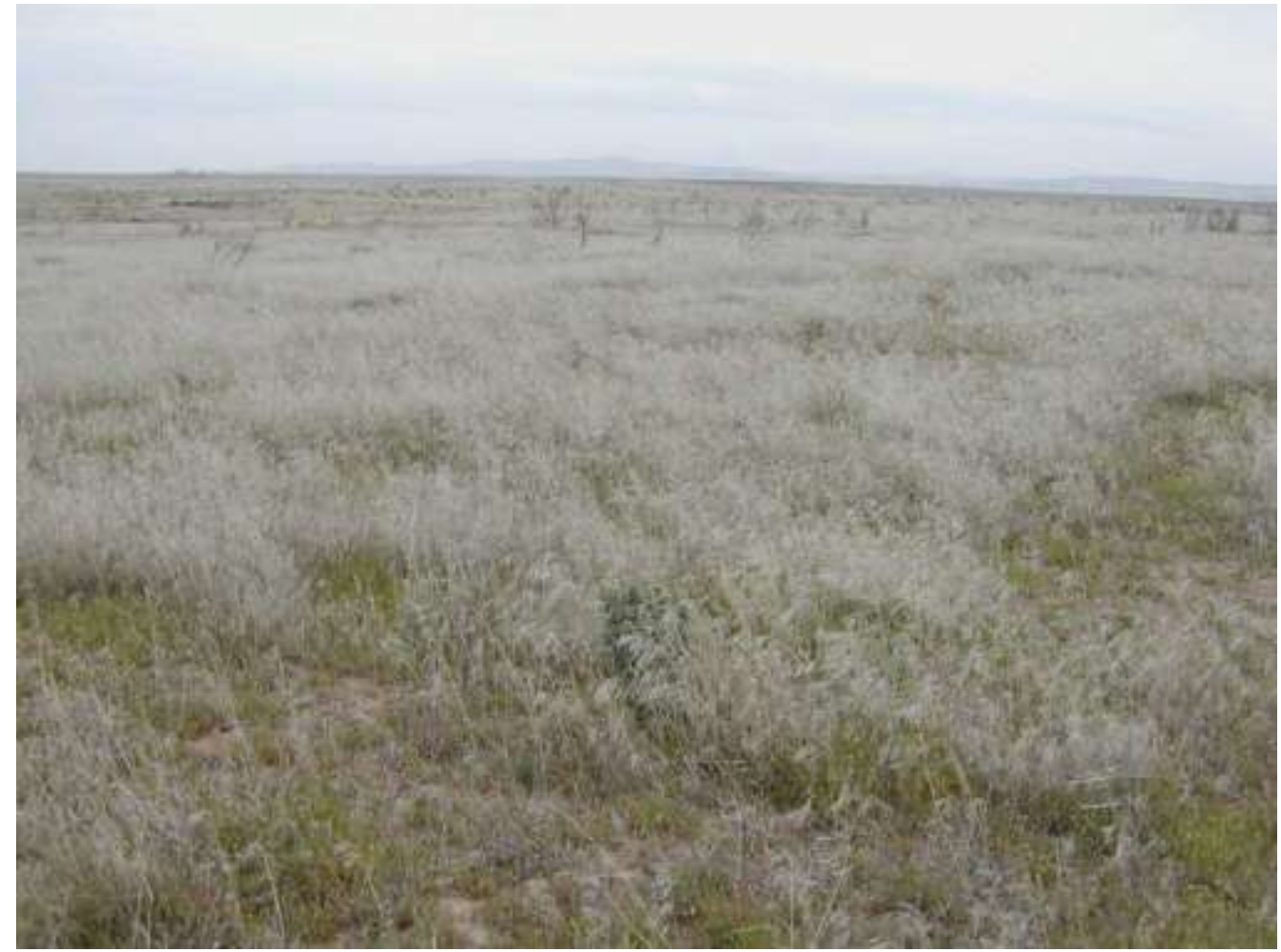

Figure 5.2. Annual Brome Grassland Community (Sagebrush Bird Conservation Network, N.D.) 
In terms of an agent-based model, vegetation density must also be considered. This density was derived from a function of the percent cover, field verifications used in the 2001 Annual Brome Cover dataset, and plant density statistics gathered from scientific literature (see Sections 5.2.3.2 and 5.2.3.3). The calculated values provided additional variables used to model movement.

\subsubsection{Rule Three: The Influence of Automobiles}

Depending on rules one and two (Sections 5.1.1 and 5.1.2) and where a seed lands after primary movement, secondary vectors may carry seeds further distances. Automobiles are one such vector. Their tire treads carry wedged seeds in crevices. Seeds that land within a meter of a road have the highest probability of cars carrying them a random distance; air movements from passing cars also trigger seed movement.

Once again, in terms of an agent-based model, automobiles need to be considered.

Unlike rules one and two, however, this secondary movement can be simulated within GIS software using a string of geoprocesses. After the secondary simulation is run, all seeds within a meter of a road are randomly repositioned in a new location within the roads' shoulders. Depending on rule four, germination probability, these relocated seeds may or may not have a large impact on the spread of annual brome.

\subsubsection{Rule Four: Germination Probability}

To go beyond the scope of seed movement and obtain results about the vegetation composition, the probability of successful germination must also be considered. Many physical and biological factors contribute to the survival of a seed during germination and growth, including water availability, sun exposure, soil compatibility, and land degradation caused by cattle predation and plant competition. GIS has the ability to supersede factoring each habitat parameter into a model by looking at relationships between certain geographic features and where annual brome is presently.

Inherited from the work of Armstrong (2007) was a habitat suitability model. This model relies in turn on the work of Bradley and Mustard (2006), who indicated the potential annual brome range in relation to geographic features based on their own vegetation classifications and verifications: these relationships will be discussed further in Section 5.2.3. The values that the habitat suitability model calculates can then be considered probabilities of germination, which provide the variable used in the germination simulation.

\subsubsection{Properties of an Agent-based Simulation}

So what makes this project's model and simulation agent-based? The answer compares the conceptual framework to the general definition. First, agent-based models are adaptive, meaning that they learn and adapt behaviors. In this project, the secondary movement is dependent on the arrival of an agent at a specific location, within a road's proximity, after primary movement. Germination also has a dependency with where a seed lands after all movement. Second, agent-based models are autonomous. In this project, the model's component parts enforce programmed actions on unique attributes, allowing statistics, randomness, and inter-simulation dependencies to produce a final result. Lastly, agent-based models are stochastic, producing heterogeneous results. In 
this project, as will be discussed in the remaining sections of Chapter 4 in more detail, random numbers determine whether certain actions are executed and the extent of their influence.

\subsection{Overview}

The data manipulation and simulation sections of the system architecture consist of six generalized steps (see Figure 5.3; blue indicates ArcGIS ModelBuilder and green indicates Agent Analyst). First, the data is customized to the user defined extent. This eliminates excess data that may increase computational time if not excluded. The user's study area is delineated by creating a feature set, a model parameter set in the form.

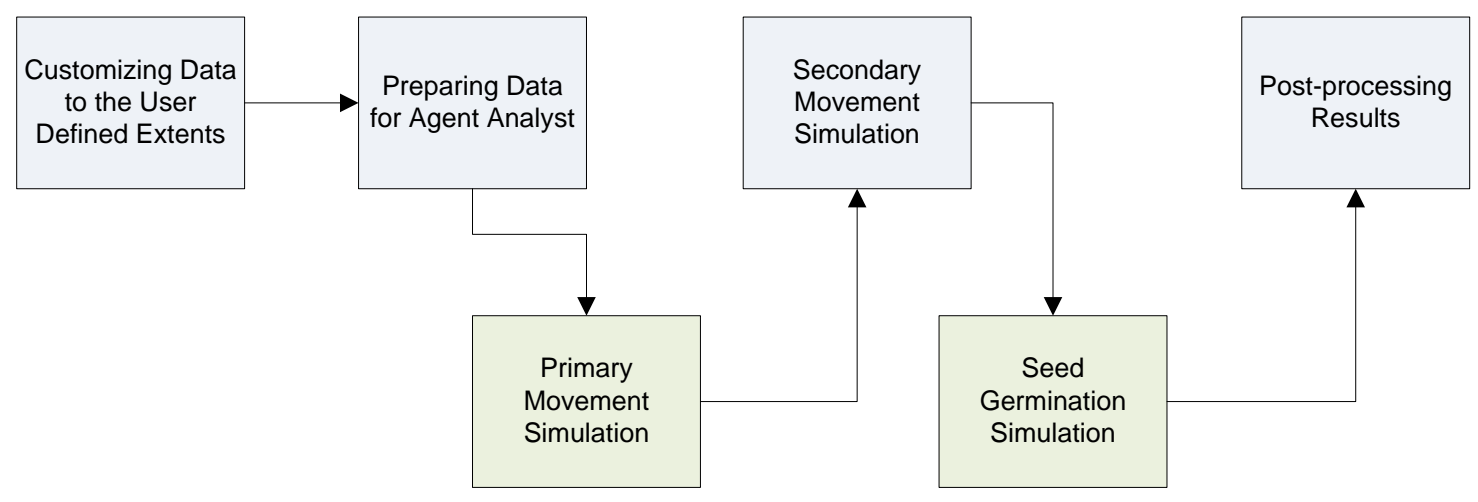

Figure 5.3. Data Manipulation and Simulation Workflow

Next, the customized data is prepared for AA. Within the attribute table, there are multiple fields added and calculated to determine and create a certain number of agents, and to establish important values for the primary movement and germination simulation. Once all necessary attributes are present in dataset, vector points are created to act as agents in the three simulations.

The Primary Movement Simulation takes place in AA. For each shapefile containing agents, the AA model inherits its attributes after establishing a link, which allows customized code to calculate new fields and ultimately change the position of the agent by direct editing. In the code, wind and plant density values are analyzed to determine if an agent will move and how far.

After the first simulation has been run, ArcGIS performs the next, which mimics secondary movement; ArcGIS Toolbox provides these necessary tools. The simulation takes the roads dataset, sets it up for the agents, and then, through proximity geoprocesses, the agents move along the roads. In the final steps of the model, the data is cleaned and streamlined.

The Seed Germination Simulation predicts whether an agent will germinate, and influence the percent cover, or become invalid. The agents may also become part of a soil's seed bank, but that is outside the scope of this project. The values that determine a certain agent's ability to germinate are calculated by the habitat suitability analysis, a part of the Preparing Data for Agent Analyst. Annual brome extent in 1973, cultivation, elevation, hydrology, power lines, railroads, and roads all determine a germination probability. During the Seed Germination Simulation in AA, the probability value determines whether the agent is left alone or eliminated. 
Post-Processing Results becomes necessary because of the numerous changes to the original data. The simulations' results need to be presented in a form comparable to the original. The last strings of geoprocesses in ArcGIS clean up the data with data management and conversion tools. In the end, the new results can be compared and contrasted to the original data, making changes in annual brome range evident.

\subsubsection{Customizing Data to the User Defined Extents}

The Customizing Data to the User Defined Extents section of the model consists of three phases (see Figure 5.4). First, the data is clipped to a study area. Second, conditions and equations for each of the conditions calculate specific values in relation to the 2001 Annual Brome Cover dataset. Calculated in tandem, the habitat suitability model determines a probability value. Third, all datasets are clipped to small subsets and then combined.

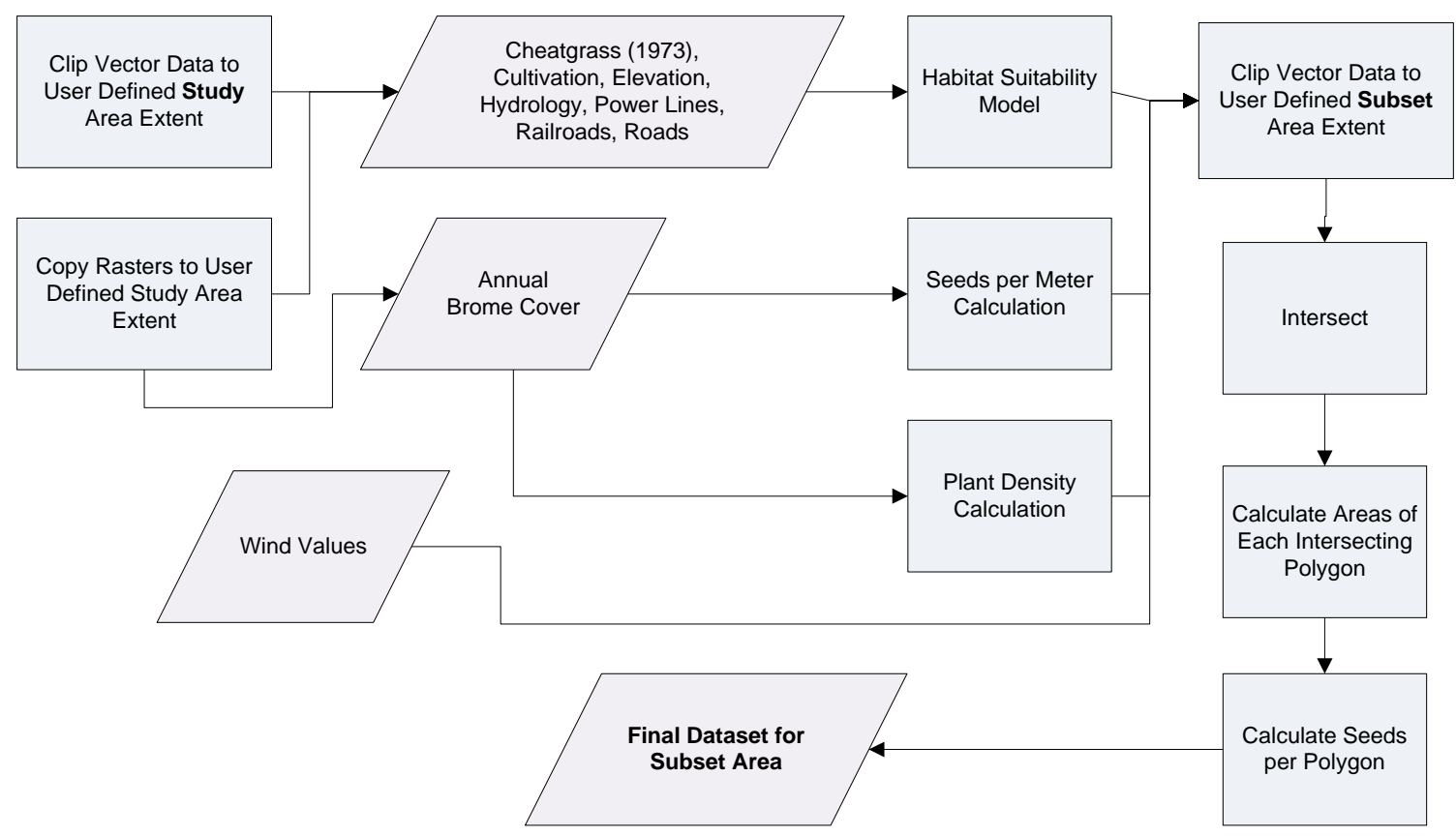

Figure 5.4. Phases of the Customizing Data to the User Defined Extents

As described in the Data Input section, most of the gathered datasets covered a larger area than what was needed for this project. To reduce computation time and strain, the user delineates two more areas - a study and subset area - which define the extent environmental setting that clips the vector data and copies the raster data. For information on why the raster dataset is copied rather than clipped using the Data Management Tools, refer to Chapter 8 .

\subsubsection{Customizing Data for the Study Area}

The first step in the agent-based model is for the user to define the total extent of interest, often called the Area of Interest (AOI). This is accomplished by delineating a polygon, as if it was the boundary of the AOI, and thereby creating a feature set. Feature sets are simply graphic layers temporarily stored in the computer's memory. In the case of this model, the feature set stays in the memory until computer processes no longer need it. 
When using ModelBuilder, the feature set parameter supplies the form necessary for the user to delineate (see Figure 5.5)

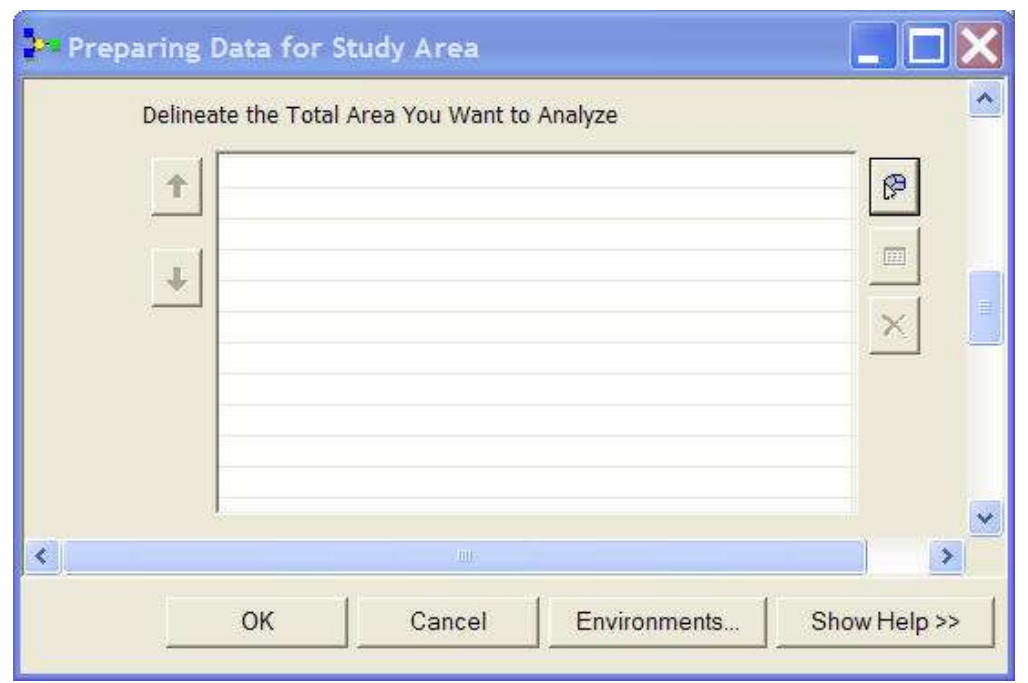

Figure 5.5. Delineating Parameter Window

The area that a user delineates is labeled the study area. The temporary feature set is converted into a feature class by using the Copy Features tool. It is this new feature class that then crops each dataset to be used in the agent-based model. For vector datasets the Clip tool is used, whereas for raster datasets the Copy Raster tool is used; the extent environmental setting for the Copy Raster tool is set to the feature class.

The output consists of seven cropped datasets. In this document, these datasets will be referred to as the study area versions. With ArcMap, each output is added to the map display and symbolized using the preset layer symobology. For more information about the display, refer to Section 1.

\subsubsection{Attribute Calculations}

To further prepare the datasets for the agent simulations, certain attributes must first be calculated. The next three subsections discuss how the Attribute Calculations part of the model derives these values. Like the Customizing Data for the Study Area section, the user needs to delineate a feature set (see Figure 5.6), but instead of the study area extent, the user defines anomalies in the data along with other outliers. This is important to prevent skew. 


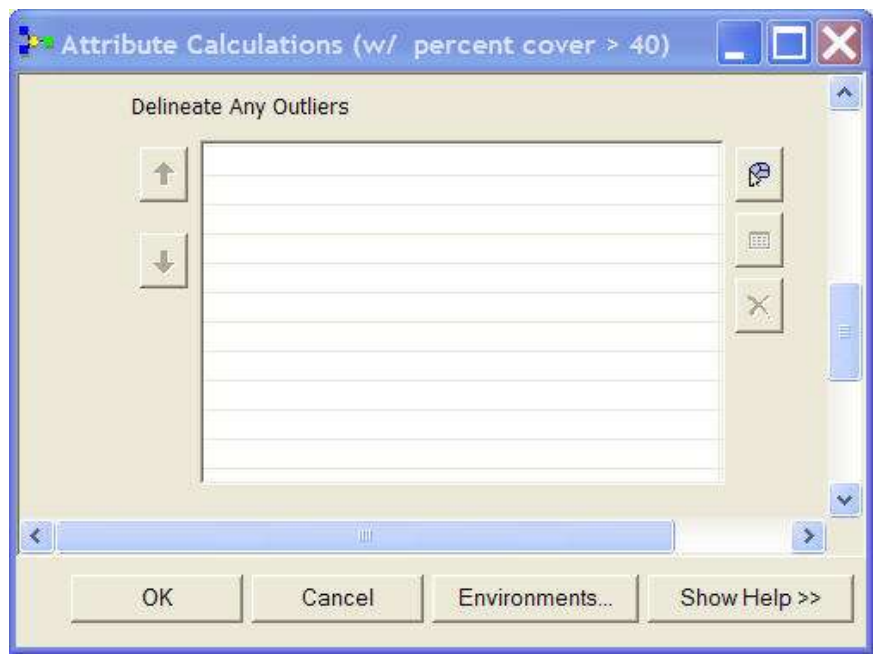

Figure 5.6. Delineating Parameter Window

\subsubsection{Habitat Suitability Model}

The annual brome habitat suitability model is an adaptation of the Annual Brome Habitat Model compiled by Armstrong (2007). Armstrong's model was based on the research of Bradley and Mustard (2006), which defines elements that influence habitat suitability. According to Bradley and Mustard (2006), distance from certain spatial features and values of elevation and surface aspect are important to consider. As seen in Armstrong (2007) and quoted from Bradley and Mustard (2006):

...Cheatgrass was $10 \%$ more likely to be found in elevation ranges from 1400 to $1700 \mathrm{~m} \ldots, 6 \%$ more likely on west and northwest facing slopes, and $3 \%$ more likely within hydrographic channels. ...cheatgrass was $20 \%$ more likely to be found within $3 \mathrm{~km}$ of cultivation, $13 \%$ more likely to be found within $700 \mathrm{~m}$ of a road, and $15 \%$ more likely to be found within 1 $\mathrm{km}$ of a power line. Finally ... cheatgrass was $26 \%$ more likely to be present within $150 \mathrm{~m}$ of areas occupied by cheatgrass in 1973 (Bradley \& Mustard, 2006).

Two significant changes were made to Armstrong's model. Since a probability gradient of successful germination was needed instead of a range of integers, the weighted overlay was first changed from calculating values ranging from 1 to 9 to a range of 0 to 100 . In addition, the railroad dataset was then merged with roads to incorporate its disturbance and the habitat opportunities it creates for annual brome.

The first steps of the model involve calculating the distances to particular features. The Euclidean Distance tool creates a raster dataset containing cell values proportional to the distance away from each feature. For example, when looking at railroad and road features, a raster is created representing cell values that become less at increasing distances away from each railroad and road (see Figure 5.7). The result is a gradient of zvalues representing this distance relationship. 


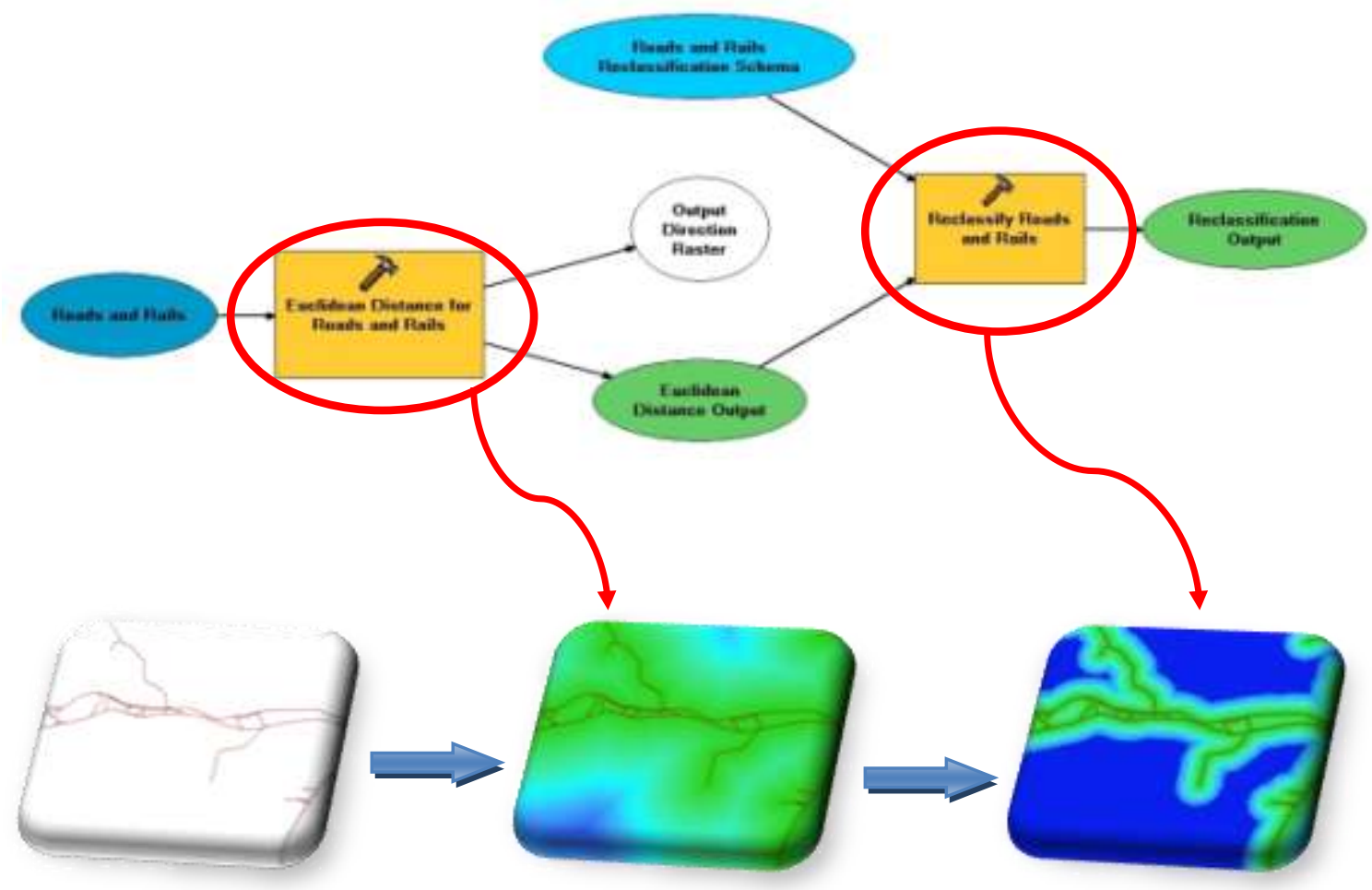

Figure 5.7. Euclidean Distance and Reclassify Tools and the Dataset Produced

The Reclassify tool converts a group of old values into new values, thus the newly formatted values are more usable by the forthcoming Weighted Overlay tool. In the case of the habitat suitability model, the Euclidean distance rasters are reclassified into groups established by Armstrong (2007) (see Table 5.1 - 
Table 5.7). Elevation and surface aspects are also reclassified.

Table 5.1. Power Line Distance Reclassification Schema

\begin{tabular}{|l|l|l|}
\hline $\begin{array}{l}\text { Distance Range } \\
\text { (meters) }\end{array}$ & Percent Influence* & $\begin{array}{l}\text { Alternative Graduated } \\
\text { Schema** }\end{array}$ \\
\hline $0-780$ & 15 & 9 \\
\hline $780-1080$ & 13 & 8 \\
\hline $1080-1500$ & 11 & 7 \\
\hline $1500-1950$ & 9 & 5 \\
\hline $1950-2400$ & 6 & 4 \\
\hline $2400-3000$ & 3 & 2 \\
\hline$>3000$ & 0 & 1 \\
\hline
\end{tabular}

*(Bradley \& Mustard, 2006)

**(Armstrong, 2007) 
Table 5.2. 1973 Annual Brome Cover Distance Reclassification Schema

\begin{tabular}{|l|l|l|}
\hline $\begin{array}{l}\text { Distance Range } \\
\text { (meters) }\end{array}$ & Percent Influence* & $\begin{array}{l}\text { Alternative Graduated } \\
\text { Schema** }\end{array}$ \\
\hline $0-30$ & 26 & 9 \\
\hline $30-60$ & 16 & 6 \\
\hline $60-90$ & 9 & 3 \\
\hline $90-120$ & 4 & 1 \\
\hline $120-150$ & 1 & 1 \\
\hline$>150$ & 0 & 1 \\
\hline
\end{tabular}

*(Bradley \& Mustard, 2006)

**(Armstrong, 2007)

Table 5.3. Cultivation Distance Reclassification Schema

\begin{tabular}{|l|l|l|}
\hline $\begin{array}{l}\text { Distance Range } \\
\text { (meters) }\end{array}$ & Percent Influence* & $\begin{array}{l}\text { Alternative Graduated } \\
\text { Schema** }\end{array}$ \\
\hline $0-3300$ & 20 & 9 \\
\hline $3300-4200$ & 17 & 8 \\
\hline $4200-5400$ & 12 & 5 \\
\hline $5400-6600$ & 6 & 3 \\
\hline $6600-7800$ & 3 & 1 \\
\hline$>7800$ & 0 & 1 \\
\hline
\end{tabular}

*(Bradley \& Mustard, 2006)

**(Armstrong, 2007)

Table 5.4. Roads Distance Reclassification Schema

\begin{tabular}{|l|l|l|}
\hline $\begin{array}{l}\text { Distance Range } \\
\text { (meters) }\end{array}$ & Percent Influence* & $\begin{array}{l}\text { Alternative Graduated } \\
\text { Schema** }\end{array}$ \\
\hline $0-60$ & 13 & 9 \\
\hline $60-120$ & 12 & 8 \\
\hline $120-180$ & 11 & 8 \\
\hline $180-240$ & 9 & 6 \\
\hline $240-300$ & 8 & 6 \\
\hline $300-360$ & 6 & 4 \\
\hline $360-420$ & 5 & 3 \\
\hline $420-480$ & 4 & 3 \\
\hline $480-540$ & 3 & 2 \\
\hline $540-600$ & 2 & 1 \\
\hline $600-660$ & 1 & 1 \\
\hline $660-720$ & 0 & 1 \\
\hline$>720$ & 0 & 1 \\
\hline
\end{tabular}

*(Bradley \& Mustard, 2006)

**(Armstrong, 2007) 
Table 5.5. Hydrology Distance Reclassification Schema

\begin{tabular}{|l|l|l|}
\hline $\begin{array}{l}\text { Distance Range } \\
\text { (meters) }\end{array}$ & Percent Influence* & $\begin{array}{l}\text { Alternative Graduated } \\
\text { Schema*** }\end{array}$ \\
\hline $0-60$ & 3 & 9 \\
\hline $60-120$ & 2 & 6 \\
\hline $120-180$ & 1 & 3 \\
\hline$>180$ & 0 & 1 \\
\hline
\end{tabular}

*(Bradley \& Mustard, 2006)

**(Armstrong, 2007)

Table 5.6. Elevation Reclassification Schema

\begin{tabular}{|l|l|l|}
\hline $\begin{array}{l}\text { Elevation Range } \\
\text { (meters) }\end{array}$ & Percent Influence* & $\begin{array}{l}\text { Alternative Graduated } \\
\text { Schema** }\end{array}$ \\
\hline $0-1120$ & 1 & 1 \\
\hline $1120-1310$ & 6 & 5 \\
\hline $1310-1330$ & 10 & 9 \\
\hline $1330-1350$ & 8 & 7 \\
\hline $1350-1390$ & 10 & 9 \\
\hline $1390-1410$ & 7 & 6 \\
\hline $1410-1430$ & 4 & 4 \\
\hline $1430-1550$ & 10 & 9 \\
\hline $1550-1580$ & 5 & 5 \\
\hline $1580-1600$ & 8 & 7 \\
\hline $1600-1630$ & 7 & 6 \\
\hline $1630-1670$ & 2 & 2 \\
\hline $1670-2800$ & 1 & 1 \\
\hline $1710-1760$ & 1 & 1 \\
\hline$>2800$ & 0 & 1 \\
\hline
\end{tabular}

*(Bradley \& Mustard, 2006)

**(Armstrong, 2007) 
Table 5.7. Surface Aspect Reclassification Schema

\begin{tabular}{|l|l|l|l|}
\hline $\begin{array}{l}\text { Aspect } \\
\text { Direction }\end{array}$ & $\begin{array}{l}\text { Aspect Range } \\
\text { (degrees) }\end{array}$ & $\begin{array}{l}\text { Percent } \\
\text { Influence* }\end{array}$ & $\begin{array}{l}\text { Alternative Graduated } \\
\text { Schema ** }\end{array}$ \\
\hline Flat & & 1 & 1 \\
\hline North & $337.5-22.5$ & 1 & 1 \\
\hline Northeast & $22.5-67.5$ & 1 & 1 \\
\hline East & $67.5-112.5$ & 1 & 1 \\
\hline Southeast & $112.5-157.5$ & 1 & 1 \\
\hline South & $157.5-202.5$ & 1 & 1 \\
\hline Southwest & $202.5-247.5$ & 1 & 1 \\
\hline West & $247.5-292.5$ & 6 & 8 \\
\hline Northwest & $292.5-337.5$ & 7 & 9 \\
\hline
\end{tabular}

*(Bradley \& Mustard, 2006)

***(Armstrong, 2007)

All the inputs - the reclassified versions of 1973 Annual Brome Cover, Cultivation, Elevation, Hydrology, Power Lines, Railroads and Roads, and Surface Aspect - are assigned a relative influence and combined into a single dataset. The influence is based on the numbers described by Bradley and Mustard (2006) and adapted by Armstrong (2007); his adaptations were necessary for a total percent influence equal to 100 (see Table 5.8).

Table 5.8. Habitat Suitability Weighted Overlay Parameters

\begin{tabular}{|l|l|l|l|}
\hline Dataset & Parameter * & Influence * & Standardized Influence ** \\
\hline Aspect & NW \& W & $6 \%$ & $6 \%$ \\
\hline $\begin{array}{l}\text { 1973 Annual } \\
\text { Brome Cover }\end{array}$ & 150 meters & $26 \%$ & $28 \%$ \\
\hline Cultivation & 3000 meters & $20 \%$ & $22 \%$ \\
\hline Elevation & $1400-1700$ meters & $10 \%$ & $11 \%$ \\
\hline Hydrology & 60 meters & $3 \%$ & $3 \%$ \\
\hline Power Lines & 1000 meters & $15 \%$ & $16 \%$ \\
\hline $\begin{array}{l}\text { Railroads and } \\
\text { Roads }\end{array}$ & 700 meters & $13 \%$ & $14 \%$ \\
\hline Total & & $93 \%$ & $100 \%$ \\
\hline
\end{tabular}

*(Bradley \& Mustard, 2006)

***(Armstrong, 2007)

The merging of the datasets is initiated using the Weighted Overlay tool. When settingup the tool, two parameters were defined (see Figure 5.8). First, the percent influence was entered in the corresponding \% Influence parameter boxes. Second, the evaluation scale parameter was set to 0 to 100 by 1 ; the final dataset will indicate probabilities of germination ranging from 0 to 100 at an interval of 1 . 

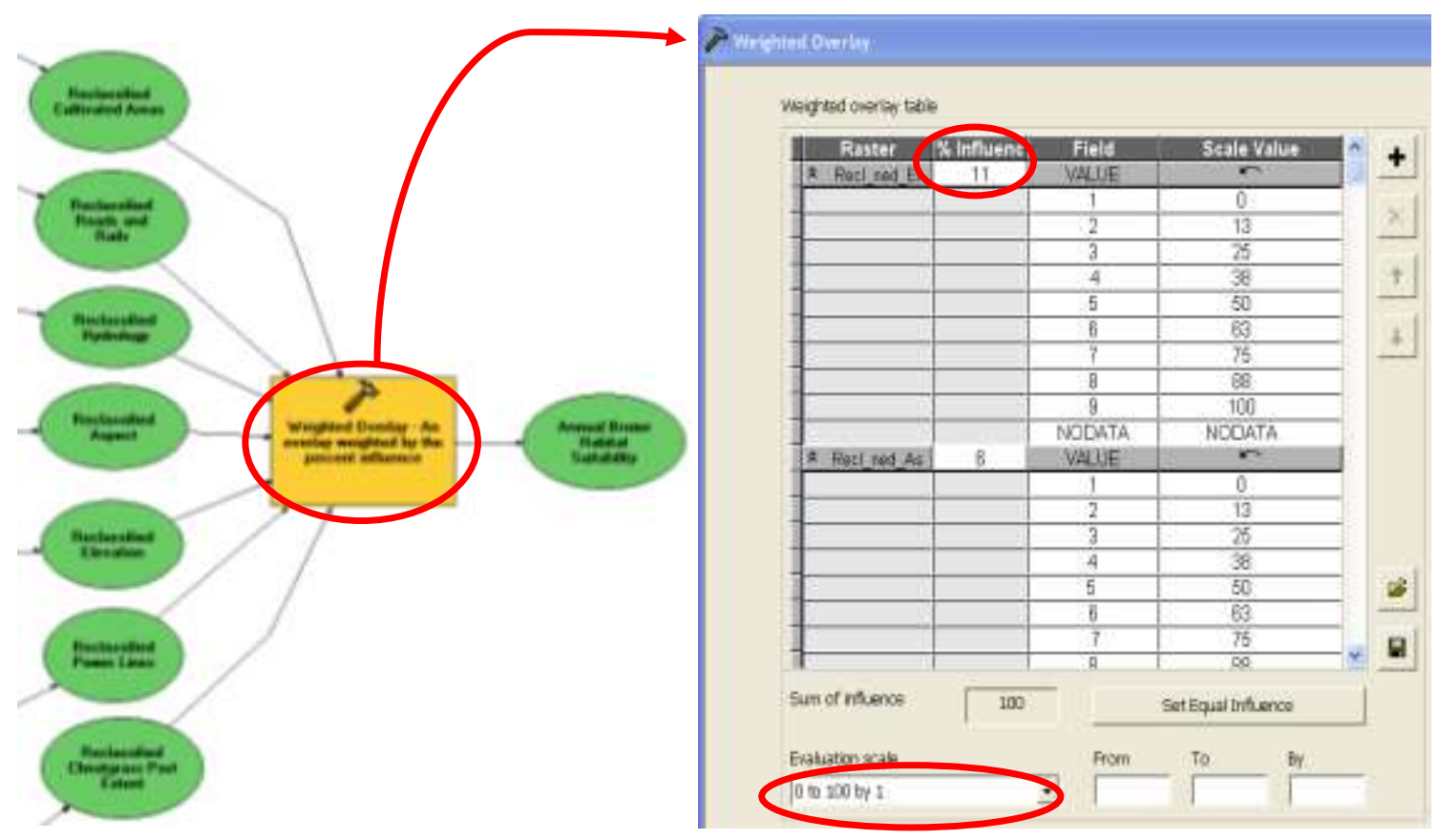

Figure 5.8. Weighted Overlay Tool and its Parameters

\subsubsection{Seed Density Calculation}

To determine the number of agents in a simulation, the seed density throughout the prototype area was calculated (see Figure 5.9). This was accomplished by converting the relative values of the 2001 Annual Brome Cover dataset into plant and seed density values.

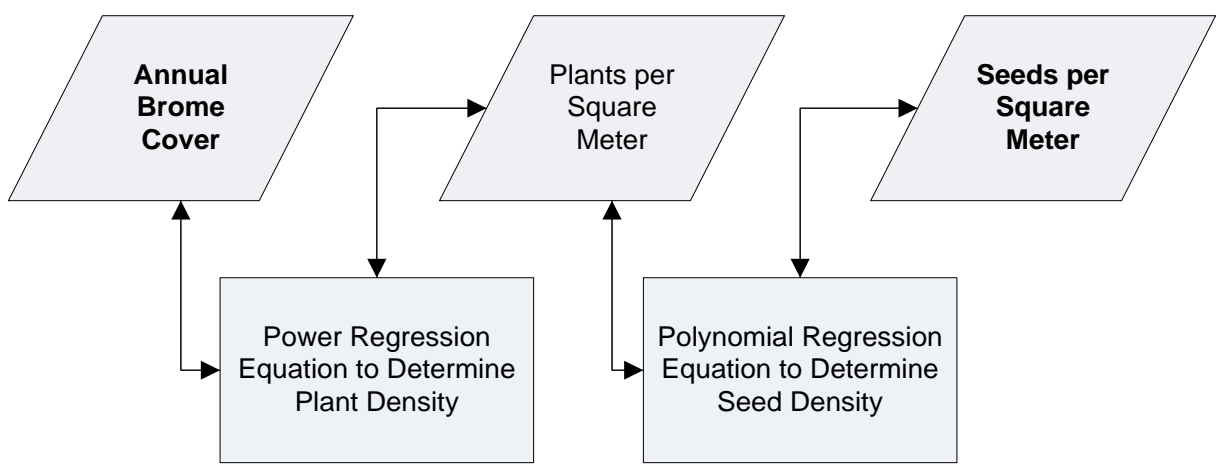

Figure 5.9. Seed Density Calculation Workflow

Since annual brome seed quantities are dependent on the number of parent plants, plant density was calculated first. There are two factors to consider when calculating plant density. First, the 2001 Annual Brome Cover dataset was verified at training sites using ocular estimation; ocular estimation is subjective to the field verifier. Noticeable differences in the percent cover have to increase at a close-to-exponential rate for the user to discern unique values. Second, Hulbert (1955) indicates observed maximum and minimum plant densities. The author concluded, for the purpose of a temporary conversion into a more usable unit, that by applying a power regression equation to those 
minimum and maximum values, the resulting equation would be similar to the subjective nature in which the dataset was verified.

Hulbert (1955) gathered literature pertaining to annual brome up to 1955 . The most significant research took place in Pullman, Washington and Lewiston, Idaho. He indicated the relationship between plant density and seed production (see Figure 5.10). The data included observations of both early and late parent plant germination during the previous year, meaning that more and less developed plants were counted.

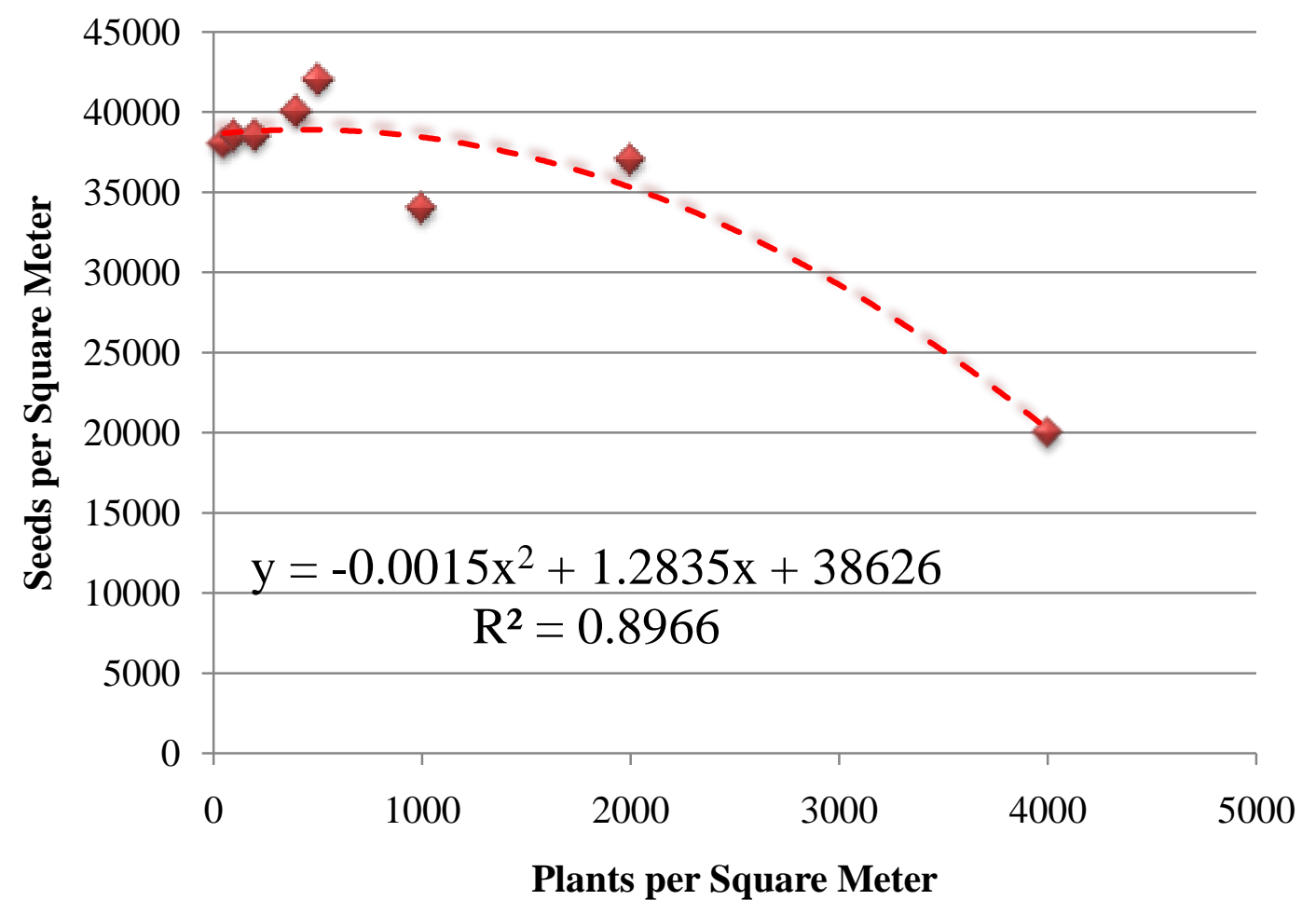

Figure 5.10. The Correlation between Plant and Seed Density

To apply Hulbert's 1955 research, a polynomial regression equation estimates seed density when the plant density is known.

$$
y=-0.0015 x^{2}+1.2835 x+38626
$$

For example, if there was a plant density of 2,000 plants per square meter, then those plants would produce 35,193 seeds per square meter. After the regression equation was established, it was then calibrated to fit the 2001 Annual Brome Cover dataset.

The smallest average plant density measured by Hulbert (1955) was 0.5 plants per square decimeter, whereas the largest was 40 plants per square decimeter. These two values were assigned as minimum and maximum values. The valid percent cover values, 10-40 (see Section 4.3.2 for clarification), were then calibrated in Microsoft Excel with those minimum and maximum values to a power regression equation (see

Figure 5.11); seeds and plants per square decimeter were converted to densities per square meter.

$$
y=0.0345 x^{3.161}
$$




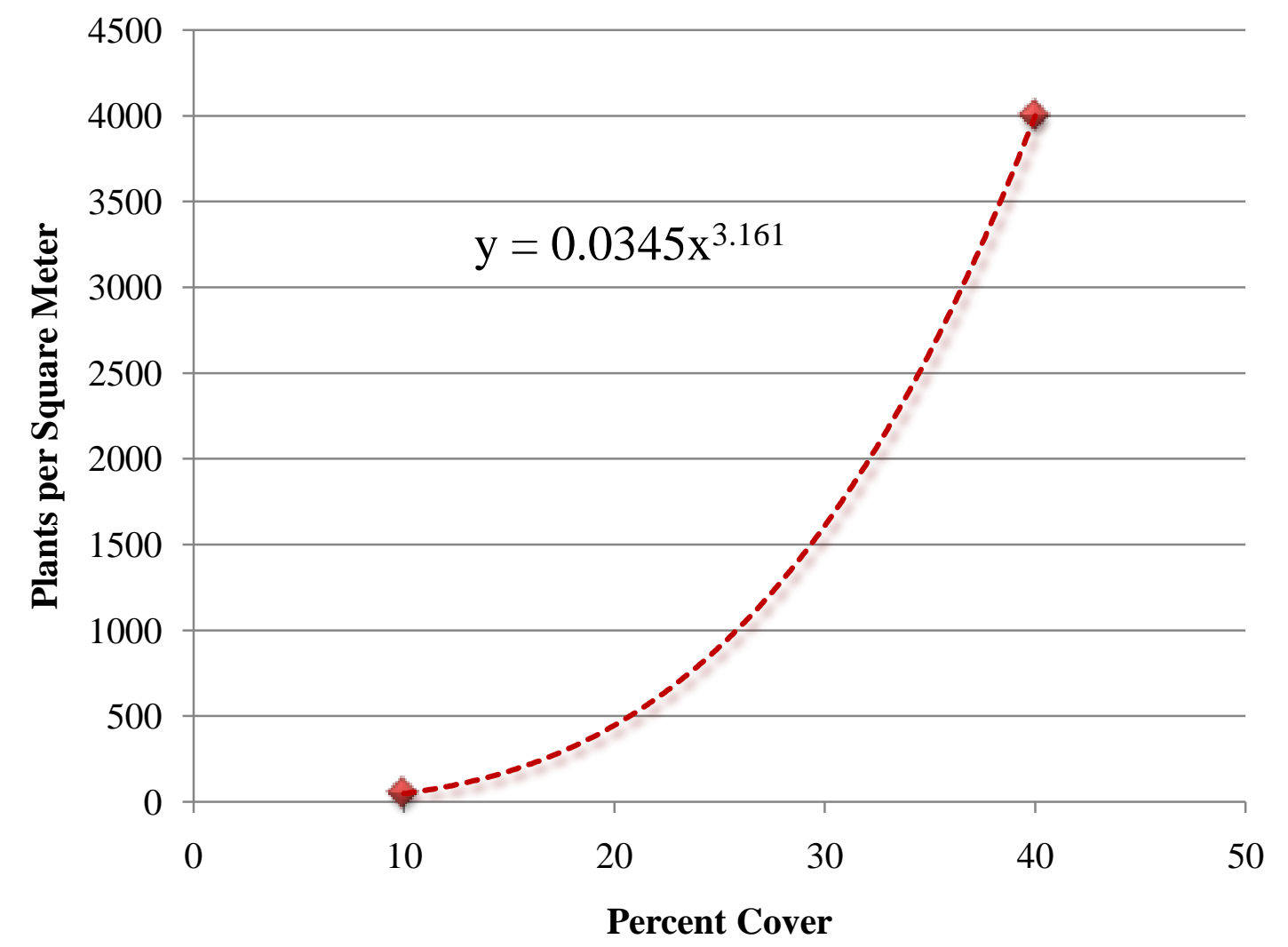

Figure 5.11. The Correlation between Percent Cover and Plant Density

The polynomial and power regressions were combined to form an equation that converts percent cover into a seed value. This, however, neglected percent values below 10 . In the natural environment, there is never a clear cut boundary between two ecotypes but instead fuzzy transitions. Areas with percent cover less than 10, which Peterson (2003) set as a clear-cut boundary between presence and absence, are likely to have some, but relatively unnoticeable, amounts of annual brome. This is why the 10 percent cover minimum value was adapted to fit the fuzzy boundary principle. A power regression equation was applied to a minimum value of 1 and a maximum value of 10 (see Figure 5.12); zero was not used as a minimum because of conflicts it produces in the geoprocessing string (see Chapter 8).

$$
y=100 x^{2.5798}
$$

The maximum value of 10 represented 38,000 seeds per square meter and the minimum value of 1 represented 100 seeds per square meter. 


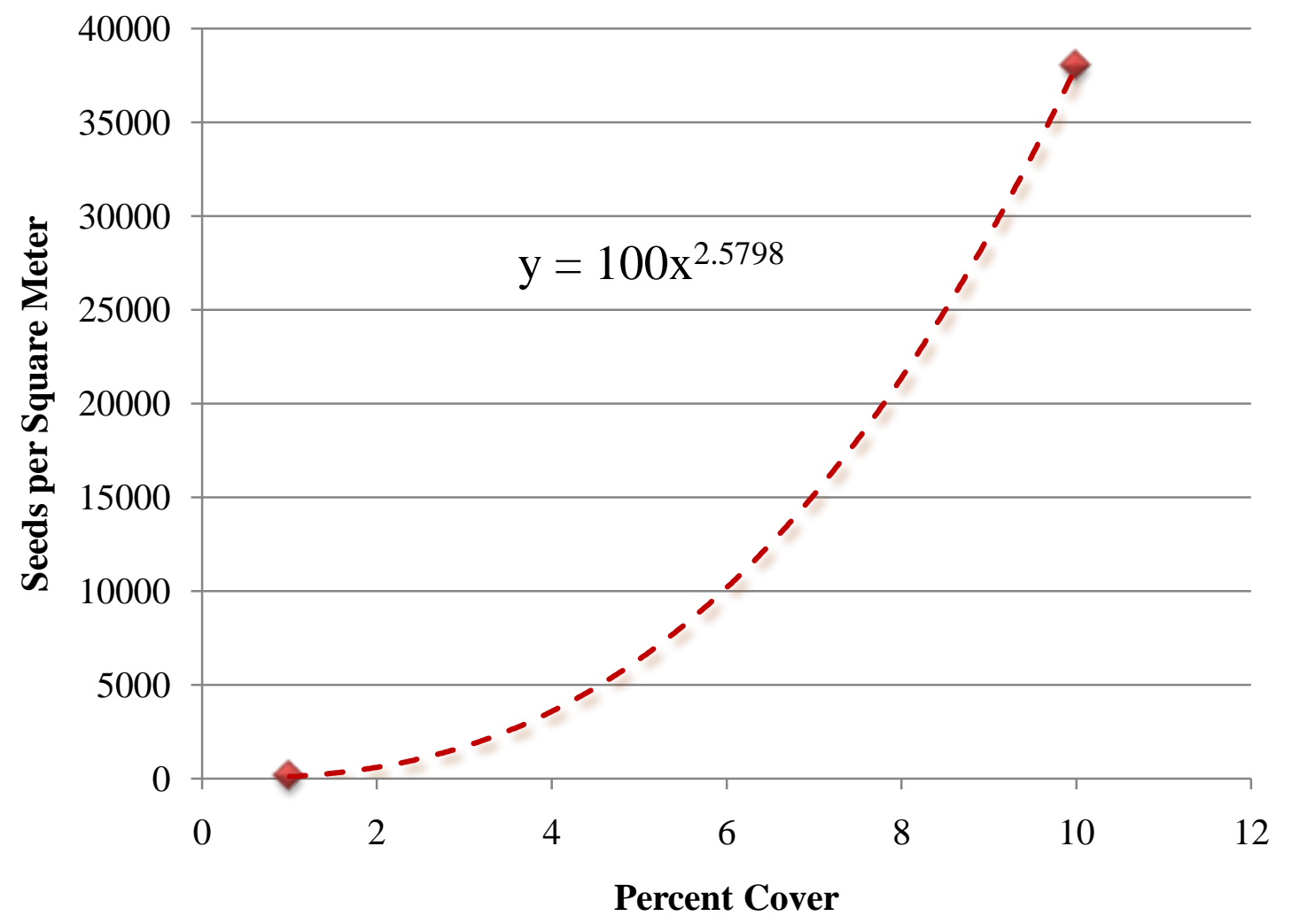

Figure 5.12. The Correlation between Percent Cover and Seed Density for Values Less Than 10

The integration of these equations within ArcGIS was possible using the Single Output Map Algebra tool. This tool takes a single expression built in the Map Algebra language and applies it to a raster dataset. To calculate seed density values for percent cover 1 to 10 and 10 to 40, two separate instances of the tool were necessary. The polynomial and power regression equation combination was set as the Map Algebra Expression parameter for 10 to 40 (Value Set \#1), whereas the stand-alone power regression equation was set as the Map Algebra Expression parameter for 1 to 10 (Value Set \#2; see Figure 5.13). 


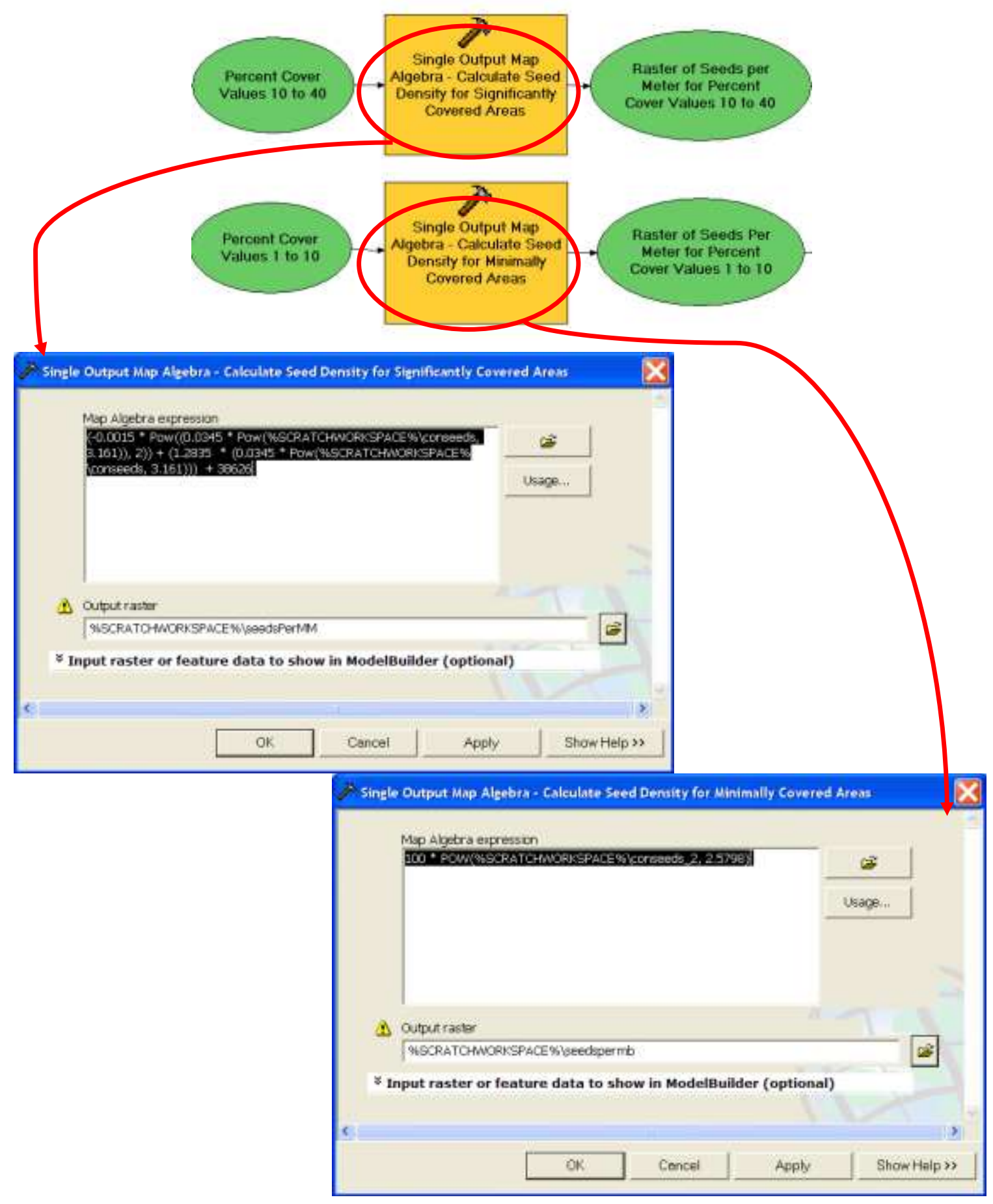

Figure 5.13. Single Output Map Algebra Tools and their Parameters

One group of percent cover values that has so far been ignored contains values equaling zero or greater than 40 (Value Set \#3). To ensure no areas are excluded throughout the entire prototype area, these values need definition. Values of zero indicate no annual brome presence, and so the model was adjusted to give these values a seed density of zero (see Figure 5.14). Values greater than 40 represent anthropogenic structures instead of annual brome cover (see Section 4.3.2); the model was also adjusted to give these 
values a seed density of zero. These adjustments were implemented by adding an empty field to each attribute table and not calculating a corresponding value; by not calculating a value, ArcGIS assumes the value is zero for future calculations.

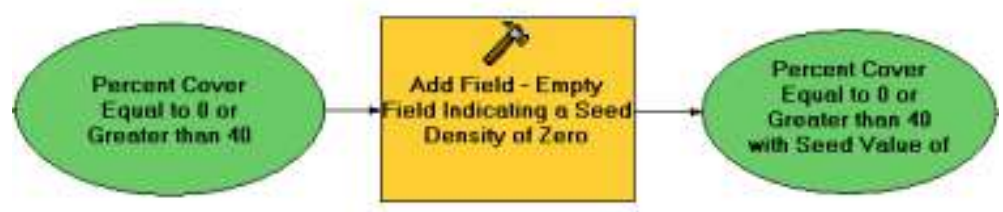

Figure 5.14. Adding an Empty Field

Section 4.3.2 discussed how the 2001 Annual Brome Cover dataset has anomalies degraded raster cell resolution. For the seed density calculations, it is necessary to exclude these outlier areas. Thus a geoprocessing string was created to both exclude the outlier values from the previous three seed density calculations and set the areas to have a seed density of zero.

To set outlier areas to zero, five geoprocessing tools were needed (see Figure 5.15). First, since the user delineates outlier areas, the feature class is conformed to the prototype area extent using the Clip tool. Next, each of the three seed density datasets Value Sets \#1, 2, and 3 - has the areas removed using the Erase tool; only areas outside of the outlier boundary are kept, whereas the areas inside are erased. The last step sets the outlier polygons to have a seed density value of zero by adding an empty field to represent zero. 


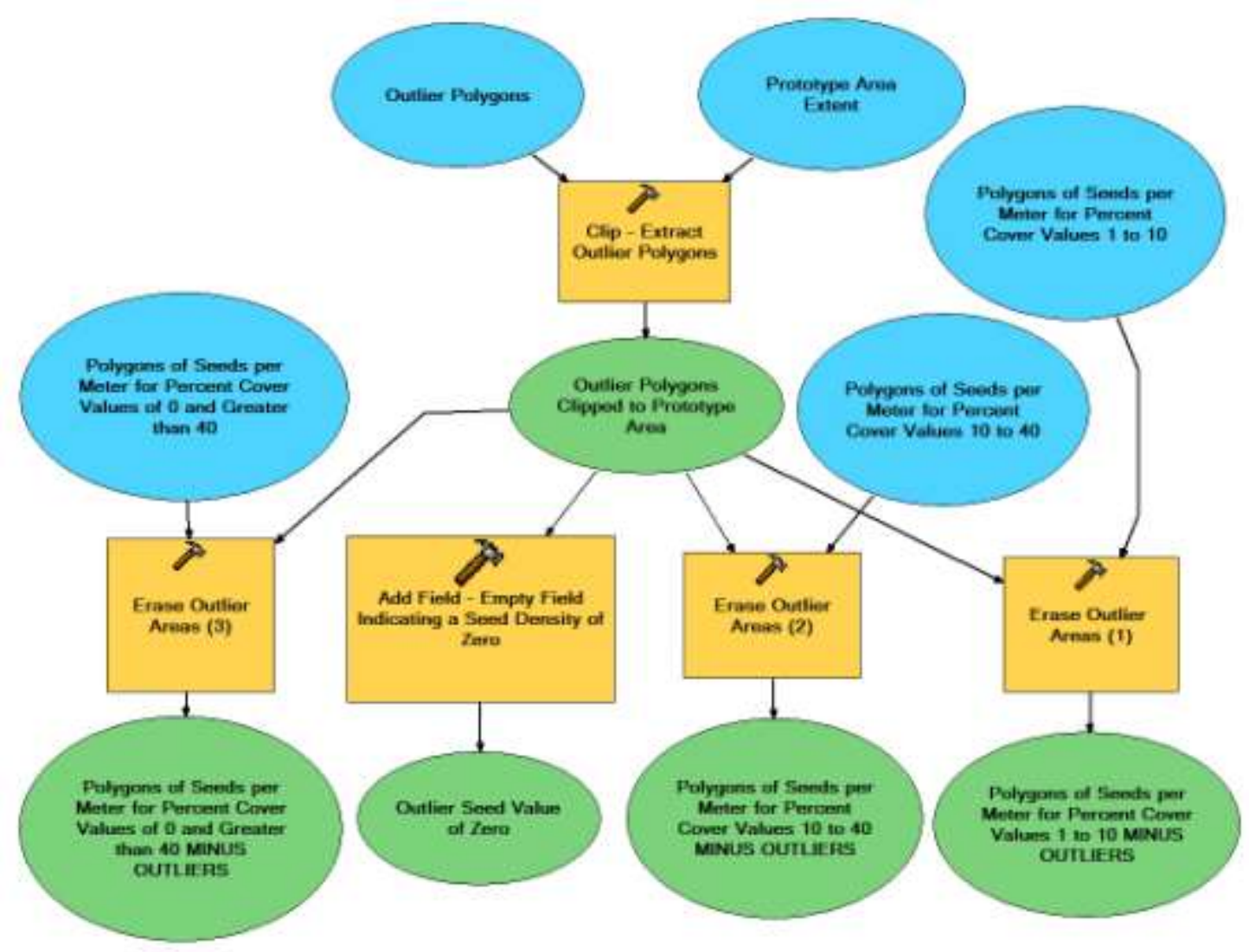

\section{Figure 5.15. Excluding Outliers Geoprocessing String}

The model distinguishes which values to apply a calculation by separating Value Sets \#1, 2, and 3 (see Figure 5.16). This separation is accomplished by conditional statements expressed using the Con tool. When the conditional statement is true for a cell, it is changed to a new value indicated by the parameters. When the statement is false for a cell, it can either be deleted from the dataset or also be changed to a new value indicated by the parameters.

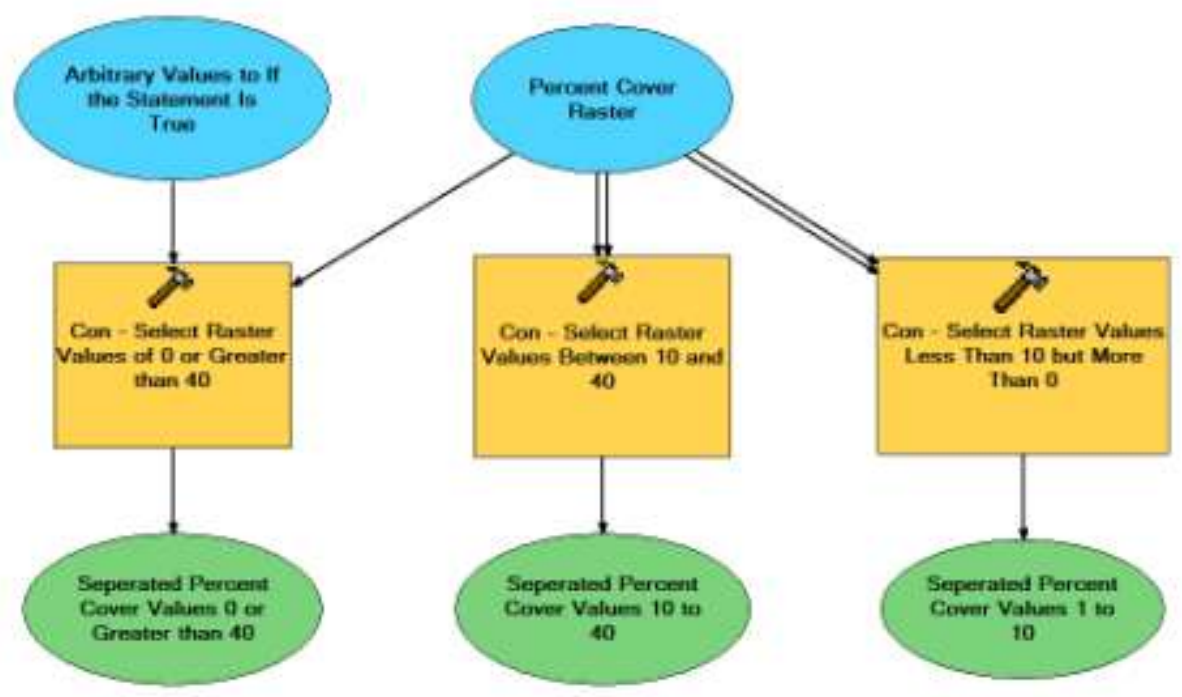




\section{Figure 5.16. Separation Geoprocessing String}

The parameters are set to initiate the separation of the percent cover values (see Figure 5.17). The first conditional statement calls for values equal to zero or greater than 40 . When the statement is true, the cells are set to inherit a value of one, and when the statement is false, the cells are removed. The second conditional statement calls for values greater than or equal to 10 and less than or equal to 40 . The final conditional statement calls for values greater than zero and less than 10 . When the second or final statement is true, the cells are set to inherit the values in the original percent cover raster, and when either statement is false, the cells are removed.

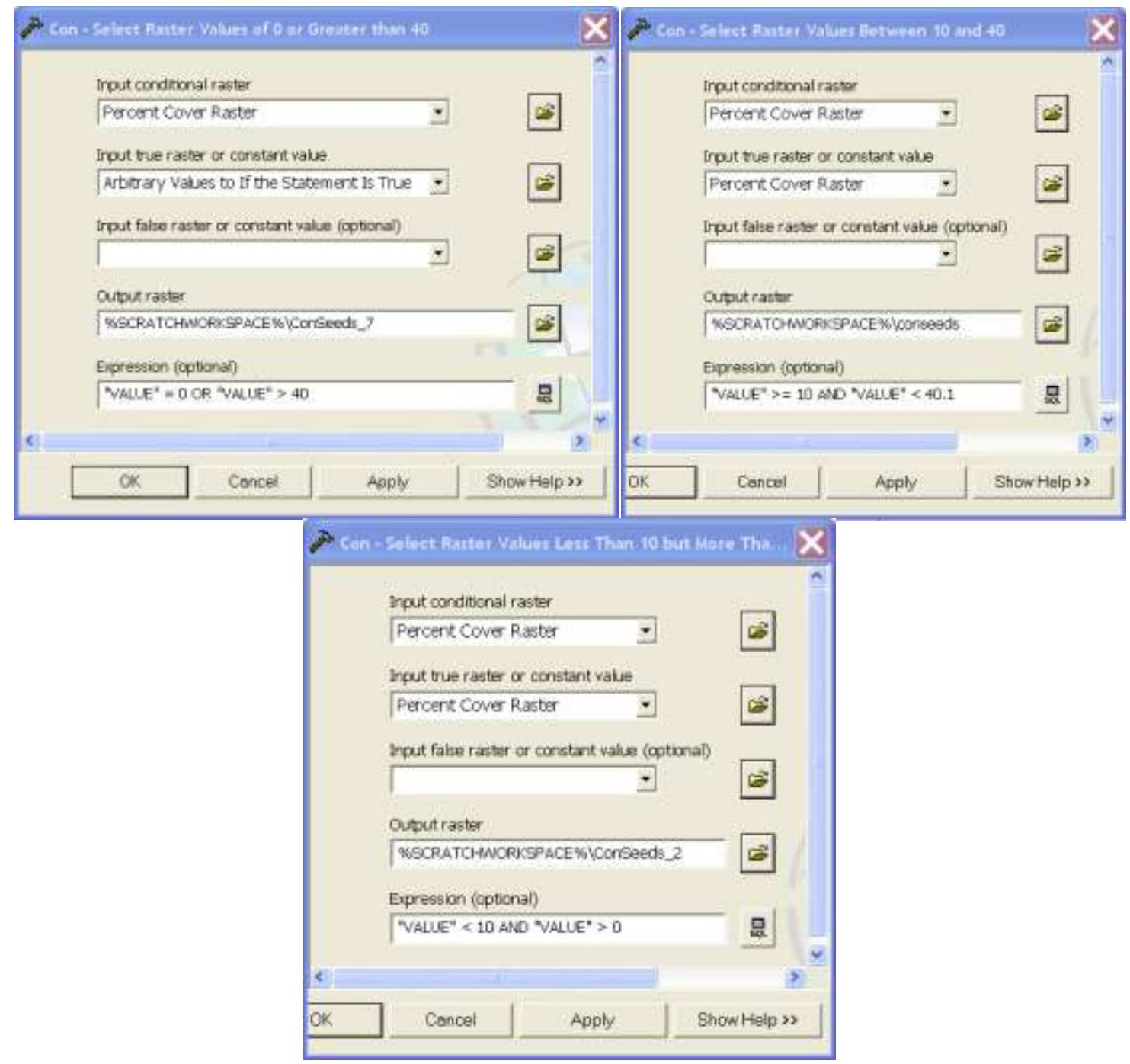

Figure 5.17. Parameters for Con Tools

The separation makes it necessary to recombine the Value Sets after seed densities are calculated. This is why the last part of the model converts the unique rasters into polygon shapefiles and then merges them using the Union tool (see Figure 5.18). To see the entire seed density calculation model, refer to Appendix C. 


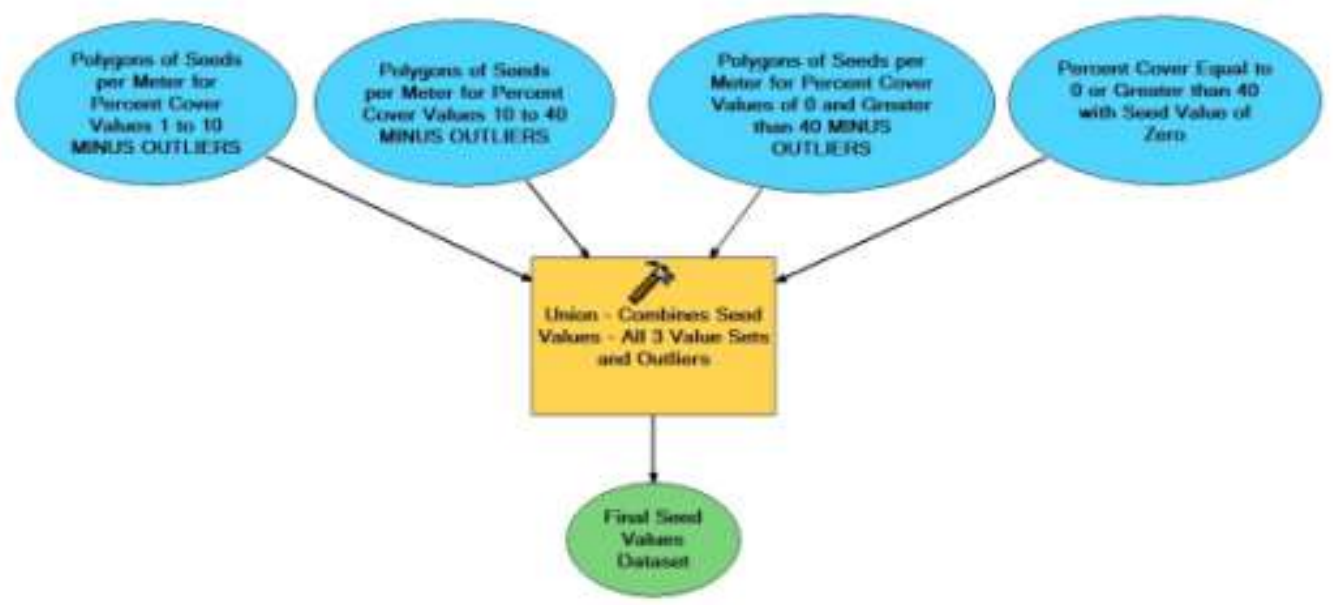

Figure 5.18. Union Tool

\subsubsection{Plant Density Calculation}

The next part of the model has similar components to the Seed Density Calculation, however an exclusive plant density calculation ensures the final dataset inherits a plant density attribute (see Figure 5.19). Unlike the seed density values, the plant density attribute plays an important role in agent movement, as opposed to determining the number agents. The calculated value does not go through a series of inverse conversions at the end the Data Manipulation and Simulation tier of the system architecture, but rather it is used and then deleted from the simulated dataset.

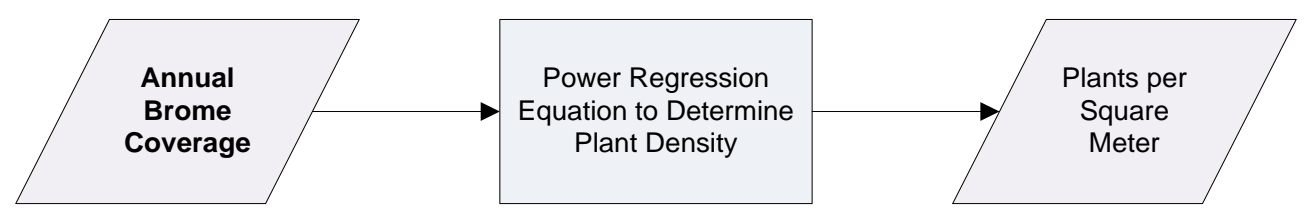

Figure 5.19. Plant Density Calculation Workflow

The power regression equation is used once again to determine the number of plants per square meter.

$$
y=100 x^{2.5798}
$$

As discussed in Section 5.2.3.2, it seemed fitting for this application for two reasons: the ocular estimation verification concept (Peterson, 2003); and Hulbert's (1955) maximum and minimum plant density values. It was concluded that because these plant density values are used as relative barriers of seed movement, and not to calculate further attribute values, their role would improve upon the accuracy of the simulation rather than degrade it.

The integration within ArcGIS was once again possible using the Single Output Map Algebra tool. This calculates the plant density values for a percent cover value between 10 and 40 (Value Set \#4) (see Figure 5.20). 

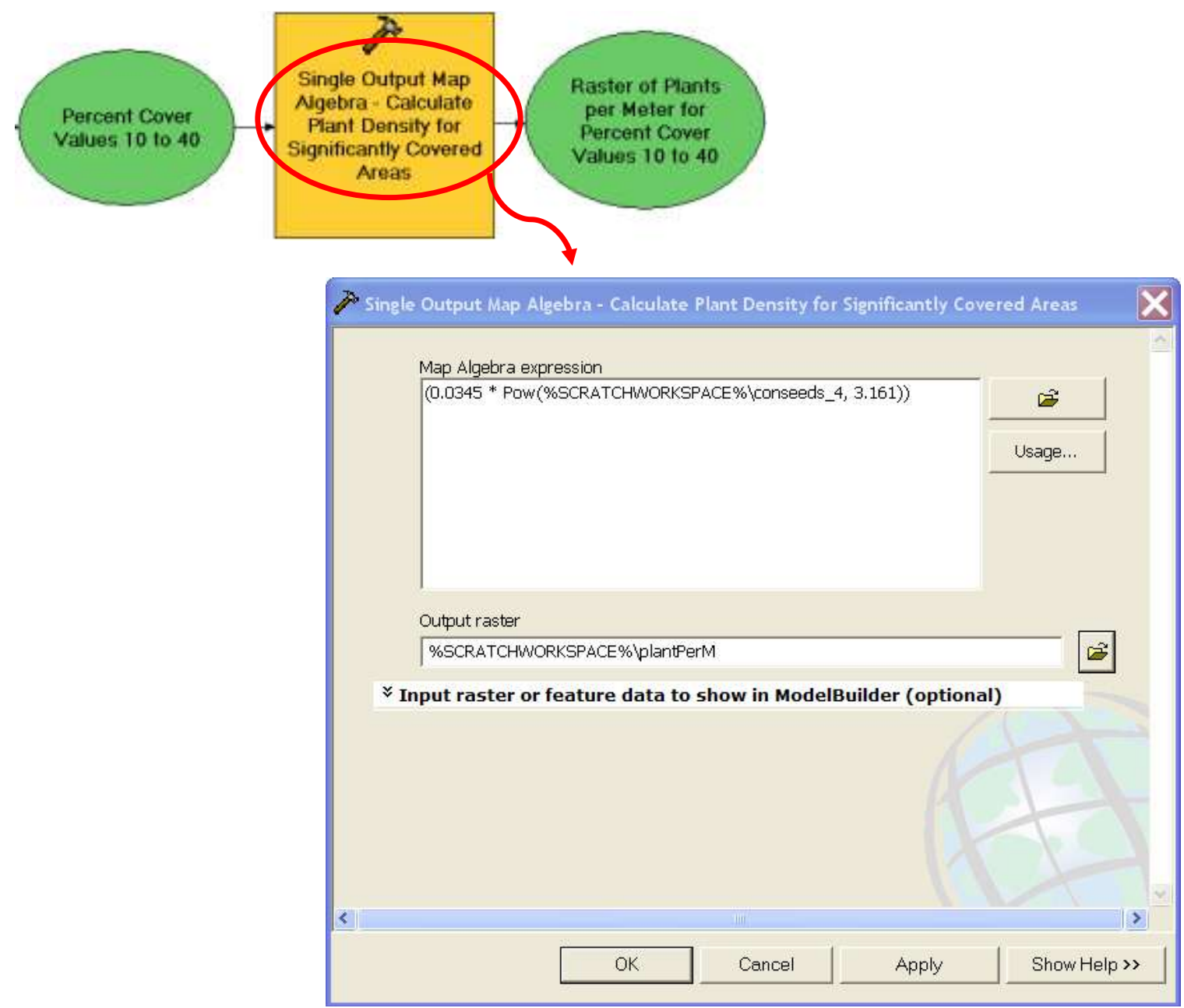

Figure 5.20. Single Output Map Algebra Tool and Its Parameters

Values less than 10 (Value Set \#5) are assigned a density value of one. This signifies areas where there is little to no annual brome vegetation and therefore has no influence on the primary movement. This concept may be confusing because the layman may automatically assume that plots with denser annual brome would have a higher density value. However, this would be incorrect because the value represents seed mobility based on plant density. Denser plots would have less seed mobility and a lower density value. Less dense plots would have more seed mobility and a greater density value. Value Set \#6 is assigned a density value of zero. It represents areas where anthropogenic structures inhibit primary movement.

Like when calculating seed density, to use these different calculations for Value Sets \#4, 5 , and 6 , there needs to be separation. The Con tool achieves this by conditional statements. First, for Value Set \#4, when the percent cover value is between 10 and 40, it is given the corresponding 2001 Annual Brome Cover value. For Value Set \#5, when the percent cover value is less than 10, it is given a value of one, and, for Value Set \#6, when the value was more than 40 , it is given a value of zero. If the conditional statements are not met for any of the three, then the cells are removed from the raster datasets. 


\subsubsection{Customizing Data for Subset Area}

The model has another delineation parameter (see Figure 5.21). This one allows users to select a piece of their study area and test faster than the entire area; this separated portion of the study area is called the subset area or subsection. For example, if a user has a 50 $\mathrm{km}$ by $50 \mathrm{~km}$ study area, but a $5 \mathrm{~km}$ by $5 \mathrm{~km}$ section has a higher importance, he or she simply delineates that area and the model runs quickly. If users do not want to differentiate a subset area, they simply delineate the entire study area.

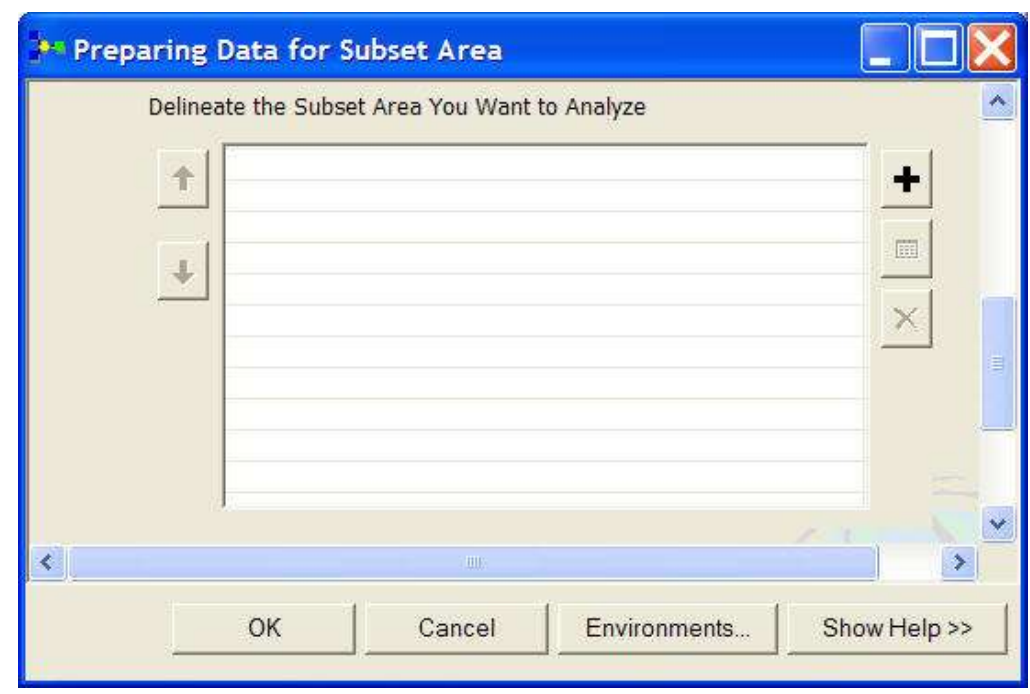

Figure 5.21. Subset Area Parameter Form for Delineation

The new delineation is converted from a feature set to a feature class and then set to clip the layers needed for the remainder of the agent-based model. The Intersect tool then creates the intersection between the data resulting from the habitat suitability model, the seed density calculation, the plant density calculation, and the Wind manipulations. This tool finds the geometric intersection of the four feature classes and creates a new dataset that contains the overlapping attributes. The resulting dataset consists of new polygons that have a unique germination probability, seed density, plant density, and wind value.

\subsection{Preparing Data for Agent Analyst}

The subset dataset needs four more general adjustments before it is ready for the first simulation in AA (see Figure 5.22). For the simulations to work, a division factor is first calculated to customize the number of agents to a user defined amount. Later, with further attribute calculations and by creating random points, agents are placed. After some clean up in the attribute table, deleting extraneous fields, and exporting the shapefile to the Agent Analyst directory, the agents are then ready for primary movement. 


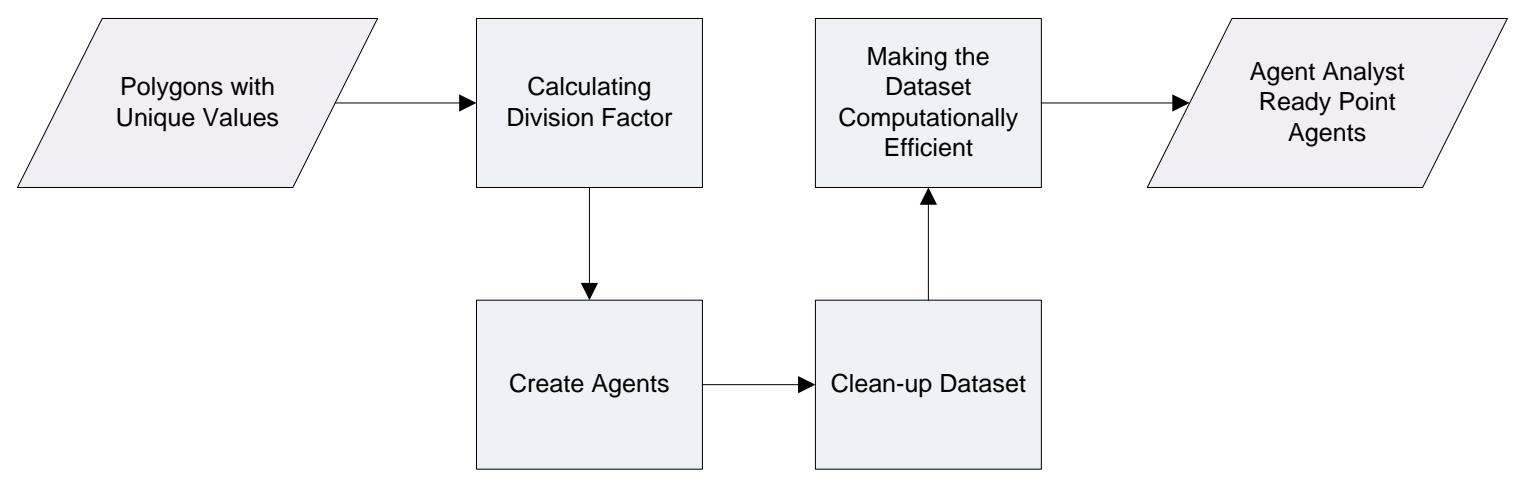

Figure 5.22. Preparing Data for Agent Analyst Workflow

\subsubsection{Calculating Number of Agents}

The number of agents used in a simulation influences the accuracy of the outcome. More agents allow for less seed dense areas to have more representation in the simulation, emulating the natural process more precisely. Quantifying the number of agents in the simulation, however, must be chosen by balancing desired accuracy against available computational capability. As the number of agents is increases, accuracy improves but so does the processing time.

The number of agents used in a single simulation run is dependent on three factors: the seed density values within the study area, the seed density values within the subsection area, and the user defined number of agents. Seed density values are converted to a seed quantity, which will be referred to as seed values. Knowing the seed values for the entire study area is important for uniform accuracy; if the seed values for a subset area were only counted, there would be uneven output accuracies. This is why the methods include parallel calculations of seed values in the study and subset areas.

To calculate the seed values, the polygon areas for both the subset and the study area are needed. This is accomplished by using the Calculate Area script (Giles, 2007) (see Figure 5.23). The script places the area, in square meters, in the attribute field of the corresponding polygon. By finding the product of the area and the seed density, the resulting value represents the number of seeds. 


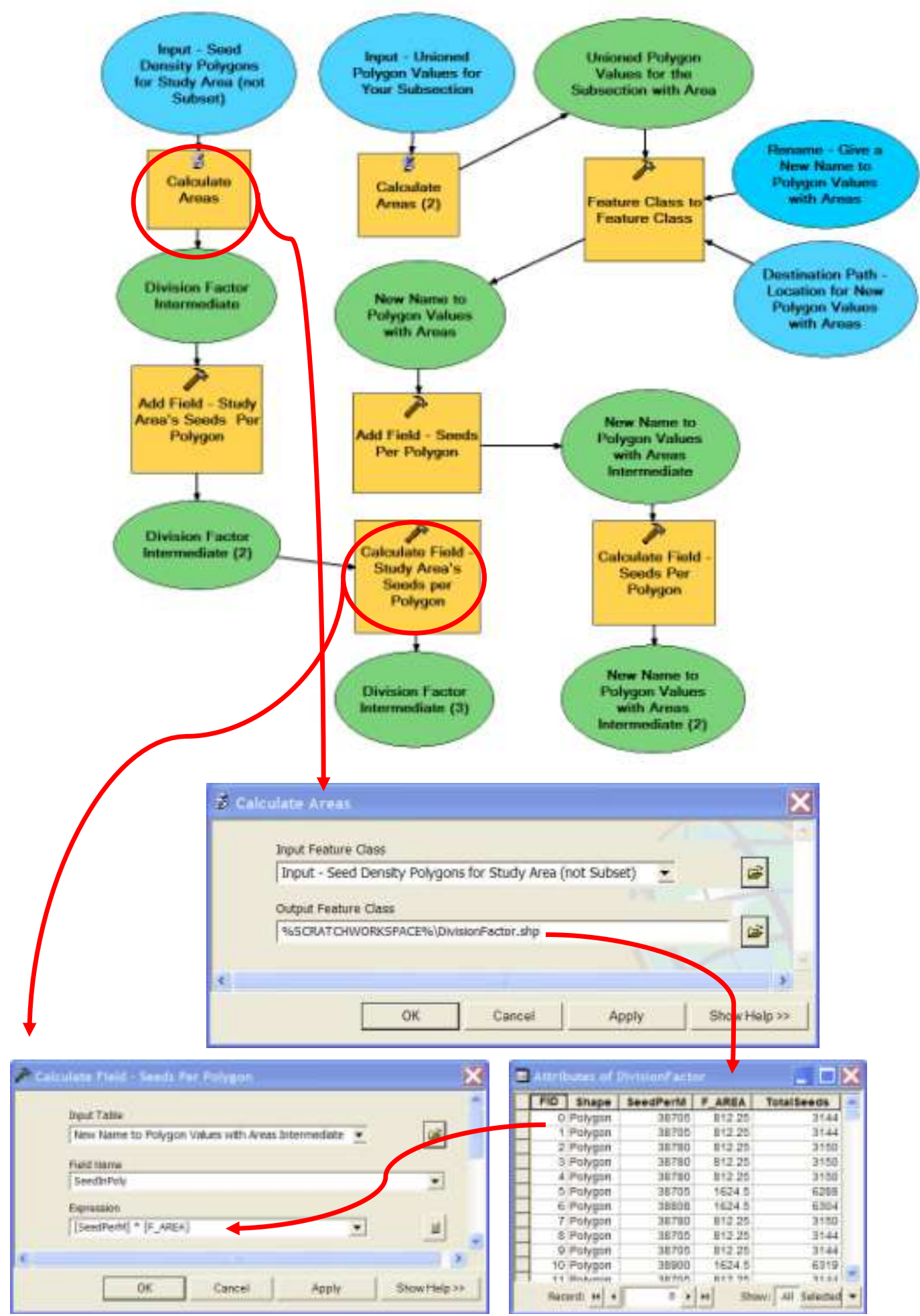

Figure 5.23. The Role of the Calculate Areas Script

Calculating the number of agents is a ratio between the total number of seeds in the study area and what will be called a division factor - a single value that the total seeds will be divided by to result in the user-defined number of agents. For example, if the user delineated a study area with 10 million seeds and wanted only one thousand agents for 
the simulation, the division factor would be calculated by dividing 10 million by one thousand, resulting in a quotient of 10 thousand.

To help with the division factor calculation, a script named Calculate Field Based on a Field's Sum was downloaded from support.esri.com (Giles, 2007); see Appendix G for the script code. The script summarizes the total number of seeds in all the polygons, resulting in a total number of seeds per study area, and then places that sum into a new field, giving each attribute table entry the value. It is this sum that is then divided by the user-defined value, to result in a division factor.

$$
\text { Divison Factor }=\frac{\text { Sum of Seeds in the Study Area }}{\text { The User Defined Number of Agents }}
$$

This division factor is then placed in the subset attribute table and each entry's seed value is divided by it. This resulting value represents the amount of agents per polygon - the sum of all entries/polygons equals the user-defined value. To see the entire Calculate Number of Agents model, see Appendix E.

\subsubsection{Creating Agents}

Once the number of agents per polygon is known, point agents are created within the constraints of each polygon done by the Create Random Points tool (see Figure 5.24). This tool has the parameter option to have a field determine the number of points placed within a parameterized constraint. The number of agents that were calculated earlier in the model is set to determine the number of placed random points. The placement is set to be constrained by the extent of the corresponding polygon.

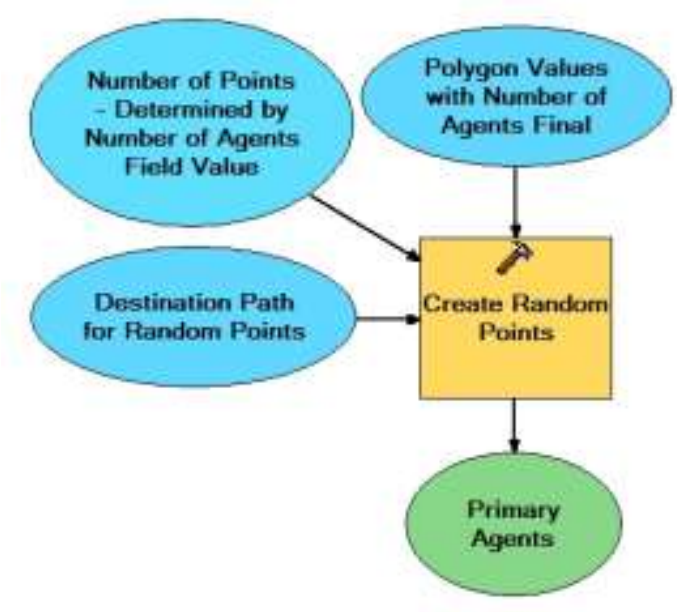

Figure 5.24. Create Random Points Tool

The random points inherit none of the attributes of their constraining polygons. To accommodate this void, a join operation between the points and polygon values allows for attribute inheritance. Each point inherits all of the calculated attributes of the polygon it was constrained by. Of particular importance are the wind and plant density values, which will be used in the primary movement simulation. 


\subsubsection{Clean Up Dataset/Make Computationally More Efficient}

Although some instances of the Calculate Field tool are important for calculating attribute values that will be used in the simulations there are some, in combination with other field tools, that merely clean the attribute table. Certain geoprocesses often do not allow for custom field naming, requiring this clarification step. Throughout the model there are multiple Add Field, Calculate Field, and Delete Field geoprocesses, which are meant to serve the purpose of assigning logical field names and deleting extraneous, often confusing ones.

\subsubsection{Multiple Trials}

After testing of the complete model, which will be discussed in further detail in Chapter 8 , it was determined that an average of multiple runs creates a more reliable final dataset. After calculating the number of agents, the model is split into ten trials. Each trial created the number of agents at different random locations within the same polygon constraint. It is not until the post-processing steps of the Data Manipulation and Simulation system architecture tier that the trials are recombined and averaged. It should be assumed that until the post-processing, each geoprocess is carried out per trial.

\subsection{Primary Movement Simulation}

The last geoprocess of the Preparing Data for Agent Analyst model involve exporting the point agents into Agent Analyst's program directory. The dataset is linked to the agentbased model. The Primary Movement Simulation can then use the dataset's attributes in the code. After the code has been run, the primary agents' positions are directly edited (see Figure 5.25).

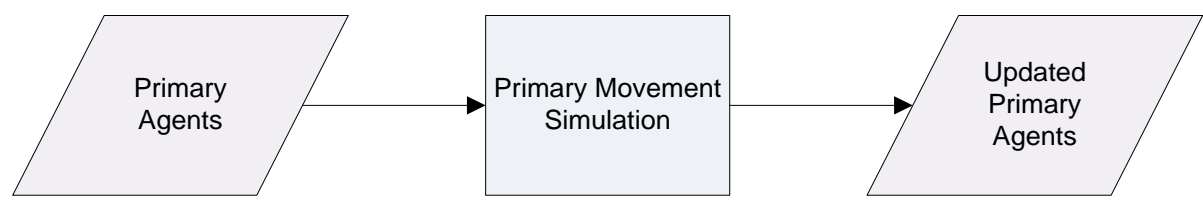

Figure 5.25. Primary Movement Simulation Workflow

\subsubsection{Configuring Agent Analyst}

As described in Section 3.5.4, before agent actions can be implemented the agents must be linked to a dataset (see Figure 5.26). This is done by setting the vector agent data source to the dataset's path name. The vector agents then inherit the attributes stored in the attribute table of the shapefile. The geometry attribute is manipulated in the code to change point positions. 

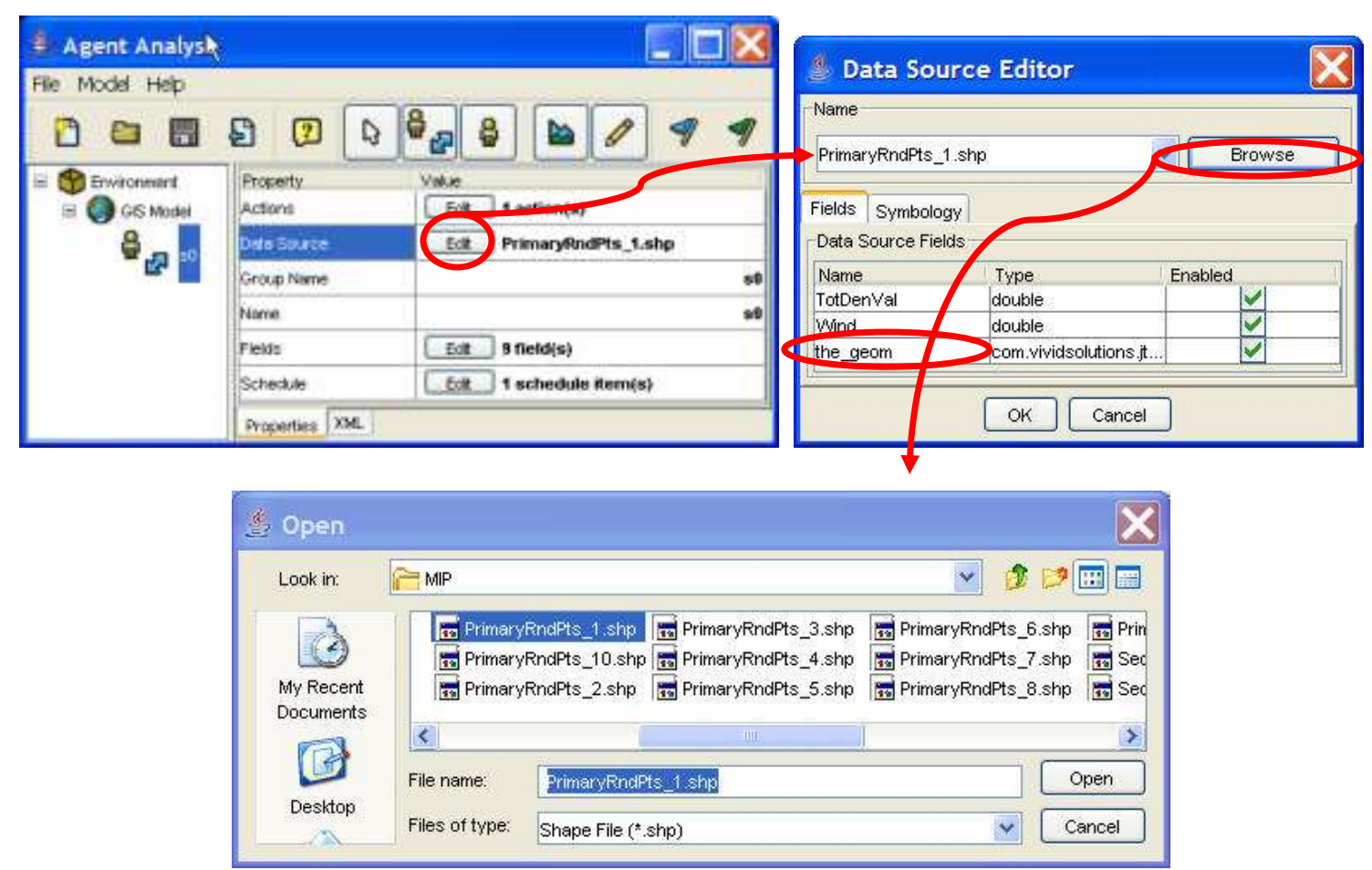

Figure 5.26. Vector Agent's Data Source Editor

The model also needs to know which dataset it will be editing. The writeAgents model action was modified to allow the vector agents to edit the linked shapefile (see Figure 5.27). In the case of primary movement, the agents name is $\mathrm{s} 0$ and the pathname is ./projects/MIP/PrimaryRndPts_1.shp; as stated before, the shapefile needs to be in the project folder within the AA directory and this is why the pathname starts with a period.

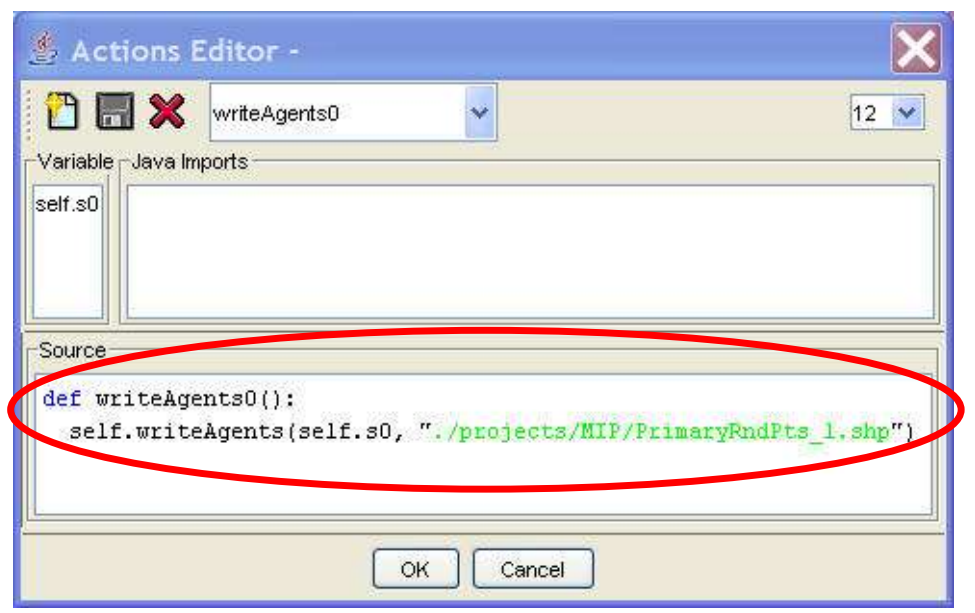

Figure 5.27. Model's Actions Editor

\subsubsection{Primary Movement Simulation: Code for Agent Actions}

The primary movement code is based on two principles: the wind and plant density attribute, and randomness. Environmental processes are often thought to exhibit random behavior when viewed at a macroscale. For example, precipitation quantities may determine if a plant lives or dies but it is also important to consider the overall rainfall 
pattern, how much water that plant needs to survive, how much water the soil has retained, arrangement of the plants surrounding it which may provide shade in the hottest parts of the day and reduced the amount of transpiration, etc. When all of these important deterministic factors are combined to complete a complex formula of the natural processes, it would seem somewhat random.

Randomness is used in the written code, and determines whether an agent moves and, if so, in which direction. Random variables are generated within AA by creating a numerical variable for the double precision field in the Field Editor and assigning it to a randomly generated number; the random number generator method is Random.uniform.nextDouble().

The code starts by determining if randomness allows a seed to move (see Figure 5.28). To imitate this, a random number is compared to the wind and plant density values. In the case of wind, strong winds in a particular direction have a value of one, whereas weaker winds have a value of zero. A random number is generated and if it is less than the wind value, the wind was not strong enough to carry the agent. However, if this random number is more than the wind value, the wind was strong enough to carry the agent.

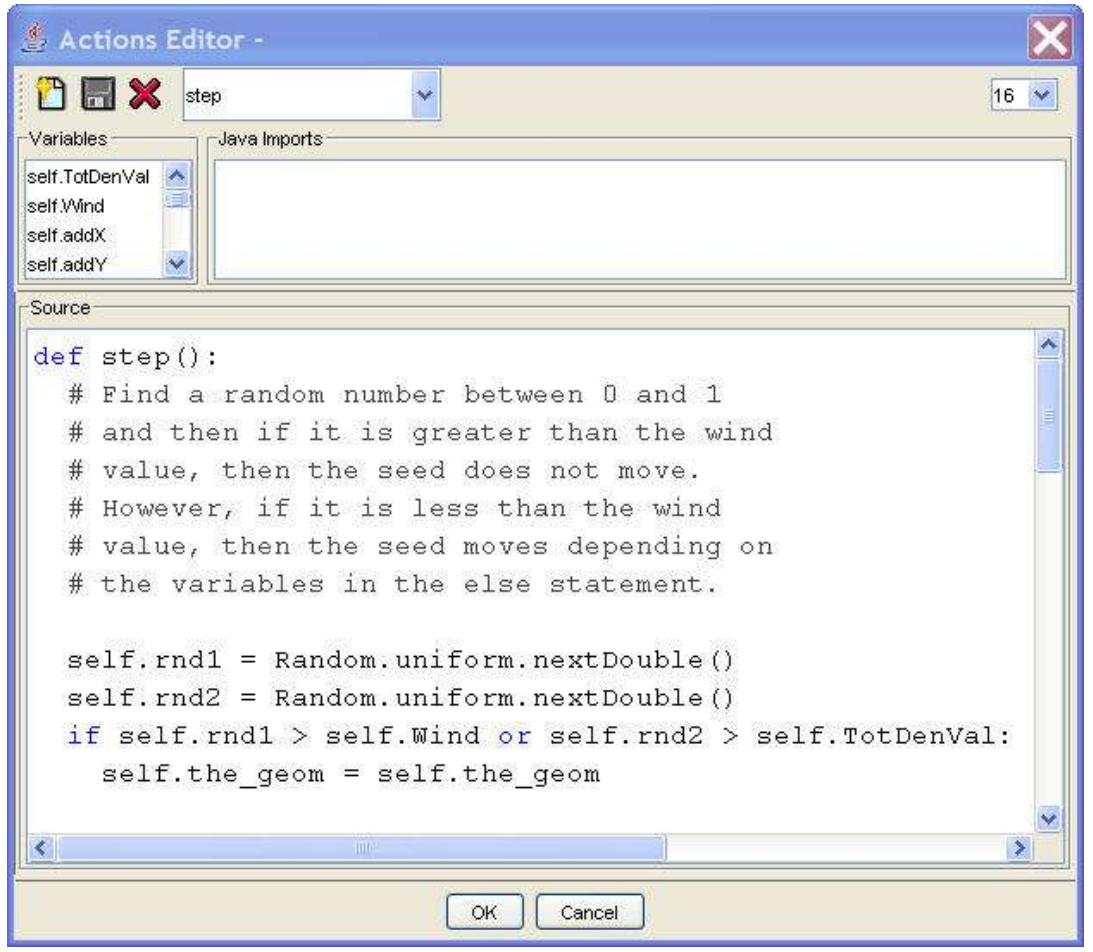

Figure 5.28. Vector Agent's Actions Editor

In the case of plant density, it must be determined if the inhibition force is greater or less than randomness; the total plant density value calculated in Section 5.2.3.3. If the plant density is less than a randomly generated number, then that plot is too dense for seed movement. If the plant density is more than a randomly generated number, then the density was too insignificant to affect movement. 
The travel distance of each seed agent correlates with the research collected by Hulbert (1955). He explains that seeds primarily travel on the ground surface and are not carried into the air like pollen. Because annual brome seeds are rough and heavy, it makes long distance dispersal by natural forces difficult. Hulbert's research indicated that seed movement seemed to be fairly localized and only a few seeds reach more distant locations (see Table 5.9).

Table 5.9. Distance and the Seeds Collected

\begin{tabular}{|c|c|c|}
\hline Distance (m) & Seeds Collected & Standard Value \\
\hline 1 & 15 & 0.75 \\
\hline 3 & 0 & 0 \\
\hline 5 & 0 & 0 \\
\hline 10 & 4 & 0.20 \\
\hline 15 & 0 & 0 \\
\hline 25 & 1 & 0.05 \\
\hline
\end{tabular}

There is a trend of less seeds at increasing distances. Outliers in the data, however, interrupted a direct correlation by having a single seed collected 25 meters and four seeds collected 10 meters from the parent plants. And since the sample size is so small -20 seeds - one seed has a five percent influence.

\section{A logarithmic regression indicated a negative correlation between seed presence and distance away} from parent plant (see

Figure 5.29). As the distance from the parent plant increases, the number of seeds decreases. Furthermore, the regression equation suggested a maximum travel distance of approximately 15 meters for a single seed and a minimum of one meter. Since the minimum is one meter and not zero, the wind value and plant density values are initially compared to a randomly generated number to indicate if there is any travel; if there is travel, then the regression equation is applied to the agent. 


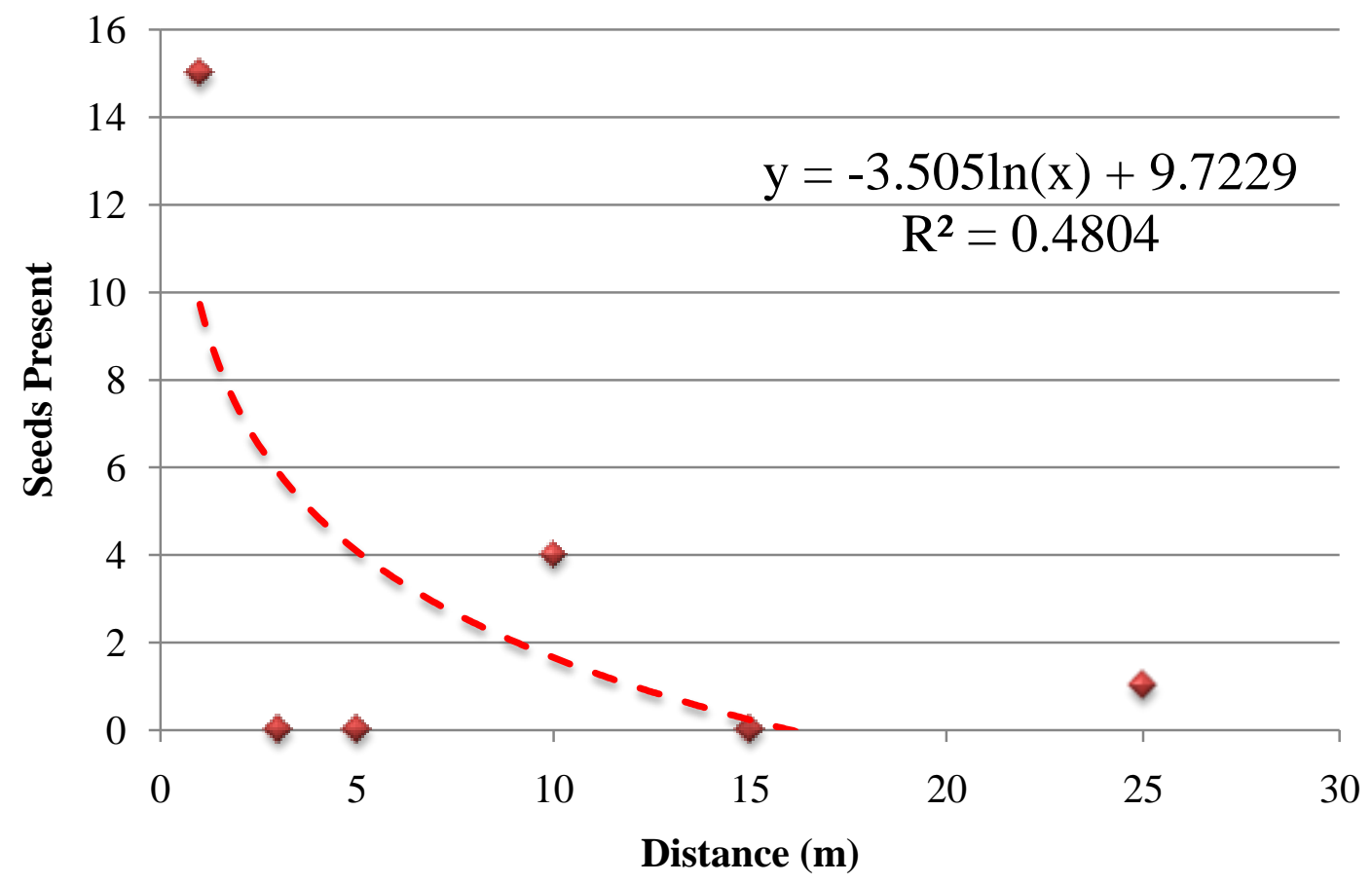

Figure 5.29. Correlation between Distance and Seeds Collected

Unfortunately, little research has been done investigating dispersal as far as the number of seeds reaching certain distances. The accuracy of applying a logarithmic regression also raises a caution flag. In ecology, depending on the phenomena being studied, a correlation coefficient greater than 50 to 60 percent is typically acceptable. The dataset published in Hulbert (1955), however, does not meet that requirement nor does it have a large enough sample size to explain a low correlation coefficient -0.4804 . Future work updating this equation with more valid data will be discussed in Chapter 11.

To apply the regression equation in AA, the standard values were plotted (see Figure 5.30). This resulted in a seed percentage reaching a distance rather than absolute values. The logarithmic regression was essentially the same for the structure and correlation coefficient, but the y-axis values changed. 


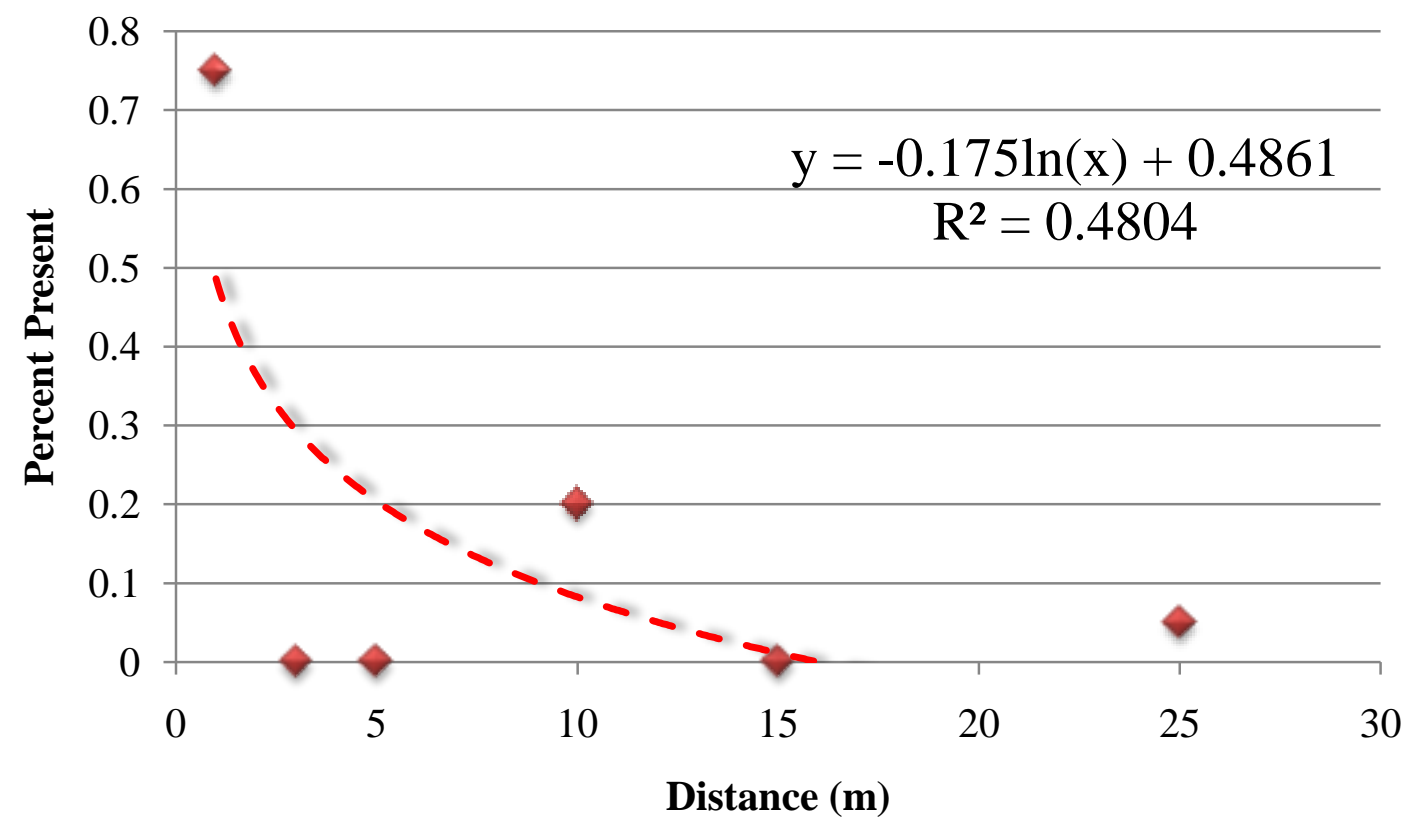

Figure 5.30. Correlation between Distance and Standard Value

Once adapted, the equation was in the format to implement in the agent code (see Appendix I). In the code, the inverse of the regression equation was broken down into two sections; the inverse was necessary to solve for $\mathrm{x}$.

$$
\text { Seed Distance }=\frac{1}{e^{(\text {Wind Value } \times \text { Plant Density-0.4861)/-0.175 }}}
$$

The first section calculates the product of the wind and plant density value and substitutes $y$ (see Figure 5.31). The second finds the exponent of y after conducting the remainder mathematical functions. 


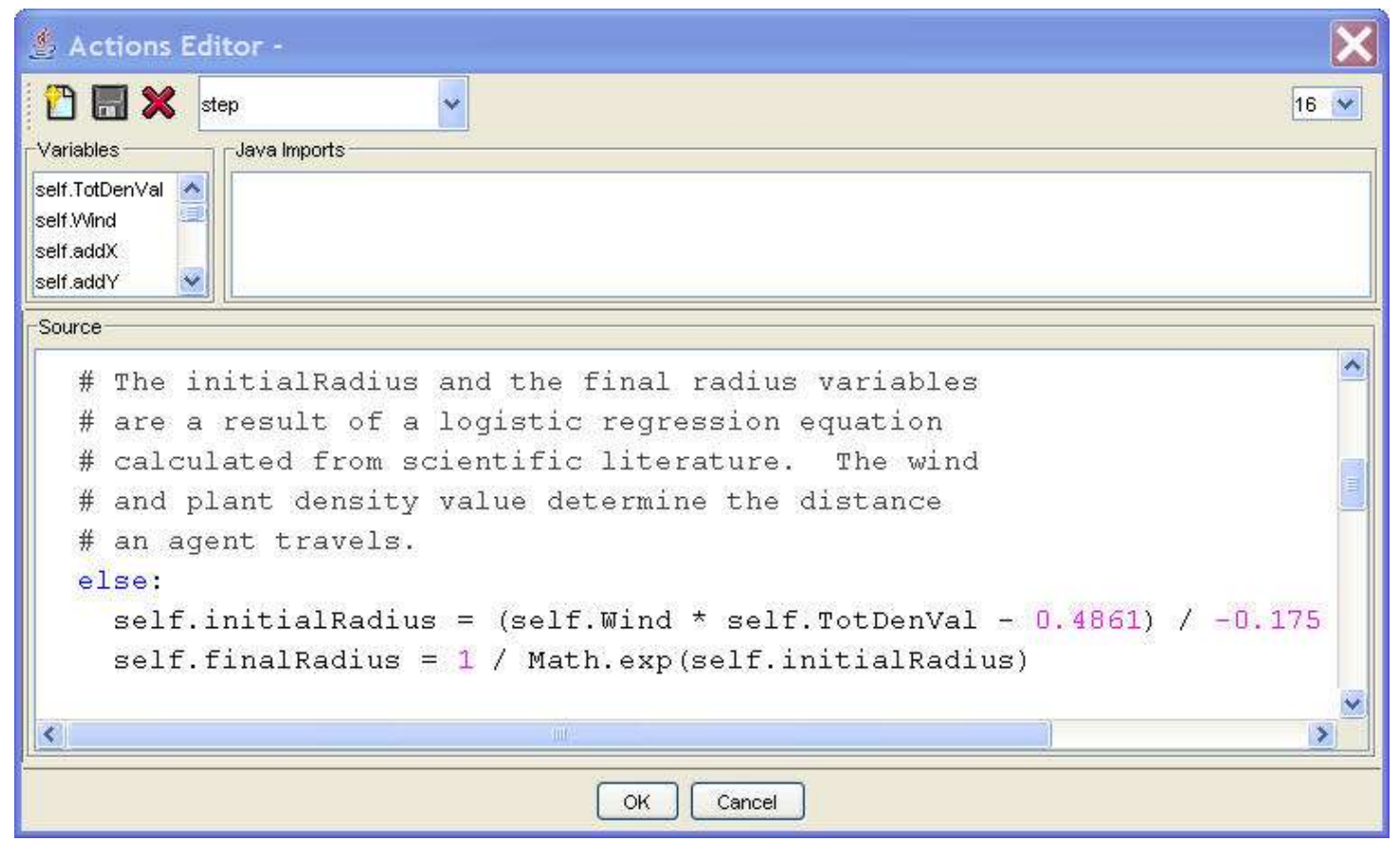

Figure 5.31. Calculating Seed Distance Implemented in the Action Editor

To implement both of these parts, two double precision numeric variables were created using the Field Editor. The first was named initialRadius. This variable is assigned the value of the equation's quotient before finding the inverse exponent. The second was named finalRadius. This variable is assigned the exponent of the quotient; the final distance the seed will travel. The term radius signifies that the direction has not yet been determined.

Direction is perceived in two different ways: the direction in which one agent travels and the flow of all agents. The direction in which an individual agent travels is a function of randomness (see Figure 5.32), whereas the flow is dependent on the attribute barriers. An agent has a random chance of moving at a specific bearing, which is no more likely than another bearing. To program this, two more double precision numeric fields were created using the Field Editor. The first was assigned a random value. The second, sprdDirD, was assigned the product between the random value and 359.9. This product produces a random direction in degrees. 


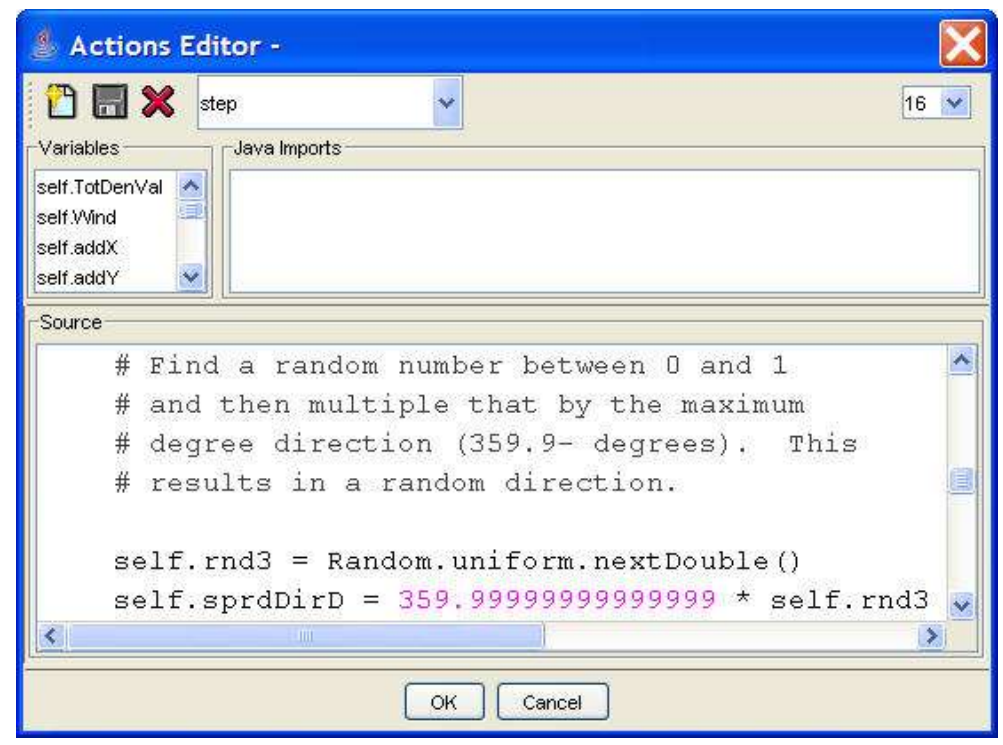

Figure 5.32. Calculating Seed Travel Direction Implemented in the Action Editor

To edit the position of an agent, the direction and distance are used to calculate a change in Euclidean space. The direction is converted into radians, a format more compatible with trigonometry functions.

$$
\text { Direction in Radians }=\frac{\text { Direction in Degrees } \times \pi}{180}
$$

The direction in radians and the final travel distance can then be used to calculate changes in $\mathrm{X}$ and $\mathrm{Y}$ coordinates (see Appendix $\mathrm{J}$ for a conceptual graphic). The change in the $\mathrm{X}$-coordinate is calculated first. This value is computed using the cosine function. The cosine of theta is simply the adjacent length over the hypotenuse length

$$
\cos (\theta)=\frac{\text { Adjacent }}{\text { Hypotenuse }}
$$

By substituting the variables that are known, the cosine of the direction in radians equals the change in $\mathrm{X}$ over the travel distance.

$$
\cos (\text { Direction in Radians })=\frac{\Delta x}{\text { Travel Distance }}
$$

By then algebraically rearranging the equation variables, the change in $\mathrm{X}$ can be solved by multiplying the travel distance by the cosine of the direction in radians.

$$
\Delta x=\cos (\text { Direction in Radians }) \times \text { Travel Distance }
$$

With a value assigned to the change in $\mathrm{X}$, the more complicated change in the $\mathrm{Y}$ coordinate can be calculated and is defined using the Pythagorean Theorem.

$$
a^{2}+b^{2}=c^{2}
$$

By substituting the hypotenuse with the travel distance and one of the sides with the change in $\mathrm{X}$, the remaining side can be calculated.

$$
\Delta x^{2}+\Delta y^{2}=(\text { Travel Distance })^{2}
$$


Then by algebraically rearranging the variables, the change in $\mathrm{Y}$ can be solved by taking the square root of the travel direction squared minus the change in $\mathrm{X}$ squared.

$$
\Delta y=\sqrt{(\text { Travel Distance })^{2}-\Delta x^{2}}
$$

Calculating the change in $\mathrm{Y}$ has one more step because of the square root and power functions used inadvertently convert negative changes in $\mathrm{Y}$ to positives. The change in $\mathrm{Y}$ calculation was separated into two cases. First, when the direction (degrees) is more than zero and less than 180 - values in quadrants I and II of the Euclidean plot - the change in $\mathrm{Y}$ is positive and the equation is left alone. Second, when the direction is not more than zero or not less than 180 - values in quadrants III and IV of the Euclidean plot - the change in $\mathrm{Y}$ value is negated.

$$
\Delta y=-\sqrt{(\text { Travel Distance })^{2}-\Delta x^{2}}
$$

Programming changes in $\mathrm{X}$ and $\mathrm{Y}$ involved organizing new variables into one if-then statement. Three double precision numeric variables were dimensioned in the Field Editor: sprdDirA, addX, and addY. The sprdDirA is set to the degrees-to-azimuth conversion's quotient; addX inherits the change in $\mathrm{X}$ and add $\mathrm{Y}$ inherits the change in $\mathrm{Y}$ when the program is compiled and run. The if-then statement, written to distinguish positives and negatives, encapsulates these variables and equations that determine their values (see Figure 5.33).

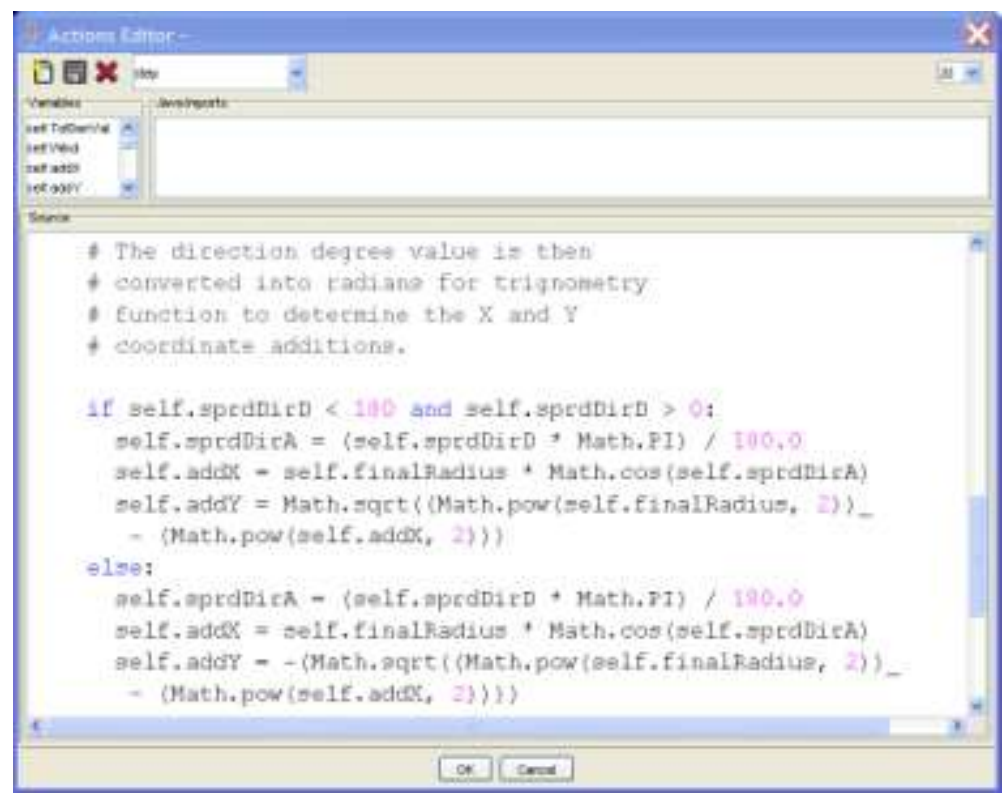

Figure 5.33. Calculating Change in $X$ and $Y$ Implemented in Actions Editor

After the changes in $\mathrm{X}$ and $\mathrm{Y}$ are calculated, the actual agents' positions are edited. This is done by adding the change in $\mathrm{X}$ and $\mathrm{Y}$ to the original $\mathrm{X}$ and $\mathrm{Y}$ coordinates. For example, if the agent is headed five meters due north, the change in $\mathrm{Y}$ will be five. If the agent is headed five meters due south, the change in $\mathrm{Y}$ will be negative five. This is then added to the $\mathrm{Y}$ coordinate, northing, and $\mathrm{X}$ coordinate, easting, changing the agent's position. 
The change is programmed by inserting the geometry field into the code and no variable needs be established using the Field Editor. By simply setting the $\mathrm{X}$ coordinate geometry equal to it plus the change, the new $X$ coordinate is derived (see Figure 5.34). The same process works for the $\mathrm{Y}$ coordinate.

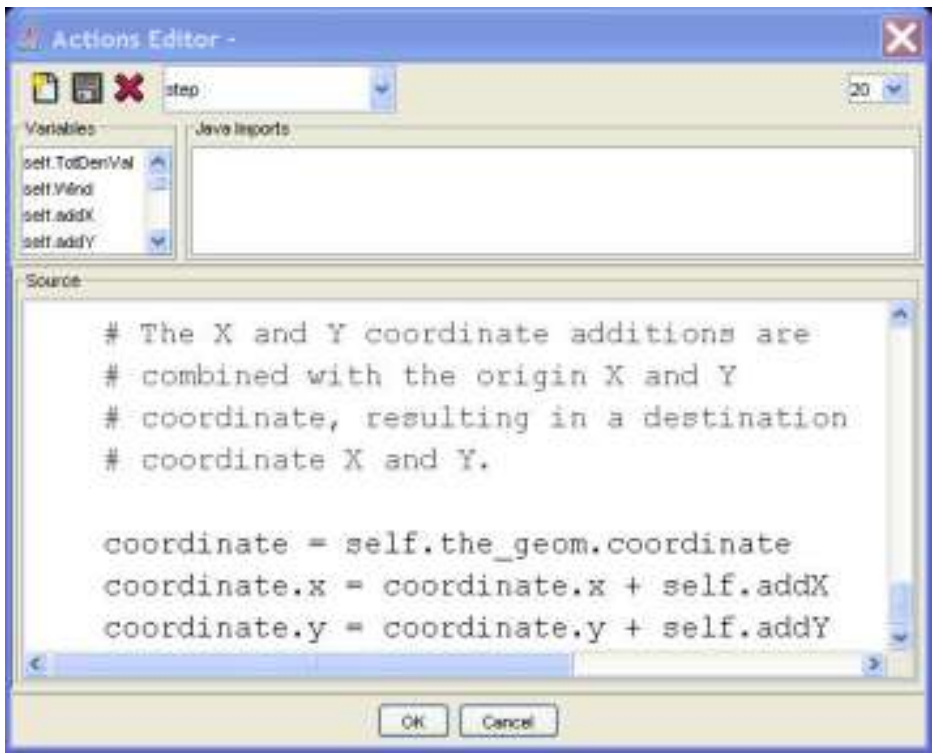

Figure 5.34. Calculating Final $X$ and $Y$ Coordinate in Actions Editor

\subsection{Secondary Movement Simulation}

The Secondary Movement Simulation takes place in ArcGIS Desktop. By importing the shapefiles updated by the Primary Movement Simulation from the Agent Analyst directory, they can be further manipulated by secondary movement. The portion of the road dataset that was clipped in the Customizing Data to the User Defined Extent part of the model will determine if an agent will travel further.

The simulation has four basic steps (see

Figure 5.35):

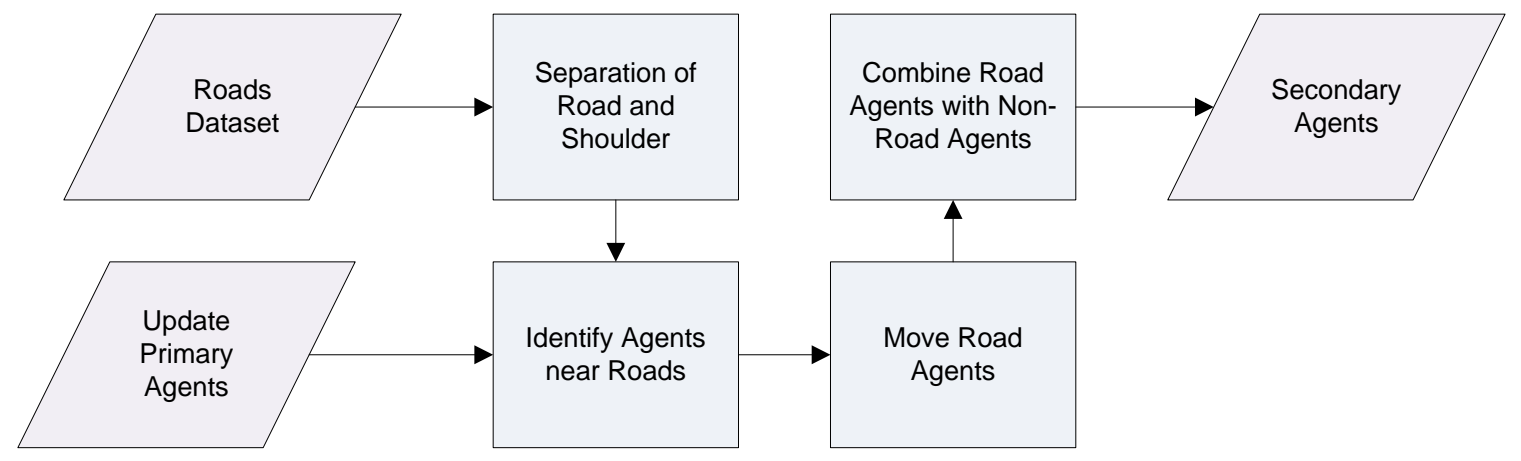

Figure 5.35. Secondary Movement Simulation Work Flow

1) The road dataset is prepared by distinguishing a road's shoulder from the actual road and by giving the roads width by converting them into polygons.

2) The agents that are within the boundary of the road and its shoulder are identified. 
3) The identified agents continue through a series of geoprocesses that will give them a new location based on random placement.

4) The newly placed agents and the unmoved, non-road, agents are recombined into a single dataset and exported back to the AA directory for the Germination Simulation.

\subsubsection{Separation of Road and Shoulder}

After the seed's primary movement, it has reached its final location only if it is not within a meter of a road feature. If a seed is within a meter, considered within the shoulder, secondary movement occurs. To simulate this seed movement, both the road and the shoulder must be considered. Separating these two is a necessary step.

First, two Buffers tools create two unique buffers: one that is the width of a single lane highway, 2.1336 meters, and one that extends one meter from the edges of this road (see Appendix K). Buffer overhang is avoided by clipping them to the extent of the subset area. The first initial buffer - the width of the road plus one meter - then follows two paths. The first identifies agents that are within its constraints (buffer \#1). The second erases a portion of its interior using the erase tool and the normal-sized road buffer as the erase feature. The remainders are two one meter sections (buffer \#2) (see Figure 5.36).

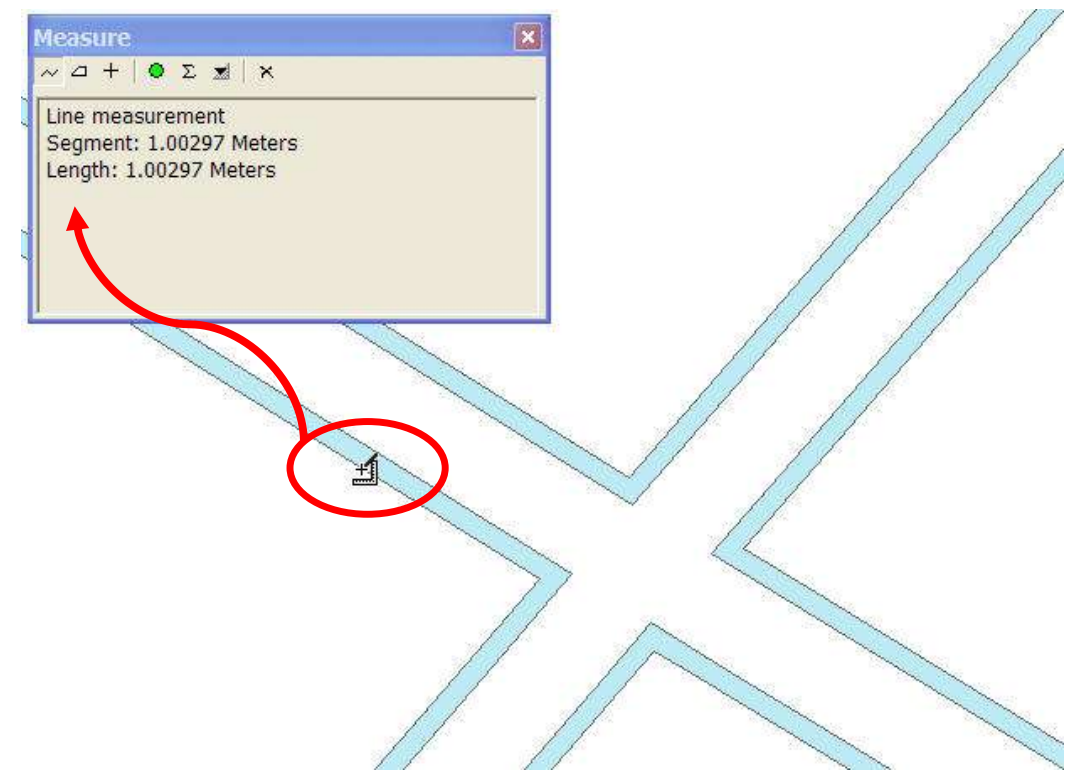

Figure 5.36. Width of Buffer \#2

\subsubsection{Identify Agents Near Roads}

When creating the buffers, agents within the extent of buffer \#1 are identified. But before the buffer is linked to the agents, a field is added to its attribute table. That field is assigned a value of one, which will be used to identify agents. Once the buffer's attribute table is configured, the Identity tool has the agents within buffer \#1 inherit that buffer value (see Figure 5.37); each agent that is within the buffer has a value of one. The sum of all the buffer values represents the total number of agents within buffer \#1 and will 
exhibit secondary movement. The Summary Statistics tool is used to generate this summary value.

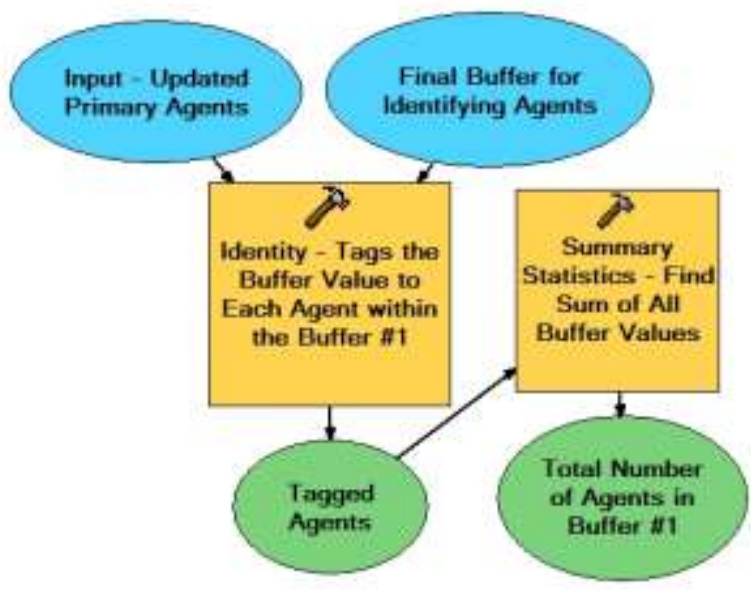

Figure 5.37. Identity and Summary Statistics Tools

\subsubsection{Move Road Agents}

The sum of the total number of agents within buffer \#1 represents the number of seeds that "move". Random points are created within the constraints of buffer \# 2 by using the Create Random Points tool (see Figure 5.38). The amount of random points is dependent on the number of agents summarized after being identified. For example, if 100 agents were identified within buffer \#1, then 100 new random points are generated within the constraint of buffer \#2.

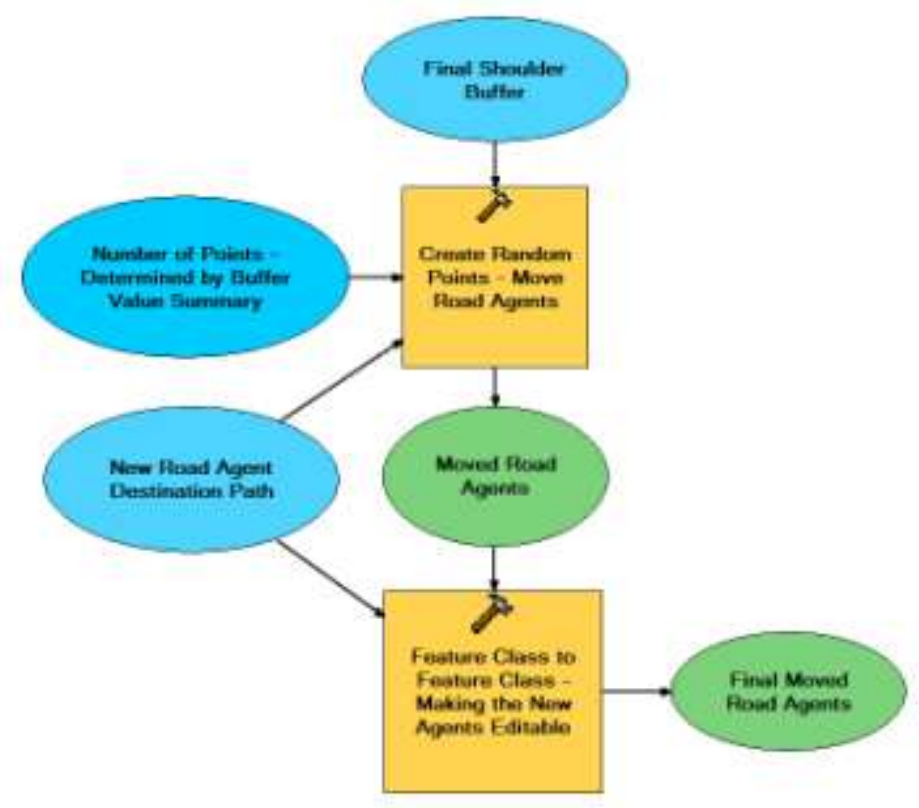

Figure 5.38. Create Random Points Tool 


\subsubsection{Combine Road Agents With Non-Road Agents}

After new road agents have been placed within the constraints of buffer \#2, the dataset is recombined with the non-moved agents. This involves two steps. The first erases all the agents within the buffer \#1 extent through using the Erase tool, which results in areas without agents. These areas then gain new random agents by recombining that agent dataset and the "moved" agent dataset using the Union tool.

\subsection{Seed Germination Simulation}

The last geoprocess of the Secondary Movement Simulation model involve exporting the point agents into Agent Analyst's program directory. The dataset is linked to the agentbased model and then the Seed Germination Simulation can use the dataset's attributes in the code. After the code has been run, the secondary agents' positions are directly edited (see Figure 5.39).

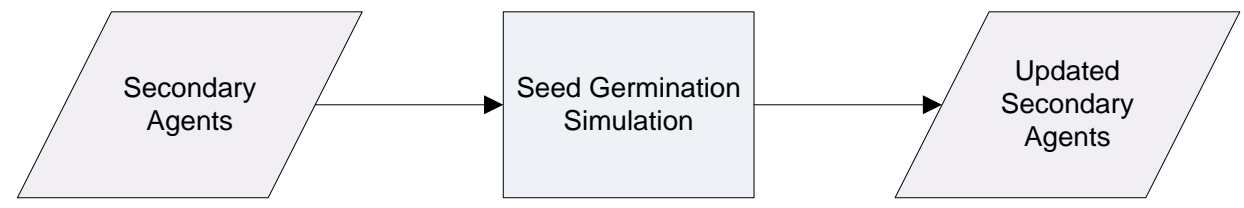

Figure 5.39. Seed Germination Simulation Workflow

\subsubsection{Configuring Agent Analyst}

As described in Section 3.5.4 and 5.4.1, the agents must be linked to the dataset before agent actions can be implemented. This is done by setting the agent's data source to the secondary agent path name (see Figure 5.40). The vector agents then inherit the secondary agent attributes. 


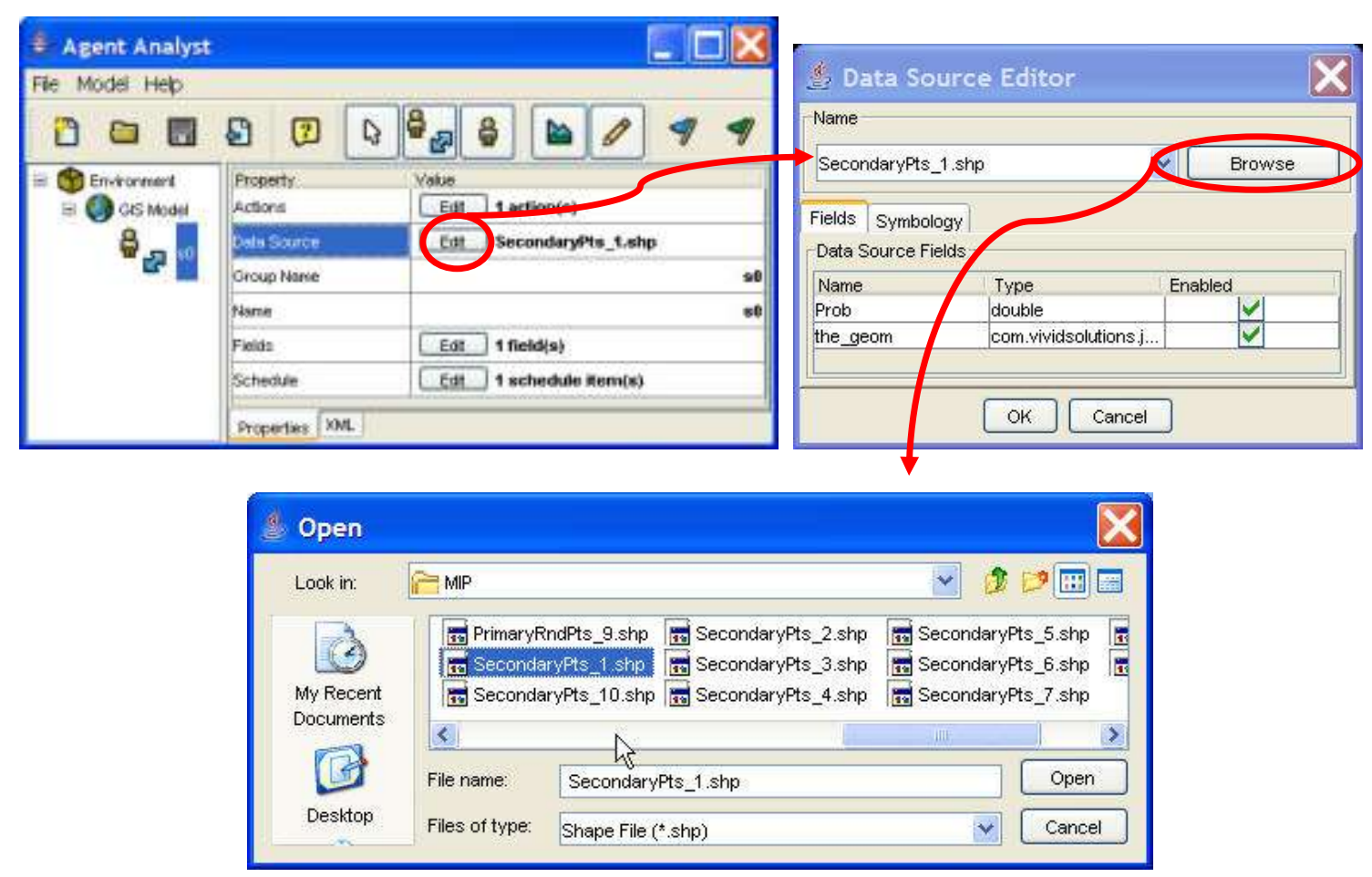

Figure 5.40. Vector Agent's Data Source Editor

The model also needs to take into account which dataset it will be editing. The writeAgents model action was modified to have the vector agents edit the linked shapefile (see Figure 5.41). In the case of the germination simulation, the agent's name is s0 and the pathname is ./projects/MIP/SecondaryRndPts_1.shp. As stated before, the shapefile needs to be in the project folder within the AA directory and this is why the pathname starts with a period.

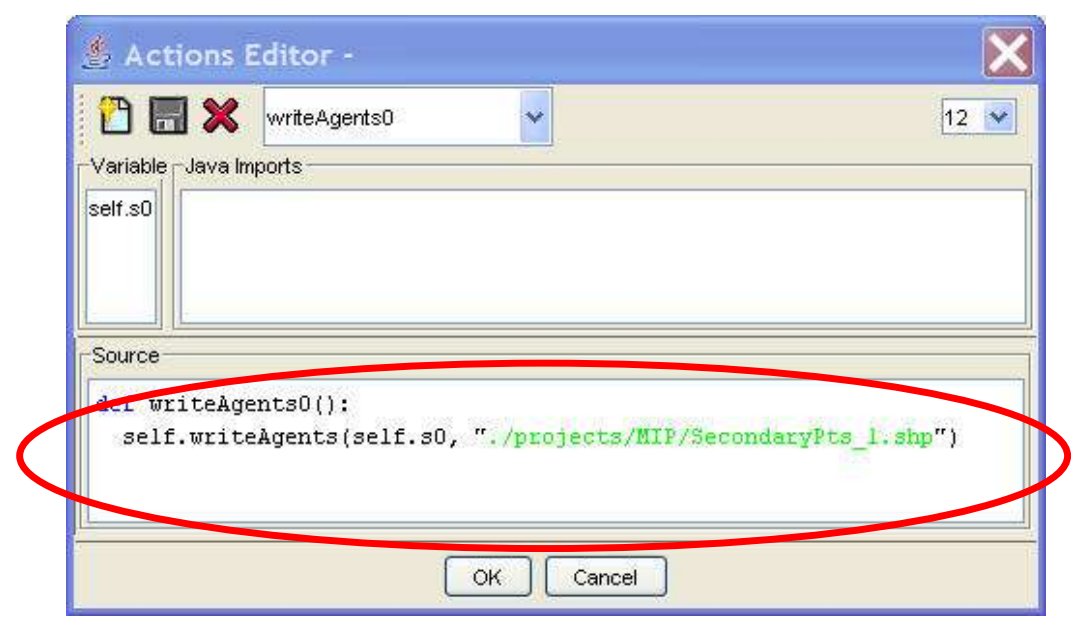

Figure 5.41. Model's Actions Editor 


\subsubsection{Seed Germination Simulation: Code for Agent Actions}

Whether an agent survives to germinate into an annual brome plant is determined by comparing a random number to the habitat probability. If a randomly generated number is less than the habitat probability, then the seed survives and there is no point position change. If a randomly generated number is more than the habitat probability, then the seed is terminated and the point is moved to a predetermined junk pile.

To program this, only one double precision numeric variable was dimensioned in the Field Editor. This variable is assigned a randomly generated number by implementing the Random.uniform.nextDouble() method (see Appendix L). The number is then compared to the probability attribute inherited from the secondary agent shapefile, the name of which is Prob. If the random number is more than the Prob value, then the $\mathrm{X}$ and $\mathrm{Y}$ coordinate variables are subtracted from themselves. This leaves the updated $\mathrm{X}$ and $\mathrm{Y}$ coordinate with a value of zero degrees north and zero degrees east in the WGS UTM $11 \mathrm{~N}$ projection. Less code is involved in moving an agent than deleting it entirely, and as it will be seen in the next section, a simple clip to erase the junk pile is just as effective.

\subsection{Post-Processing Results}

The updated secondary agents go through a series of geoprocesses to convert the data into a format similar to the initial 2001 Annual Brome Cover dataset, a raster dataset with percent cover values. To accomplish this, three general steps are taken (see Figure 5.42). First, the secondary agents are repolygonized, meaning they are converted into polygon format. The dataset is then cleaned. Unnecessary fields, field clarifications, and further calculations all make the dataset more user-friendly. Reclassifying is the last step. The values are converted into the raster format and set to a scale that is comparable to the original.

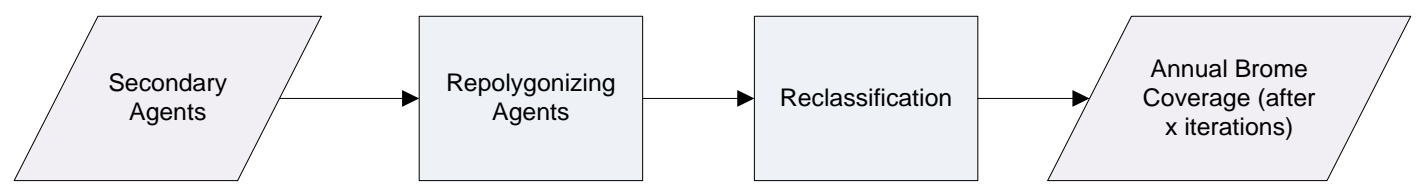

Figure 5.42. Post-Processing Results Workflow

\subsubsection{Repolygonizing Agents}

Repolygonizing agents from point form to polygon form has three steps (see Figure 5.43): the junk pile is removed from the dataset; the valid secondary agents are identified to what polygon they are contained by; a new attribute is calculated to help determine the raster values during the reclassification. 


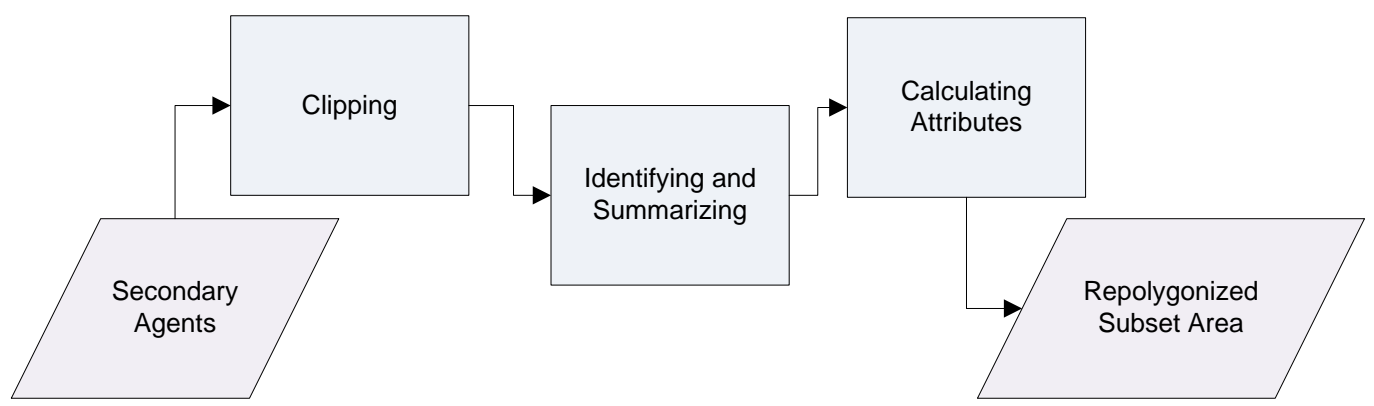

Figure 5.43. Repolygonizing Agents Workflow

The Germination Simulation left extraneous points at zero degrees east and zero degrees north. This junk pile is removed from the dataset by the Clip tool (see Appendix M). The subset area boundary, previously established by the user, is set as the clip feature. The remaining secondary agents are linked to the polygon in which they landed and germinated.

To identify where a secondary agent is, a Tally Value attribute field is added and calculated. This gives each secondary agent a value of one. The Identity tool is then run, attaching both the Tally Value and a polygon FID to each point in the attribute table. To determine how many agents are located in a specific polygon, the Summary Statistics tool is set to sum the Tally Value field per each polygon FID; the case field is set to the polygon FID.

A summary table indicates how many agents are present in each polygon. To recalculate the percent cover per polygon, the stand-alone summary table must first be linked to the subset polygon dataset by using the Add Join tool. The dataset then inherits the summary values and all the necessary attributes to calculate a percent cover. The last step is the recalculation. By taking the inverse of the equations used to calculate percent cover to seed density to seeds per polygon in Section 5.3.1, the summarized value can be converted into a percent cover (see Figure 5.44).

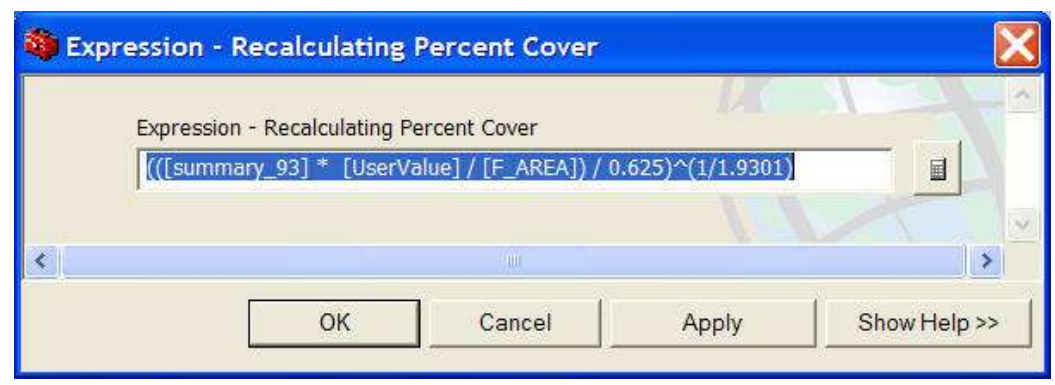

Figure 5.44. Expression the Calculates Percent Cover from Summary

\subsubsection{Reclassification}

The reclassification process involves converting polygons to the raster format, filling in spontaneously empty raster values, averaging the ten trials into one, and reclassifying the dataset to fit the percentage scale observed in the original dataset. The Feature to Raster tool is used to convert the polygons to raster format (see Appendix N). This tool was chosen over the Polygons to Raster tool because it produced fewer empty raster cells. 
However, to fill any amount of empty raster cells, a string of geoprocesses - the Block Statistics tool, Is Null tool, and Con tool - interpolates the missing data.

The Block Statistics tool first calculates the average three-by-three cell neighborhoods of values, producing a more generalized raster with a 90 by 90 meter cell size. The Is Null tool then gives values that had NoData an arbitrary value. Lastly, the Con tool is configured to find which cells have the arbitrary values, established by the Is Null tool, and gives those cells the interpolations.

This clean up of the raster datasets occurs ten times, one for each of the parallel simulations. The Cell Statistics tool was used to average the ten datasets together. This tool overlays all ten rasters and finds the average on a cell-by-cell basis.

The last step in the Reclassification model is the actual reclassification. The Slice tool was used to convert the averaged values from the previous step into a scale similar to the original dataset (see Figure 5.45). It divides the values into 41 classes at equal intervals. The resulting dataset then has values ranging from 0 to 40 , similar to that of the percent cover scale.

A presence and absence version was also created to complement the normalized dataset. The Reclassify tool took values 0 to 9 and 41 to 100, set them equal to zero, and took values 10 to 40 and set them equal to one. The result is a dataset where zero represents absence and one represents presence.

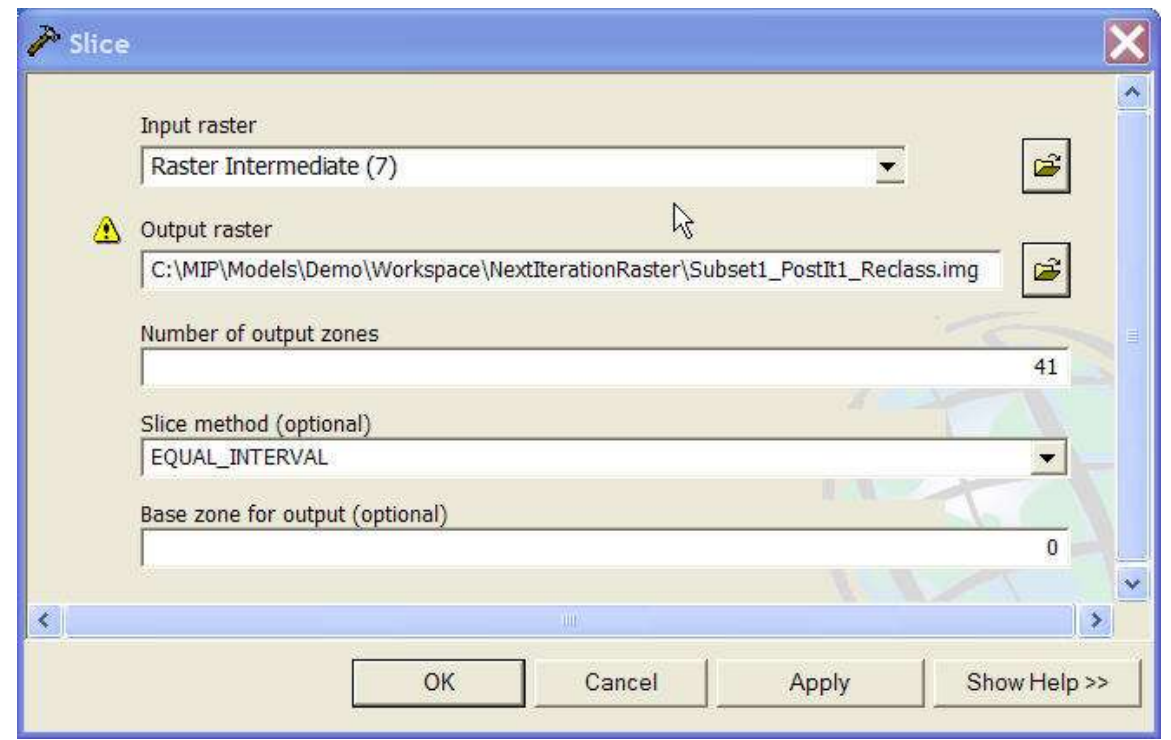

Figure 5.45. Slice Tool Parameters 



\section{Map User Interface}

Since some of the steps in the Data Manipulation tier of the system architecture involve user-delineated areas, a map user interface was created to help judge where these areas should be. A user may choose to use his or her map document or data for delineating by loading the Annual Brome ABMS toolbox. To prevent burdening users who lack such materials, a map document titled DemoMap organizes and clearly labels valuable data (see Figure 6.1; see Appendix F).

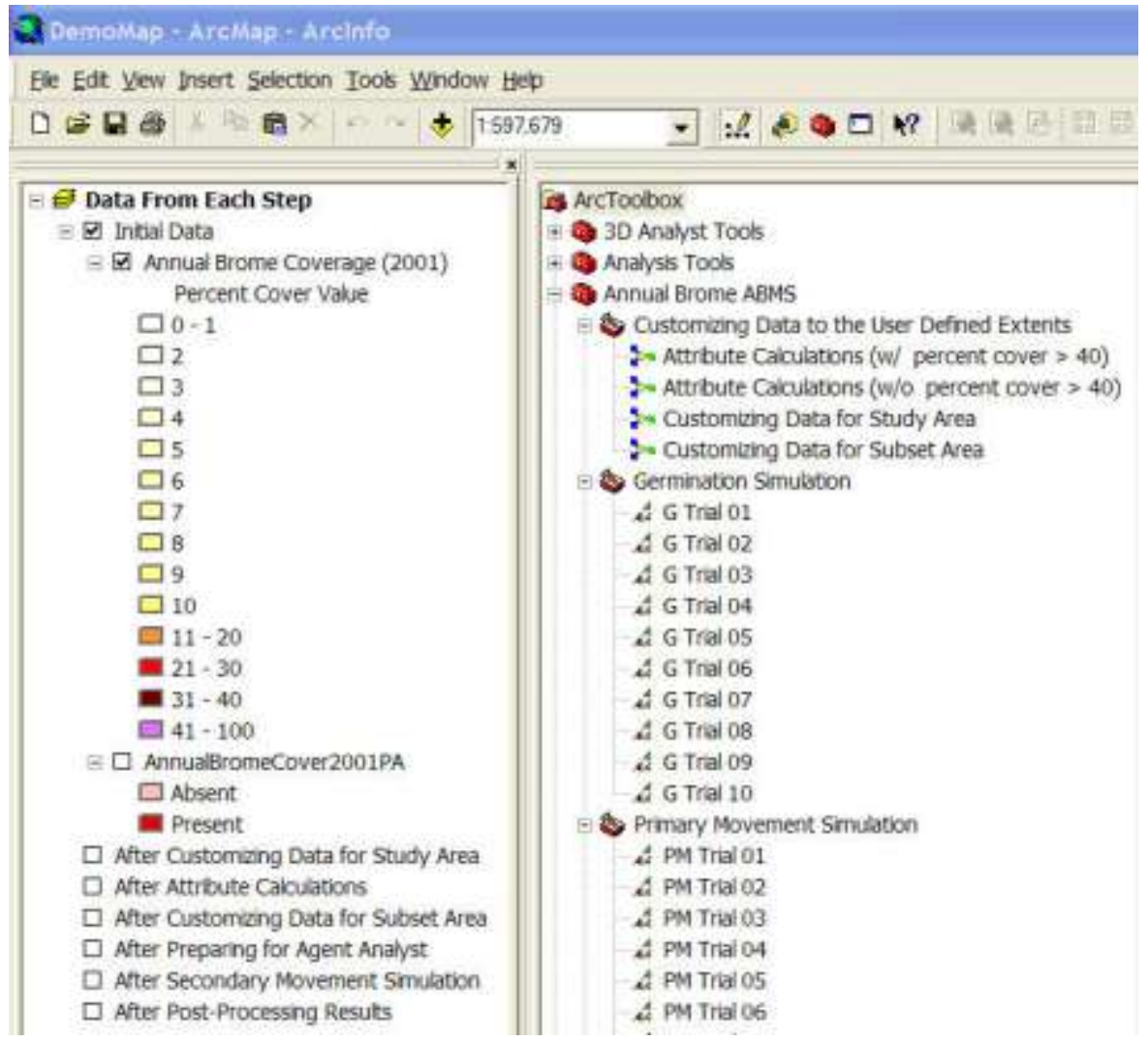

Figure 6.1. ArcMap's Table of Contents and ArcToolbox

In the table of contents (TOC), there are seven data groups. The only group occupied is the Initial Data, which contains a classified 2001 Annual Brome Cover and 2001 Annual Brome Cover for Presence/Absence with presence indicated by values between 10 and 40 and absence indicated by values between 0 and 9 and between 41 and 100. From these two datasets, the user can gain a general understanding as to where he or she would like to establish a study area. For more data, the user can Add Data from the provided Data folder (see Figure 6.2) 


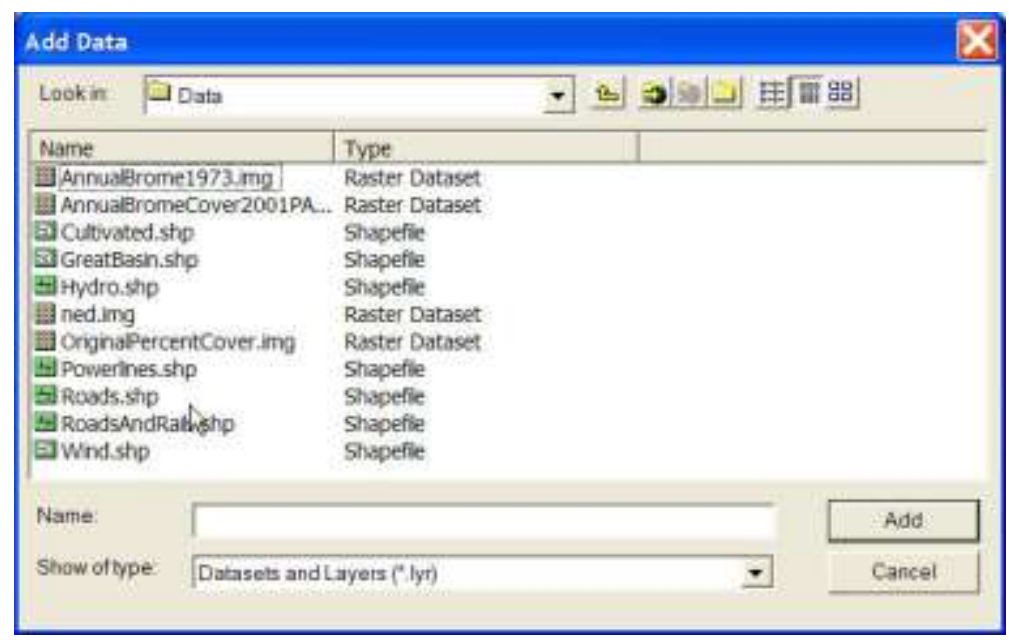

Figure 6.2. Add Data Window

Three color schemes were used to represent percent values (see Figure 6.3). A gradual darkening of yellow leading up to ten indicates the annual brome presence/absence cutoff fuzziness. An orange transitioning to dark red emphasizes a more exponential increase in cover for values 10 to 40 . The remaining values are colored purple to emphasize them as outliers.
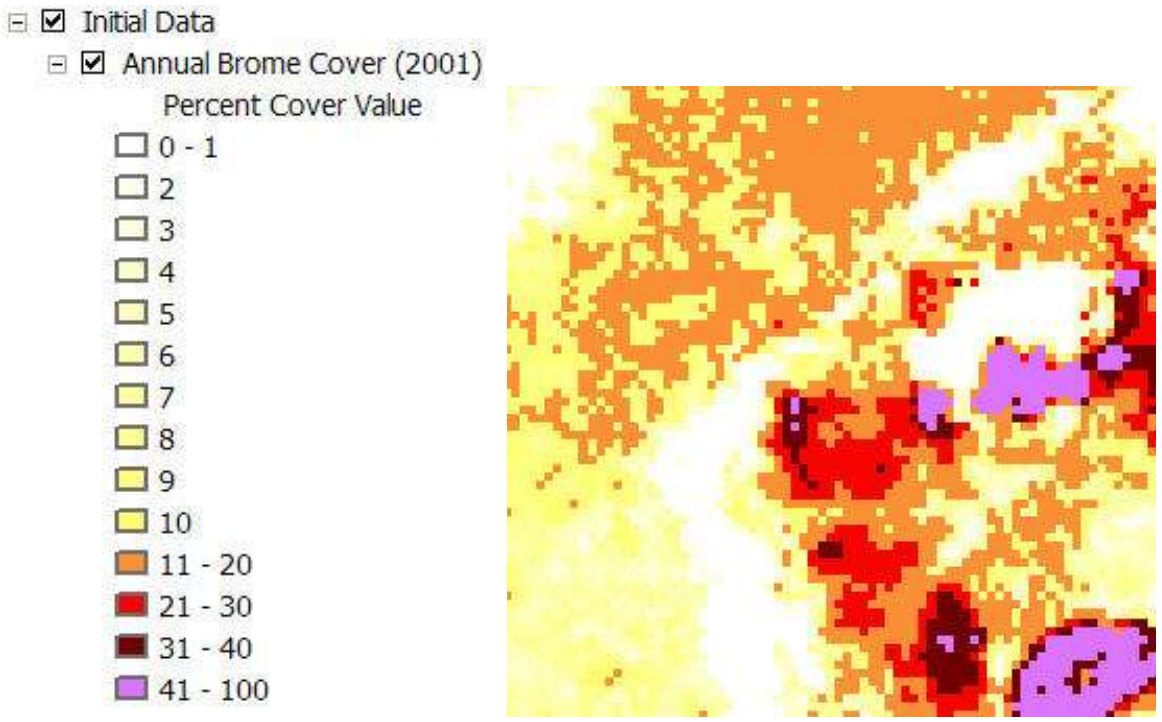

Figure 6.3. Symbolized Annual Brome Cover for 2001

In addition to the value classification, the 2001 Annual Brome Cover dataset is represented through presence/absence (see Figure 6.4). To make the symbolization as simple as possible, red was used with two levels of brightness. This lets the user distinguish the two categories while at the same time it is easy remember which symbolizes which. 


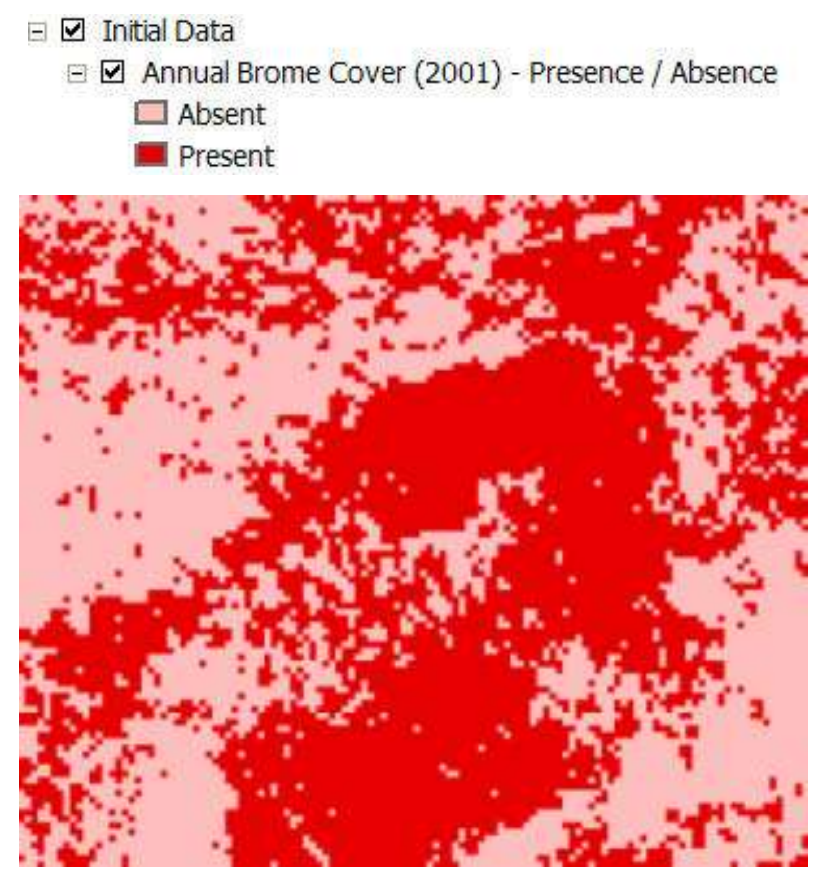

Figure 6.4. Symbolized Annual Brome Cover for 2001 - Presence/Absence

When the user is ready to start the agent-based simulation, he or she simply adds the Annual Brome ABMS toolbox to ArcToolbox and follows the steps described in sections 5.2-5.7. Within the toolbox, there are three toolsets: one important to customizing the data, and the other two for Agent Analyst.

The Customizing Data to the User Defined Extents toolset groups three distinct models, one of which has two versions. The user starts by opening the Customizing Data for the Study Area model. When the form opens, there are eight data input parameters, eight data output parameters, and one parameter for delineating the study area. The user does not need to change any of the inputs or outputs unless there is a white $\mathbf{X}$ within a red circle symbol, which indicates an invalid parameter setting (see Figure 6.5). There is also a black exclamation point within a yellow triangle symbol, indicating that the dataset can be overwritten; this parameter does not need to be changed unless the user does not want to overwrite.

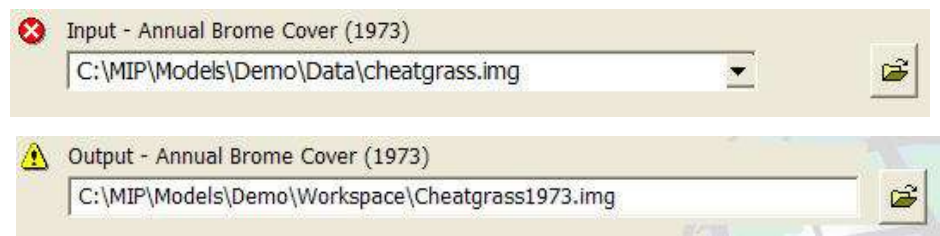

\section{Figure 6.5. Tool Symbols}

In order to delineate a study area, the user must create a feature set. A polygon feature set can be created by selecting the top button next to the parameter (see Figure 6.6). There is the option for creating more than one feature set, however it is recommended that only one be created per simulation. 


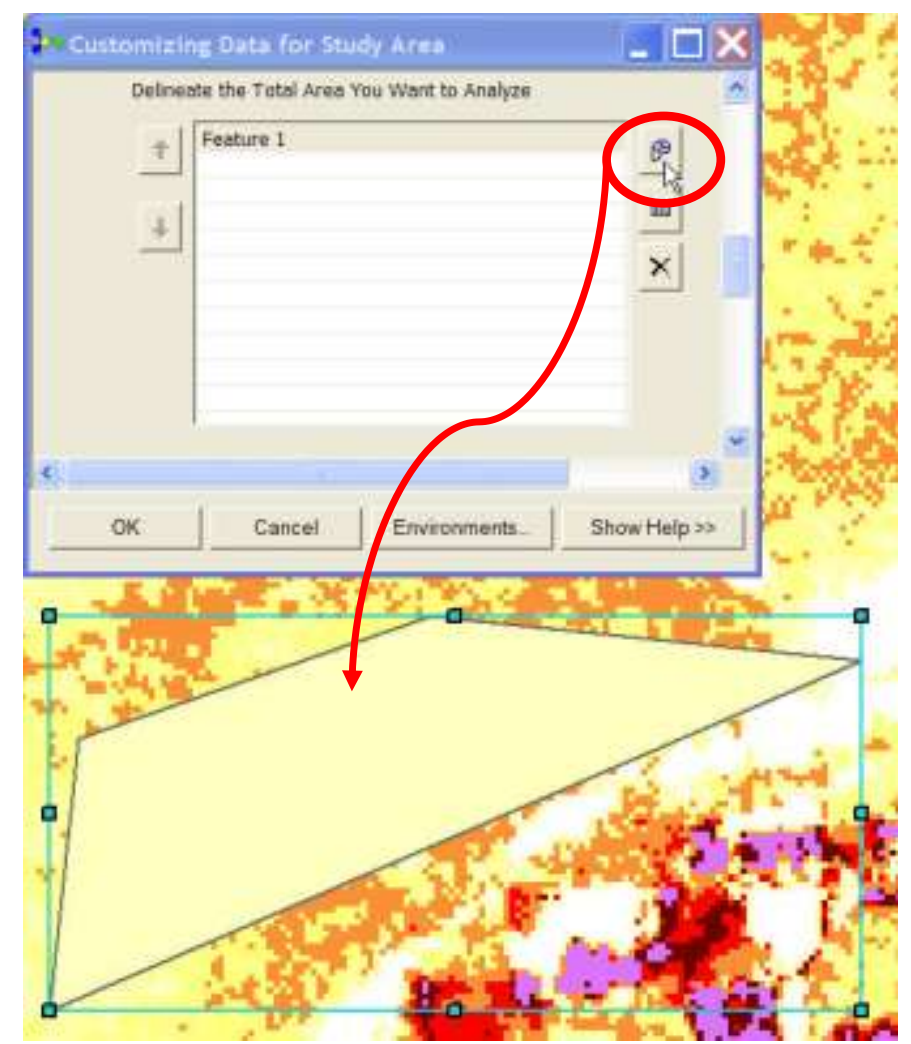

Figure 6.6. Delineating Areas

After the parameters are set and the model is run, the resulting data output is automatically added to the map display with pre-set symbology. There is a folder called Layers_for_Symbology that contains layer files that are imported for outputs' symbologies (see Figure 6.7).

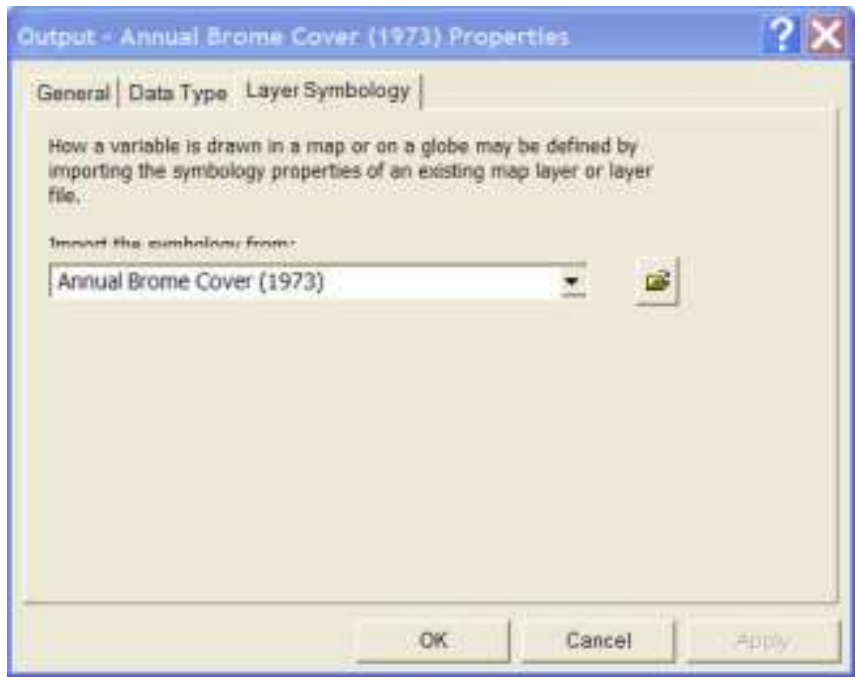

Figure 6.7. Importing Symbology for Tool Output

After each model is run, the output data layers appear at the top of the TOC. The user simply drags the new layers into the corresponding group. Group labels start with the word "after" followed by the name of the model just run (see Figure 6.8). By moving the 
layers to these groups, the user can keep track of which model steps have been run and view the output. Each data output, including the roads, railroads, etc. are symbolized with an imported layer. This makes it easy for the user to detect correlations with model output.

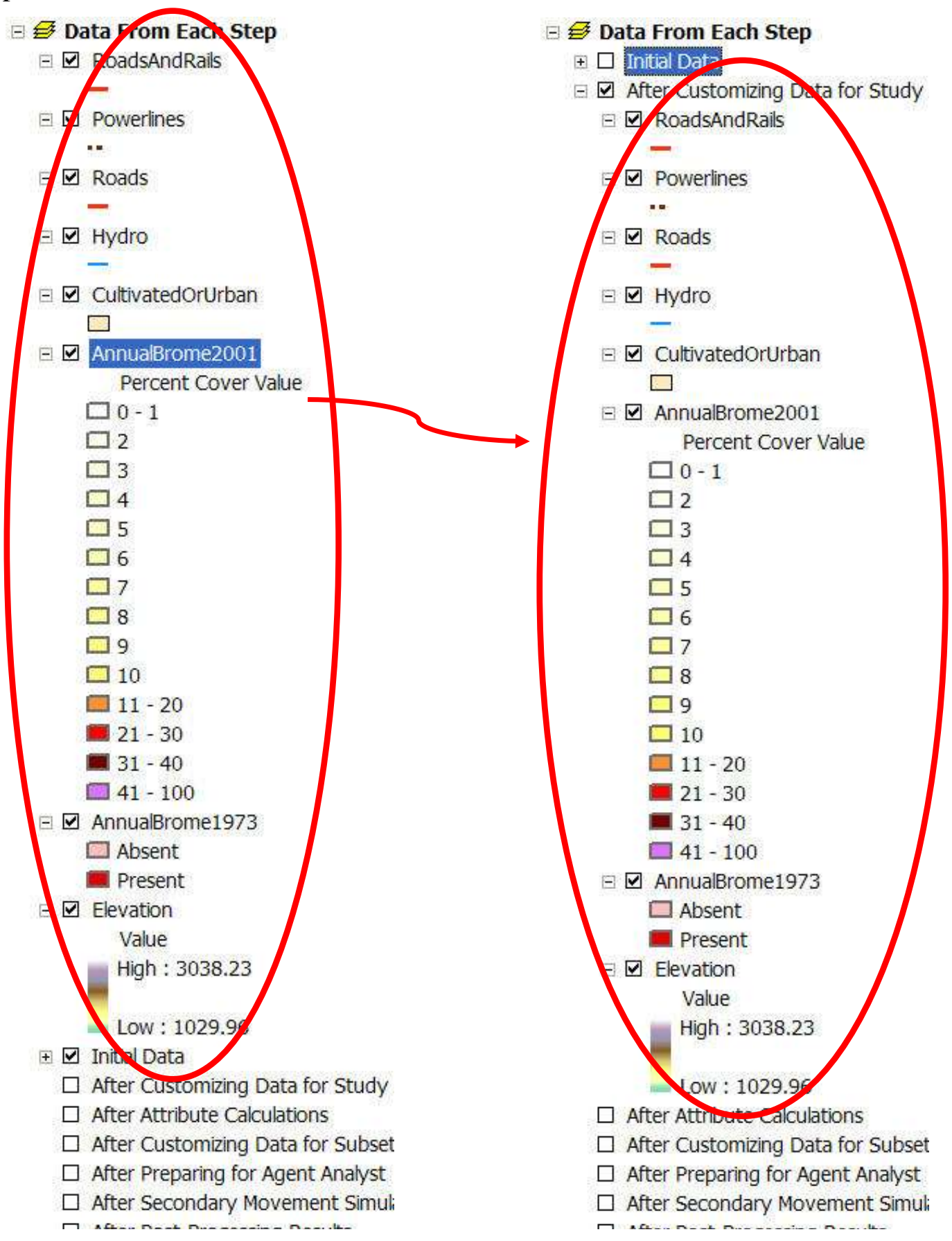

Figure 6.8. Rearranging the Table of Contents

The AA simulation steps are unique in comparison to the others. The data are edited directly, and as it would be useful to see the pre-simulation points in contrast to the postsimulation points, the user would have to make a copy of the pre-simulation data manually. It should also be noted that the post-simulation data will not appear in the map 
display until after a refresh; the user can see the movement once he or she hits the refresh button.

\subsection{Running Agent Analyst Tools}

To initiate the Primary Simulation or Germination Simulation steps in the model, the user will need to open the AA tools. The tools have been pre-set to activate the saved model file containing the model actions, agent actions, and shapefile-to-model link information. The user simply double clicks the tool in the corresponding toolset and runs it. AA will open along with the Repast Simulation toolbar (see Figure 6.9). To start the simulation, the user simply clicks the single tick icon symbolized by a play button with a vertical bar to the left. When the simulation is complete, the tick count will read "1.0".

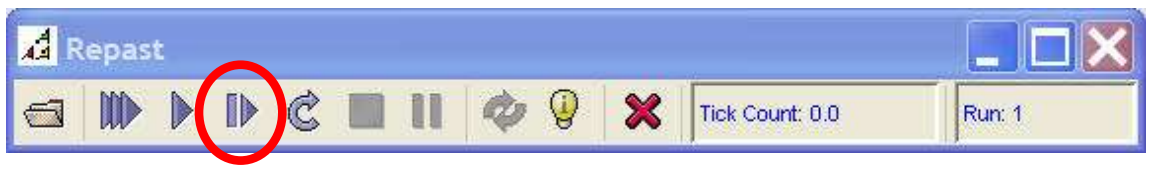

Figure 6.9. Initiate Agent Analyst Simulation Icon 


\section{Data Server}

The complexity of the agent-based model makes it difficult to implement over the web. As of now, the web application contains data for three runs of the agent-based model; these results are described in Section 9.4. These results, however, are representative of the model's early development. In the following sections, how the datasets were integrated in the geodatabase, comparing and contrasting data layers versus real-time geoprocessing, and modifications made to Armstrong's original base map will be discussed.

\subsection{Geodatabase Design}

The original geodatabase was compiled by Armstrong (2007). The projection was set to WGS 1984 UTM Zone 11N. The design of the geodatabase was simple, including raster datasets and feature classes. To keep this simplicity and add complementary data to this geodatabase, one feature class and five raster datasets were imported (see Figure 7.1).

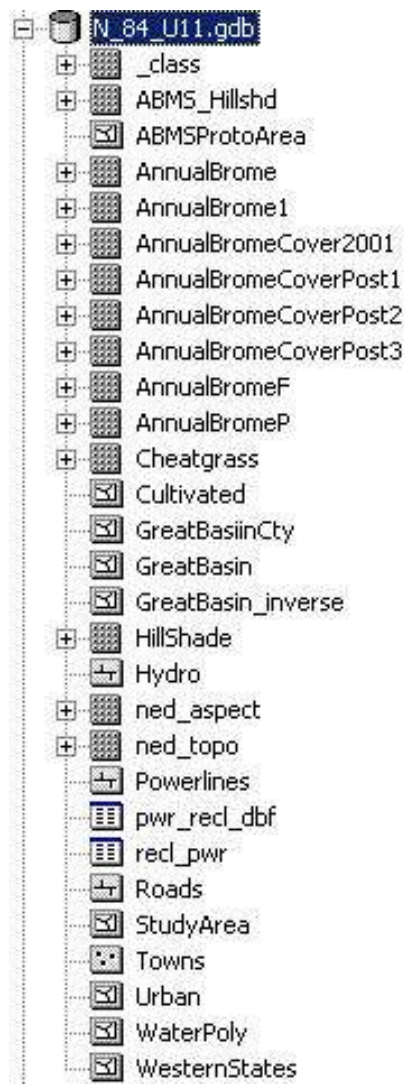

Figure 7.1. Data Server Geodatabase Design

The feature class, ABMSProtoArea, consists of a polygon boundary of the agent-based model's prototype area. This boundary is similar to Armstrong's StudyArea feature class, differing in its extent and symbology. One imported raster dataset, ABMS_Hillshd, is a hillshade surface analysis of the prototype area's digital elevation model (DEM). This raster is similar to Armstrong's Hillshade raster dataset that stretches across his entire study area. The remaining four imported rasters are unique to this project. They are the results of three iterations of the agent-based model. The word 
"post" in the name signifies the iteration that the data resulted from. For example, AnnualBromeCoverPost 2 was the result of the second iteration of the model. The AnnualBromeCover2001 is the original percent cover raster.

\subsection{Data Layer vs. Real-time Geoprocessing}

The matter of how to represent the agent-based model output is limited. Armstrong (2007) implemented the real-time geoprocessing concept. This is not only useful but also opens a direct interaction between the user and the results customized to the AOI. Unfortunately, because the agent-based model uses AA, a non-ESRI product, real-time geoprocessing is not possible. Instead, the results must be displayed in web application form as layers. As will be discussed in Chapter 11, if the model is constructed only in ModelBuilder, it could then be implemented in real-time.

\subsection{Modifications to Armstrong's Base Map}

The original annual brome map document provided by Armstrong was adapted. The changes, however, were minor and there were no alternations to the existing layers. The agent-based model prototype area extent was added, matching a similar symbology to Armstrong's study area; Armstrong created a simple texture with gray coloring, whereas the prototype area used the same texture with light yellow coloring. The same is true for the hillshade dataset. Instead of a gray hillshade tint used by Armstrong, a yellow tint was used to distinguish the prototype area.

\subsection{Web User Interface}

The web application modified from Armstrong (2007) has the same easy-to-use layout and main base map. The table of contents allows a user to turn layers on and off. In the new version, however, the agent-based model results are included. A layer group aggregates the results, giving the user easy access for activation and deactivation.

Navigation within the web application is simple. There are out-of-the-box zoom-and-pan tools, as well as a map overview window. At different zoom levels, and depending where the user is panned, scale dependencies turn layers and labels on and off at specified minimum and maximum map scales.

To enhance the visual appeal of the web application, a banner was created using three images. The blend tool in Adobe Photoshop created a fuzzy transition between the images of an annual brome-infested field, a fire, and the BASF logo. The banner emphasizes the importance of land management by calling attention to the flammability of annual brome.

For more information about the web application, refer to Appendix $\mathrm{O}$ and Armstrong (2007). 


\section{Testing}

Four sets of tests were conducted on the system. The first set was unit testing. As the model was constructed, observing what output resulted from the string of geoprocesses, whether the model would validate, and making sure each individual tool or script operated correctly were all extremely important during development. The second set led to the decision to conduct ten simulations in parallel for a single model run and then average them. The third set involved testing the web interface. Which representation was chosen for the agent-based model results and the options available will be discussed. The last set was how to determine the number agents in a simulation. It was important to know the consequences of generalization and how to mediate the problems.

\subsection{Unit Testing - Troubles with ArcGIS Desktop}

In the early stages of model development, the tedious nature of changing the output names every time the model was run became apparent. Schema locks were preventing unintentional overwriting. It was decided to reverse the default setting under the options menu (see Figure 8.1).

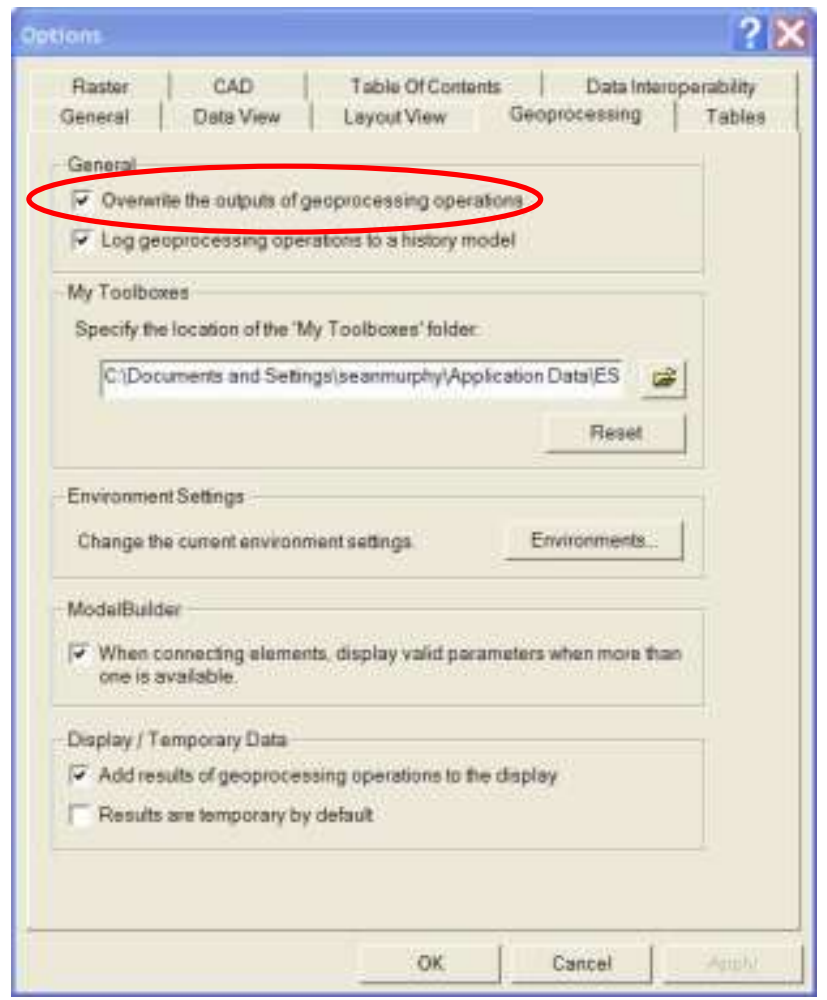

Figure 8.1. Setting Geoprocess to Overwrite Output

One category of schema lock that could not be reversed involved the Calculate Areas script. For unknown reasons, this script created errors ranging from redundant schema locks to total ArcMap failures. To avoid schema locks, it was found that ArcGIS Desktop had to be restarted to eliminate any inter-program connection. As for the spontaneous ArcMap failures, the model was recreated or a Feature Class to Feature Class tool converted the Calculate Areas output into a more flexible dataset. An example 
of the Feature Class to Feature Class tool can be seen in the Calculating Number of Agents section of the Preparing Data for Agent Analyst model (see Appendix E).

The Add Join tool was important for attribute inheritance among datasets but the flexibility is limited. Joins are intended to link Feature Layer to a table or feature class attribute table. This is ideal when editing directly in ArcMap, but not when creating models. To work around this limitation, two geoprocesses were added. The first is the Make Feature Layer tool. This takes a table or feature class and stores it in the computer's RAM, thus imitating a feature layer in the ArcMap's TOC. The new feature layer can then be joined with any other table or feature dataset. The output, however, is in the feature layer format, stored within the RAM. The second addition, the Copy Features tool, can then convert it into a feature class, a format stored on the hard disk.

After creating random points using the Create Random Points tool, the editing of that new shapefile was found to be limited. Clips, other Analysis Tools, and AA did not always work. An error message would indicate problems with geometry. To work around this issue, the file produced from the tool was converted into another dataset using the Feature Class to Feature Class tool. This new file was both editable and workable in AA.

The Clip tool designed for raster datasets did not operate correctly as far as clipping a raster to the extent of a feature class. Normally, the feature class would be the clip feature and the raster the input feature. This malfunctioned; the resulting raster was the same size as the input. By using the Copy Raster tool with an extent environment setting set to the feature class, the clip operation worked correctly.

The Clip tool designed for vector datasets also had some issues. After the dataset had undergone a simulation in AA, the clip tool would not operate. An error message would reveal a problem with point geometry. Instead of repairing the geometry, the dataset was copied using the Feature Class to Feature Class tool, placing a new shapefile in a folder outside of the AA directory. The new file automatically updated the geometry and the clipped tool operated correctly.

The Weighted Overlay tool used in the Habitat Suitability model also had some issues. Although Armstrong (2007) built the original model, when modified or validated, there were sometimes errors. The percent influence, set in the parameters, must equal 100. On occasion, when validating the tool, it would assume the total influence changed despite no modifications. Once the percentages were re-entered manually, the tool recognized that it was actually correct.

Another issue with the Weighted Overlay tool was the inflexibility. It was originally thought that the Habitat Suitability model could be run on smaller spatial extents. When an input was empty, however, the tool would not function. For each feature that had a percent influence, it needed some data included in the overlay, meaning larger spatial extents were needed to include at least a portion of each dataset.

The raster file format, in general, also had issues. First, naming files in the GRID format only accepts 13 characters. This limited the descriptive nature of the name. In instances where this was an issue, the raster output was changed to the imagine format, *.img, which allows more characters in the name. Second, when Map Algebra would calculate 
raster values in the floating point number format, it needed to be converted to integer before any reclassifications. This is the reason why there are multiple Integer tools in portions of the Calculating Attributes model. Lastly, converting rasters to the vector format requires cells to have data. This was an inconvenience during conditional statements that resulted in all cells being removed from the dataset. When there were no cells to be converted, the Raster to Polygon tool would not complete, but rather would stop and display an error message. To work around the tool's deficiencies, two versions were created for the Attribute Calculations model: one when there are values over 40 and one when there is not.

The most common error that occurred throughout the model development was in relation to the computer's RAM. When polygon datasets were too large to implement overlay functions, an error would indicate that there was insufficient RAM. This also occurred when AA had too many agents to edit. These two issues led to the incorporation of user defined extents and the acceptable range of agents (between 1 and 80,000). By having the user select areas of interest, the model frees up RAM that would otherwise be occupied, and it reduces computation time. It also allows for users to judge whether model run-time or accuracy is more important.

\subsection{Ten Iterations in Parallel}

Because this model is stochastic, values can change in one location with two exclusive runs of the model. To limit the outlier areas from representing a false percent cover, an average was taken from ten runs. The ten run guideline was determined by taking the maximum raw value after averaging 1 to 10 runs. As more runs were averaged together, an overall pattern showed a decrease in probability that the maximum value was an outlier. A plateau formed after six to seven runs (see Figure 8.2). This plateau indicates that by averaging more than seven runs, the most accurate final percent cover values are produced.

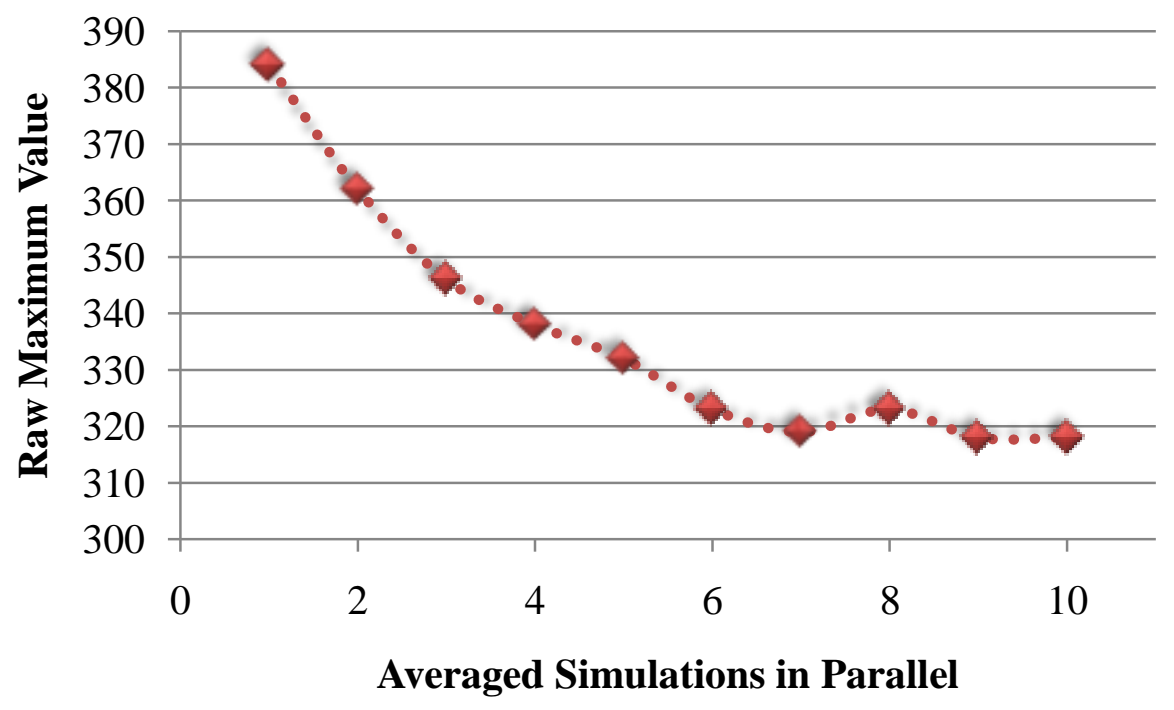

Figure 8.2. Correlation between Averaged Simulations in Parallel and Raw Maximum Value 


\subsection{Web Interface}

In the early development stages of the web interface, an AOI delineating tool was thought to be the best way for a land manager to access data. When the data are layers, however, and nothing is being geoprocessed, this extra step seemed illogical. Even though it would be useful to have a custom dataset return from the server to the web interface, it is simpler for a user to click a layer on and off. With this in mind, the AOI delineating tool for the agent-based model datasets was not included in the final web application.

\subsection{Generalization}

Because of the limitations in computer RAM and processing power, the number of agents in a single simulation needed an upper limit. Two approaches were tested and documented and a third solution was developed to the conceptual stage.

Before the tested solutions will be discussed, the problem will be clarified. There are two distinct consequences resulting from agent overload - too many agents for the computer's RAM and processor - when comparing ArcGIS Desktop and AA. ArcGIS Desktop doesn't seem to exhibit agent overload to the extent of model run-time failure; there were no instances of crashing because of too many agents. It does, however, have an significant increase in processing time, for each step, when there is an increase in the number of agents.

Agent Analyst, however, does have a threshold in which there is a model run-time failure. To prevent this, two different configurations of the model resulted in two different upper limits, each having positive and negative traits. First, when one vector agent group (in AA) is created for the entire point shapefile dataset, the upper limit is 80 thousand; the agent group cannot have more than 80 thousand agents because of agent overload; 80 thousand or less agents do not cause an overload.

Another approach is to split the dataset linked to Agent Analyst into multiple parts, each of which is assigned to a unique vector agent group. This allows AA to run the model on an agent-group-by-agent-group basis, freeing an amount of RAM per agent group and therefore raising the threshold. After testing this method, the upper limit was placed at 250 thousand. This means that the agent sum of all vector agent groups could not exceed 250 thousand without model failure. The main drawback to this approach is a significantly greater processing time involved in partitioning the point datasets using the Split tool, which can range from 10 minutes to over an hour per trial.

\subsubsection{Ensuring No Agent Overload}

To prevent agent overload, a methodology to control the number of produced agents was necessary. The 250 thousand method required a more in-depth calculation, requiring the user to use Microsoft Excel. This approach was used early on during model development but was soon replaced by a method that was less cumbersome - the 80 thousand method.

For the 250 thousand value, it was thought that the study area could be divided into equal areas, the total number of seeds calculated for each, and then the section with the most seeds would set a maximum. This maximum would then be the basis for a division factor: a simple ratio between the maximum over 250 thousand. 


$$
\text { Divison Factor }=\frac{\text { Maximum }}{250,000}
$$

Each polygon in the subset could then be divided by this factor to establish the number of agents per polygon; no section would exceed that 250 thousand agent threshold.

Although having 250 thousand agents may have been more accurate in the long-term, it was impractical with the run-time ranging from hours to days for each step. As a substitution, the 80 thousand methodology was implemented. This method improves model time and efficiency in a few regards. First, the Split tool was no longer needed to partition datasets for AA. This cut geoprocessing times significantly when preparing the data. Second, the amount of user-AA interaction was reduced because only one vector agent group was needed to be run per simulation tick instead of four. This reduces the user error during AA's direct editing and the time the user needs to spend in a foreign interface. Lastly, the user can interact with the data preparation process through area delineation to customize the output to a specific AOI, thus increasing output efficacy.

As discussed in Sections 5.2.2 and 5.2.4, the user delineates two areas. The first is the extent of the entire study area which is used in calculating and producing a division factor. The second is the extent of a subset area in which the division factor is applied and on which the actual model is run. This was done to significantly reduce processing times for areas that land managers may find more important within the study area; to analyze the entire study area at once, the user simply delineates a subset of the entire study area.

The 250 thousand and 80 thousand methods were not without glitches. In the number of agents calculation, there were rounding issues where polygons with few seeds were excluded (see Figure 8.3). This is because when the division factor was applied to each polygon, if the seed value was small, then the division factor would result in a fractional agent. If the fraction was below 0.5, the polygon would not receive an agent. This issue significantly impacted the results since a large portion of the dataset had low seed values. 


\begin{tabular}{|c|c|c|c|c|c|c|c|c|c|}
\hline \multicolumn{9}{|c|}{ 国 Attribuzes of allicolygonvaluma forabrect } & \multirow[b]{2}{*}{ NoseedAgnt } \\
\hline & SeedPerlM & SeedinPoly & & F AREA & Totalseeds & TotSeedVal & UserDetAgt & Divfactor & \\
\hline & 38705 & 31438136.2494 & & 812.25 & 3146 & 241199981 & 10000 & 24119999 & 0.1303 \\
\hline & 36686 & 31422703.5029 & & 81225 & 3144 & 241199981 & 10000 & 2411999 & 0.1303 \\
\hline & 38705 & 314381362519 & & 81225 & 3144 & 241199981 & 10000 & $2411999 / 1$ & 0.1303 \\
\hline & 38728 & $12582727+99$ & & 81225 & 3146 & 241199981 & 10000 & 24119981 & 0.5217 \\
\hline & 38705 & 157190681255 & & 812.25 & 3146 & 241199981 & 10000 & 24119981 & 06517 \\
\hline & 28967 & 23520323.253 & & 4061.25 & 15728 & 241199981 & 10000 & 24119961 & 0.0975 \\
\hline & 38686 & 31422703.5015 & & 4061.25 & 15728 & 241199981 & 10000 & 241199981 & 0.1303 \\
\hline & 38686 & 314227035015 & & 81225 & 3144 & 241199981 & 10000 & 24119981 & 0.1303 \\
\hline & 38728 & 31456818.004 & & 1624.5 & 6295 & 241199981 & 10000 & 24119981 & 0.1304 \\
\hline & 38753 & 31477124.2539 & & 1624.5 & 6295 & 241199981 & 10000 & 24119981 & 0.1305 \\
\hline & 38753 & 31477124254 & & 1055925 & 40894 & 241199981 & 10000 & 24119981 & 0.1305 \\
\hline & 38705 & 31438136.2515 & & 10559.25 & 40894 & 241199981 & 10000 & 24119981 & 0.1303 \\
\hline & 38753 & 31477124.254 & & 10559.25 & 40894 & 241199981 & 10000 & 24119981 & 0.1305 \\
\hline & 38705 & 31438136254 & & 81225 & 3148 & 241199981 & 10000 & 24119981 & 0.1303 \\
\hline & 38728 & 314568818004 & & 1624.5 & 6295 & 241199981 & 10000 & 24119981 & 0.1304 \\
\hline & 38728 & 31456818,0039 & & 16245 & 6295 & 241199981 & 10000 & 24119981 & 0.1304 \\
\hline & 38705 & 314381362515 & & 812.25 & 3142 & 241199981 & 10000 & 24119981 & 0.1303 \\
\hline & 38728 & 314568818.0039 & & 812.25 & 3142 & 241199981 & 10000 & 24119981 & 0.1304 \\
\hline & 38728 & 31456818.0015 & & 1055925 & 40870 & 241199981 & 10000 & 24119981 & 0.1304 \\
\hline & 38728 & 31456818004 & & 1055925 & 40870 & 241199981 & 10000 & 24119981 & 0.1304 \\
\hline & 38728 & 314568180015 & & 81225 & 3144 & 241199981 & 10000 & 241199981 & 0.1304 \\
\hline & 38705 & 31438136.2539 & & 81225 & 31423 & 241199981 & 10000 & 24119981 & 0.1303 \\
\hline & 38728 & 314568180019 & & 81225 & 3146 & 241199981 & 10000 & 2411998 & 0.1304 \\
\hline & 38686 & 31422703.5019 & & 1624.5 & 6285 & 241199981 & 10000 & 2411999 & 0.1303 \\
\hline & 38705 & 220066953.749 & & 812.26 & 3146 & 241199981 & 10000 & 2411999 & 0.9124 \\
\hline & 38728 & 629135359961 & & 16245 & 5285 & 241199981 & 10000 & 24119998 & 0.2608 \\
\hline & 39686 & 188536221.004 & & 81226 & 3146 & 241199981 & 10000 & 24119998 & 0.7817 \\
\hline & 38705 & $6287627 ? 5054$ & & 242675 & 94,37 & $3 \$ 1199981$ & 100 ก & 241199081 & $\cap>\pi \cap 7$ \\
\hline \multirow[t]{2}{*}{ 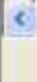 } & & & & & & & & & \\
\hline & Record: 14 & 0, & $n$ & Shaw: $\sqrt{\text { All }}$ & Selected & \multicolumn{2}{|c|}{ Records ( 4 out of 110265 Selected) } & Options - & \\
\hline
\end{tabular}

Figure 8.3. Attribute Table with Values That Result in Agents Highlighted

\subsubsection{Initial Three Solutions}

To mediate this problem, three solutions were devised, one of which has reached the conceptual stage. The first involves editing the raster dataset before the Attribute Calculations model is implemented. By editing the size of cells with lower seed densities, the area and therefore the total number of seeds per cell would increase. This could be implemented using the Block Statistics tool to average a certain dimension of cells together, resulting in a more significant number of seeds. After the conversion from raster to polygon formats, the polygons would be larger but would have a similar amount of seeds in comparison to the smaller but higher seed density polygons.

By combining all fractional values together, a certain bias is ignored. The first solution, for example, combines two cells with a value of 0.05 and 0.49 , doubling the area. The twice as large cell now has a value of 0.54 and would receive a randomly placed agent. Since the 0.05 and the 0.49 parts of the cell have an equal chance of receiving that agent, the bias influence, which normally gives the larger value a higher chance of receiving an agent, is eliminated.

The second solution would be to change the rounding point from 0.5 to a lesser value; a rounding point of 0.3 will be used as an example. This is done simply by editing the attribute table before the agents are randomly placed within the polygons. First the polygons that have a number of agents less than 0.5 but more than 0.3 are selected. A value of 0.2 is then added to those selected values, manipulating the true number. This increases the fraction so that a random agent is placed in that polygon. 
There are two drawbacks to this solution. First, it is an iterative process. A new rounding point of 0.3 may not suit the user-defined number of agents, but rather a higher or lower value would produce a total number of agents closer to that particular amount. The method of trial and error would have to be used to find this ideal rounding point. Second, by changing the rounding point from 0.5 to a lower value, values between 0.5 and the new rounding point are treated the same as values between 0.5 and 1.49 . This expands the interval in which one agent is randomly created but does not equally increase the intervals in which two agents (1.5 to 2.49), three agents (2.5 to 3.49), etc. are randomly created.

The third solution involves the development of another geoprocessing string. After the number of agents field is calculated for each polygon, all the fractional agents less than 0.5 are selected and added together, resulting in a sum value. The polygons that have fractional values are then dissolved together to form a multi-part polygon. Then, within the constraints of this polygon, the fractional value's sum determines the number of additional agents to be randomly placed.

As in solution I, the bias distribution problem is evident. All areas of the multi-part polygon have an equal chance of receiving a randomly placed agent, even though certain sections may have contributed 0.49 to the fractional value sum and certain sections may have contributed 0.05 .

The three solutions all have a common issue in addition to their exclusive ones. Since the frequency distribution is skewed towards the lower values, there is overestimation in the total number of agents when any of the three solutions are applied. This overestimation is caused by the intermittent values between 0.01 and 0.99 ; between 1.01 and 1.99 ; between 2.01 and 2.99; etc. To give values lower than 0.5 an agent rather than exclude them would create large agent influx as opposed to just replacing the excluded ones. This is because when no solution is applied, the lower intermittent values (ie. between 0.01 and 0.049) would typically be rounded down and the higher intermittent values (ie. between 0.51 and 0.99 ) would typically be rounded up. When a solution is applied, the rounding down of values lower than 0.5 is ignored causing the skewed frequency distribution to overcompensate for fractional values. 



\section{Discussion}

The discussion chapter is broken into four sections: the first strictly describes the data outputs. The next two sections investigate the results of an $11 \mathrm{~km}$ by $12.5 \mathrm{~km}$ test area (137.5 sq km): the first uses 80 thousand agents and the second uses 10 thousand agents. The last section reviews the results when applying a 250 thousand agent maximum to a prototype area section of $10.5 \mathrm{~km}$ by $14 \mathrm{~km}(147 \mathrm{sq} \mathrm{km})$.

\subsection{Data Output}

The current model produces 21 datasets (see Table 9.1). These outputs are placed within the workspace folder and within the AA directory depending on how they were used later in the model. For datasets representing vector features, they are in the shapefile (*.shp) format, and for datasets representing continuous fields, they are in the Imagine (*.img) format. 
Table 9.1. Data Output

\begin{tabular}{|c|c|c|c|}
\hline Name & Feature & Format & Location \\
\hline AnnualBrome1973 & Annual brome cover in 1973 & Imagine & Workspace \\
\hline AnnualBrome2001 & Annual brome cover in 2001 & Imagine & Workspace \\
\hline CultivatedOrUrban & Cultivated/Urban Areas & Shapefile & Workspace \\
\hline Elevation & Elevation & Imagine & Workspace \\
\hline Hydro & Hydrology & Shapefile & Workspace \\
\hline Powerlines & Major Power Lines & Shapefile & Workspace \\
\hline Roads & Roads & Shapefile & Workspace \\
\hline RoadsAndRails & Roads and Railroads & Shapefile & Workspace \\
\hline HabitatProbabilities & Probabilities of germination & Shapefile & Workspace \\
\hline SeedDensity & Seed Density & Shapefile & Workspace \\
\hline PlantDensity & Plant Density & Shapefile & Workspace \\
\hline Outliers_Polygons & Outlier Areas & Shapefile & Workspace \\
\hline SubsetPercentCover2001 & $\begin{array}{l}\text { Annual brome cover in } 2001 \\
\text { for the subset }\end{array}$ & Imagine & Workspace \\
\hline $\begin{array}{l}\text { All_polygonvalues_ } \\
\text { forsubset1 }\end{array}$ & $\begin{array}{l}\text { Subset Area with Attribute } \\
\text { Values }\end{array}$ & Shapefile & Workspace \\
\hline $\begin{array}{l}\text { All_polygonvalues_ } \\
\text { wArea }\end{array}$ & $\begin{array}{l}\text { Subset Area with Attribute } \\
\text { Values Plus Area }\end{array}$ & Shapefile & Workspace \\
\hline $\begin{array}{l}\text { All_polygonvalues_ } \\
\text { forsubset2 }\end{array}$ & $\begin{array}{l}\text { Subset Area with Attribute } \\
\text { Values Plus Number of Agents }\end{array}$ & Shapefile & Workspace \\
\hline PrimaryRndPts_* & Agents for Primary Movement & Shapefile & $\begin{array}{l}\text { AA } \\
\text { Directory }\end{array}$ \\
\hline SecondaryPts_* & $\begin{array}{l}\text { Agents for the Germination } \\
\text { Simulation }\end{array}$ & Shapefile & $\begin{array}{l}\text { AA } \\
\text { Directory }\end{array}$ \\
\hline Subset**_Postit***_Raw & $\begin{array}{l}\text { Raw output of the Agent-based } \\
\text { Model }\end{array}$ & Imagine & Workspace \\
\hline Subset**_Postit***_NextIter & $\begin{array}{l}\text { Classified output of the Agent- } \\
\text { based Model }\end{array}$ & Imagine & Workspace \\
\hline Subset**_Postit ${ }^{* * *}$ _PA & $\begin{array}{l}\text { Presence/Absence output of the } \\
\text { Agent-based Model }\end{array}$ & Imagine & Workspace \\
\hline
\end{tabular}

$*$ = trial number

$* *=$ subset number (if multiple subsets in one study area)

$* * *$ = iteration number

The outputs are generated from different steps in the agent-based model. The first eight datasets are the result of Customizing Data for the Study Area: AnnualBrome1973, AnnualBrome2001, CultivatedOrUrban, Elevation, Hydro, Powerlines, Roads, and RoadsAndRails (see Table 9.2). With the exceptions of AnnualBrome2001 and Roads, all the datasets are used in the Habitat Suitability Model. AnnualBrome2001 is used later in the Seed Density Calculation and Plant Density Calculation. Roads is used later in the Secondary Movement Simulation. 
Table 9.2. Dataset Description and Importance

\begin{tabular}{|c|c|c|}
\hline Name & Description & Importance \\
\hline AnnualBrome1973 & $\begin{array}{l}\text { Clipped to the user's } \\
\text { study area }\end{array}$ & $\begin{array}{l}\text { Used in calculating habitat } \\
\text { suitability }\end{array}$ \\
\hline AnnualBrome2001 & $\begin{array}{l}\text { Clipped to the user's } \\
\text { study area }\end{array}$ & $\begin{array}{l}\text { Used in calculating attribute } \\
\text { values }\end{array}$ \\
\hline CultivatedOrUrban & $\begin{array}{l}\text { Clipped to the user's } \\
\text { study area }\end{array}$ & $\begin{array}{l}\text { Used in calculating habitat } \\
\text { suitability }\end{array}$ \\
\hline Elevation & $\begin{array}{l}\text { Clipped to the user's } \\
\text { study area }\end{array}$ & $\begin{array}{l}\text { Used in calculating habitat } \\
\text { suitability }\end{array}$ \\
\hline Hydro & $\begin{array}{l}\text { Clipped to the user's } \\
\text { study area }\end{array}$ & $\begin{array}{l}\text { Used in calculating habitat } \\
\text { suitability }\end{array}$ \\
\hline Powerlines & $\begin{array}{l}\text { Clipped to the user's } \\
\text { study area }\end{array}$ & $\begin{array}{l}\text { Used in calculating habitat } \\
\text { suitability }\end{array}$ \\
\hline Roads & $\begin{array}{l}\text { Clipped to the user's } \\
\text { study area }\end{array}$ & $\begin{array}{l}\text { Used in the Secondary } \\
\text { Movement Simulation }\end{array}$ \\
\hline RoadsAndRails & $\begin{array}{l}\text { Clipped to the user's } \\
\text { study area }\end{array}$ & $\begin{array}{l}\text { Used in calculating habitat } \\
\text { suitability }\end{array}$ \\
\hline HabitatProbabilities & $\begin{array}{l}\text { Germination probabilities } \\
\text { calculated from the } \\
\text { habitat suitability model }\end{array}$ & $\begin{array}{l}\text { A variable that determines agent } \\
\text { survivorship }\end{array}$ \\
\hline SeedDensity & $\begin{array}{l}\text { Seed density values } \\
\text { calculated from } \\
\text { regression equations (see } \\
\text { Section } 5.2 .3 .2 \text { ) }\end{array}$ & $\begin{array}{l}\text { A variable in calculating the } \\
\text { number of agents per simulation }\end{array}$ \\
\hline PlantDensity & $\begin{array}{l}\text { Plant density values } \\
\text { calculated from } \\
\text { regression equations (see } \\
\text { Section 5.2.3.3) }\end{array}$ & $\begin{array}{l}\text { A variable in calculating an } \\
\text { agent's travel distance during the } \\
\text { Primary Movement Simulation }\end{array}$ \\
\hline Outliers_Polygons & User delineated outliers & Excluded areas \\
\hline SubsetPercentCover2001 & $\begin{array}{l}\text { Annual brome cover for } \\
2001 \text { clipped to the user's } \\
\text { subset area }\end{array}$ & $\begin{array}{l}\text { User can compare this to the } \\
\text { simulation results }\end{array}$ \\
\hline $\begin{array}{l}\text { All_polygonvalues_ } \\
\text { forsubset } 1\end{array}$ & $\begin{array}{l}\text { All calculated attributes } \\
\text { after Customizing Data to } \\
\text { the User Defined Extent } \\
\text { (Section 5.2.1) }\end{array}$ & $\begin{array}{l}\text { Used by Preparing for Agent } \\
\text { Analyst }\end{array}$ \\
\hline $\begin{array}{l}\text { All_polygonvalues_ } \\
\text { wArea }\end{array}$ & $\begin{array}{l}\text { All calculated attributes } \\
\text { after Customizing Data to } \\
\text { the User Defined Extent } \\
\text { plus AREA }\end{array}$ & $\begin{array}{l}\text { Intermediate during Preparing for } \\
\text { Agent Analyst. The area is } \\
\text { important to calculate total seeds } \\
\text { and number of agents }\end{array}$ \\
\hline
\end{tabular}




\begin{tabular}{|l|l|l|}
\hline Name & Description & Importance \\
\hline $\begin{array}{l}\text { All_polygonvalues__ } \\
\text { forsubset2 }\end{array}$ & $\begin{array}{l}\text { All calculated attributes } \\
\text { after Preparing Data for } \\
\text { Agent Analyst (Section } \\
5.3\end{array}$ & $\begin{array}{l}\text { Used by Preparing for Agent } \\
\text { Analyst to calculate and place } \\
\text { random points that act as agents }\end{array}$ \\
\hline PrimaryRndPts_* & $\begin{array}{l}\text { Points representing } \\
\text { agents for the Primary } \\
\text { Movement Simulation }\end{array}$ & $\begin{array}{l}\text { Agent positions are edited by } \\
\text { Agent Analyst }\end{array}$ \\
\hline SecondaryPts_* & $\begin{array}{l}\text { Points representing } \\
\text { agents for the } \\
\text { Germination Simulation }\end{array}$ & $\begin{array}{l}\text { Agent positions are edited by } \\
\text { Agent Analyst }\end{array}$ \\
\hline Subset**_Postit***_Raw & Final output & $\begin{array}{l}\text { Final result that the user must } \\
\text { first classify before comparing it } \\
\text { to the original }\end{array}$ \\
\hline $\begin{array}{l}\text { Subset**_Postit***_- } \\
\text { NextIter }\end{array}$ & Classified final output & $\begin{array}{l}\text { Final result that may be } \\
\text { compared to the original dataset }\end{array}$ \\
\hline Subset**_Postit***_PA & $\begin{array}{l}\text { Presence/Absence } \\
\text { representation of the final } \\
\text { output }\end{array}$ & $\begin{array}{l}\text { Final result classified for } \\
\text { presence/absence comparisons }\end{array}$ \\
\hline
\end{tabular}

$*$ = trial number

** = subset number (if multiple subsets in one study area)

$* * *=$ iteration number

The next four datasets are the result of Attribute Calculations: HabitatProbabilities, SeedDensity, PlantDensity, and Outliers_Polygons. HabitatProbabilities contains values that are the basis for the Germination Simulation. SeedDensity contains values that are a few geoprocesses away from establishing the number of agents to partake in the Agentbased Model. PlantDensity contains values that influence the Primary Movement Simulation. And Outliers_Polygons defines areas to exclude.

The next two datasets are the result of Customizing Data for the Subset Area. All_polygonvalues_forsubset1 is used as input at a later point in the model but the SubsetPercentCover2001 simply represents the initial annual brome cover for the userdefined subset area. All_polygonvalues_forsubset1 contains all the values calculated by Attribute Calculations, which includes seed density, plant density, habitat probabilities, and wind.

The next three datasets are the result of Preparing Data for Agent Analyst: All_polygonvalues_wArea, All_polygonvalues_forsubset2, and PrimaryRndPts. All_polygonvalues_wArea is the result of adding the calculated area to the All_polygonvalues_forsubset1 dataset. All_polygonvalue_forsubset 2 uses the area and seed density to calculate a total number of seeds per polygon as well as the number of agents.

PrimaryRndPts and SecondaryPts are two datasets created by placing random points within polygon constraints and exported to the AA directory for the Primary Movement Simulation and Germination Simulation, respectively. Both datasets are linked to AA 
through the data source editor, allowing their model to inherit attributes. The implemented code then directly edits point positions depending on these attributes.

The last three datasets are the result of Post-Process Results: Subset**_Postit***_Raw, Subset**_Postit***_NextIter, and Subset**_Postit***_PA. Subset**_Postit***_Raw is the first version of the final model output. Subset**_Postit***_NextIter is classified on a comparable scale to the 2001 Annual Brome Cover dataset, is the dataset users can judge the most problematic areas as far as annual brome, and is the input dataset for another model iteration. Subset**_Postit***_PA is classified to represent annual brome presence and absence.

\subsection{0,000 Agents}

The final output of the model is symbolized by presence/absence as well as a gradient of colors representing percent cover (see Figure 9.1 and Figure 9.2); outlier areas are classified as a large absence and zero percent cover. No matter which representation is analyzed, the effect of fractional values caused by the generalization issue is quite evident. If the model was run only once, then lower amounts of annual brome have disappeared and higher amounts have spread significantly. After running the model two more times, there are little changes in spread, but there are fluctuations in the amount of cover. This represents systematic error - error within the model rather than caused by the user or original dataset. 


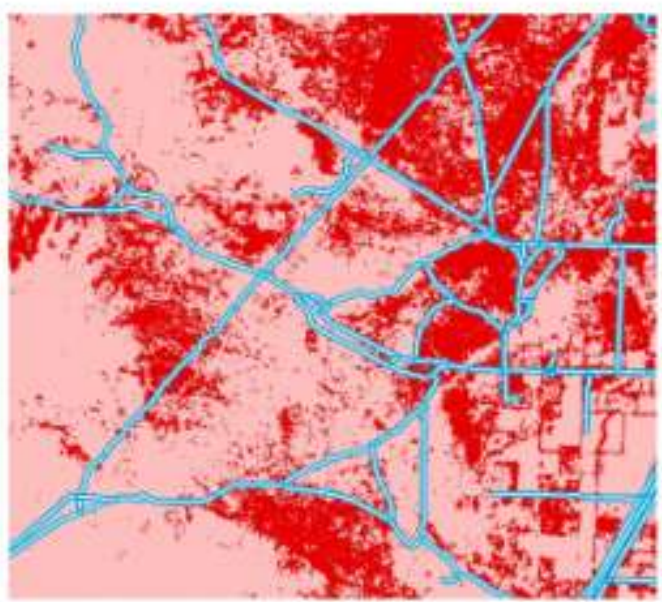

Annual Brome Cover (2001) -

Presence/Absence

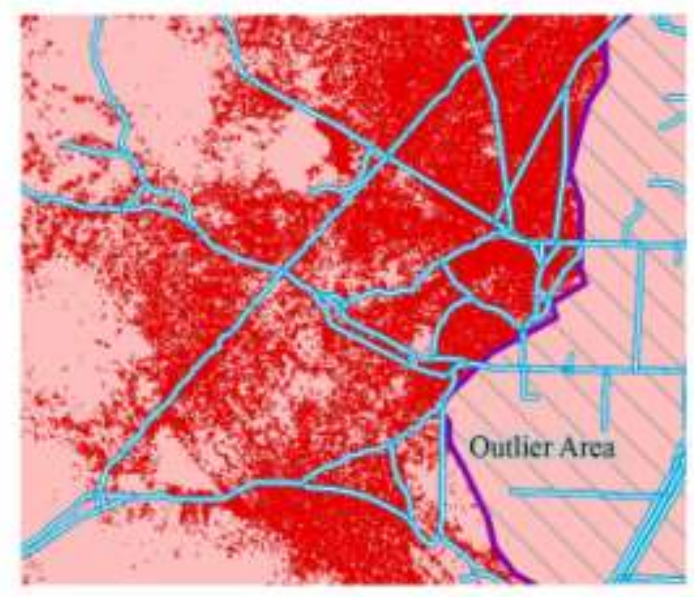

Annual Brome Cover (Post Iteration 41 ) Presence/Absence

Absent

Present

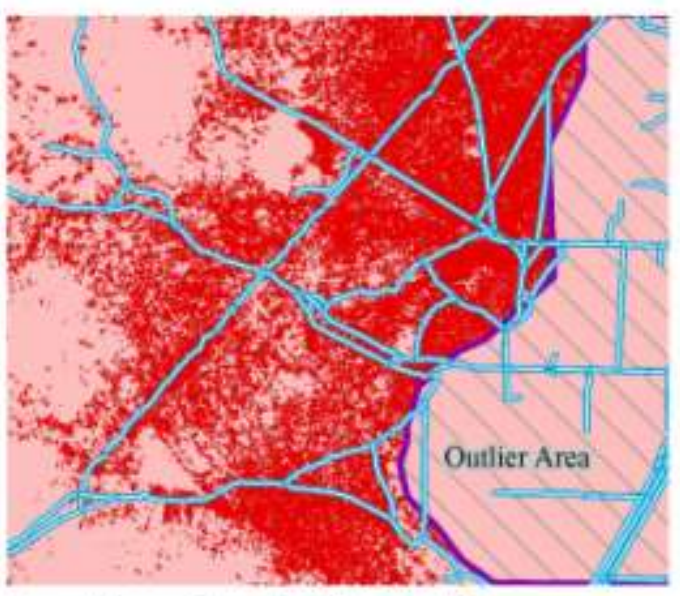

Annual Brome Cover (Post lteration \#2) Presence/Absence

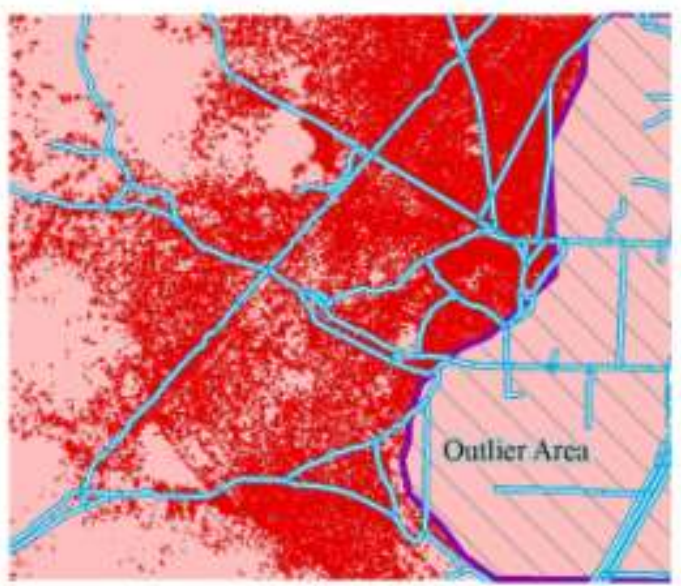

Annual Brome Cover (Post lteration \#3) -. Presence/Absence

Figure 9.1. Annual Brome Presence/Absence for 80 Thousand Agents 


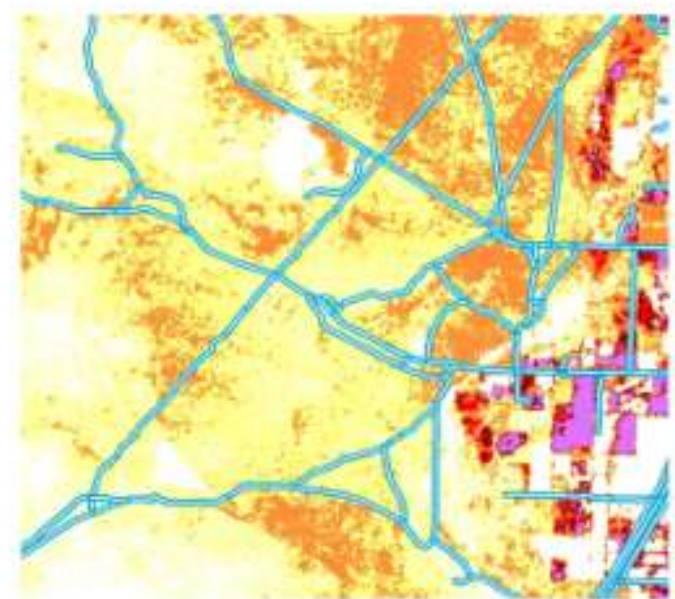

Annual Brome Cover (2001) Percent Cover

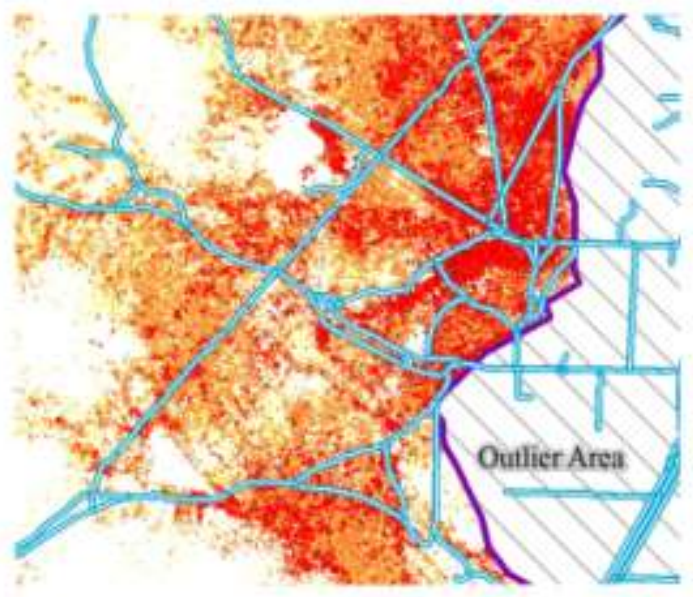

Annual Brome Cover (Post Iteration 41 ) Pereent Cover

VALUE
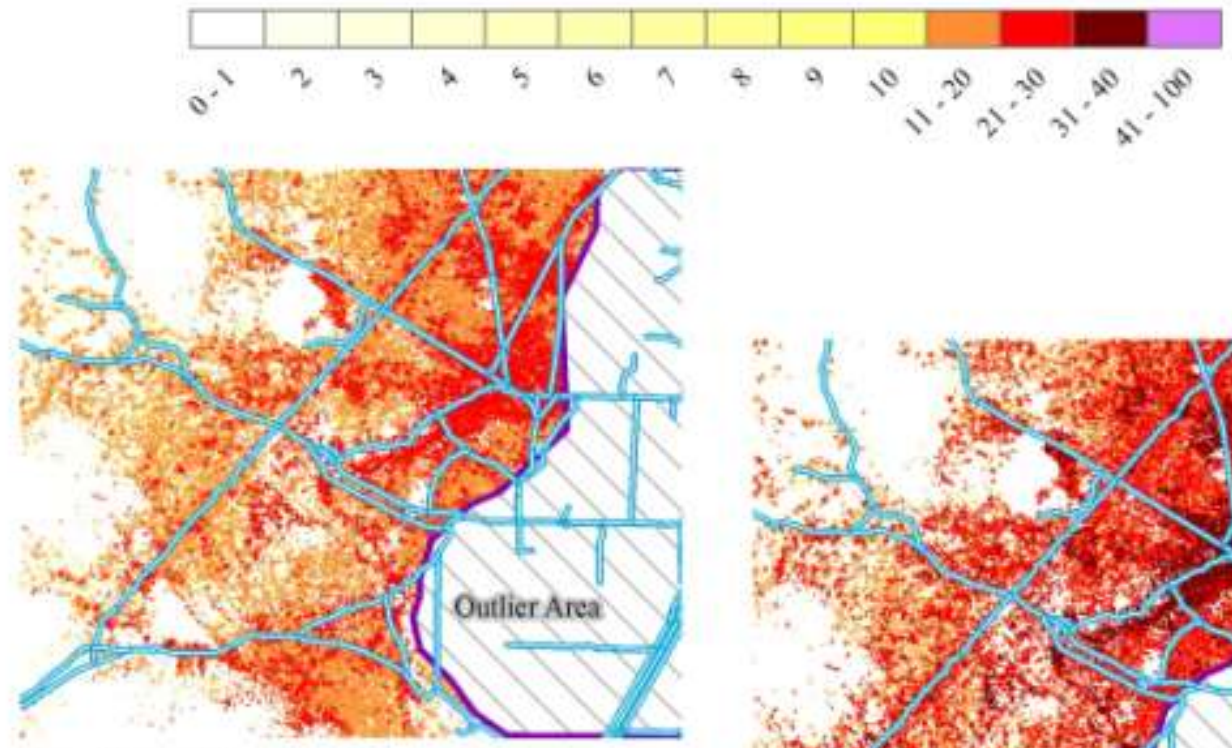

Annual Brome Cover (Post Iteration \#2) Percent Cover

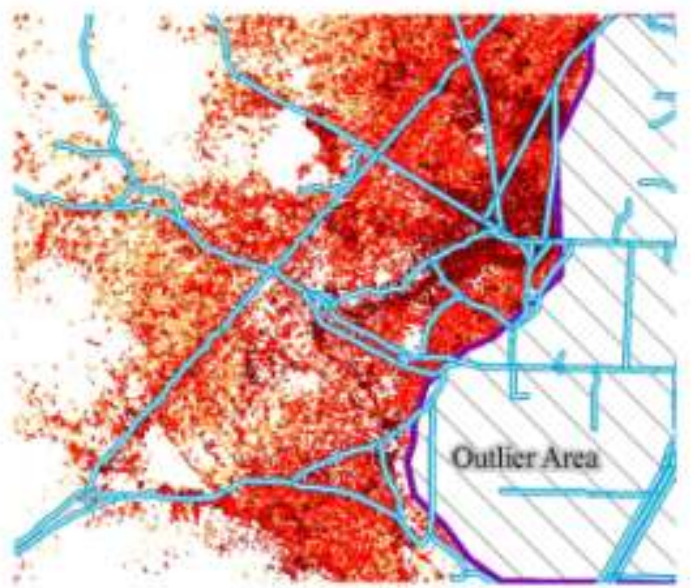

Annual Brome Cover (Post Iteration \#3) -. Percent Cover

Figure 9.2. Annual Brome Percent Cover for 80 Thousand Agents 
In the original dataset, an array of yellows symbolizes values between 1 and 10 . However, after a single run, some of these lower values are set to zero, causing erroneous results. If the solution described in Section 8.4.2 was applied, fractional values would be represented with higher accuracy in the final output.

When looking at the annual brome cover before and after the first model run, there is a significant increase in its range (see Table 9.3). In the original dataset, $19.98 \%$ of cells had presence and $80.02 \%$ of cells had absence, and after the first model run, $35.25 \%$ of cells had presence and $64.75 \%$ of cells had absence; an increase of roughly $15 \%$. The differences between the first, second, and third runs were not nearly as significant: roughly $2 \%$.

Table 9.3. Presence Statistics for 80 Thousand Agents

\begin{tabular}{|l|l|l|l|l|l|l|l|l|}
\hline Status & \multicolumn{2}{|l|}{ Zero (original) } & \multicolumn{2}{l|}{ One Iteration } & \multicolumn{2}{l|}{ Two Iterations } & \multicolumn{2}{l|}{ Three Iterations } \\
\hline Present & 45973 & $19.98 \%$ & 70855 & $35.25 \%$ & 68278 & $37.52 \%$ & 71153 & $39.20 \%$ \\
\hline
\end{tabular}

As described in Section 4.3.2, Peterson (2003) states that the 2001 Annual Brome Cover dataset does not represent absolute values but instead relative values; meaning they are comparable to one another but represent little individually. It appears that with each model run, the percent cover increases, but this would be based on observing the absolute values. Because fractional values are not included after a single model run, they do not have the same opportunity to increase in magnitude, causing a misrepresentation of relative comparisons.

There is also another unknown error in the model that is creating the increase in presence significantly after one iteration and then leveling off afterwards. Perhaps there is a relationship with the number of agents participating in the simulation and the number of cells with presence. Perhaps many more agents are necessary for a more accurate and realistic simulation. Either way, more testing would be needed to discover and mitigate the issue.

To further analyze the data that was produced, the percent cover values were plotted against the number of cells that represent them (see Figure 9.3). When zero percent cover is included in the distribution plot, the model results are significantly skewed because of both outlier exclusion and fractional values: a major portion of the analyzed area was urban, which was delineated and excluded from the agent-based model, resulting in many cells with a value of zero; many fractional values in the lower percent cover range were disregarded. 


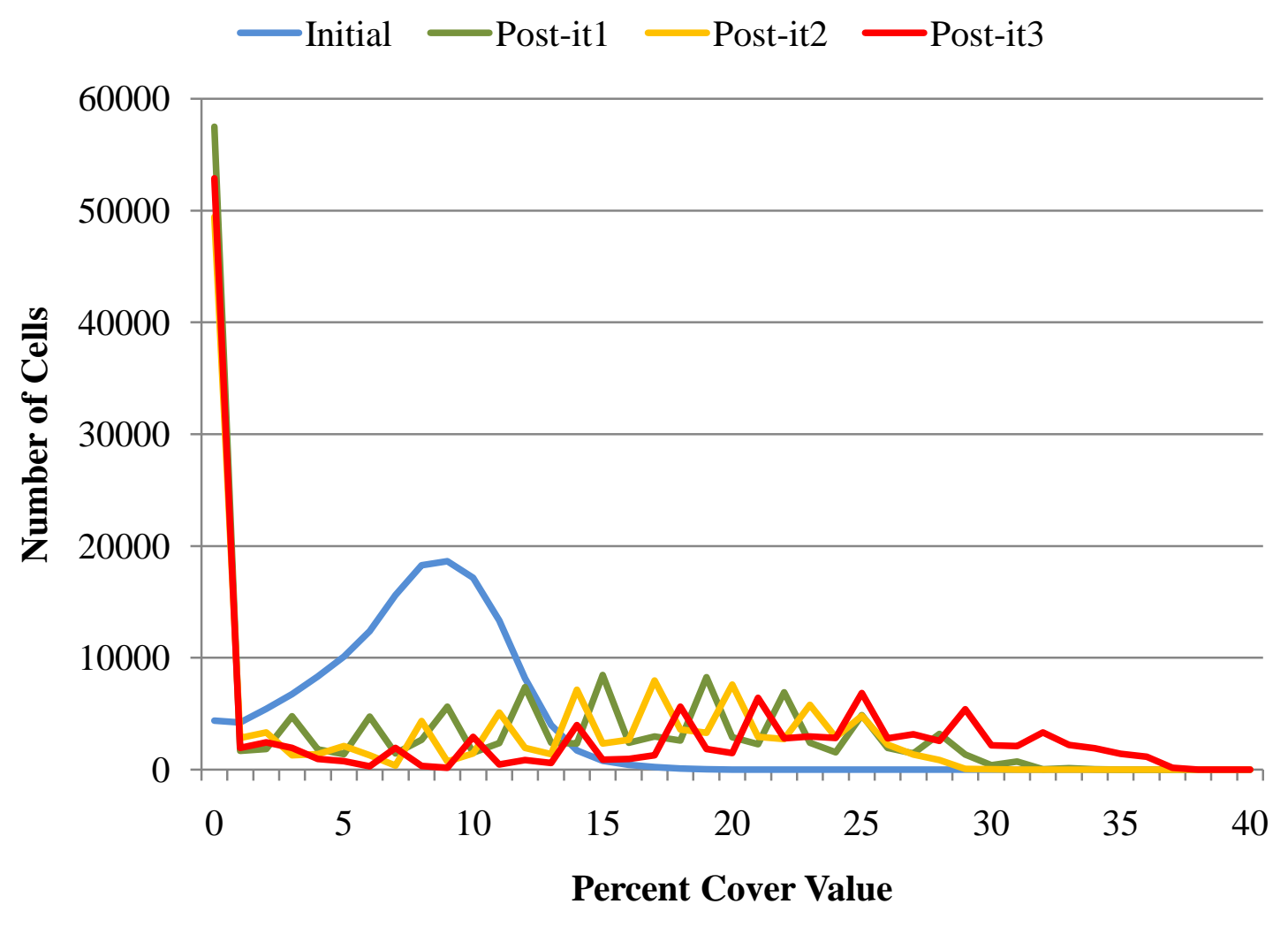

Figure 9.3. Percent Cover Values Plotted Against the Number of Cells Representing Them

When zero percent cover is excluded from the distribution plot, it is significantly less skewed and more interpretable (see Figure 9.4). The initial 2001 Annual Brome Cover dataset has a smooth distribution of values between 1 and 17, whereas the three classified datasets resulting from the agent-based model do not. The model results have many peaks and dips, creating a more chaotic distribution. However, there is a shift toward the higher percent cover values when taking into account the highest peak, the most frequent percent cover value: roughly 9 percent for the initial dataset; roughly 15 percent after one run; roughly 17 percent after two runs; and roughly 25 percent after three runs. 


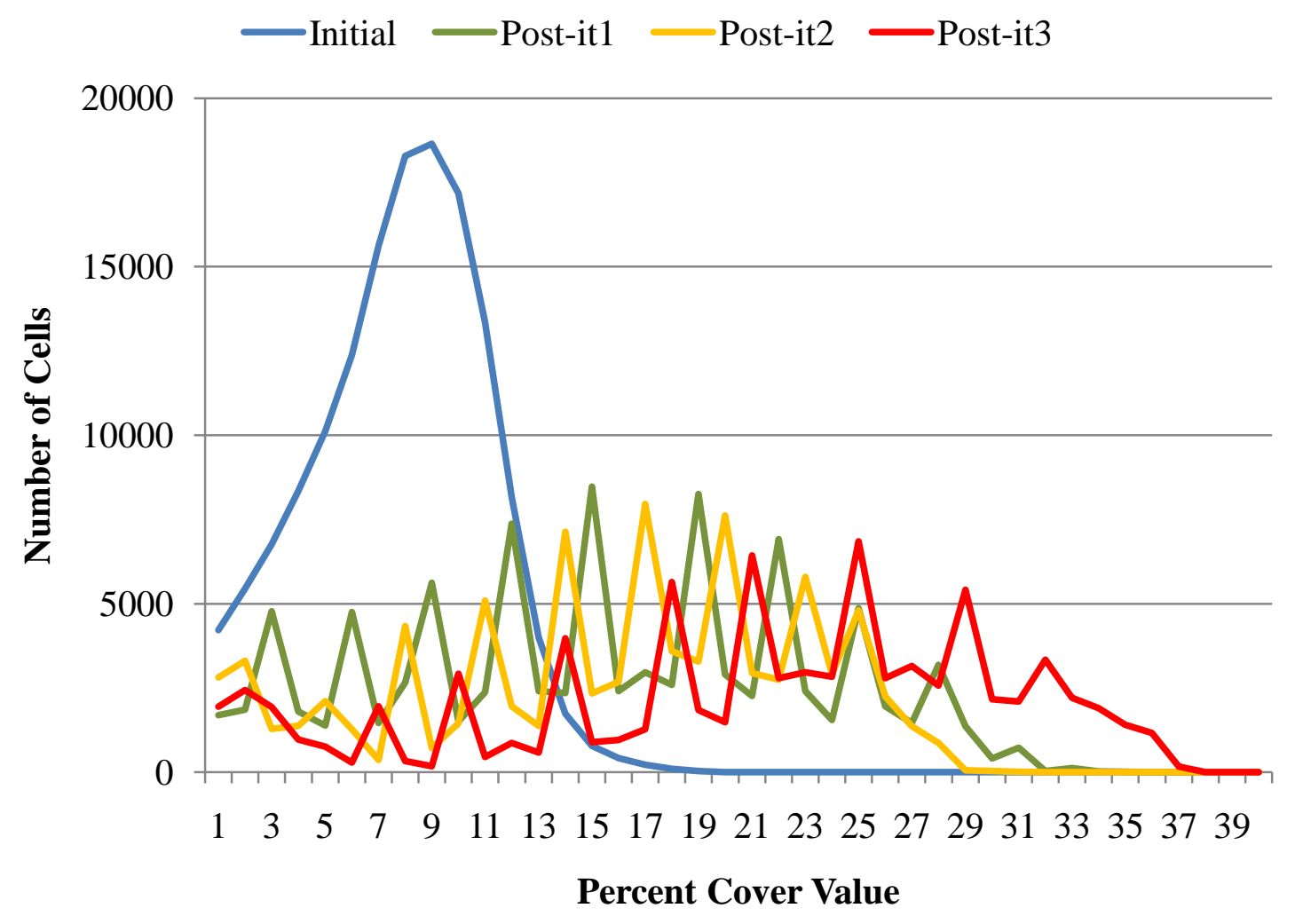

Figure 9.4. Percent Cover Values Plotted Against the Number of Cells Representing Them Excluding Zero

The periodicities in the model's results are most likely due to a form of systematic error that exists within the agent-based model. The exact systematic cause of these discrepancies is unknown, however they must be addressed in order for the results to be valid.

\subsection{0,000 Agents}

With fewer agents, the effect of ignoring values is more prevalent (see Figure 9.5 and Figure 9.6). The annual brome range appears to significantly decrease after the first model run because of systematic error. The next two runs do not have the same magnitude of change but rather make the existing percent cover values increase significantly. 


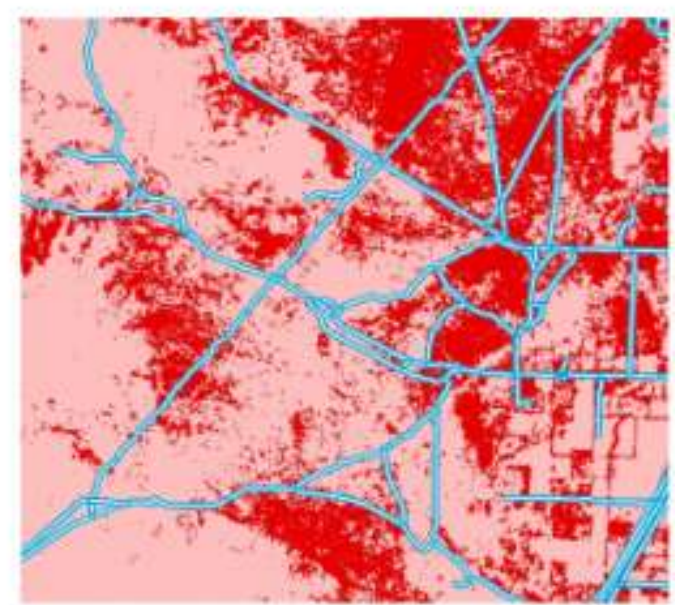

Annual Brome Cover (2001) Presence/Absence

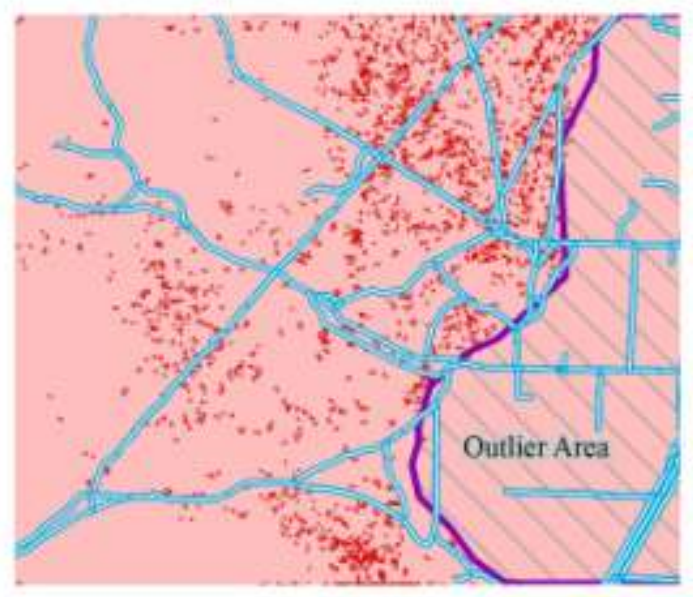

Annual Brome Cover (Post Iteration $\#$ 1) Presence/Absence

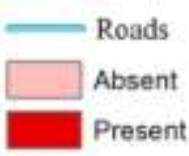

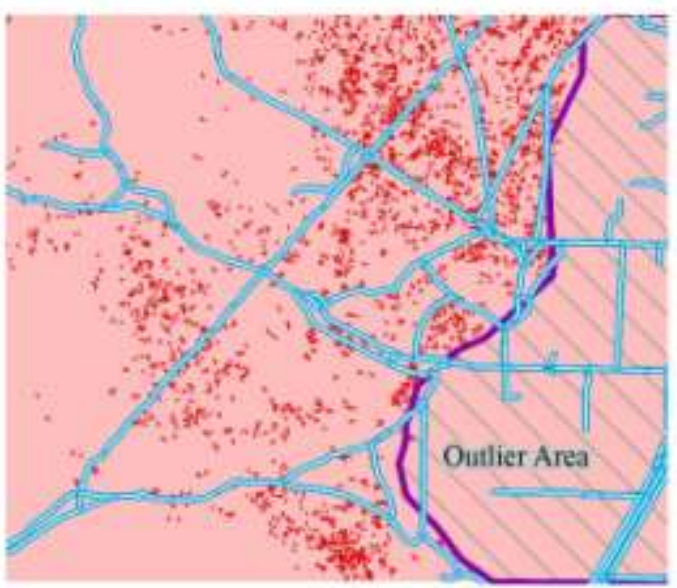

Annual Brome Cover (Post lteration $\$ 2$ ) Presence/Absence

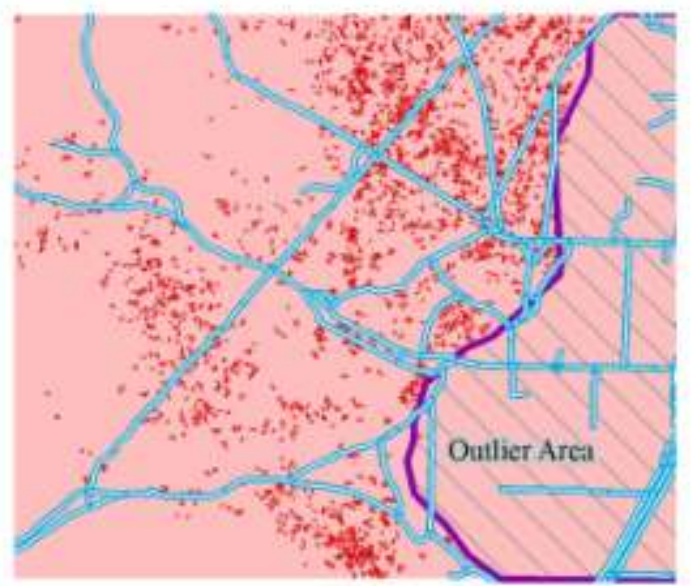

Annual Brome Cover (Post Iteration \#3) Presence/Absence

Figure 9.5. Annual Brome Presence/Absence for 10 Thousand Agents 


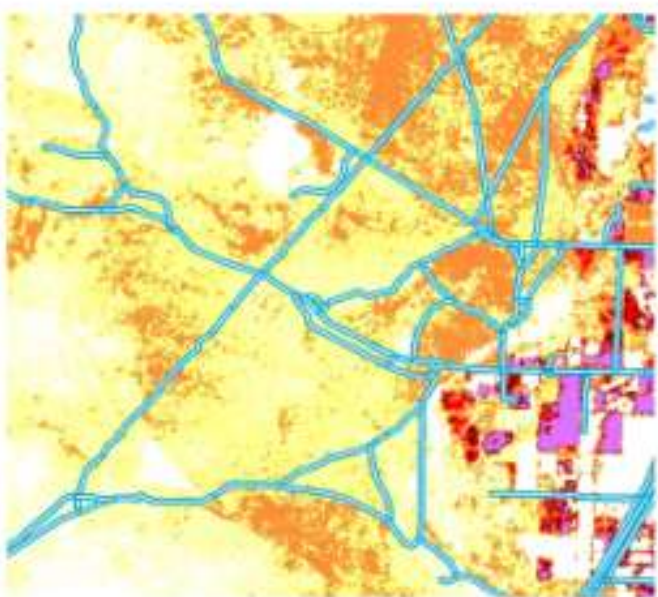

Annual Brome Cover (2001) Percent Cover

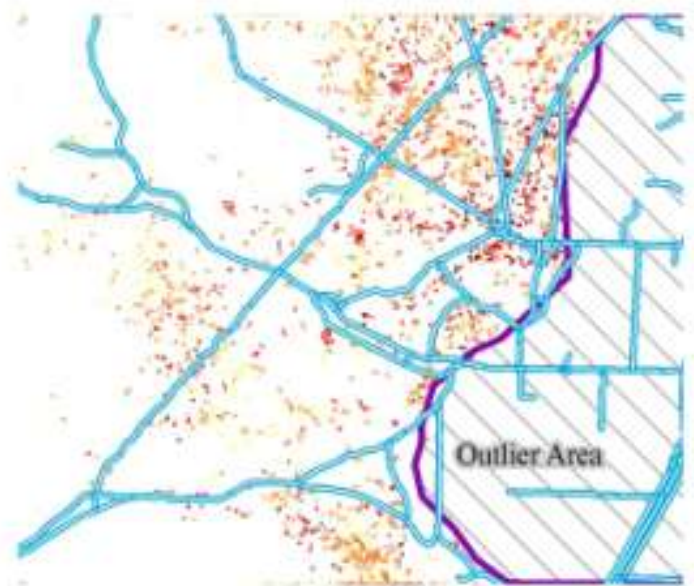

Anmual Brome Cover (Post Iteration स1) Pereent Cover

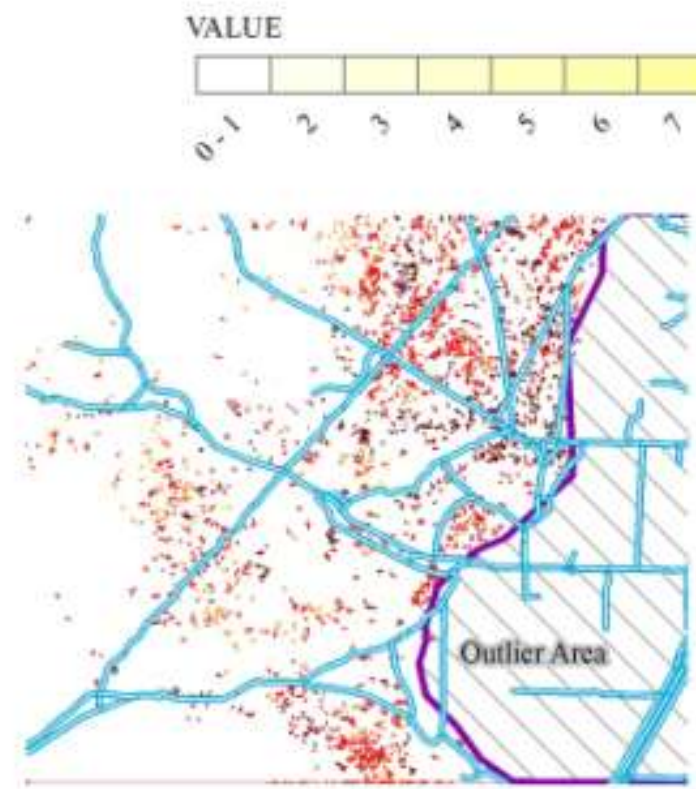

Annual Brome Cover (Post lieration \#2) Percent Cover

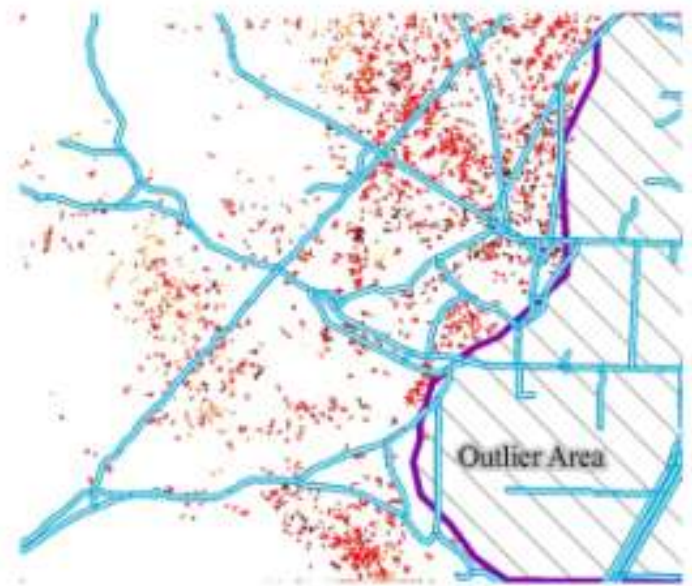

Annual Brome Cover (Post Iteration \#3) Percent Cover

Figure 9.6. Annual Brome Percent Cover for 10 Thousand Agents 
When looking at the annual brome cover before and after the first model run, it seems there is a significant decrease in its range (see Table 9.4). In the original dataset, $20.91 \%$ of cells had presence and $79.09 \%$ of cells had absence, and after the first model run, $5.49 \%$ of cells had presence and $94.51 \%$ of cells had absence; a decrease of roughly $15 \%$. The differences between the first, second, and third runs were not nearly as significant: less than $1 \%$.

Table 9.4. Presence Statistics for 10 Thousand Agents

\begin{tabular}{|l|l|l|l|l|l|l|l|l|}
\hline Status & \multicolumn{2}{|l|}{ Zero (original) } & \multicolumn{2}{l|}{ One Iteration } & \multicolumn{2}{l|}{ Two Iterations } & \multicolumn{2}{l|}{ Three Iterations } \\
\hline Present & 43096 & $20.91 \%$ & 9387 & $5.49 \%$ & 10835 & $6.01 \%$ & 11125 & $6.17 \%$ \\
\hline
\end{tabular}

The fractional value problem is worse with fewer agents. With fewer agents to distribute, 10 thousand as opposed to 80 thousand, only the highest percent values are significant enough to receive an agent. This generates more skew, reducing model accuracy.

\subsection{Early Development: 250,000 Agent Maximum}

During the original testing of the annual brome agent-based model, it was thought that the prototype area could be broken down into $2110.5 \times 14$ kilometer partitions, and the one with the largest amount of high percent cover values would receive a 250,000 agent maximum (see Figure 9.7 and Figure 9.8). This, in turn, determined a division factor (this process was described in Section 8.4). Each partition was then divided by that value creating $\mathrm{X}$ number of agents. 


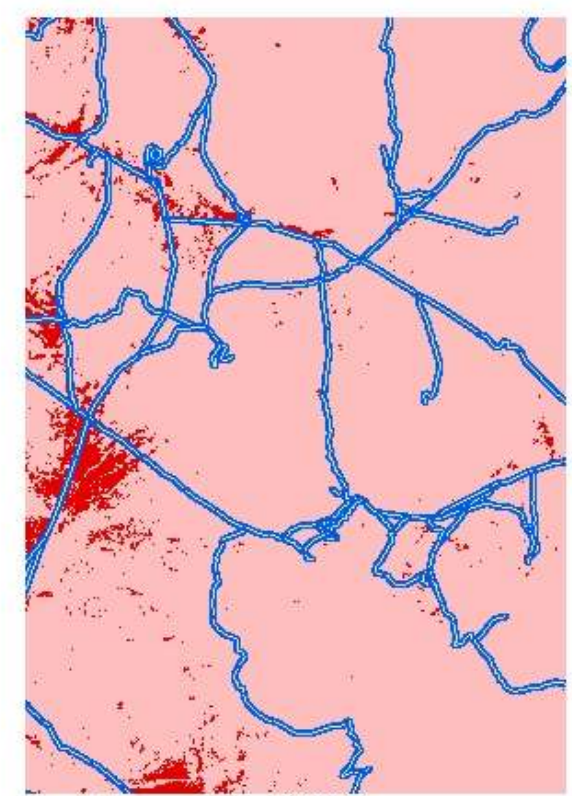

Annual Brome Cover (2001) --

Presence/Absence

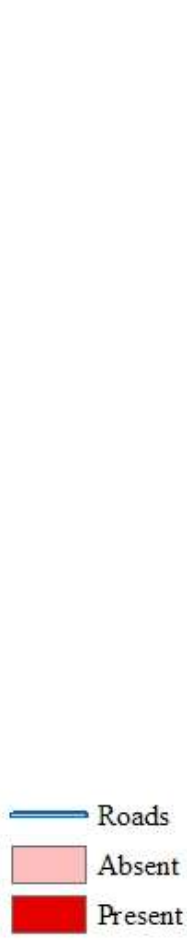

Annual Brome Cover (Post Iteration \#2) -Presence/Absence

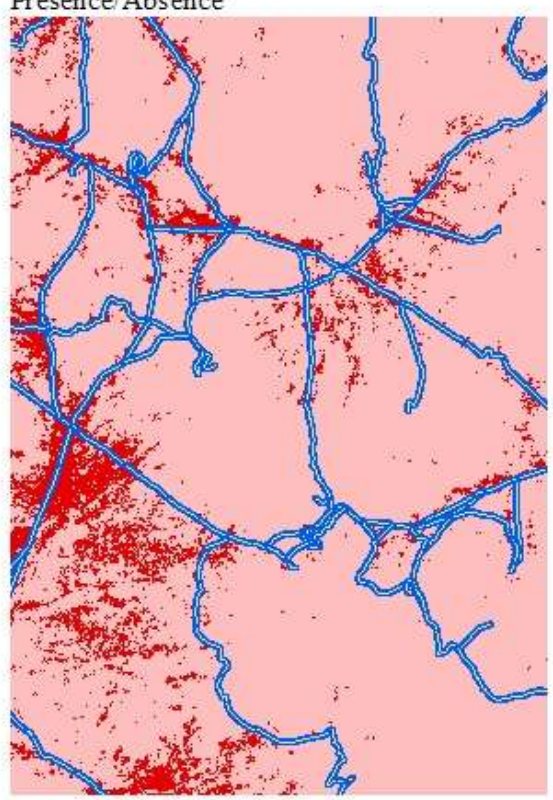

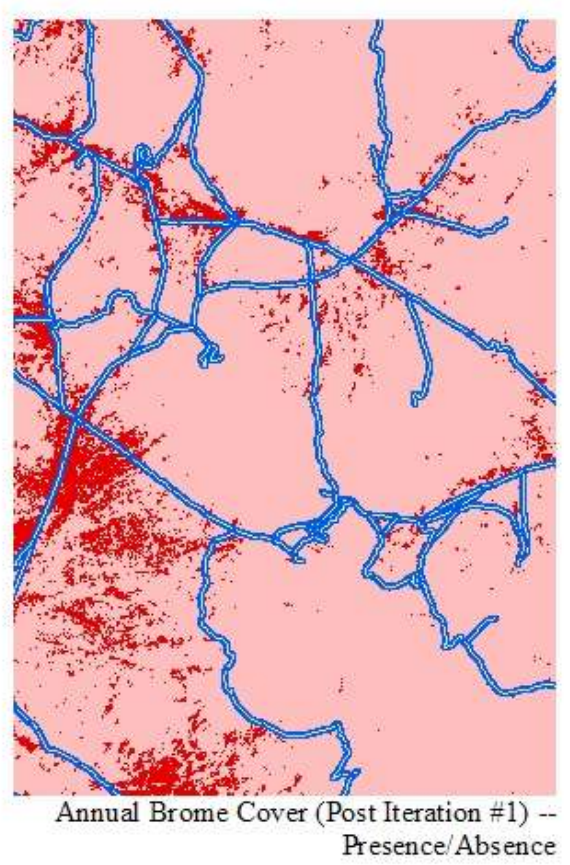

Presence/Absence

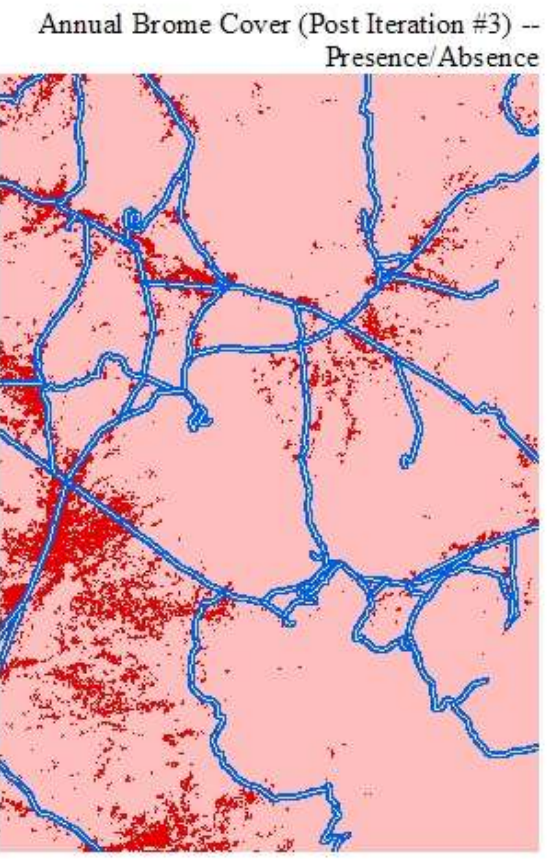

Figure 9.7. Annual Brome Presence/Absence for 250 Thousand Agents 


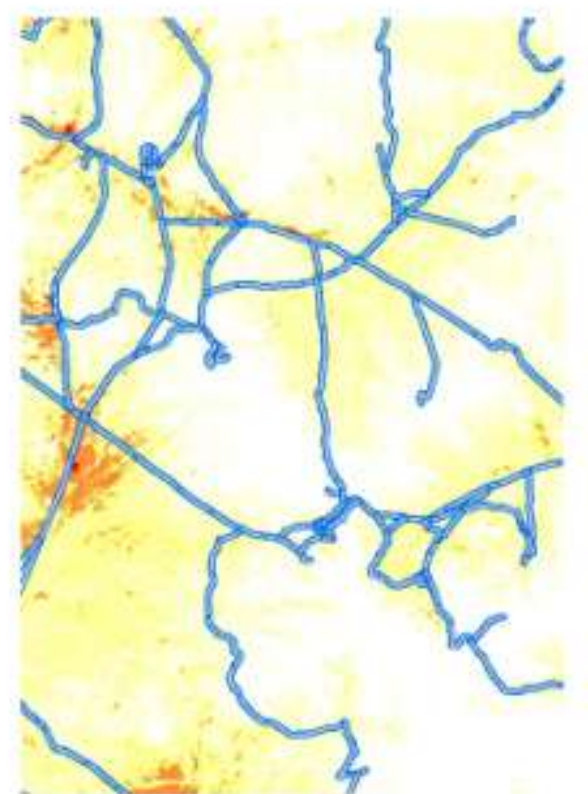

Annual Brome Cover (2001) Percent Cover

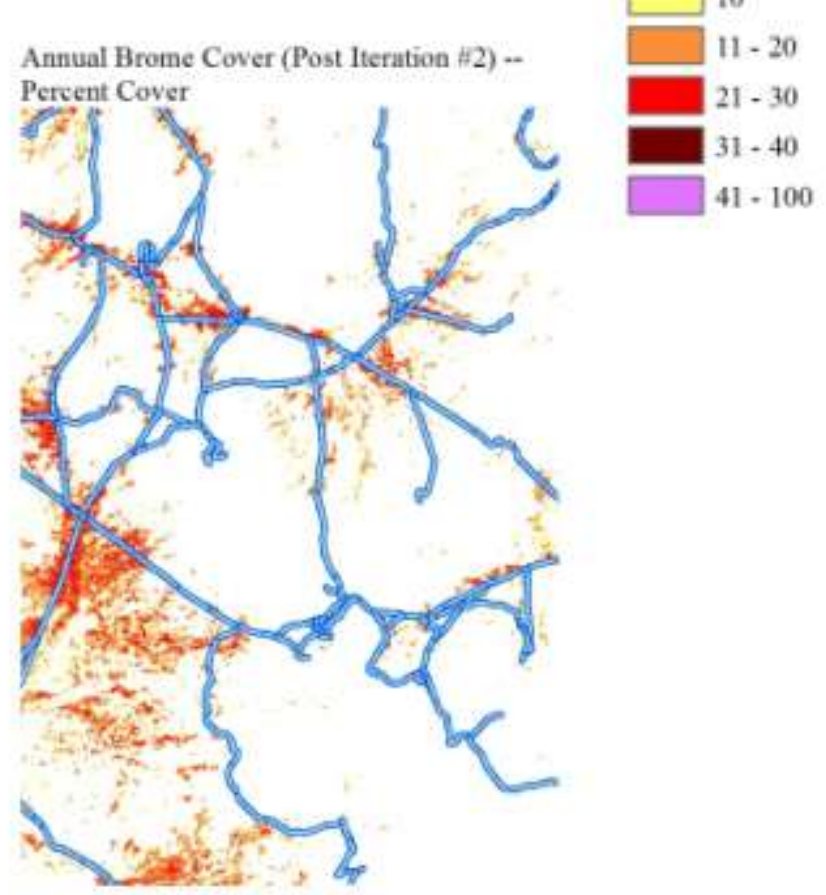

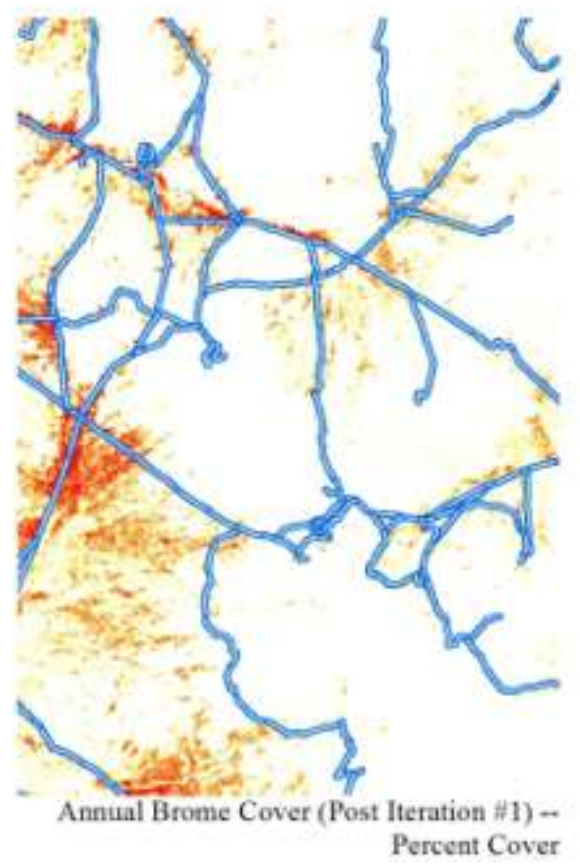

Annual Brome Cover (Post Iteration H3) -

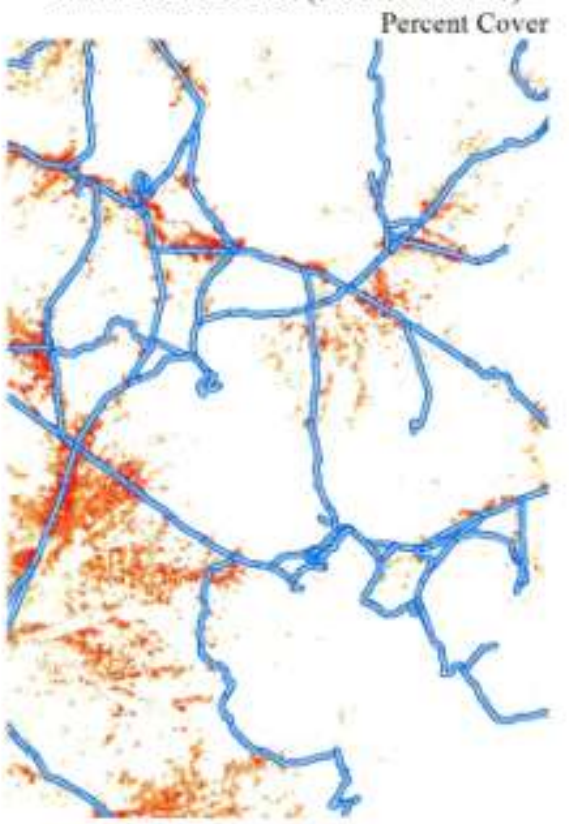

Figure 9.8. Annual Brome Percent Cover for 250 Thousand Agents 
When looking at the annual brome cover before and after the first model run, there is again an increase in its range (see Table 9.5). In the original dataset, $26.00 \%$ of cells had presence and $74.00 \%$ of cells had absence, and after the first model run, $28.03 \%$ of cells had presence and $72.00 \%$ of cells had absence; an increase of roughly $2 \%$. The differences between the first, second, and third runs were negative by roughly $1 \%$.

Table 9.5. Presence Statistics for 250 Thousand Agents

\begin{tabular}{|l|l|l|l|l|l|l|l|l|}
\hline Status & \multicolumn{4}{|l|}{ Zero (original) } & One & \multicolumn{2}{l|}{ Two } & \multicolumn{2}{l|}{ Three } \\
\hline Present & 1044550 & $26.00 \%$ & 1070442 & $28.03 \%$ & 1029041 & $27.00 \%$ & 899579 & $23.57 \%$ \\
\hline
\end{tabular}




\section{Conclusions}

As the first step toward integrating an agent-based model into a web-enabled decision support system for annual brome, this project explored capabilities and limitations. Although there was systematic error, testing the capabilities of ArcGIS software to partake in a dynamic simulation proved to be a significant contribution to modelers of annual brome spread and the agent-based modeling community.

The results are to be analyzed on a relative basis since the initial dataset indicates percent cover in relation to the other values. High final percent cover values represent an area with the largest probability of having annual brome. Lower percent cover values represent an area with the smallest probability of having annual brome. These probabilities would improve in accuracy with further adaptations discussed in Chapter 11.

As of now, the applied model rules indicated an overall migration of seeds in certain patterns. The longest spread distance occurred via roads, whereas a progressive alldirectional spreading was caused by wind.

Additionally, there were some discrepancies not explored by this project but are worth noting:

1) Is this final data representing the real world or what the model was told to do? When models are created, where specific rules dictate specific actions, other influential elements may be ignored. But does this have a significant impact? This model should be taken as an indicator of primary and secondary movement and not as a simulation of total annual brome dispersal.

2) By basing a model on a dataset with a cell size (30 meters) that is larger than some of the primary movements (less than 15 meters), any error propagation and inaccuracies caused by the data manipulation may appear as agent movements. Generalizing this model to a larger spatial scale, not accounting for individual seed movements but rather a flow, could increase the accuracy at the ecosystem level, but this may be too broad for the intended purpose of land management; managers need data for a specific AOI at a larger map scale.

3) Inaccuracies in the data's inter-format conversions could also be mistaken for agent movement. When rasters are converted into polygons, sometimes a raster cell and the polygon representing it are misaligned. It was concluded that when this occurs, the conversion is somehow using the raster cell centroid as a guide in producing the polygon boundaries. If the raster cell is 30 meters by 30 meters, this shift could result in a 15 meter skew after every conversion. This number could cause significant error propagation, especially in this model where the primary movement is typically 15 meters or less.

4) After the union of the wind, seed density, plant density, and habitat probabilities layers, several slivers, no larger than five meters in width, were sporadically produced within the new dataset. As a precaution, the union tolerance was increased to five meters to eliminate these slivers. By doing so, however, this may have created polygons that are representing larger or smaller areas than were initially established by the original raster dataset. 



\section{Future Work}

\subsection{Is it Possible Using Only ArcGIS Desktop?}

As the project progressed, it was realized that ArcToolbox has all the capability to accomplish all the simulation's processes run in AA. Although the model might involve hundreds more geoprocesses, it could be done. This would open up the possibility of using a geodatabase. A proper geodatabase design would make geoprocessing more efficient, and by not having to keep most data in the shapefile format, this allows data to be store in datasets, feature classes, and raster catalogs. This would boost computer efficiency and reduce model run times, as well as relax the 80,000 agent limit.

With the release of ArcGIS 9.3 in late June, it is expected that there will be further improvements that could significantly boost model efficiency: the ability to run python scripts in process, which would make it easier to incorporate complex scripts into a geoprocessing string; the ability to run a model as a loop, allowing a user to define the number of iterations to implement, initiating the model only one time.

The most common geoprocessing tools used in this model are the combinations of Add Field and Calculate Field. These tools establish and edit new fields, which are essential to incorporate equations and alterations in agent positions. Additionally, instead of directly editing a file's attributes, like in AA, ArcMap can create new feature classes based on updated fields. If a model was to incorporate a geodatabase, the model output and their field maps could be predefined.

\subsection{Real-Time Running Model}

It is difficult to incorporate a model that uses software other than ArcGIS into an ArcGIS Server web application. The derived data may be posted in the map, but ArcGIS's live geoprocessing capability cannot be utilized. An all ArcGIS agent-based model, however, could allow for such a feat, if engineered while keeping computer efficiency in mind. No doubt a model containing all the geoprocesses necessary would be nothing less than massive, but with the rapid advances in server and desktop technologies, it may be possible in the near future.

One component that Armstrong's project lacked, mentioned in his future directions chapter, was the ability to conduct live geoprocessing on web portal data. For land managers, it would be beneficial to overlay supplementary data, such as fire regimes, vegetation flammability, etc and utilize these layers in geoprocessing tasks. This would eliminate the need for a server geodatabase and would only require the server to conduct the analysis and store the results in a temporary file. Although this project did not explore the capabilities of ArcGIS Server 9.3, it is believed that it will contain the tools necessary to create a web application that accomplishes Armstrong's vision.

\subsection{Visualization}

Creating static datasets from other static datasets show specific times in the past and future ignoring the intermediates. This makes visualizing the process in which a dataset gained certain values difficult. With advances in cinematography, terrain representation, 
4-D visualization, and agent-based modeling, it is only a matter of time before more accurate static datasets and real-time movies for the dynamic processes can be created. There is significant educational value in modeling an ecological process through 4-D representation; and to make that simulation a case study of a specific location would be beneficial to land managers, homeowners, and insurers.

\subsection{Other Work}

Because of the numerous datasets produced with this model, organization is essential. Unfortunately with each run of the model, changing the output names within the model could take hours and overwriting previous outputs permanently deletes the initial dataset. Researching and implementing a way to have the model automatically tag a changing variable, such as data and time or iteration number, would open another level of organization and efficiency.

In the 2001 Annual Brome Cover dataset, the metadata described ten percent as the cutoff between presence and absence. Because the natural environment is never black and white but rather has fuzzy transitions, the seed density calculation had a separate equation for values between one and ten. Was this equation accurate in its representation? Should the curve be more or less steep in its exponential magnitude? It is believed the answer to these questions involves further investigating the field verification method used during the original dataset creation as well as visiting the prototype area in present day.

Lastly, a new primary movement equation is needed. The equation used, based on a small sample size, is outdated and has a poor correlation coefficient. By simply applying a more accurate study or conducting a study on seed movement caused by wind, this model's accuracy could be further improved. 


\section{References}

Armstrong, C. E. (2007). Annual Brome (Bromus tectorum) Wildfire Fuel Breaks: Webenabled GIS Wildfire Model Decision Support System. Unpublished Major Individual Project, University of Redlands, Redlands, CA.

BASF (Writer) (2006). Cheatgrass: A Burning Issue [Documentary]. Triangle Park, NC: BASF Corporation: Professional Vegetation Management.

BASF. (2008). BASF Group: Products by Segments. Retrieved 22 Feb, 2008, from http://www.corporate.basf.com/en/produkte/segmente.htm?id=41hUvC9t4bcp1s $\underline{\mathrm{W}}$

Beckler, A. A., French, B. W., \& Chandler, L. D. (2005). Using GIS in Areawide Pest Management: A Case Study in South Dakota. Transactions in GIS, 9(2), 109.

Bitterlich, W., Alsawydami, S., \& Douglas, D. (1993). Raster Modeling in GIS. Proceedings of the Canadian Conference on GIS, 1002-1011.

Booth, M. S., Caldwell, M. M., \& Stark, J. M. (2003). Overlapping resource use in three Great Basin species: Implications for community invasibility and vegetation dynamics. Journal of Ecology, 91(1), 36-48.

Bradley, B., \& Mustard, J. (2006). Characterizing the Landscape Dynamics of an Invasive Plant and Risk of Invasion Using Remote Sensing. Ecological Applications, 16(3), 1132-1147.

Esh, H. (2006). The Wildland/Urban Interface: Cheatgrass, Fire Danger, and, Fuel Breaks. Unpublished Major Individual Project, University of Redlands, Redlands, CA.

ESRI. (2006). Census TIGER/Line Data. Retrieved 20 May, 2007, from http://www.esri.com/data/download/census2000_tigerline/index.html

Frederick, K. (2007). 2007 Fire Season in Review - an Era of Change. Natioanal Intragency Fire Center: Science \& Stewardship.

Gardner, M. (1970). Mathematical Games: The fantastic combinations of John Conway's new solitaire game "life". Scientific American (223), 120-123.

Giles, T. (2007). CalcFieldSum.py. Retrieved 06 June 2008, from http://support.esri.com

Gillham, J. H. (2001). Invasive Species Inventory and Weed Susceptibility Prediction Model for Southwestern Wyoming. University of Wyoming, Laramie.

Gimblett, H. R. (2002). Integrating Geographic Information Systems and Agent-Based Technologies for Modeling and Simulating Social and Ecological Phenomena. In H. R. Gimblett (Ed.), Integrating Geographic Information Systems and Agentbased Modeling Techniques (pp. 1-20). New York, New York: Oxford University Press.

Heuvelink, G. B. M. (2000). Error Propagation in Environmental Modelling. New York, NY: Taylor \& Francis. 
Hulbert, L. C. (1955). Ecological Studies of Bromus tectorum and Other Annual Bromegrasses. Ecological Monographs, 25(2), 181-213.

Interior Columbia Basin Ecosystem Management Project. (1995). GIS Data. Retrieved 05 April, 2008, from http://www.icbemp.gov/

Klug, M. (2007). 2007 Fire Management Report: Nevada Division of Forestry - Western Division.

Laffra, C. (N.D.). Dijkstra's Shortest Path Algorithm. Retrieved 23 April, 2008, from http://www.nist.gov/dads/HTML/dijkstraalgo.html

Longley, P. A., Goodchild, M. F., Maguire, D. J., \& Rhind, D. W. (2005). Geographical Information Systems and Science (2nd ed.). England: John Wiley \& Sons.

Minar, N., Burkhart, R., Langton, C., \& Askenazi, M. (1996). The Swarm Simulation System: A Toolkit for Building Multi-agent Simulations. Working Paper 96-06042, Santa Fe Institute, Santa Fe.

MIT Teacher Education Program. (2008). Starlogo TNG: Welcome to the Next Generation. Retrieved 28 March, 2008, from http://education.mit.edu/starlogotng/

Mulligan, M., \& Wainwright, J. (Eds.). (2004). Modelling and Model Building. England: John Wiley \& Sons.

Neese, E. (2000). Cheatgrass Bromus tectorum L. Retrieved 13 October 2007, from http://plants.usda.gov/plantguide/pdf/pg_brte.pdf

North, M. J., \& Macal, C. M. (2007). Managing Business Complexity: Discovering Strategic Solutions with Agent-Based Modeling and Simulation. New York, NY: Oxford University Press.

Novak, S. J., \& Mack, R. N. (2001). Tracing Plant Introduction and Spread: Genetic Evidence from Bromus tectorum (Cheatgrass). . BioScience, 51(2), 114-122.

Perry, G. L. W., \& Bond, N. R. (Eds.). (2004). Spatial Population Models for Animals. England: John Wiley \& Sons.

Peters, D. (2007). An ESRI Technical Reference Document: System Design Strategies. Retrieved March 14, 2008, from http://www.esri.com/library/whitepapers/pdfs/sysdesig.pdf

Peterson, E. (2003). Mapping Percent-Cover of the Invasive Species Bromus Tectorum (Cheatgrass) Over a Large Portion of Nevada from Satellite Imagery. Carson City, Nevada: Nevada Natural Heritage Program.

Price, M. (2005). Modeling Topography and Fuels at the Wildland/Urban Interface. ArcUser, Jan. - Mar. 2005, 58-63.

Repast Home Page. (2008). Repast: Recursive Porous Agent Simulation Toolkit. Retrieved 11 March, 2008, from http://repast.sourceforge.net/repast_3/index.html

Sagebrush Bird Conservation Network. (N.D.). SBCN Photo Gallery. Retrieved 13 July 2008, from http://www.sagebrushbird.org/gallery.html 
The Wilderness Society. (2006). Facts: 2006 Fire Season To-Date by the Numbers, 21 March 2008, from

http://www.wilderness.org/Library/Documents/upload/WildfireSeason2006_Octo ber31.pdf

Tobler, W. R. (1979). Cellular Geography. In S. Gale \& G. Olsson (Eds.), Philosophy in Geography (pp. 379-386). Dordrecht, Holland: D. Reidel Publishing Company.

Updadhyaya, M. K., Turkington, R., \& McIlvride, D. (1986). The Biology of Canadian Weeds: 75. Bromus tectorum L. Canadian Journal of Plant Science, 66, 689-709.

USGS. (1999). Seamless Data Distribution System. Retrieved 04 December, 2007

von Neumann, J. (Ed.). (1963). The General and Logical Theory of Automata (Vol. 5).

von Neumann, J. (Ed.). (1966). Theory of Self-Reproducing Automata.

Wolfram, S. (1983). Statistical Mechanics of Cellular Automata. In S. Wolfram (Ed.), Cellular Automata and Complexity (pp. 1-69). Menlo Park, CA: Addison-Wesley Publishing Company.

Young, J. A. (1978). Population Dynamics After Wildfires in Sagebrush Grasslands. Journal of Range Management, 31(4), 283-289.

Young, J. A. (2000). Bromus tectorum L. In C. C. Bossard, J. M. Randall \& M. C. Hoshovsky (Eds.), Invasive Plants of California's Wildlands (pp. 76-80). Berkeley, CA: University California Press.

Zouhar, K. (2003). Bromus tectorum. In: Fire Effects Information System, [Online]. Retrieved 16 October, 2007, from http://www.fs.fed.us/database/feis/ 



\section{Appendix A - Agent-based Modeling Supplement Materials}

Agent Analyst Supplement Materials -

$<$ http://www.institute.redlands.edu/agentanalyst/AgentAnalyst.html> (Redlands Institute, 2008).

Repast Supplement Materials -

$<$ http://repast.sourceforge.net/repast_3/index.html> (Repast Home Page, 2008).

Starlogo TNG Supplement Materials -

<education.mit.edu/starlogo-tng/> (MIT Teacher Education Program, 2008).

Swarm Supplement Materials -

<www.swarm.org> (Center for the Study of Complex Systems, 2008). 



\section{Appendix B - Definitions}

Agent overload: a model run-time failure due to limitations in computer hardware or the excessive number of wanted agents.

Buffer \#1: the buffer in which to identify road agents; the buffer is 3.1336 meters on each side of the line feature (including the one meter shoulder).

Buffer \#2: the 2-one meter buffer sections that represent the road shoulders.

Prototype Area: a rectangular $33.5 \mathrm{~km}$ by $98 \mathrm{~km}$ section of northwest Nevada; the total extent in which there sufficient data to run the agent-based model.

Study Area: the entire user's area of interest to analyze using the agent-based model.

Subset Area/Subsection: a smaller section of the study area extracted to fit the computer's capabilities and user's time constraints.

Repolygonization: the process of converting a data format back into vector polygons.

Tick: the Agent Analyst simulation time unit.

Value Set \#1: percent cover values ranging from 10 to 40.

Value Set \#2: percent cover values ranging from 1 to 10.

Value Set \#3: percent cover values equaling 0 or more than 40.

Value Set \#4: percent cover values ranging from 10 to 40.

Value Set \#5: percent cover values equaling less than 10.

Value Set \#6: percent cover values equaling more than 40. 



\section{Appendix C - Seeds per Meter Calculation Model}

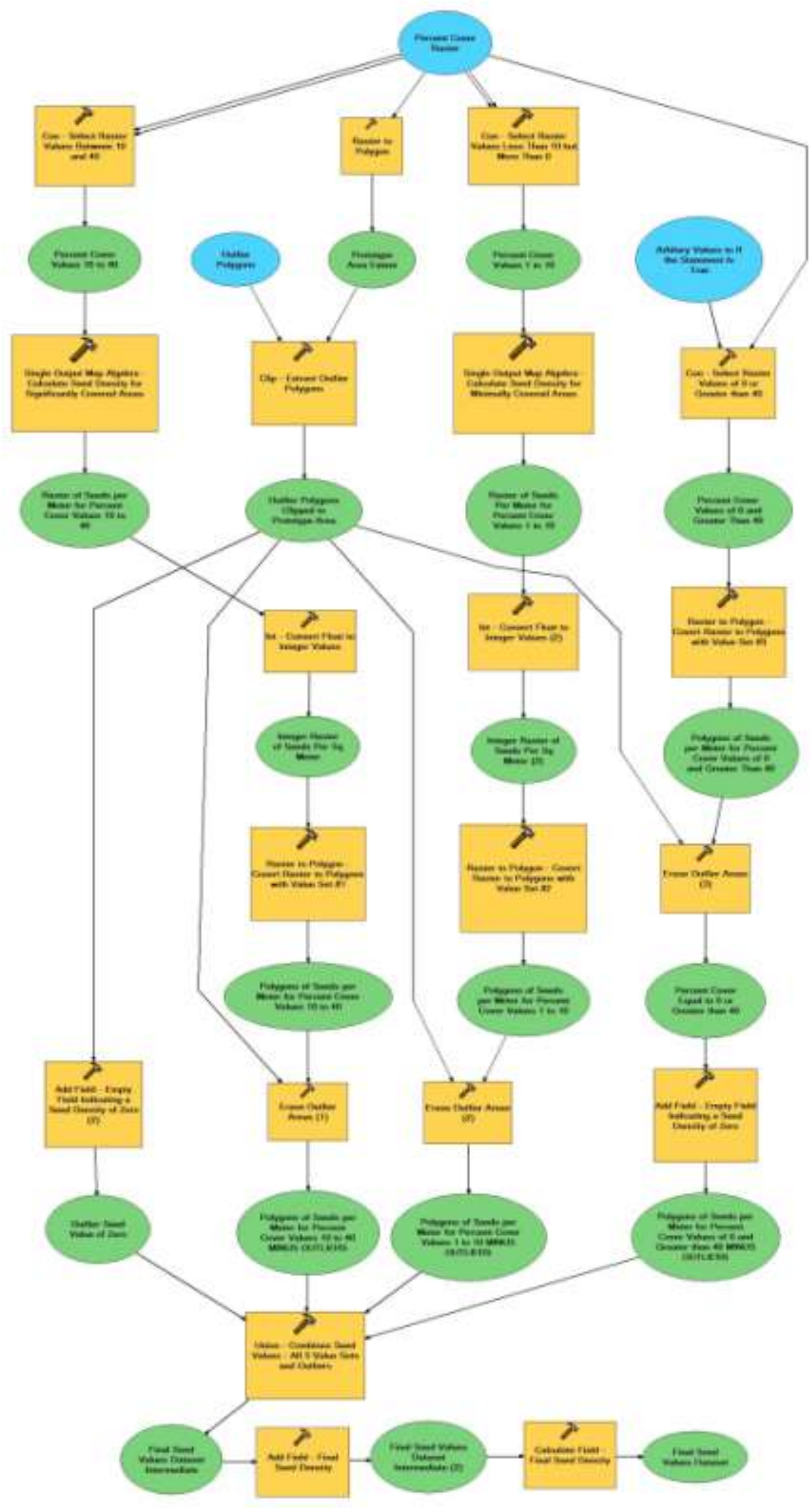





\section{Appendix D - Plants per Meter Calculation Model}

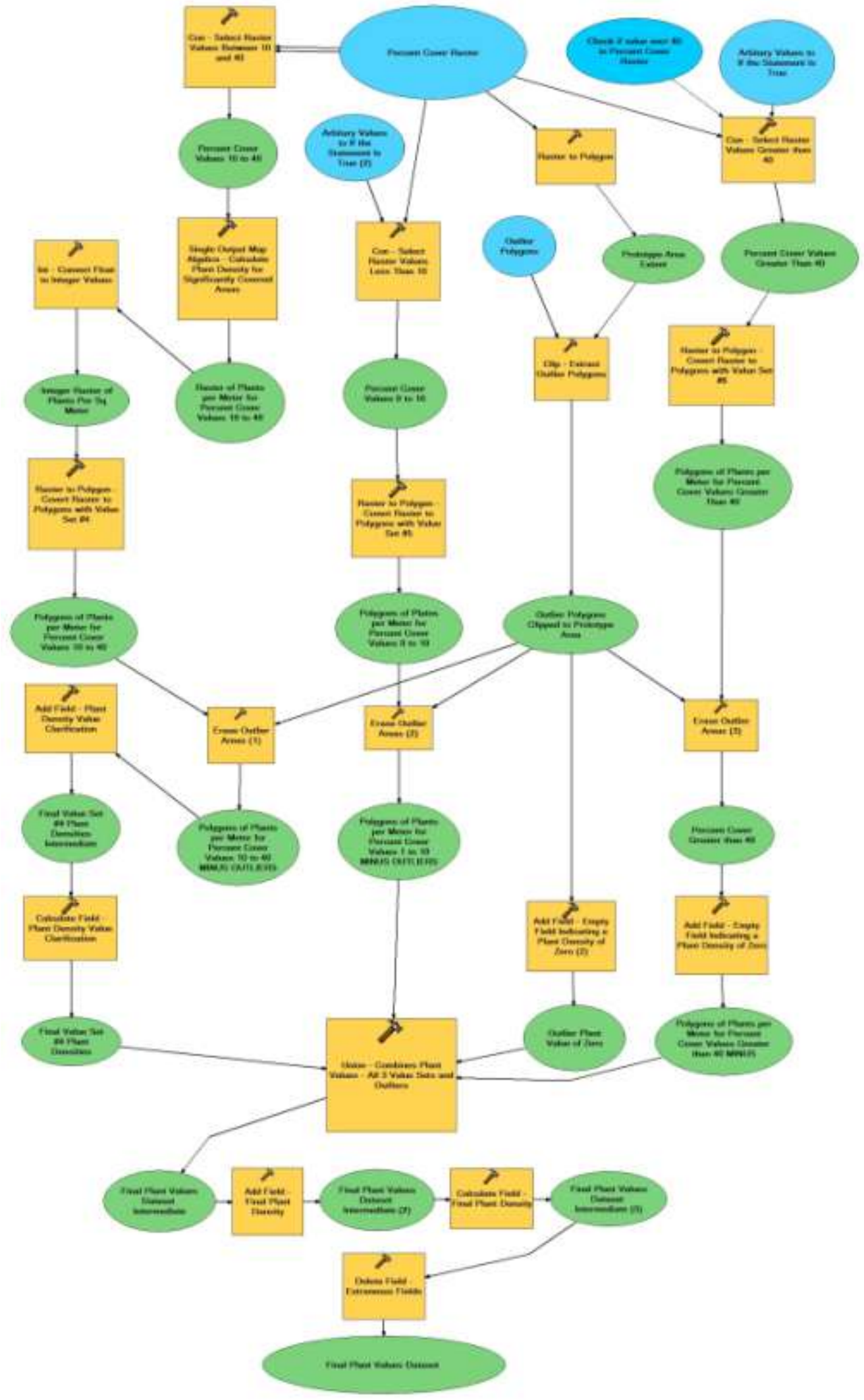





\section{Appendix E - Calculating Number of Agents}

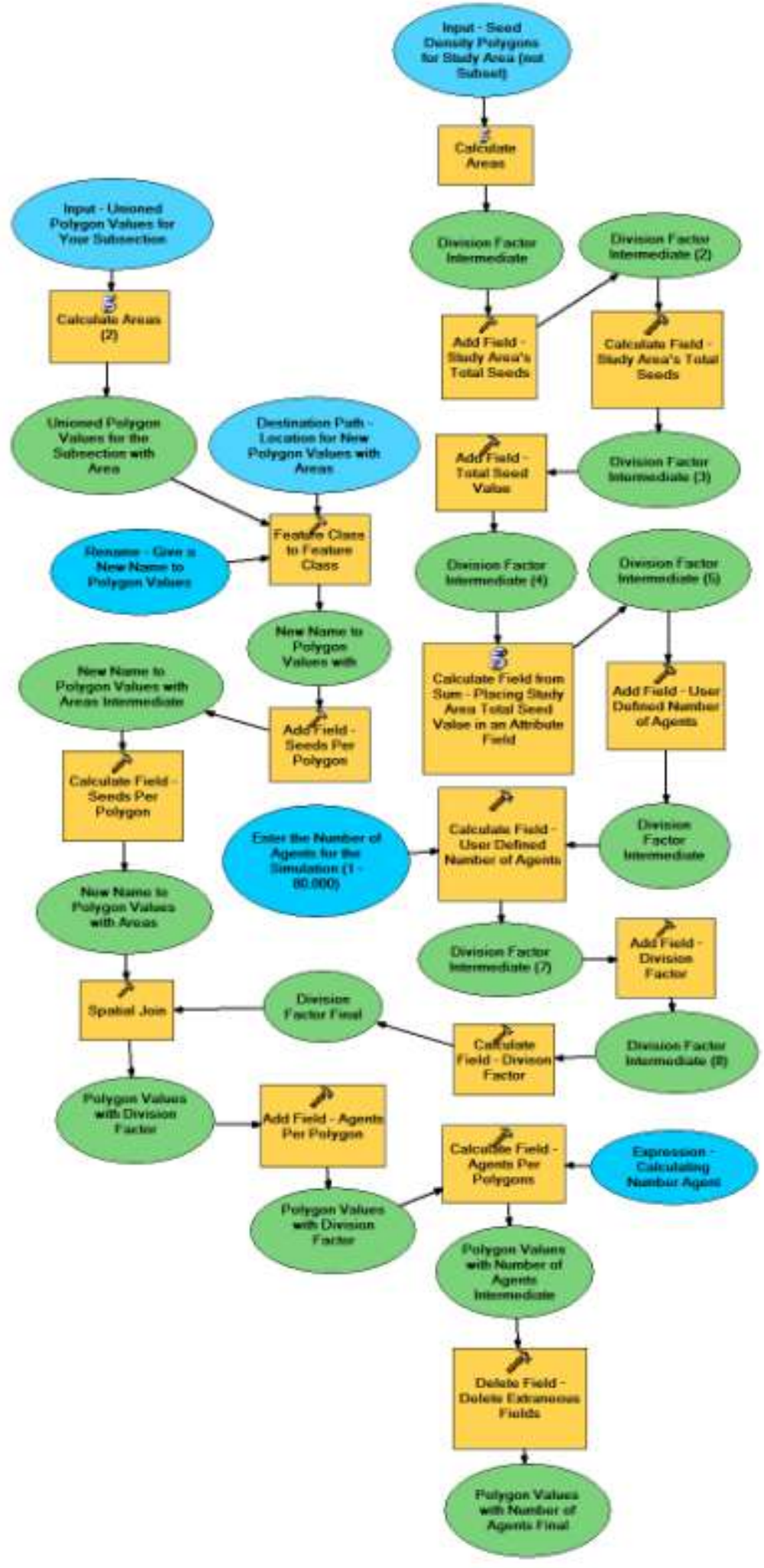





\section{Appendix F - Map User Interface Snap-shot}

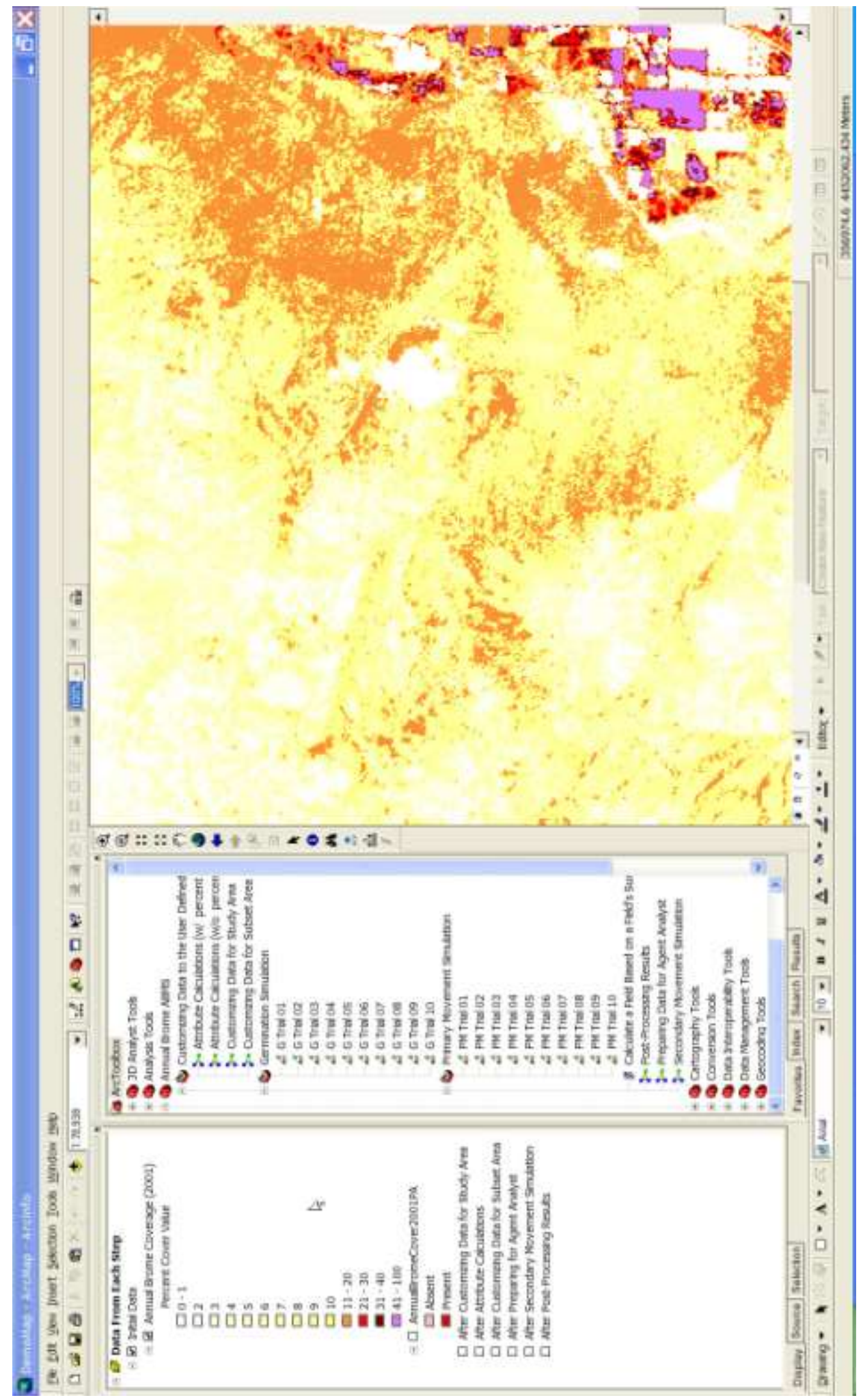





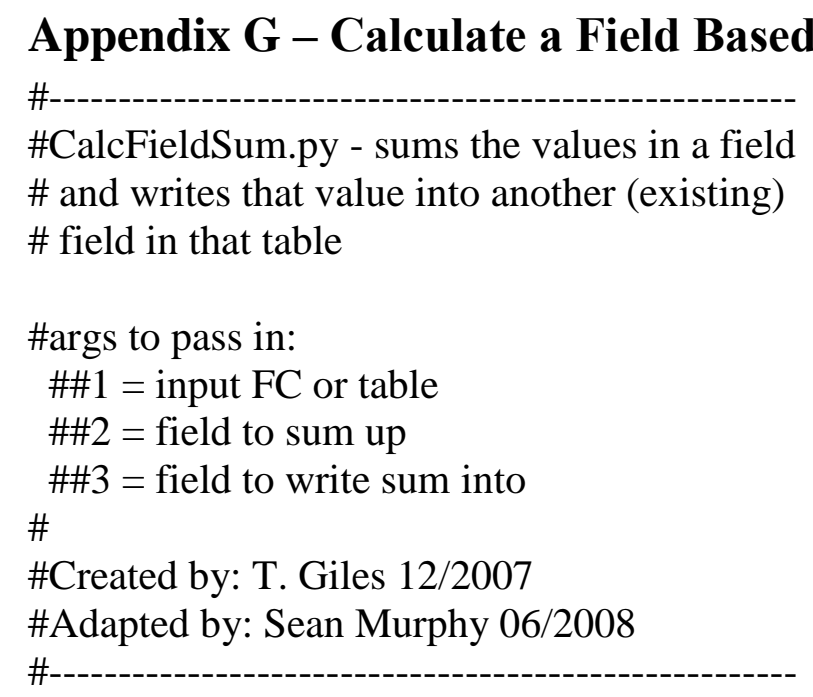

import arcgisscripting, sys

$\mathrm{gp}=$ arcgisscripting.create ()

gp.AddToolbox("C:/Program Files/ArcGIS/ArcToolbox/Toolboxes/Data Management Tools.tbx")

intable $=\operatorname{sys} \cdot \operatorname{argv}[1]$

field $=$ sys.argv[2]

output $=$ sys.argv[3]

\# create search cursor

rows $=$ gp.SearchCursor(intable)

row $=$ rows.Next()

$\mathrm{x}=0.0$

\# Enter while loop for each feature/row

while row:

$\mathrm{x}+=$ row.getvalue(field)

print $\mathrm{x}$

row $=$ rows.next ()

\#note value can be rounded depending on field type

gp.CalculateField_management(intable, output, float(x), "PYTHON") 



\section{Appendix H - Code Variable Definitions}

(In order of appearance; see Appendix J and Appendix M)

Primary Movement Code (Appendix J) -

self.rndl: value in a double precision field defined by the random number generator.

self.rnd2: value in a double precision field defined by the random number generator.

self.Wind: inherited wind value from the data source.

self.TotDenVal: inherited plant density value from the data source.

self.the_geom: the agent position defined by the projection's map units.

self.initialRadius: intermediate travel/spread distance in meters.

self.finalRadius: final travel/spread distance in meters.

self.rnd3: value in a double precision field defined by the random number generator.

self.sprdDirD: travel azimuth/direction in degrees (due east is zero).

self.sprdDirA: travel azimuth/direction in radians (due east is zero/ $2 \pi$ ).

self.addX: travel distance the agent moved along the $\mathrm{X}$-axis.

self.addY: travel distance the agent moved along the $\mathrm{Y}$-axis.

self.the_geom.coordinate: the agent position in $\mathrm{X}$ and $\mathrm{Y}$ coordinates defined by the projection's map units.

coordinate.x: original X-coordinate for an agent's position.

coordinate.y: original Y-coordinate for an agent's position.

Germination Code (Appendix M) -

self.the_geom.coordinate: the agent position in $\mathrm{X}$ and $\mathrm{Y}$ coordinates defined by the projection's map units.

self.rndl: value in a double precision field by the random number generator.

self.Prob: inherited habitat probability value from the data source.

coordinate.x: original $\mathrm{X}$-coordinate for an agent's position.

coordinate.y: original Y-coordinate for an agent's position. 



\section{Appendix I - Primary Movement Code}

def step():

\# Find a random number between 0 and 1

\# and then if it is greater than the wind

\# value, then the seed does not move.

\# However, if it is less than the wind

\# value, then the seed moves depending on

\# the variables in the else statement.

self.rnd1 $=$ Random.uniform.nextDouble $($ )

self.rnd2 $=$ Random.uniform.nextDouble()

if self.rnd $1>$ self.Wind or self.rnd $2>$ self.TotDenVal:

self.the_geom $=$ self.the_geom

\# The initial radius and the final radius variables

\# are a result of a logistic regression equation

\# calculated from scientific literature. The wind

\# and plant density value determine the distance

\# an agent travels.

else:

self.initialRadius $=($ self.Wind $*$ self.TotDenVal -0.4861$) /-0.175$

self.finalRadius $=1$ / Math.exp(self.initialRadius)

\# Find a random number between 0 and 1

\# and then multiple that by the maximum

\# degree direction (359.9- degrees). This

\# results in a random direction.

self.rnd3 = Random.uniform.nextDouble $($ )

self.sprdDirD = 359.99999999999999* self.rnd3 


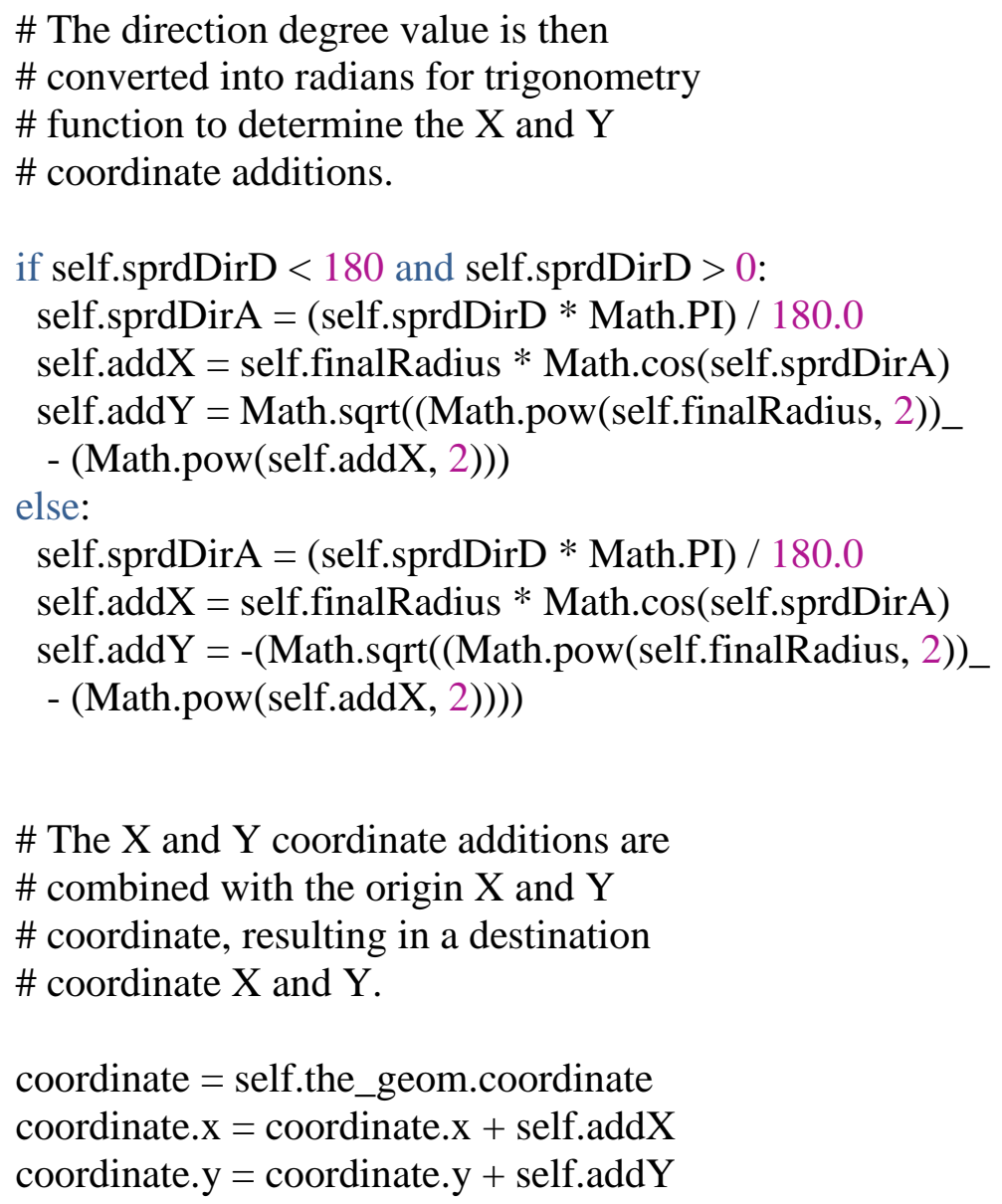


Appendix J - Concept for Programming Change of X and Y

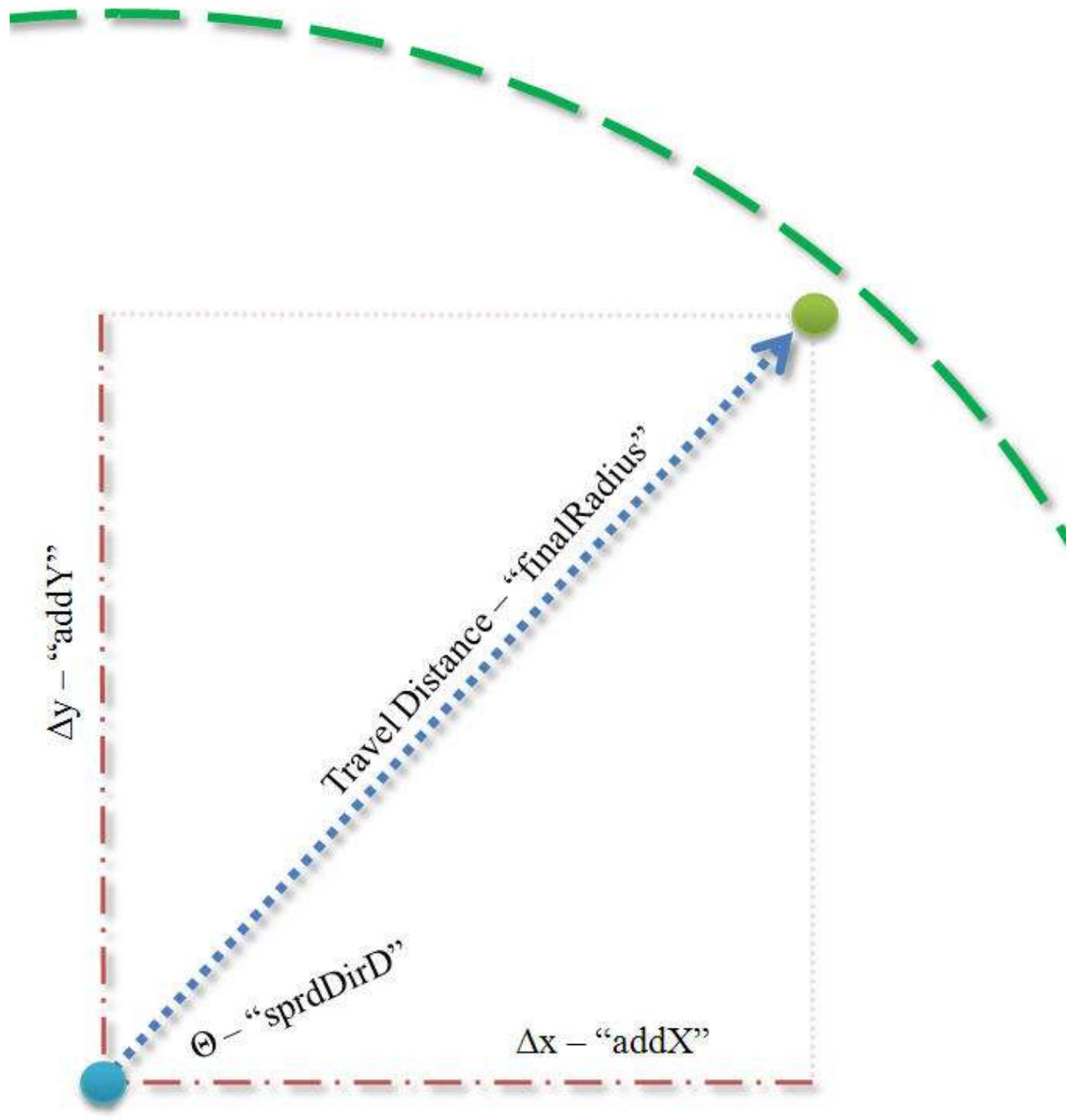





\section{Appendix K - Separation of Road and Shoulder Model}

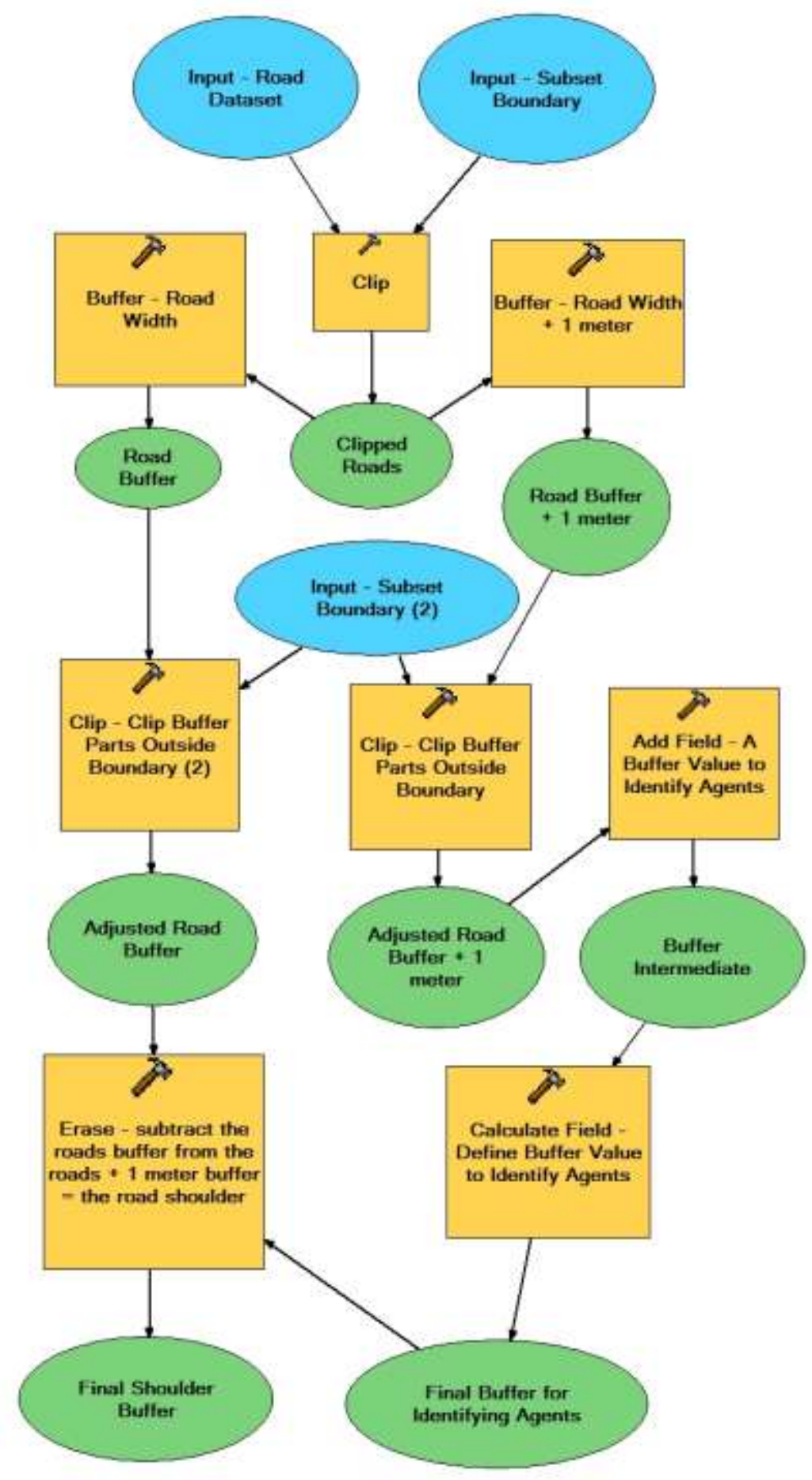





\section{Appendix L - Germination Simulation Code}

def step():

coordinate $=$ self.the_geom.coordinate

self.rnd $1=$ Random.uniform.nextDouble()

if self.rnd1 > self.Prob:

coordinate $\mathrm{x}=$ coordinate $\mathrm{x}$ - coordinate $\mathrm{x}$

coordinate. $y=$ coordinate.$y$ - coordinate. $y$ 



\section{Appendix M - Repolygonizing Agents Model}

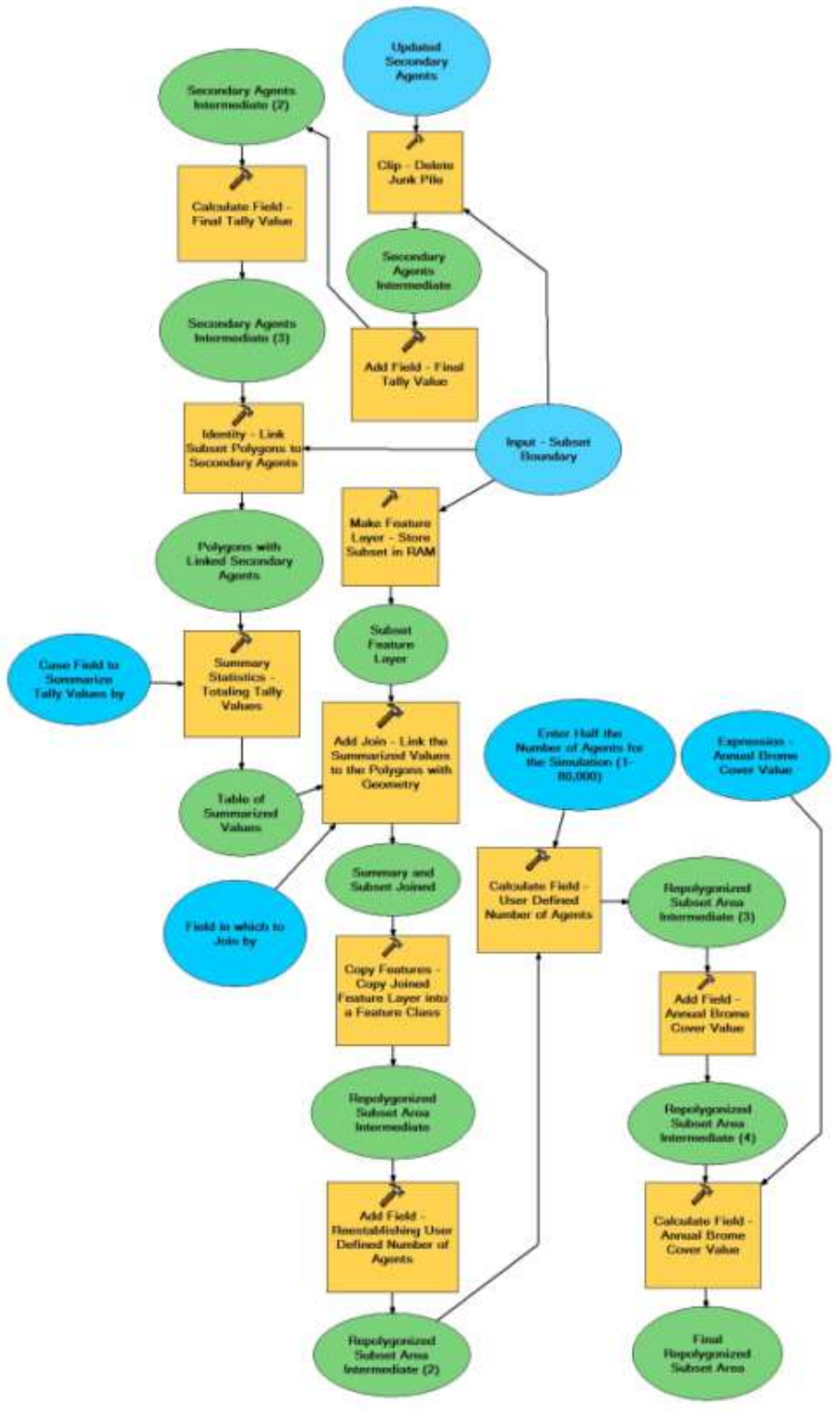





\section{Appendix N - Reclassification Model}

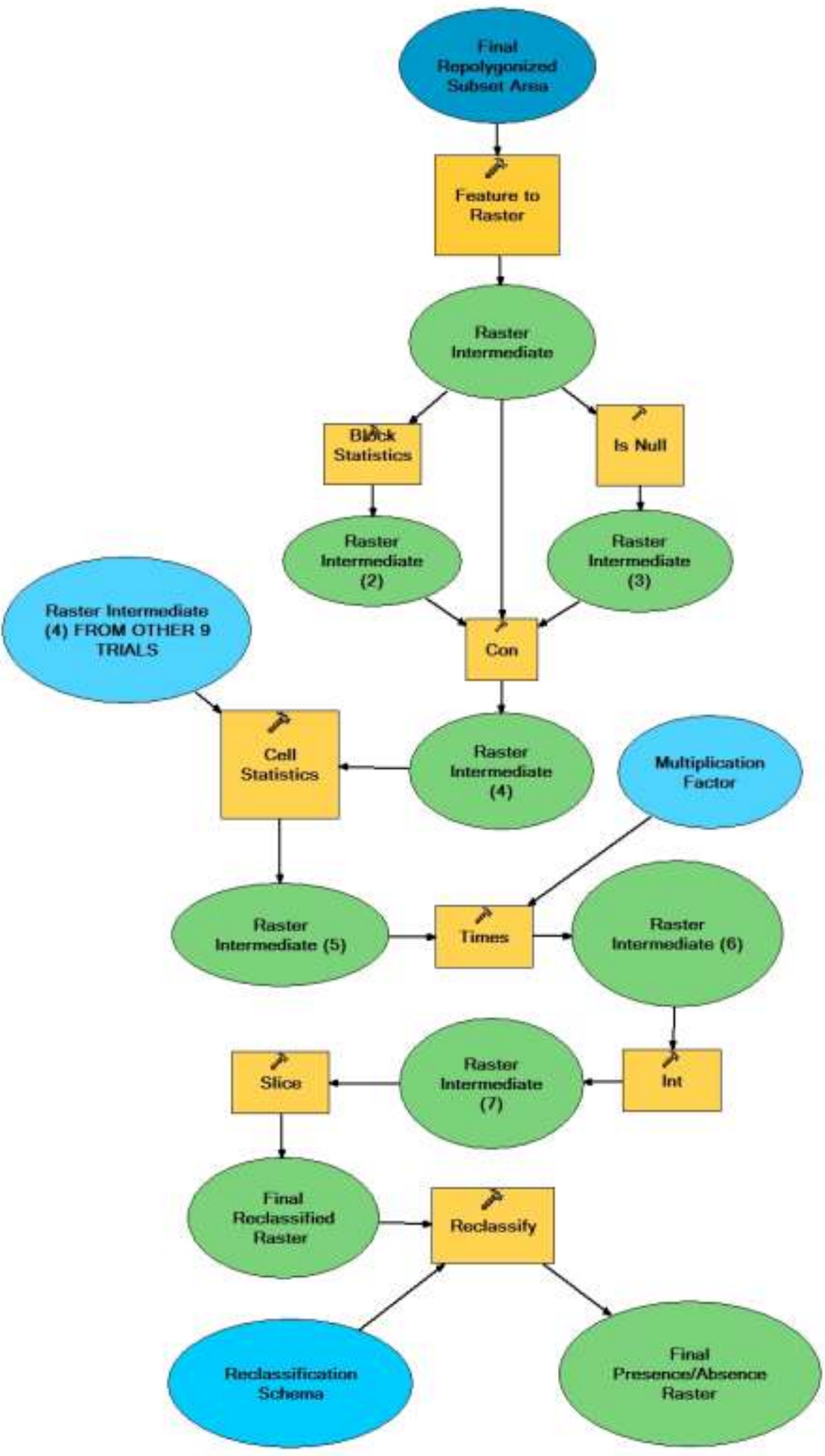





\section{Appendix O - Web Application Information and Snap-shots}

Web Application URL: http://msgis-11/Annual\%20Brome\%20ABMS/default.aspx

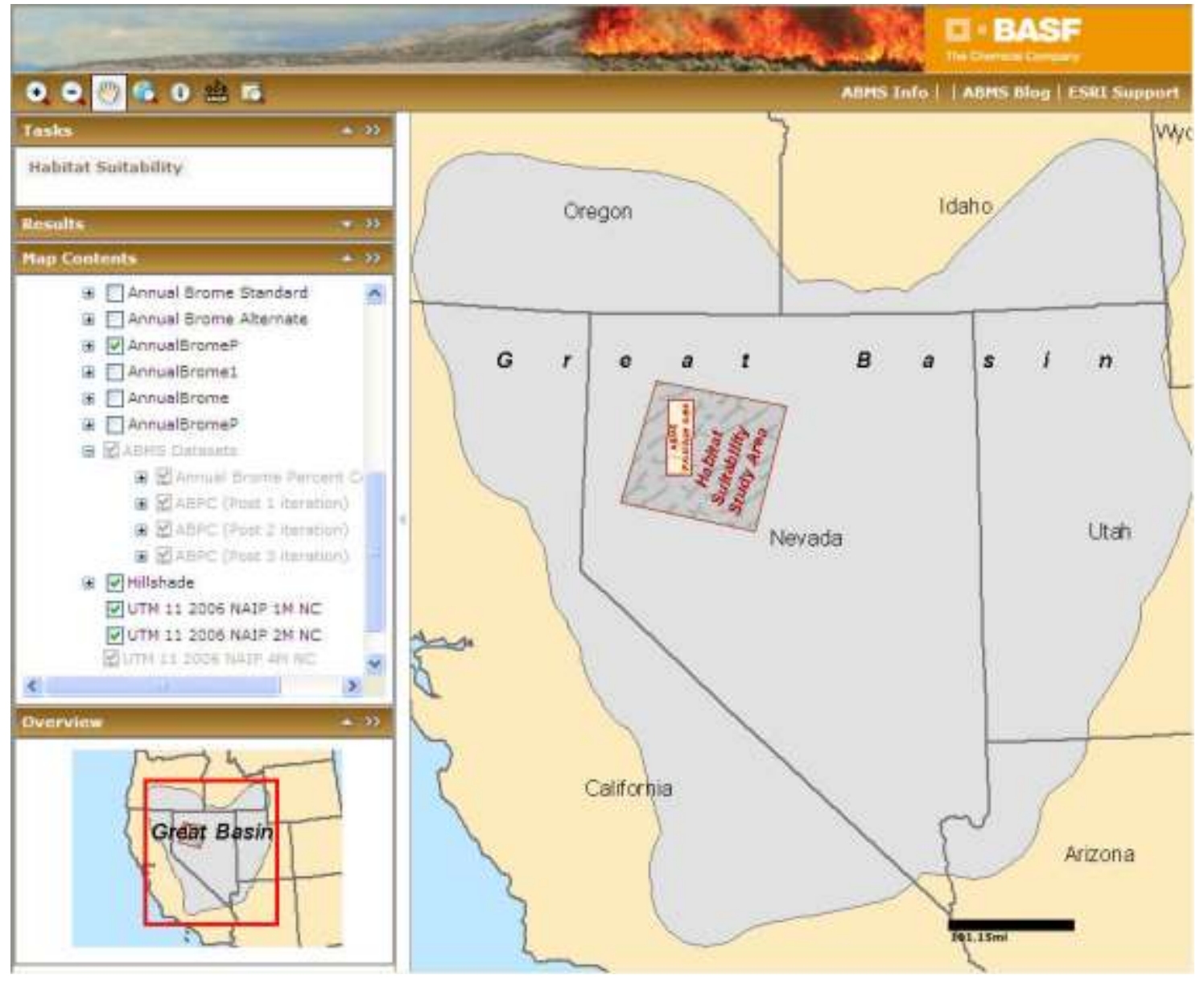

The default view of the web application. 


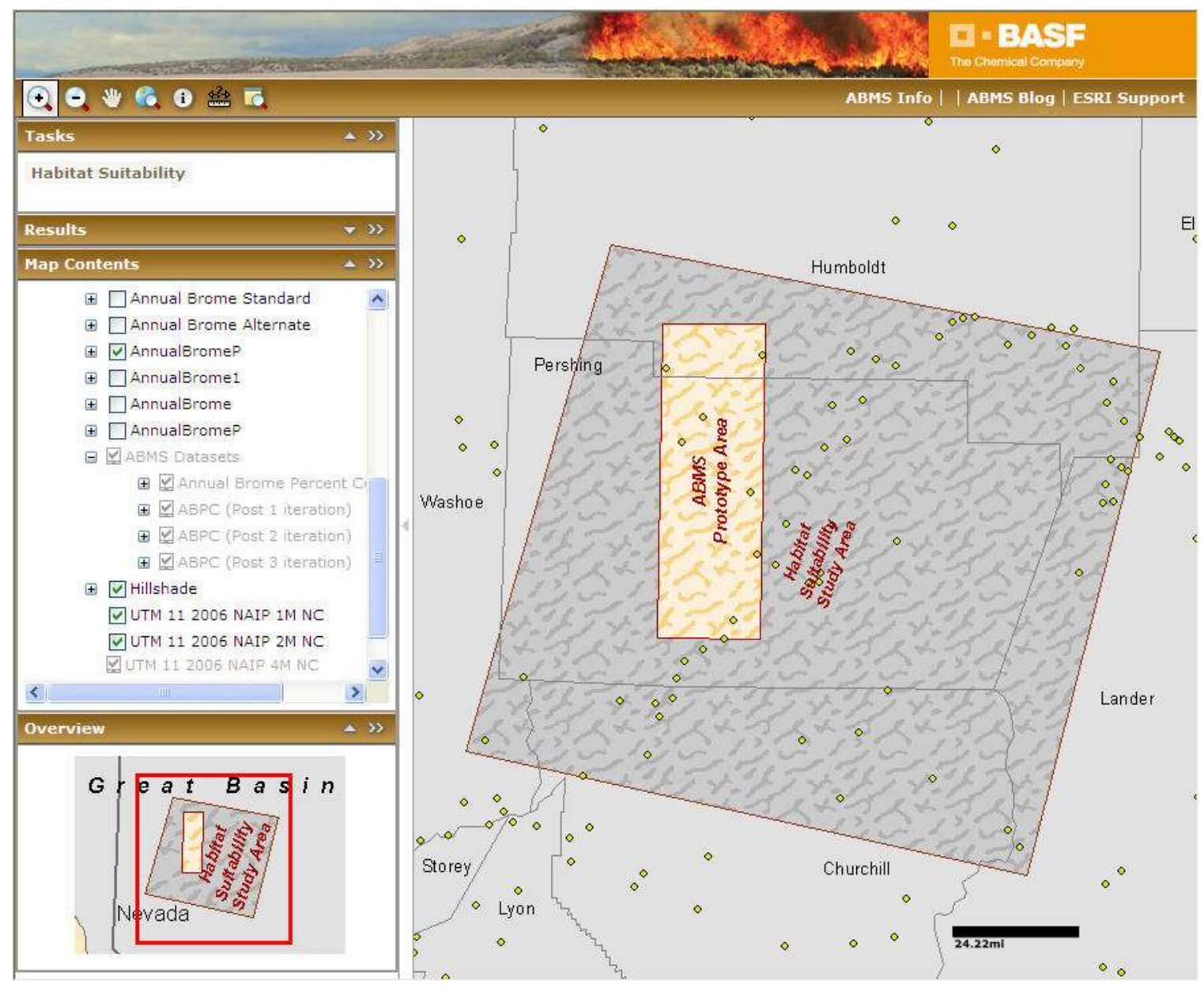

Zoomed to Armstrong's study area. 


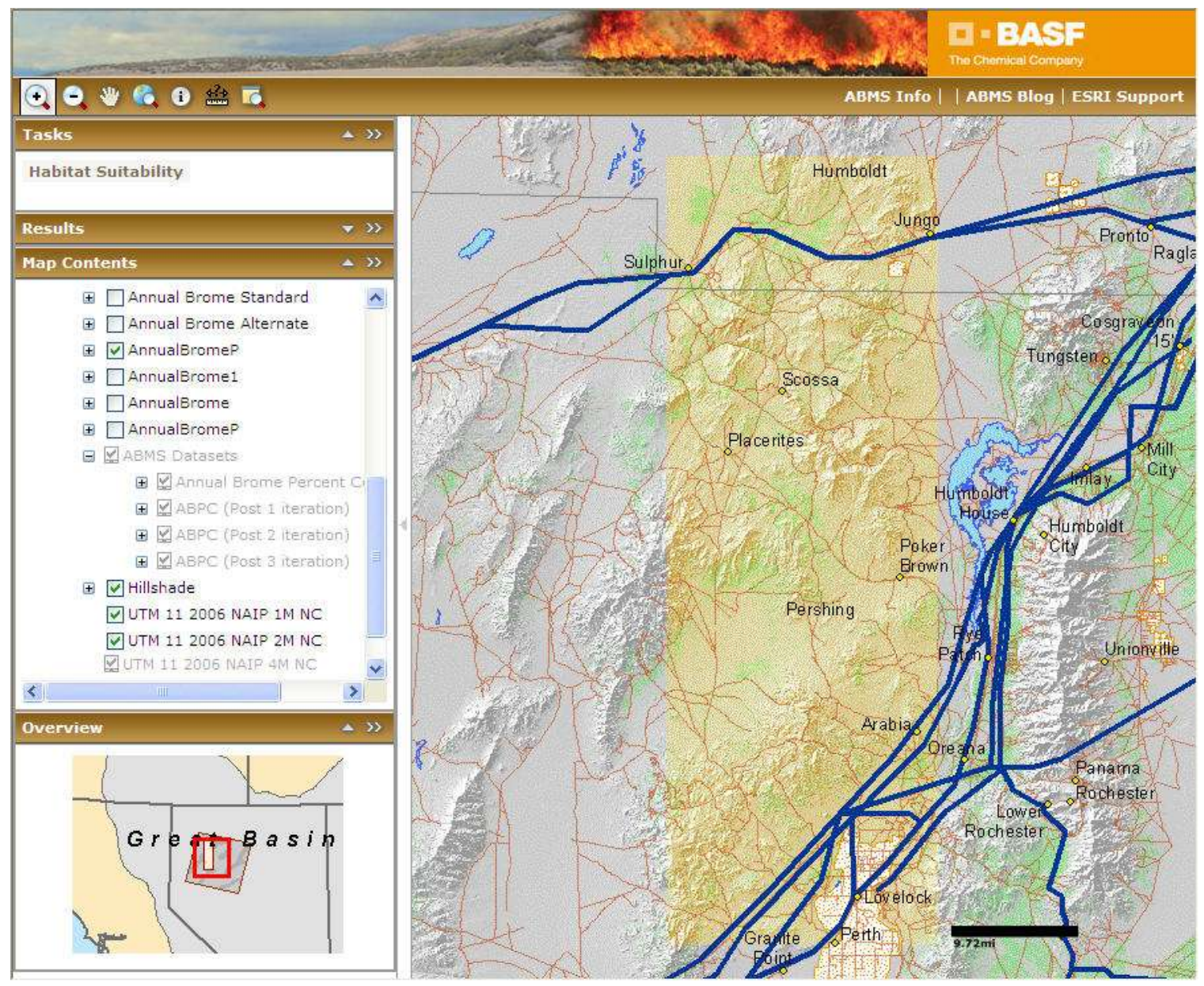

Zoomed to the prototype area with a hillshade background. 


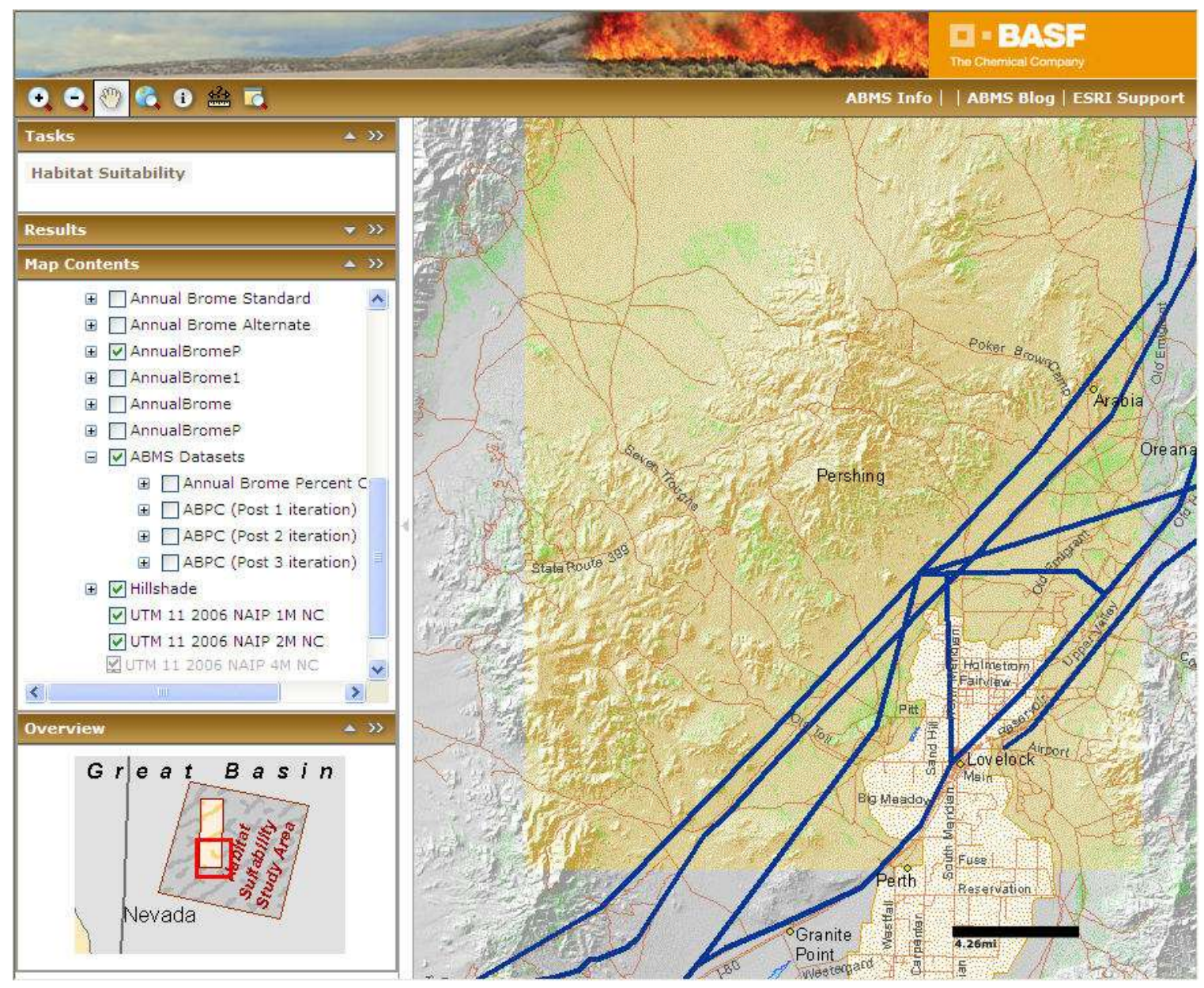

Zoomed to Lovelock and its northern landscape. 


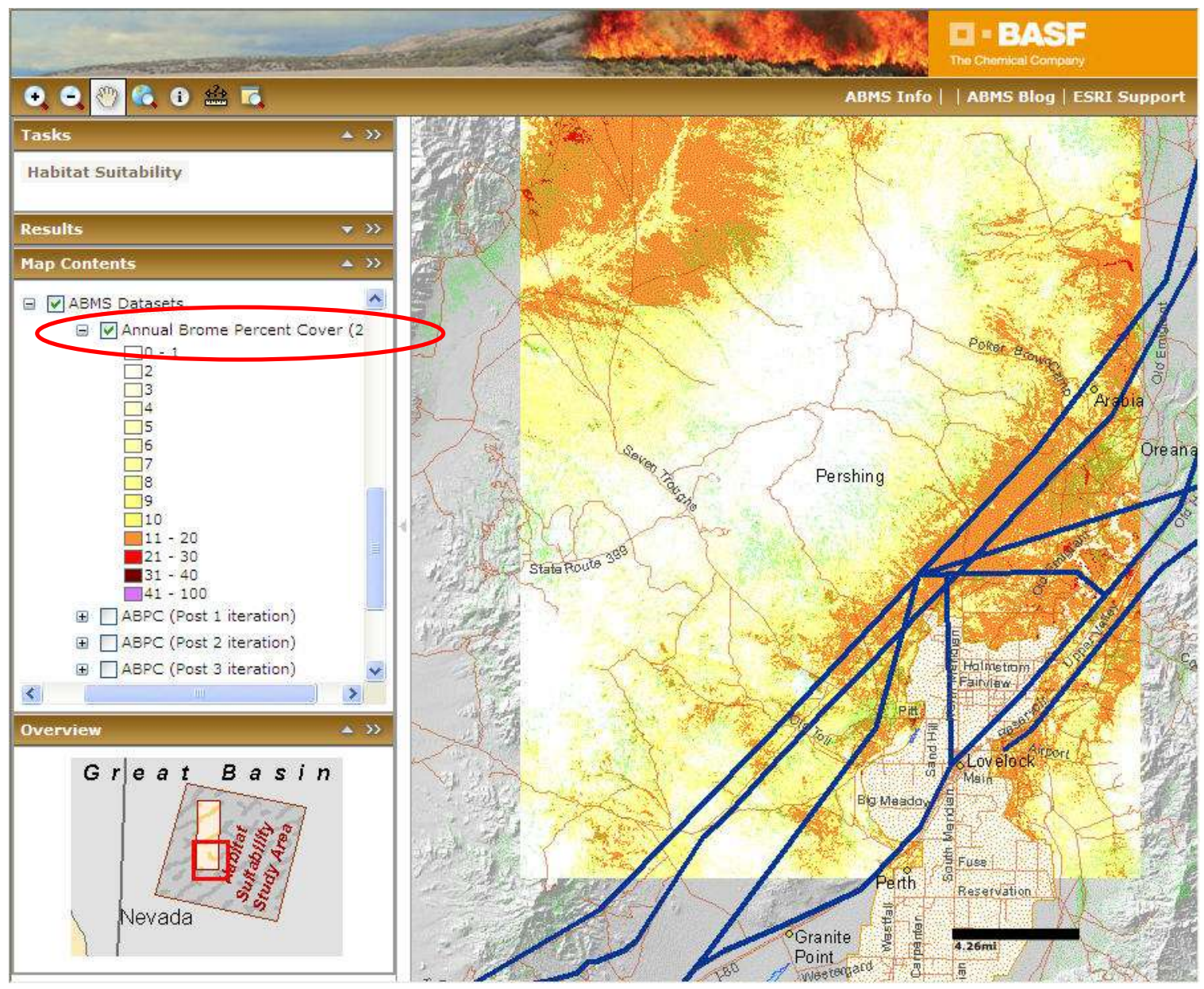

The web application displaying Lovelock with the 2001 annual brome percent cover symbolized. 


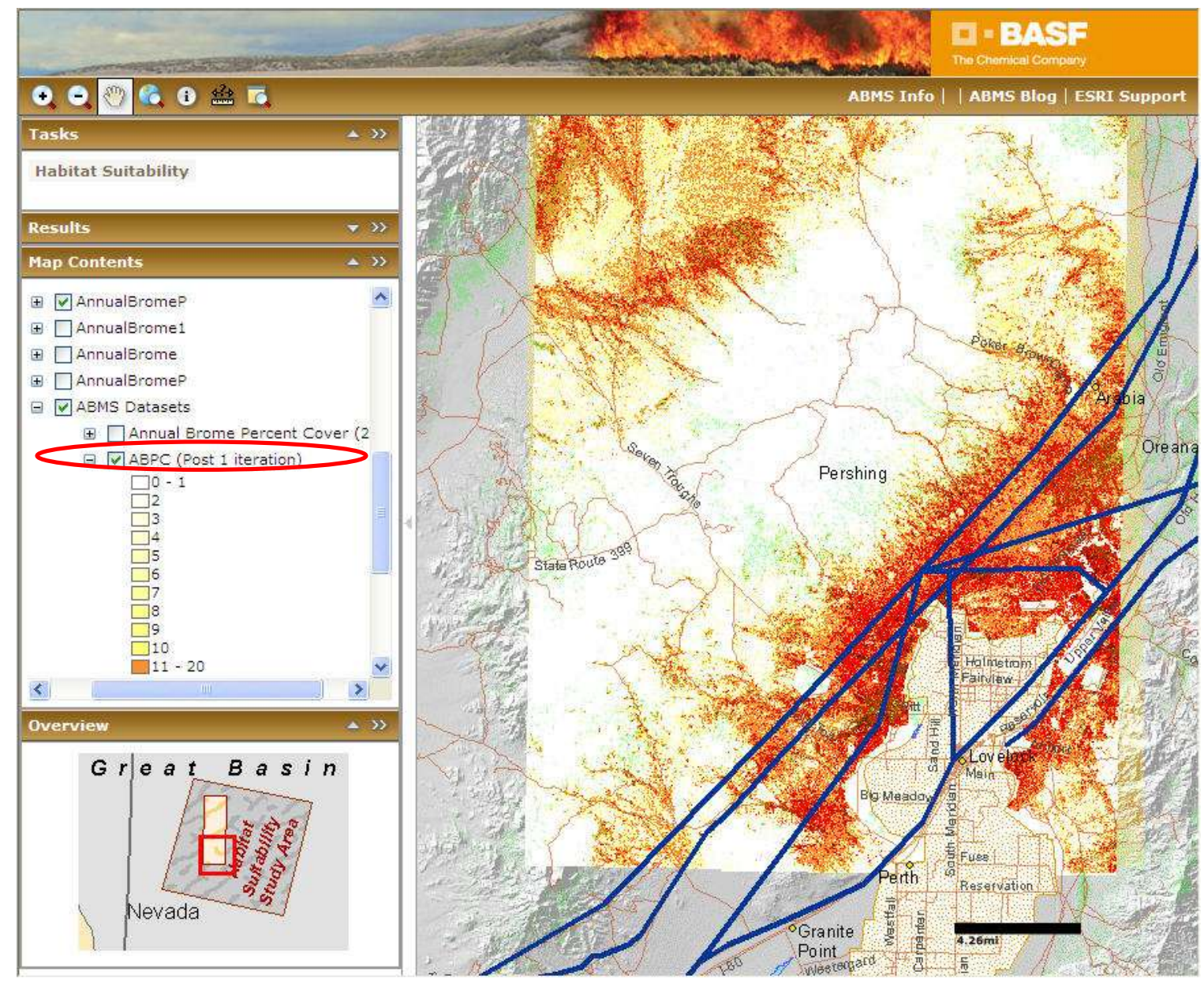

The web application displaying Lovelock after the first run of the agent-based model. 


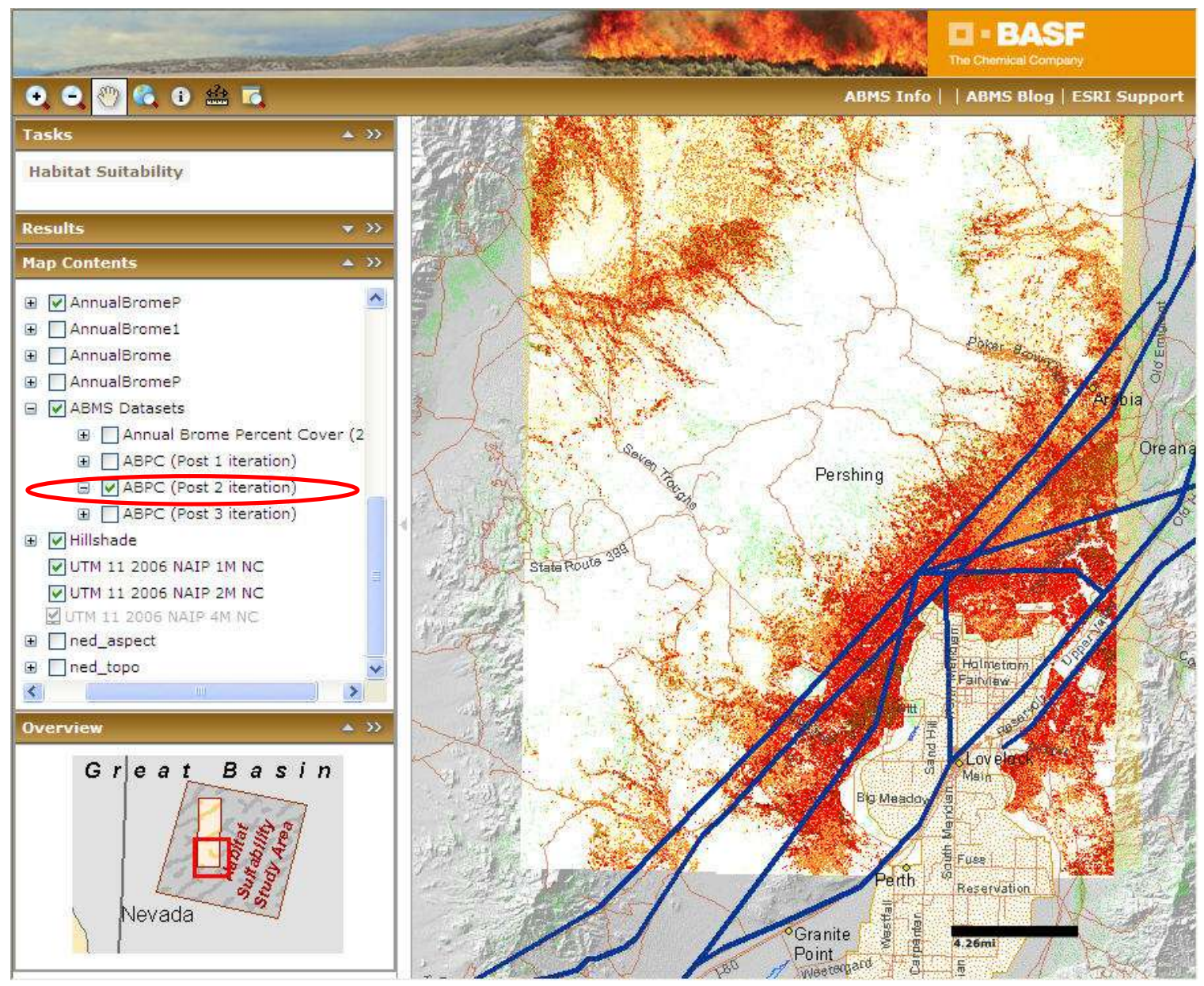

The web application displaying Lovelock after the second run of the agent-based model. 


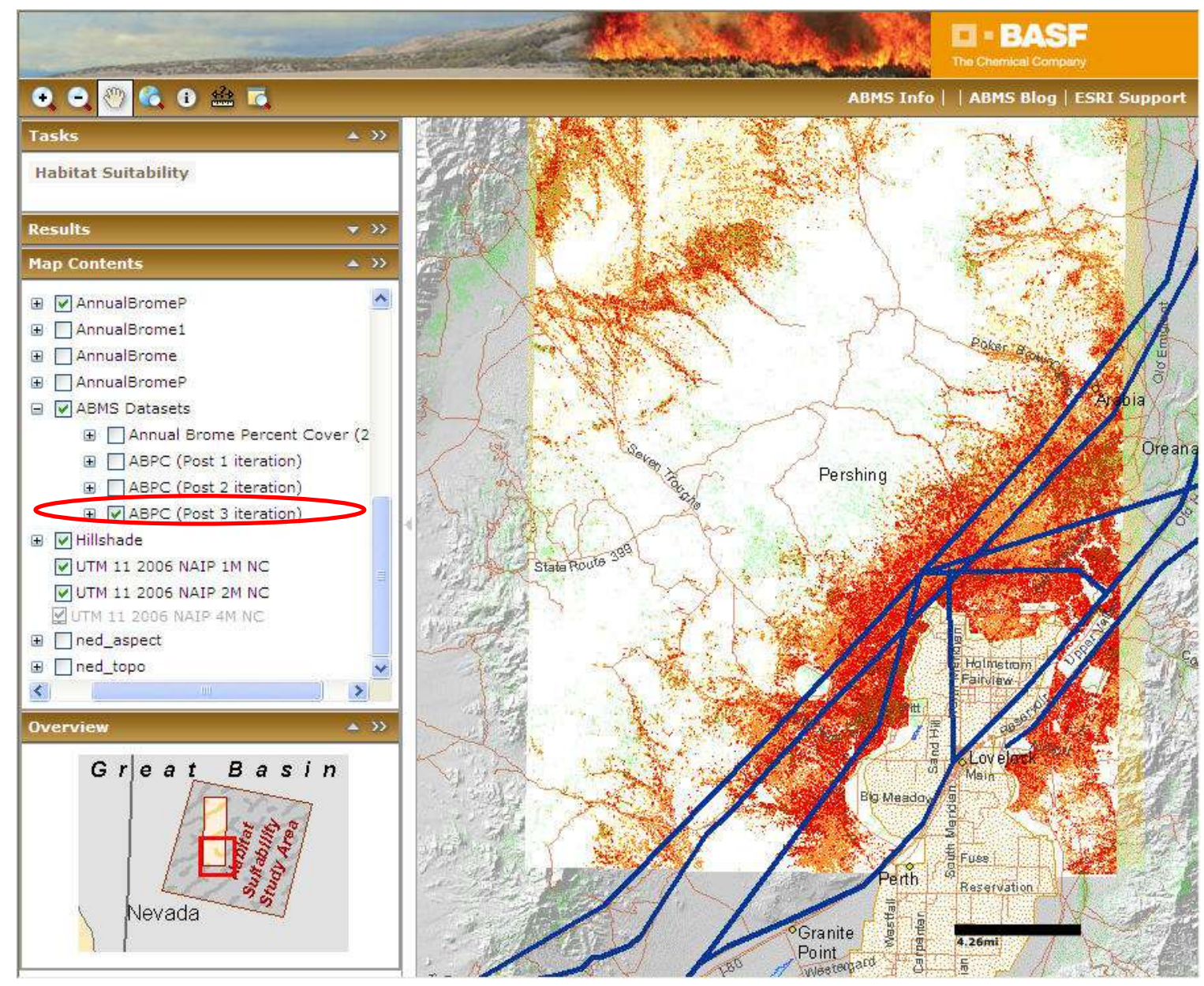

The web application displaying Lovelock after the third run of the agent-based model. 


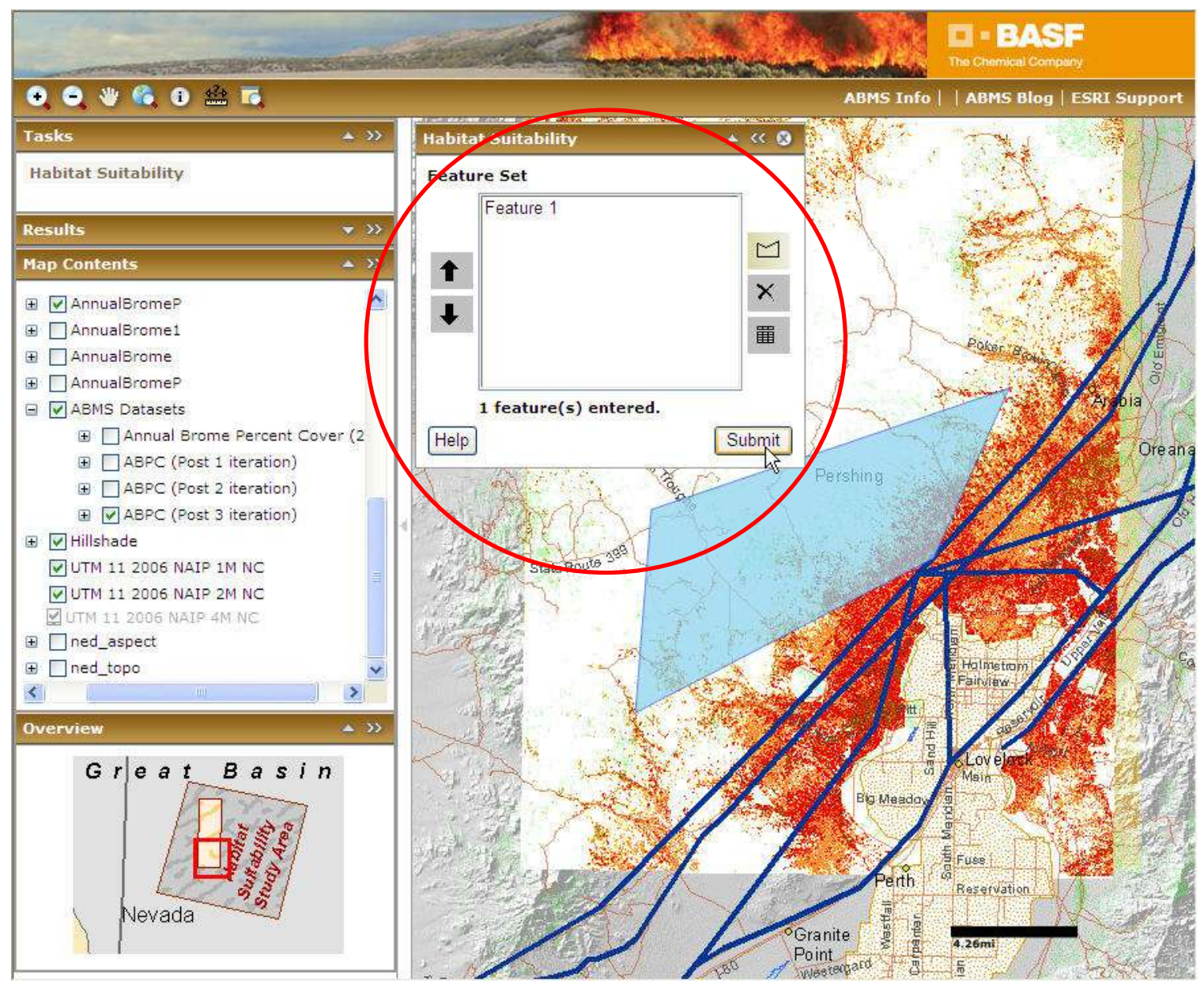

The process of implementing Armstrong's live geoprocessing habitat suitability model with the agent-based modeling results. 